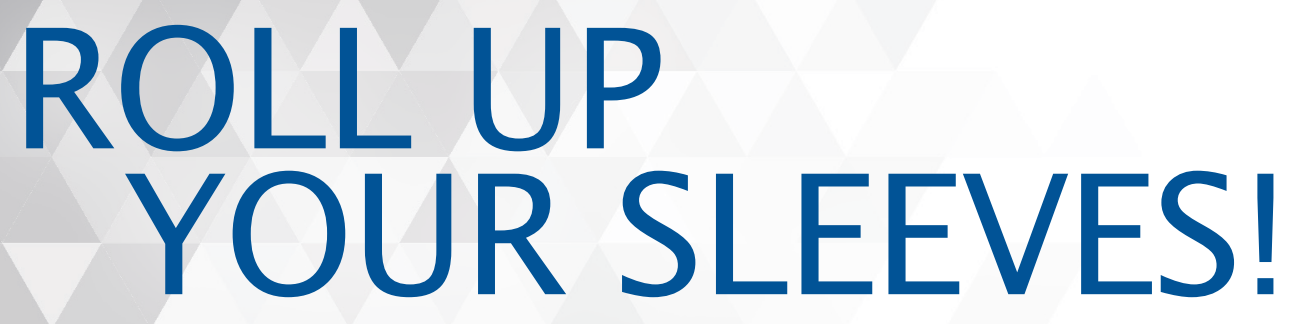

\title{
TECHNOLOGY-SUPPORTED ARM AND HAND TRAINING AT HOME AFTER STROKE
}

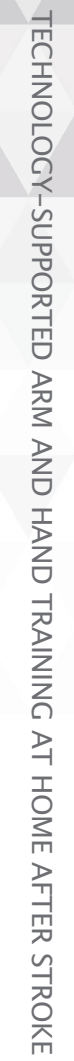






\section{ROLL UP YOUR SLEEVES!}

\section{TECHNOLOGY-SUPPORTED ARM AND HAND TRAINING AT HOME AFTER STROKE}

Sharon Nijenhuis 
Part of the work in this thesis was performed within the SCRIPT (Supervised Care \& Rehabilitation Involving Personal Telerobotics) project, partly funded by the European Commission Seventh Framework Program under grant no. FP7-ICT-288698.

The publication of this thesis was generously supported by:

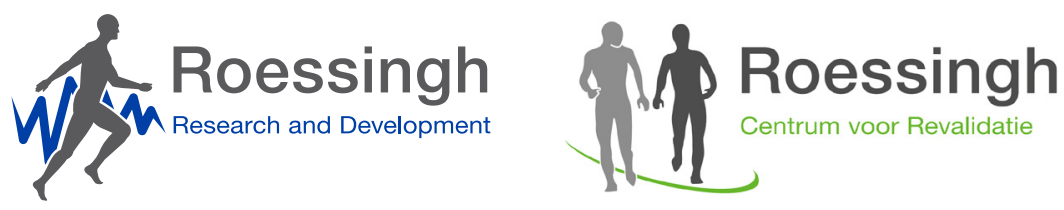

Cover design: Stefan te Loeke - loek \& feel

Printed by: Gildeprint - The Netherlands

ISBN: 978-90-365-4510-5

DOI: $10.3990 / 1.9789036545105$

(C) Sharon Nijenhuis, Enschede, the Netherlands, 2018

All rights reserved. No part of this book may be reproduced, stored in a retrieval system, or transmitted, in any form or by any means, electronic, mechanical, photocopying, recording, or otherwise, without prior written permission of the holder of the copyright. 


\section{ROLL UP YOUR SLEEVES!}

\section{TECHNOLOGY-SUPPORTED ARM AND HAND TRAINING AT HOME AFTER STROKE}

PROEFSCHRIFT

ter verkrijging van

de graad van doctor aan de Universiteit Twente, op gezag van de rector magnificus, prof. dr. T.T.M. Palstra volgens besluit van het College voor Promoties

in het openbaar te verdedigen

op vrijdag 20 April 2018 om 16.45 uur

door

Sharon Maria Nijenhuis

geboren op 25 oktober 1986

te Lichtenvoorde 
Dit proefschrift is goedgekeurd door

Prof. dr. J.S. Rietman (promotor)

Prof. dr. J.H. Buurke (promotor)

Dr. G.B. Prange-Lasonder (co-promotor)

(C) Sharon Nijenhuis, Enschede, the Netherlands, 2018

ISBN: 978-90-365-4510-5 


\section{Promotiecommissie}

Voorzitter / secretaris

Prof. dr. G.P.M.R. Dewulf

Universiteit Twente

\section{Promotoren}

Prof. dr. J.S. Rietman

Universiteit Twente

Prof. dr. J.H. Buurke

Universiteit Twente

\section{Co-promotor}

Dr. G.B. Prange-Lasonder

Universiteit Twente

\section{Leden}

Prof. dr. ir. H. van der Kooij

Universiteit Twente

Prof. dr. ir. H.J. Hermens

Universiteit Twente

Prof. dr. G.M. Ribbers

Erasmus Universiteit Rotterdam

Prof. dr. C.K. van der Sluis

Rijksuniversiteit Groningen

Dr. F. Amirabdollahian

University of Hertfordshire 



\section{TABLE OF CONTENTS}

Chapter 1 General introduction 9

Chapter 2 Training modalities in robot-mediated upper limb rehabilitation in stroke: a framework for classification based on a systematic review

Chapter 3 Direct effect of a dynamic wrist and hand orthosis on reach and grasp kinematics in chronic stroke

Chapter 4 Feasibility study into self-administered training at home using an arm 75 and hand device with motivational gaming environment in chronic stroke

Chapter 5 Effects of training with a passive hand orthosis and games at home in chronic stroke: a pilot randomised controlled trial

Chapter 6 Strong relations of elbow excursion and grip strength with post stroke arm function and activities: should we aim for this in technologysupported training?

Chapter 7 Feasibility of a second iteration wrist and hand supported training system for self-administered training at home in chronic stroke

Chapter 8 General discussion

$\&$ Summary 166

Samenvatting 169

Dankwoord 172

About the author

175

Progress range 



\section{Chapter 1}

General introduction 


\section{STROKE}

\section{"I would like to drive a car again, improve my sloppy handwriting, and just pick up a cup of coffee."}

This quote describes some activities in daily life which are common for healthy people. However, this is a goal from one of the patients with impaired arm and hand function following stroke who participated in one of my studies, and for whom this is not normal anymore. Each year about 15 million people worldwide experience a new or recurrent stroke, resulting in 5 million deaths each year. ${ }^{1}$ Many of the remaining stroke survivors live with permanent disability. Due to the aging population, the prevalence of stroke is likely to increase even more in future, consequently increasing the burden on healthcare professionals. Stroke can result in a variety of symptoms, including sensory, cognitive, psychological and motor problems. For many stroke patients motor problems are a major issue, for example resulting in muscle weakness, spasticity or disturbed coordination. ${ }^{2}$ This impaired arm and hand function may result in serious limitations in the performance of activities of daily living, resulting in being more dependent on other people. This can affect patients immediately after stroke. The long-term effects of stroke are based on the location and size of the stroke lesion, and the amount of recovery. This recovery is a complex process that involves a combination of spontaneous recovery and learning-dependent processes. . $^{3,4}$

In the first few days after the event, processes of spontaneous neurological recovery can occur. Some of these mechanisms involve restitution of non-infarcted penumbral areas, reduction of edema around the lesion, and resolution of diaschisis, in which remote cortical tissue is temporarily suppressed after focal cortical injury. ${ }^{3,5,6}$ A longer term mechanism involves neural plasticity, which means that changes in cortical representation occur during recovery. Other cortical structures, either adjacent to or remote from the damaged area can 'take over' the function of the damaged area. This vicariation of function involves processes of unmasking of previously present but functionally inactive connections, and axonal sprouting in which undamaged axons grow new nerve endings to reconnect injured neurons..$^{3,5} 6$ In order to reconnect, the neurons need to be stimulated through activity. In addition, recovery occurs in a large part through behavioral compensation, such as trunk compensation to accomplish reaching. ${ }^{4}$ Permitting the use of motor compensations could in the long term lead to a pattern of learned nonuse of the affected upper extremity. ${ }^{7}$ The stimulation of the use of the affected upper extremity has shown to overcome the learned nonuse. ${ }^{8}$ Such learning-dependent processes can be influenced by post-stroke rehabilitation. This requires an immense perseverance of stroke patients, they need to 'roll up their sleeves' and start and maintain rehabilitation training. 


\section{STROKE REHABILITATION}

Since many stroke patients suffer from limitations in the performance of activities of daily living, one of the major goals is rehabilitation of impaired movements and the associated functions. Many stroke rehabilitation interventions are currently available, all describing a different training approach (such as in interventions related to gait or arm-hand activities, interventions for activities of daily living, or interventions for physical fitness), or targeting a specific group of patients (such as phase post-stroke). ${ }^{9-12}$ In stroke rehabilitation it is important to involve aspects related to the underlying mechanisms of motor recovery. Rehabilitation protocols are being based on a neurophysiological basis for key aspects that stimulate restoration of arm function. Functional task-specific training, high intensity training and frequent movement repetitions are well accepted principles of motor learning. Additionally, training should preferably be provided in the patient's own (motivational) environment with active contribution of the patient. ${ }^{2,10,12-14}$

\section{TECHNOLOGY-SUPPORTED TRAINING DEVICES}

High intensity training with a large number of repetitions can be facilitated by technologysupported upper extremity training, such as robot-assisted training. ${ }^{15-17}$ Besides, technologysupported training has the advantage that it does not require direct supervision from a therapist. This will relieve the burden on healthcare professionals by no longer offering direct support and saving one-on-one treatment time. Many different types of technologysupported devices are available, differing for example in mechanical design (e.g. endeffector or exoskeleton), control strategy (type of assistance) or the joints they target (e.g. shoulder-elbow, wrist-hand or shoulder-elbow-wrist-hand). ${ }^{17}$ The modalities of training these devices provide are related to conventional therapy modalities important for improvement of upper extremity motor control and function. One of these modalities is passive movement, in which a patient's effort is not required but completely taken over by the device. Other modalities are for example active movement that is partially assisted by the device, or resistive training in which the device provides force opposing the movement. Many robotic devices can involve a mixture of training modalities, but the best therapy strategy is not clear yet, although active contribution of the patient is desirable. Several studies have shown (moderate) positive effects of robotic upper extremity therapy after stroke. ${ }^{15,18-20}$ However, effects are specific to the joints targeted and no generalization is found to improvements in upper limb capacity. ${ }^{15}$ In addition, the added value of robotic therapy over dose-matched conventional therapy is arguable. ${ }^{16,17,19,20}$ This underlines the need for the development of more advanced technology-supported devices focusing on training of functional movements. 
Many of the early developed robotic devices were designed for the proximal upper extremity, allowing movement training for the shoulder and elbow. However, these studies did not show a transfer of motor gains to improvements in performance of activities of daily living, probably due to targeting only the proximal arm. ${ }^{20}$ Devices involving integration of proximal with distal arm training are favorable to enhance functional gains. ${ }^{13,}{ }^{21} \mathrm{New}$ technology-supported devices and advancements of existing devices are continuously being designed. More insight is needed into the best therapy strategy and adequate design of robotic upper extremity devices. ${ }^{15,17}$ Preferably this would involve active contribution of the patient performing functional movements of the proximal and distal arm integrated, and ways to provoke a high intensity of training, for example by means of a motivational environment and meaningful feedback.

\section{INDEPENDENT REHABILITATION AT HOME}

To extend training opportunities and consequently enhance training dose, a next step would be to provide technology-supported systems at home for independent practice. Only few studies performing independent, technology-supported training at home after stroke have been reported yet, and the evidence for remote rehabilitation interventions after stroke is inconclusive yet. ${ }^{22-27}$ Many of these studies describe remote interventions, but still with direct one-on-one supervision such as home visits during each training session, or indirect contact by online telecommunication devices. Further, only few telerehabilitation studies describe interventions using technology-supported devices physically interacting with the patient, although they have the advantage to deliver a high dose and high intensity of training. ${ }^{22-24,}$ ${ }^{28}$ To extend technology-supported training opportunities to the patients homes, these devices should satisfy some general requirements. Many of the developed technologysupported devices so far are bulky and designed for the clinical setting. For independent use at home, technology-supported devices should be easily transportable, safe, usable and user-friendly. ${ }^{17}$ Patients should be able to set up a training session independently. Training at home is likely to be influenced by environmental and psychosocial factors. Ideally, patients should be provided with the possibility of independent training at home with remote (indirect) monitoring including feedback on performance, since meaningful feedback is one of the key principles that supports motor learning. ${ }^{13}$ In addition, maintaining motivation and positive reward can influence the adherence to training, ${ }^{29}$ which is an important factor in telerehabilitation since adherence rates are generally higher in supervised programs. ${ }^{30}$ Therefore, motivational interventions involving a variety of exercises targeted at different types of people need to be developed. There is also an increasing interest using serious games to enhance patients' engagement and adherence in exercise training. ${ }^{31}$ These factors should be taken into account when designing technology-supported training systems for independent practice after stroke. 


\section{SCRIPT PROJECT}

A new type of a technology-supported training device for the arm and hand after stroke was developed within the SCRIPT project: Supervised Care and Rehabilitation Involving Personal Telerobotics. ${ }^{32}$ This training system has the opportunity to easily transport it to the patients' homes, to enable independent practice. This SCRIPT training device (Figure 1.1) involves a passive-actuated orthosis which physically interacted with the arm and hand for support, but also requires active contribution of the patient. Participants were motivated to complete a training session by offering several motivational exercise games, to enhance patients' engagement and adherence to exercise training. Within these motivational games, both arm and hand movements were optimally combined to provide a functional training session. During training, participants received also feedback on performance, to enhance engagement even more. The games were available in a personalized user interface for the patient. Another user interface was available for a healthcare professional to allow remote monitoring, without the need for direct online supervision in which the patient and professional are generally online simultaneously. This distinguishes the SCRIPT training system from many other telerehabilitation approaches after stroke, in which often fixed appointments are made for direct (remote) contact during all training sessions.

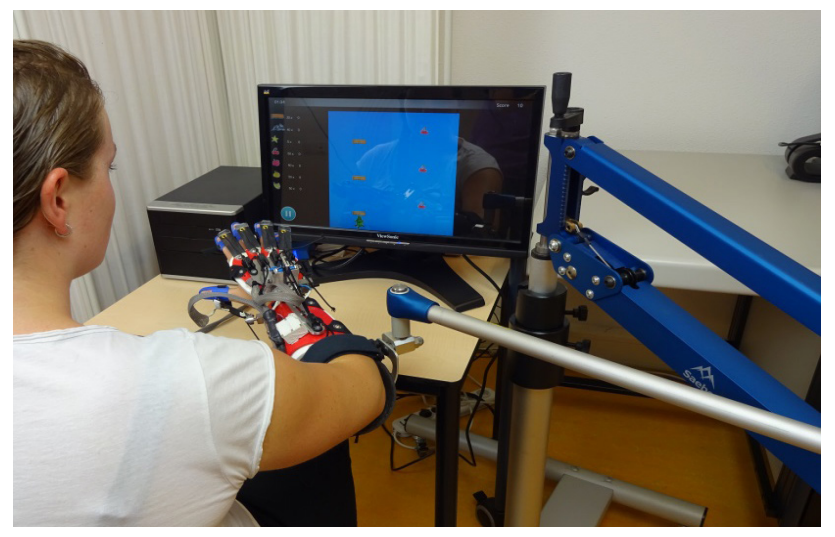

Figure 1.1 SCRIPT training system

The SCRIPT project comprised several clinical and technical partners, all having a different role of the project. Our role as a clinical partner was on the evaluation of the SCRIPT training system in the patients' homes, as described in this thesis. However, before evaluation of the complete training system at home was possible, several preparatory steps were needed. Technical partners were responsible for the development of the SCRIPT training system, with help from the clinical partners on user experience input. The training system was developed using a user-centered iterative cycle design methodology, involving a diverse group of stroke patients, healthcare professionals and technicians to first identify 
user requirements. Addressing stroke survivors' goals, motivations, behavior, feelings and attitude to technologies, by means of performing interviews and home visits, provided meaningful information prior to the actual design of the training system. ${ }^{33}$ Multiple prototypes of the exoskeleton have been evaluated by therapists and stroke patients to improve the orthosis for optimal training at home. ${ }^{34}$ Initially designed games were further developed by improving game control and adaptive mechanisms changing the required movement speed in order to make the exercises neither too easy nor too challenging. ${ }^{35}$ In addition, during the development process the number and type of games were extended by involving different gestures to control the games. ${ }^{36}$ When preparatory steps were finished, the complete SCRIPT training system was evaluated in stroke patients at home. This thesis describes the main results of these evaluations on feasibility and potential clinical effects in two consecutive phases during the iterative design process.

\section{GENERAL AIM}

It is assumed that training at home is a good solution to encourage independent training and consequently enhance a high training dose. The idea is that the use of functional motivational games and feedback would enhance this engagement to training even more. But if patients have the opportunity to use a technology-supported training system at home, do they actually use it? Are they motivated to use the training system? Can they work with the system independently, is it usable? And what are the (direct) effects of using the training system? This thesis aims to evaluate the global impact (in terms of feasibility and potential clinical effects) of self-administered technology-supported, functional training of the arm and hand at home in chronic stroke patients, to enhance independent, motivational and active exercise.

\section{OUTLINE}

In order to first identify promising approaches for training of the arm and hand after stroke using technology-supported devices, a literature review (Chapter 2) was performed. This review provides an overview of training modalities in robot-mediated upper extremity rehabilitation after stroke. It describes robotic control and interaction strategies used in a large number of developed devices for the upper extremity and identifies the most promising approaches. As part of the design phase within the SCRIPT project, we were first interested in the direct effects of a passive dynamic arm and hand orthosis on arm and hand movements, before its clinical potential was examined in a longitudinal feasibility study. Chapter 3 describes the results of these direct effects during the performance of a reach and grasp task in chronic stroke patients. When the first iteration of the complete training system was ready, feasibility was evaluated at home by chronic stroke patients in the Netherlands, Italy and United Kingdom (chapter 4). This complete training system involved both the orthosis in combination with a computerized gaming environment. Results are shown in 
terms of user acceptance, motivation to training, effective use of the training system and clinical changes after training. Next, the use of the SCRIPT training system was compared to a control group performing conventional exercises from an exercise book (chapter 5). This randomized controlled trial compares the results on user acceptance, training duration, and clinical effects between both groups in order to evaluate the additional benefit of one intervention towards another. Besides training-induced changes on clinical outcomes, we were also interested in a more in-depth insight of underlying mechanisms and the role of recovery versus compensation. For that purpose, chapter 6 describes relationships between clinical outcome measures and some more detailed measurements of movement execution parameters, which provides further directions of what treatment applications should target. During the iterative development process, a next, updated version of the training system became ready. Chapter 7 reports the results of this second iteration of the SCRIPT training system evaluated in a new group of chronic stroke patients at home. This second iteration involved some improvements in usability issues found during the use of the first system, among them an updated wrist and hand orthosis and larger variety of games and gestures within the games with respect to the first iteration. This second iteration was again evaluated at feasibility to provide an overview of suggestions for the final design. Finally, in chapter $\mathbf{8}$ the findings of this thesis are discussed and suggestions for future research focusing on independent arm and hand training after stroke and clinical implications are presented.

\section{REFERENCES}

1. Mackay J, Mensah GA, Mendis S and Greenlund K. The Atlas of Heart Disease and Stroke. World Health Organization, 2004.

2. Langhorne P, Bernhardt J and Kwakkel G. Stroke rehabilitation. Lancet. 2011; 377: 1693702.

3. Kwakkel G, Kollen B and Lindeman E. Understanding the pattern of functional recovery after stroke: facts and theories. Restor Neurol Neurosci. 2004; 22: 281-99.

4. Levin MF, Kleim JA and Wolf SL. What do motor "recovery" and "compensation" mean in patients following stroke? NeurorehabilNeural Repair. 2009; 23: 313-9.

5. Krakauer JW. Arm function after stroke: from physiology to recovery. Semin Neurol. 2005; 25: 384-95.

6. Nudo RJ, Plautz EJ and Frost SB. Role of adaptive plasticity in recovery of function after damage to motor cortex. Muscle Nerve. 2001; 24: 1000-19.

7. Taub E, Uswatte G, Mark V and Morris D. The learned nonuse phenomenon: implications for rehabilitation. Eura Medicophys. 2006; 42: 241-55.

8. Taub E and Morris DM. Constraint-induced movement therapy to enhance recovery after stroke. Current atherosclerosis reports. 2001; 3: 279-86.

9. Langhorne $\mathrm{P}$, Coupar $\mathrm{F}$ and Pollock $\mathrm{A}$. Motor recovery after stroke: a systematic review. Lancet Neurol. 2009; 8: 741-54.

10. Veerbeek JM, van Wegen E, van Peppen $R$, et al. What is the evidence for physical therapy poststroke? A systematic review and meta-analysis. PLoS One. 2014; 9: e87987.

11. Hatem SM, Saussez G, della Faille M, et al. Rehabilitation of Motor Function after Stroke: A Multiple Systematic Review Focused on Techniques to Stimulate Upper Extremity 
Recovery. Front Hum Neurosci. 2016; 10: 22.

12. Pollock A, Farmer SE, Brady MC, et al. Interventions for improving upper limb function after stroke. The Cochrane database of systematic reviews. 2014; 11: CD010820.

13. Timmermans AA, Seelen HA, Willmann RD and Kingma H. Technology-assisted training of arm-hand skills in stroke: concepts on reacquisition of motor control and therapist guidelines for rehabilitation technology design. J Neuroeng Rehabil. 2009; 6: 1.

14. Kleim JA and Jones TA. Principles of experience-dependent neural plasticity: implications for rehabilitation after brain damage. J Speech Lang Hear Res. 2008; 51: S225-39.

15. Veerbeek JM, Langbroek-Amersfoort AC, van Wegen EE, Meskers CG and Kwakkel G. Effects of Robot-Assisted Therapy for the Upper Limb After Stroke. Neurorehabil Neural Repair. 2017; 31: 107-21.

16. Norouzi-Gheidari N, Archambault PS and Fung J. Effects of robot-assisted therapy on stroke rehabilitation in upper limbs: systematic review and meta-analysis of the literature. J Rehabil Res Dev. 2012; 49: 479-96.

17. Maciejasz P, Eschweiler J, Gerlach-Hahn K, Jansen-Troy A and Leonhardt S. A survey on robotic devices for upper limb rehabilitation. Journal of Neuroengineering and Rehabilitation. 2014; 11: 3.

18. Mehrholz J, Pohl M, Platz T, Kugler J and Elsner B. Electromechanical and robot-assisted arm training for improving activities of daily living, arm function, and arm muscle strength after stroke. Cochrane Database Syst Rev. 2015; 11: CD006876.

19. Lo AC, Guarino PD, Richards LG, et al. Robot-assisted therapy for long-term upper-limb impairment after stroke. N Engl J Med. 2010; 362: 1772-83.

20. Prange GB, Jannink MJ, Groothuis-Oudshoorn CG, Hermens HJ and ljzerman MJ. Systematic review of the effect of robot-aided therapy on recovery of the hemiparetic arm after stroke. J Rehabil Res Dev. 2006; 43: 171-84.

21. Balasubramanian S, Klein J and Burdet E. Robot-assisted rehabilitation of hand function. Curr Opin Neurol. 2010; 23: 661-70.

22. Laver KE, Schoene D, Crotty M, George S, Lannin NA and Sherrington C. Telerehabilitation services for stroke. Cochrane Database Syst Rev. 2013; 12: CD010255.

23. Chen J, Jin W, Zhang XX, Xu W, Liu XN and Ren CC. Telerehabilitation Approaches for Stroke Patients: Systematic Review and Meta-analysis of Randomized Controlled Trials. J Stroke Cerebrovasc Dis. 2015; 24: 2660-8.

24. Coupar F, Pollock A, Legg LA, Sackley C and van Vliet P. Home-based therapy programmes for upper limb functional recovery following stroke. Cochrane Database Syst Rev. 2012; 5: CD006755.

25. Palmcrantz S, Borg J, Sommerfeld D, et al. An interactive distance solution for stroke rehabilitation in the home setting - A feasibility study. Inform Health Soc Care. 2016: 1-18.

26. Zondervan DK, Friedman N, Chang E, et al. Home-based hand rehabilitation after chronic stroke: Randomized, controlled single-blind trial comparing the MusicGlove with a conventional exercise program. J Rehabil Res Dev. 2016; 53: 457-72.

27. Sivan M, Gallagher J, Makower S, et al. Home-based Computer Assisted Arm Rehabilitation (hCAAR) robotic device for upper limb exercise after stroke: results of a feasibility study in home setting. Journal of Neuroengineering and Rehabilitation. 2014; 11: 163.

28. Johansson T and Wild C. Telerehabilitation in stroke care--a systematic review. J Telemed Telecare. 2011; 17: 1-6.

29. Maeder A, Poultney N, Morgan G and Lippiatt R. Patient Compliance in Home-Based Self-Care Telehealth Projects. J Telemed Telecare. 2015; 21: 439-42.

30. Picorelli AM, Pereira LS, Pereira DS, Felicio D and Sherrington C. Adherence to exercise 
programs for older people is influenced by program characteristics and personal factors: a systematic review. J Physiother. 2014; 60: 151-6.

31. Putrino D. Telerehabilitation and emerging virtual reality approaches to stroke rehabilitation. Curr Opin Neurol. 2014; 27: 631-6.

32. Amirabdollahian F, Ates S, Basteris A, et al. Design, development and deployment of a hand/wrist exoskeleton for home-based rehabilitation after stroke - SCRIPT project. Robotica. 2014; 32: 1331-46.

33. Nasr N, Leon B, Mountain G, et al. The experience of living with stroke and using technology: opportunities to engage and co-design with end users. Disabil Rehabil Assist Technol. 2016; 11: 653-60.

34. Ates S, Haarman CJW and Stienen AHA. SCRIPT passive orthosis: design of interactive hand and wrist exoskeleton for rehabilitation at home after stroke. Autonomous Robots. 2017; 41: 711-23.

35. Basteris A, Nijenhuis SM, Buurke JH, Prange GB and Amirabdollahian F. Lag-lead based assessment and adaptation of exercise speed for stroke survivors. Robotics and Autonomous Systems. 2015; 73: 144-54.

36. Leon B, Basteris A, Infarinato $F$, et al. Grasps recognition and evaluation of stroke patients for supporting rehabilitation therapy. Biomed Res Int. 2014; 2014: 318016. 



\section{Chapter 2}

Training modalities in robot-mediated upper limb rehabilitation in stroke: a framework for classification based on a systematic review

Basteris $A^{*}$, Nijenhuis SM*, Stienen AHA, Buurke JH, Prange GB, Amirabdollahian F Journal of NeuroEngineering and Rehabilitation 2014; 11:111

* Authors contributed equally 


\begin{abstract}
Robot-mediated post-stroke therapy for the upper-extremity dates back to the 1990s. Since then, a number of robotic devices have become commercially available. There is clear evidence that robotic interventions improve upper limb motor scores and strength, but these improvements are often not transferred to performance of activities of daily living. We wish to better understand why. Our systematic review of 74 papers focuses on the targeted stage of recovery, the part of the limb trained, the different modalities used, and the effectiveness of each. The review shows that most of the studies so far focus on training of the proximal arm for chronic stroke patients. About the training modalities, studies typically refer to active, active-assisted and passive interaction. Robot-therapy in active assisted mode was associated with consistent improvements in arm function. More specifically, the use of HRI features stressing active contribution by the patient, such as EMG-modulated forces or a pushing force in combination with spring-damper guidance, may be beneficial.
\end{abstract}

Our work also highlights that current literature frequently lacks information regarding the mechanism about the physical human-robot interaction (HRI). It is often unclear how the different modalities are implemented by different research groups (using different robots and platforms). In order to have a better and more reliable evidence of usefulness for these technologies, it is recommended that the HRI is better described and documented so that work of various teams can be considered in the same group and categories, allowing to infer for more suitable approaches. We propose a framework for categorization of HRI modalities and features that will allow comparing their therapeutic benefits. 


\section{INTRODUCTION}

Stroke is one of the most common causes of adult disabilities. In the United States, approximately 795,000 individuals experience a new or recurrent stroke each year, and the prevalence is estimated at 7,000,000 Americans over 20 years of age. ${ }^{1}$ In Europe, the annual stroke incidence rates are 141.3 per 100,000 in men, and 94.6 in women. ${ }^{2}$ It is expected that the burden of stroke will increase considerably in the next few years. ${ }^{3}$ The high incidence, in combination with an aging society, indicates future increases in incidence, with a strong impact on healthcare services and related costs.

Impairments after stroke can result in a variety of sensory, motor, cognitive and psychological symptoms. The most common and widely recognized impairments after stroke are motor impairments, in most cases affecting the control of movement of the face, arm, and leg on one side of the body, termed as hemiparesis. Common problems in motor function after hemiparetic stroke are muscle weakness, ${ }^{4-6}$ spasticity, ${ }^{4-6}$ increased reflexes, ${ }^{4}$ loss of coordination ${ }^{4,7}$ and apraxia. ${ }^{4}$ Besides, patients may show abnormal muscle co-activation, implicated in stereotyped movement patterns, which is also known as 'flexion synergy' and 'extension synergy'.8, 9 Concerning the upper extremity, impaired arm and hand function contributes considerably to limitations in the ability to perform activities of daily living $(A D L)$. One of the goals of post stroke rehabilitation is to regain arm and hand function, since it is essential to perform activities of daily living independently.

\section{Stroke rehabilitation}

Stroke rehabilitation is often described as a process of active motor relearning that starts within the first few days after stroke. Recovery profiles are characterized by a high interindividual variability, and it occurs in different processes. Some of the first events following nervous system injury are recovery due to restitution of non-infarcted penumbral areas, reduction of edema around the lesion, and resolution of diaschisis, ${ }^{10-12}$ and comprise spontaneous neurological recovery. A longer term mechanism involved in neurological recovery is neuroplasticity, caused by anatomical and functional reorganization of the central nervous system. Additionally, motor recovery after stroke may occur through compensational strategies. Compensation is defined as behavioral substitution, which means that alternative behavioral strategies are adopted to complete a task. In other words, function will be achieved through alternative processes, instead of using processes of 'true recovery' alone. ${ }^{11-13}$

\section{Treatment approaches}

Many treatment approaches have been developed to aid motor recovery after stroke. These interventions are different in their approach to achieve functional gains. For instance, in the 1950s and 1960s, the so-called neurofacilitation approaches were developed. From these 
approaches based on neurophysiological knowledge and theories, the Bobath Concept, or neurodevelopmental treatment (NDT), is the most used approach in Europe. ${ }^{14-16}$ This approach focuses on normalizing muscle tone and movement patterns, guided by a therapist using specific treatment techniques, in order to improve recovery of the hemiparetic side. Gradually, focus shifted towards the motor learning, or relearning approach. ${ }^{17}$ Others have referred to these methods as the task-oriented approach. These new methods of clinical practice are based on the notion that active practice of context-specific motor tasks with suitable feedback will support learning and motor recovery. ${ }^{11,17}$

Overall, there is a lack of convincing evidence to support that any physiotherapy approach is more effective in recovery than any other approach. ${ }^{15,16,18}$ However, in a review of Langhorne et al., ${ }^{19}$ it is stated that some treatments do show promise for improving motor function, particularly those that focus on high-intensity and repetitive task-specific practice. Moreover, research into motor relearning and cortical reorganization after stroke has showed a neurophysiologic basis for important aspects that stimulate restoration of arm function..$^{20-23}$ These important aspects of rehabilitation training involve functional exercises, with high intensity, and with active contribution of the patient in a motivating environment.

\section{Rehabilitation robotics}

Robot-mediated therapy for the upper limb of stroke survivors dates back to the 1990s. Since then a number of robotic devices have become commercially available to clinics and hospitals, for example the InMotion Arm Robot (Interactive Motion Technologies Inc., USA, also known as MIT-Manus) and the Armeo Power (Hocoma, Switzerland). Robotic devices can provide high-intensity, repetitive, task-specific, interactive training. Typically, such robots deliver forces to the paretic limb of the subject while practicing multi-joint gross movements of the arm. Most of the robotic devices applied in clinical trials or clinical practice offer the possibility of choosing among four modalities for training: active, active-assisted, passive and resistive. These terms relate to conventional therapy modes used in clinical practice and refer to subject's status during interaction. Passive training for example refers to subjectpassive/robot-active training such as in continuous passive motion (CPM) devices. The choice of modality (-ies) in each protocol is ultimately made by researchers/therapists.

There is evidence that robotic interventions improve upper limb motor scores and strength, ${ }^{24-26}$ but these improvements are often not transferred to performance of activities of daily living ( $A D L)$. These findings are shared among the most recent studies, including the largest randomized controlled trial related to robot-therapy to date. ${ }^{27} \mathrm{~A}$ possible reason for a limited transfer of motor gains to $A D L$ is that the earlier studies on robot-mediated therapy have only focused on the proximal joints of the arm, while integration of distal with proximal arm training has been recognized as essential to enhance functional gains. ${ }^{28,29}$ 
Another issue involved in the limited transfer of motor gains to ADL improvements in robot-mediated therapy research may relate to the large variety of devices and protocols applied across clinical trials. Lumping together many devices and protocols does not provide knowledge of the effectiveness of individual components, such as which of the available therapeutic modalities result in the largest effect. ${ }^{25}$ Consequently, in literature there has been a transition towards reviews focusing on selective aspects of robot-mediated therapy, rather than its overall effectiveness. ${ }^{30-33}$

A major step in that direction is the description of different control and interaction strategies for robotic movement training. In a non-systematic review, Marchal-Crespo et al. ${ }^{34}$ collected a set of over 100 studies involving both upper and lower limb rehabilitation. They made a first distinction between assistive, challenging and haptic-simulating control strategies. They also described assistive impedance-based controllers, counterbalancing, EMG-based and performance-adapted assistance. Furthermore, they highlighted the need for trials comparing different interaction modalities. However, that review only included articles up to the year 2008 while much more new information has become available in the recent years.

Loureiro et al. ${ }^{35}$ described 16 end-effector and 12 exoskeleton therapy systems in terms of joints involved, degrees of freedom and movements performed. ${ }^{35}$ However, this nonsystematic review did not report about the effects of the interventions or identify the interaction or control strategies used. Specifically focusing on training of the hand, Balasubramanian et al. ${ }^{36}$ identified 30 devices for hand function and described them in terms of degrees of freedom, movements allowed, range of motion, maximum force (torque) and instrumentation. Among these devices, eight showed an improvement in functional use of the affected hand, in terms of increased scores on the Action Research Arm Test, Box and Block test or Wolf Motor Function Test. ${ }^{36}$ In order to maximize improvements on function or even activity level, understanding and specifically targeting mechanisms underlying recovery of the entire upper limb after stroke is essential.

This literature study works towards clarifying the definitions adopted in robotic control and interaction strategies for the hemiparetic upper extremity (including both proximal and distal arm segments), and identifying the most promising approaches. The objective of this systematic review is to explore and identify the human robot interaction mechanisms used by different studies, based on the information provided in literature. We propose a framework to support future categorization of various modalities of human-robot interaction and identify a number of features related to how such strategies are implemented. In addition, we will compare clinical outcome in terms of arm function and activity improvements associated with those interactions, which allows us to identify the most promising types of 
human robot interactions.

\section{Methods}

We conducted a systematic literature search on PubMed with keywords including stroke, robot and arm, upper limb, shoulder, elbow, wrist or hand. Detailed information about the search strategy is provided in Additional file 1: Search strategy. We included full journal papers written in English about robotic training of (any part of) the upper limb. These included either uncontrolled (pre-post design) or (randomized) controlled trials, in which a group of at least four subjects received robot-mediated training. In addition, training outcome must be statistically evaluated (either pre- or post-treatment for the single group or a difference between groups). In cases where results from the same subjects were presented (partially) in other studies (e.g. a pilot study and the definitive protocol) we retained the study with the largest number of participants or the most recent study, if the number of participants were the same. Also, we discarded those studies where other interventions were applied during robot-mediated exercise (e.g. functional electrical stimulation). Two independent reviewers ( $A B$ and $S N$ ) conducted the search and selected the appropriate articles by discarding those articles which did not meet the selection criteria, based on title first, abstract second and subsequently using full-text articles. In case of doubt, the article was included in the next round of selection. After full-text selection, the two reviewers compared their selections for consensus. For each article, only those groups of subjects that were treated with a robotic device were included. Since some studies compared several experimental groups that differed by subject type, device used or experimental protocol, the number of groups did not match the number of articles. Thus, we refer to number of groups rather than number of studies.

For each group we filled a record in a structured table. Since the outcome of an intervention can be influenced by many factors, such as the initial level of impairment or the frequency and duration of the intervention, this table contains an extensive set of information (presented in Additional file 2: Reviewed articles): device used; arm segments involved in training; time post-stroke; number of subjects per group; session duration; number of weeks training; number of sessions per week; total therapy duration; modality (-ies) and features of HRI; baseline impairment measured as average Fugl-Meyer score; and clinical outcome in terms of body functions and activity level. Arm segments involved were categorized as one (single arm segment) or more (multiple arm segments) of shoulder, elbow, forearm, wrist and hand, in which forearm represents pro/supination movement at the radio-ulnar joint. Time since stroke was categorized according to Péter et al., ${ }^{37}$ considering the acute phase as less than three months post stroke, the sub-acute phase as three to six months post stroke and the chronic phase as more than six months post stroke. 
The main focus of this work is on the interaction between the subject and the robot. Table 2.1 categorizes different ways of intervention commonly found in existing robot-mediated therapy (termed as 'training modalities' in this work). In active mode, performance arises from subject contribution only, whereas in passive mode the movement is performed by the robot regardless of subject's response. In assistive modality, both subject and robot contribution affect movement performance. Passive-mirrored mode applies to bimanual devices, when the movement of the affected side is guided based on active performance of the unimpaired side. In active-assisted mode, the subject is performing actively at the beginning of the movement and the robot intervenes only when given conditions are met (e.g. if the target has not been reached within a certain time), leading to systematic success. In corrective mode instead, in such a case the robot would stop the subject to let then reprise active movement. In path guidance mode, the subject is performing actively in the movement direction, and the robot intervention is limited to its orthogonal direction. Finally, in resistive mode, the robot makes the movement more difficult by resisting the movement received from the subject. We categorized each group according to these modalities. Note that some terms refer to the subject status (i.e. "passive" and "active"), others to the robot behavior (e.g. "resistive").

However, these categories are not specific enough to classify such different interventions. For example, in the case of reaching movements, the assistive modality would refer to both cases where the robot is providing weight support or applying target-oriented forces. The terms commonly describing modalities of robot-mediated therapy (such as passive, active-assisted, resistive) had to be revised to provide more specific definitions in order to proceed with unambiguous classification. We therefore categorized all the modalities in a different way, specifically based on the features of their implementation. To do so, we identified the following specific technical features used to implement a certain modality (termed 'HRI features' in this work): passive, passive-mirrored, moving attractor, assistive constant force, triggered assistance, pushing force (in case of delay), EMG-proportional, tunnels, spring-damper guidance, spring and damper against movement. These categories are defined in detail in Table 2.2. Note that neither training modalities nor features of HRI are mutually exclusive categories, since groups might have been tested with several training modalities, and each modality might involve the presence of more than one HRI feature. We propose the classification of training modalities and HRI features adopted in this work as a framework for classification for future studies. This is an open framework, so that as new modalities or features are developed and tested, specific categories could be added, to be further referred. 
Chapter 2

Table 2.1 Training modalities in robot-mediated therapy

\begin{tabular}{ll}
\hline Modality & Specifications \\
\hline Assistive & $\begin{array}{l}\text { Subject's voluntary activity is required during the } \\
\text { entire movement. Robots can assist either providing } \\
\text { weight support or providing forces aiming at task } \\
\text { completion. }\end{array}$ \\
ROBOT
\end{tabular}

Active The robot is being used as a measurement device, without providing force to subject's limb.

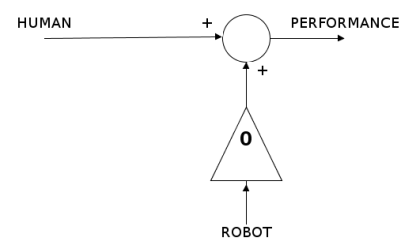

Passive Robot performs the movement without any account of subject's activity.

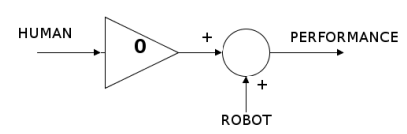

Passive- This is for bimanual robots, when the unimpaired mirrored limb is used to control the passive movement of the affected side.

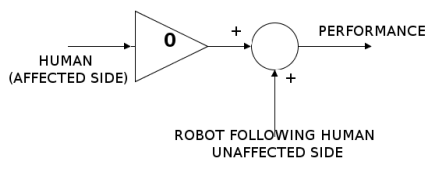

Active- $\quad$ Assistance towards task completion is supplied only assistive when the subject has not been able to perform actively. At this stage, the subject experiences passive movement of the limb.

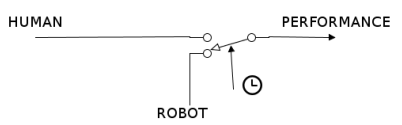

Corrective Subject is stopped by the robot when errors (e.g. distance from a desired position) overcome a predefined value and then asked to perform actively again.

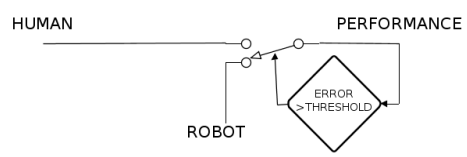

Path Robot guides the subject when deviating from preguidance defined trajectory.

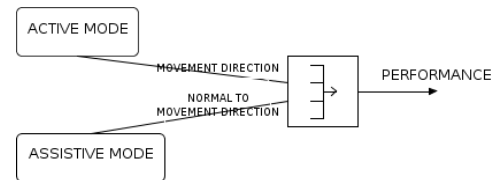

Resistive Robot provides force opposing the movement.

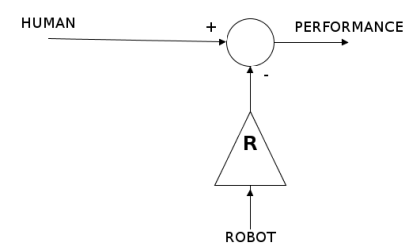


Table 2.2 Features of modalities of human robot interaction and their implementation in robot-mediated therapy

\begin{tabular}{|c|c|}
\hline Feature & Specification \\
\hline Passive, Passive-mirrored & $\begin{array}{l}\text { The device is programmed to follow a desired trajectory/force profile with a } \\
\text { strong attractor (up to } 1000 \mathrm{~N} / \mathrm{m} \text { ) towards it. In the case of passive mirrored, } \\
\text { the desired input is given by the subject with the unimpaired hand. In some } \\
\text { cases, these trajectories can be set by the therapist during a "learning" phase. }\end{array}$ \\
\hline Moving attractor & $\begin{array}{l}\text { In such a case the assistance is lower than in passive control, the robot is still } \\
\text { attracted towards a minimum jerk or smooth trajectory but the amount of } \\
\text { assistance can be modulated by varying the stiffness that attracts the robot } \\
\text { to the trajectory. }\end{array}$ \\
\hline Triggered assistance & $\begin{array}{l}\text { The subject initiates a movement without assistance. The robot observes that } \\
\text { the on-going performance if the task is not completed (e.g. time expired) and } \\
\text { intervenes taking the full control, as in the passive mode. }\end{array}$ \\
\hline Assistive constant force & $\begin{array}{l}\text { Force oriented towards the target or weight support when movement is } \\
\text { against gravity }\end{array}$ \\
\hline EMG-proportional & The power of the EMG signal is used to control the actuators \\
\hline Pushing force (in case of delay) & $\begin{array}{l}\text { A force aligned with the movement direction assists the subject only if there } \\
\text { is a delay in comparison with a scheduled motion pattern }\end{array}$ \\
\hline Spring-damper guidance & $\begin{array}{l}\text { Elastic or visco-elastic force fields aim at reducing the lateral displacement } \\
\text { from a desired trajectory. }\end{array}$ \\
\hline Tunnels & $\begin{array}{l}\text { These can be displaced within the virtual environment to produce a haptic } \\
\text { feedback only if error overcomes a (large) threshold value. A tunnel can be } \\
\text { seen like a lateral spring-damper system plus a dead band zone which makes } \\
\text { the haptic intervention discrete in time. This particular cueing of errors re- } \\
\text { lates to a corrective strategy. }\end{array}$ \\
\hline Spring against movement & $\begin{array}{l}\text { The device opposes movements through an elastic force-field pulling back to } \\
\text { the start position. }\end{array}$ \\
\hline Damper against movement & $\begin{array}{l}\text { The device generates a force opposing the movement based on current ve- } \\
\text { locity. Although this increases the effort of the subject, it also stabilizes the } \\
\text { movement by damping oscillations. }\end{array}$ \\
\hline Not Clear & $\begin{array}{l}\text { The information in the text (or its references) did not allow classifying the } \\
\text { article. As an instance, if the only mention to the physical interaction was "the } \\
\text { robot assisted the subjects during the task", this was considered not clear due } \\
\text { to not providing details on the method of assistance. }\end{array}$ \\
\hline
\end{tabular}

We related clinical outcome to factors as segments of the arm trained, time since stroke and modalities and HRI features. We assessed clinical outcome as whether reported improvements were statistically significant or not, for each measure. Outcome was considered separately for body functions and structures (e.g., Fugl-Meyer, Modified Ashworth Scale, kinematics) and activities (e.g., Action Research Arm Test, Wolf Motor Function Test, Motor Activity Log), according to ICF definitions. ${ }^{38}$ We categorized each outcome measure to either body functions or activities as defined by Sivan et al. ${ }^{39}$ and Salter et al. ${ }^{40-42} \mathrm{~A}$ group was considered to have shown improvement when at least two-thirds of all the outcome measures within a specific category (of either body functions or activity level) had improved significantly. 


\section{RESULTS}

In September 2013, our search led to a total of 423 publications. The first two rounds of filtering, based on title and abstract, led to a set of 126 articles. After screening fulltext articles, 74 studies were included, with a total of 100 groups treated with robots. Of the 74 studies, 35 were randomized controlled trials and 39 were clinical trials (pre-post measurement), involving 36 different devices. Group sizes ranged from 5 to 116 subjects, with a total of 1456 subjects. Table 2.3 presents a summarized overview of all included studies, grouped by device. Detailed information for each group is given in the table in Additional file 2: Reviewed articles.

With respect to stages of stroke recovery (Figure 2.1a), 73 of the 100 groups included patients in the chronic stage, 17 involved patients in the acute stage, and four groups involved patients in the sub-acute stage. In six cases subjects at different stages of recovery were included in the same group or no information about time since stroke was provided. The average FM score at inclusion among groups of acute subjects was $17.7 \pm 12.7$ and 25.9 \pm 9.5 among chronic subjects. The higher average score for subacute subjects $(29.3 \pm 7.8)$ is possibly an outlier due to the small number of observations.

When considering the arm segments (Figure 2.1b), we observed that training involved shoulder movements for 71 groups, elbow flexion-extension for 74 groups, wrist movements for 32 groups, forearm pronation-supination for 20 groups, and hand movements for 20 groups. Training rarely focused on a single part of the arm, with four groups specifically trained for elbow, $86,90,92,93$ five groups for wrist ${ }^{73,85,87,88}$ and six groups for hand ${ }^{76-79,116}$ movements. Training of movements involving the entire upper limb (as those performed during $A D L$ ) is not highly recurrent (seven groups). ${ }^{27,49,84,108,109,113}$

Stage of recovery

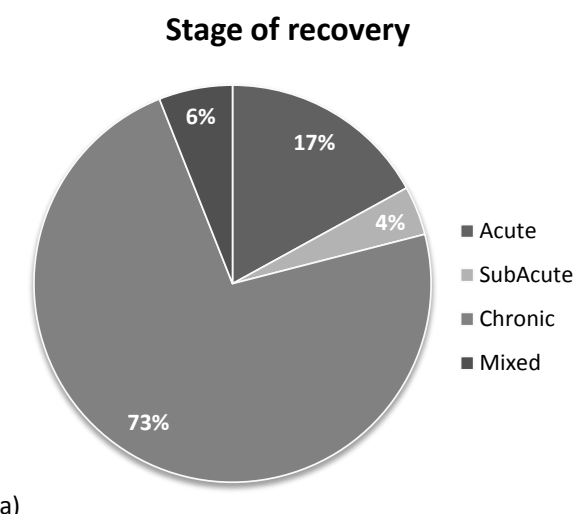

a)
Arm segments

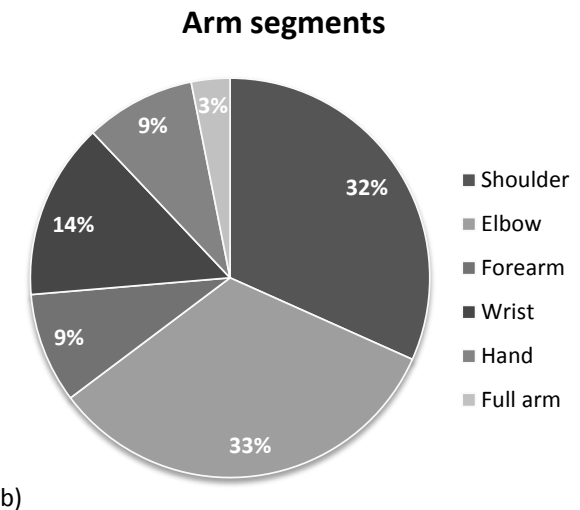

b)

Figure 2.1 Fraction of groups classified by time since stroke (a) and by segments of the arm trained (b) 
Table 2.3 Overview of included studies: characteristics

\begin{tabular}{|c|c|c|c|c|c|c|c|c|}
\hline Device & $\begin{array}{c}\text { Arm } \\
\text { segment }\end{array}$ & Phase & $\begin{array}{c}\text { \# of } \\
\text { groups }\end{array}$ & $\begin{array}{l}\text { Refer- } \\
\text { ences }\end{array}$ & $\begin{array}{c}\text { \# of } \\
\text { subjects } \\
\text { (total) }\end{array}$ & $\begin{array}{c}\text { Training duration } \\
\text { in hours } \\
\text { [mean (SD)] }\end{array}$ & $\begin{array}{l}\text { Training } \\
\text { modalities }\end{array}$ & HRI feature \\
\hline \multirow[t]{2}{*}{$\begin{array}{l}\text { MIT-MANUS } \\
\text { (InMotion2) }\end{array}$} & $S, E$ & Acute & 6 & $43-48$ & 132 & $24.2(10.9)$ & $P, A s, A A, P G$ & $\begin{array}{c}\text { SDG, NC, P, } \\
\text { PF, TA }\end{array}$ \\
\hline & & Chronic & 20 & $48-59$ & 394 & $22.3(17.0)$ & As, AA, R, Ac & $\begin{array}{c}\text { SDG, MA, NC, } \\
\text { PF, S, TA }\end{array}$ \\
\hline $\begin{array}{l}\text { MIT-MANUS } \\
\text { (InMotion2) }\end{array}$ & $\begin{array}{l}S, E, F \\
W, H\end{array}$ & Chronic & 3 & 27,49 & 64 & $24.0(10.4)$ & As, AA & SDG, PF \\
\hline $\begin{array}{c}\text { MIT-MANUS } \\
(\operatorname{InMotion} 2+3)\end{array}$ & $S, E, F, W$ & Chronic & 3 & $59-61$ & 63 & $36.0(0)$ & As, AA, Ac & SDG, NC, PF \\
\hline \multirow[t]{2}{*}{ Bi-Manu-Track } & $\mathrm{F}, \mathrm{W}$ & Acute & 2 & 62,63 & 53 & $10.0(0)$ & $\mathrm{P}, \mathrm{PM}, \mathrm{R}$ & $\mathrm{P}, \mathrm{PM}, \mathrm{S}$ \\
\hline & & Chronic & 5 & $64-67$ & 48 & 26.8 (12.9) & $\mathrm{P}, \mathrm{PM}, \mathrm{R}, \mathrm{Ac}$ & $\mathrm{NC}, \mathrm{P}, \mathrm{PM}, \mathrm{S}$ \\
\hline \multirow[t]{3}{*}{ MIME } & $S, E$ & Acute & 2 & 68 & 36 & $12.2(5.1)$ & $P, P M, A A, R$ & $\mathrm{NC}, \mathrm{P}, \mathrm{PM}, \mathrm{S}$ \\
\hline & & $\begin{array}{l}\text { Sub- } \\
\text { acute }\end{array}$ & 3 & 69 & 24 & $15.0(0)$ & $P, P M, A A, R$ & $\begin{array}{c}\text { SDG, } P, P M, \\
\text { TA, D }\end{array}$ \\
\hline & & Chronic & 4 & $70-72$ & 37 & $24.0(0)$ & $\begin{array}{c}P, P M, A A \\
P G, R\end{array}$ & $\begin{array}{c}\text { SDG, } \mathrm{P}, \mathrm{PM}, \\
\text { TA, D }\end{array}$ \\
\hline $\begin{array}{c}1 \text { DoF robotic } \\
\text { device }\end{array}$ & W & Chronic & 1 & 73 & 8 & $20.0(0)$ & $\mathrm{P}, \mathrm{AA}, \mathrm{A}$ & $\mathrm{P}, \mathrm{TA}$ \\
\hline $\begin{array}{c}2 \text { DoF robotic } \\
\text { device }\end{array}$ & $\mathrm{S}, \mathrm{E}$ & Chronic & 1 & 73 & 12 & $20.0(0)$ & $P, A A, A$ & $\mathrm{P}, \mathrm{TA}$ \\
\hline $\begin{array}{l}3 \text { DoF wrist } \\
\text { robotic exoskel- } \\
\text { eton }\end{array}$ & $\mathrm{F}, \mathrm{W}$ & Chronic & 1 & 74 & 9 & $10.0(0)$ & As, Co, Ac & $M A, D$ \\
\hline $\begin{array}{l}\text { 5DoF industrial } \\
\text { robot }\end{array}$ & $S, E, W$ & Acute & 1 & 75 & 8 & $21.4(0)$ & $\mathrm{P}, \mathrm{AA}, \mathrm{AC}$ & $\mathrm{P}$ \\
\hline \multirow[t]{3}{*}{ Amadeo } & $\mathrm{H}$ & Acute & 2 & 76,77 & 14 & $9.2(5.9)$ & $\mathrm{P}, \mathrm{AA}$ & $\mathrm{P}, \mathrm{NC}$ \\
\hline & & Chronic & 1 & 78 & 12 & $18.0(0)$ & AA & $N C, P$ \\
\hline & & Mixed & 2 & 79 & 15 & $15.0(0)$ & $\mathrm{P}, \mathrm{As}, \mathrm{Ac}$ & $N C, P$ \\
\hline ACT3D & $S, E$ & $\begin{array}{l}\text { Sub- } \\
\text { acute }\end{array}$ & 2 & 80 & 14 & Unknown & As, $\mathrm{R}$ & $A C F$ \\
\hline ARM-Guide & $S, E$ & Chronic & 1 & 81 & 10 & $18.0(0)$ & AA & PF, TA \\
\hline AMES & $\mathrm{W}, \mathrm{H}$ & Chronic & 1 & 82 & 5 & $65.0(0)$ & $\mathrm{P}$ & $\mathrm{P}$ \\
\hline BFIAMT & $S, E$ & Chronic & 1 & 83 & 20 & $12.0(0)$ & $\mathrm{PM}, \mathrm{C}, \mathrm{R}$ & MA, PM, TW \\
\hline $\begin{array}{l}\text { Cyberglove, } \\
\text { Cybergrasp + } \\
\text { Haptic Master }\end{array}$ & $\begin{array}{l}\text { S, E, } \\
W, H\end{array}$ & Chronic & 1 & 84 & 12 & $22.0(0)$ & AA & ACF \\
\hline CYBEX, NORM & W & Chronic & 1 & 85 & $\begin{array}{l}\text { Unknown } \\
\text { (27 in total) }\end{array}$ & Unknown & $\mathrm{P}$ & $P$ \\
\hline PolyJbot & W & Chronic & 1 & 85 & $\begin{array}{l}\text { Unknown } \\
\text { ( } 27 \text { in total) }\end{array}$ & Unknown & $\mathrm{AA}$ & EMG \\
\hline \multirow{2}{*}{$\begin{array}{l}\text { EMG-driven } \\
\text { system }\end{array}$} & $\mathrm{E}$ & Chronic & 1 & 86 & 7 & $30.0(0)$ & $\mathrm{AA}$ & EMG \\
\hline & W & Chronic & 2 & 87,88 & 15 & $42.0(0)$ & As, $A A, R$ & EMG, S \\
\hline
\end{tabular}


Table 2.3 Overview of included studies: characteristics (continued)

\begin{tabular}{|c|c|c|c|c|c|c|c|c|}
\hline Device & $\begin{array}{c}\text { Arm } \\
\text { segment }\end{array}$ & Phase & $\begin{array}{l}\text { \# of } \\
\text { groups }\end{array}$ & $\begin{array}{l}\text { Refer- } \\
\text { ences }\end{array}$ & $\begin{array}{c}\text { \# of } \\
\text { subjects } \\
\text { (total) }\end{array}$ & $\begin{array}{c}\text { Training duration } \\
\text { in hours } \\
\text { [mean (SD)] }\end{array}$ & $\begin{array}{l}\text { Training } \\
\text { modalities }\end{array}$ & HRI feature \\
\hline $\begin{array}{l}\text { EMG-driven } \\
\text { system }\end{array}$ & $\mathrm{H}$ & Chronic & 1 & 89,116 & 10 & $20.0(0)$ & As, Ac & EMG \\
\hline$A J B$ & $\mathrm{E}$ & Chronic & 1 & 90 & 6 & $18.0(0)$ & AA & EMG \\
\hline $\begin{array}{l}\text { Hand mentor } \\
\text { robot system }\end{array}$ & W, H & Mixed & 1 & 91 & 10 & $30.0(0)$ & $P, A A, A c$ & $\mathrm{P}, \mathrm{TA}$ \\
\hline $\begin{array}{l}\text { Myoelectrically } \\
\text { controlled } \\
\text { robotic system }\end{array}$ & $\mathrm{E}$ & Chronic & 2 & 92,93 & 14 & $16.0(5.7)$ & As, $A A, R$ & EMG, S \\
\hline Gentle/S & S, E & Mixed & 2 & 94 & 31 & $9.0(6.4)$ & $\mathrm{P}, \mathrm{AA}, \mathrm{Co}, \mathrm{Ac}$ & $N C, P$ \\
\hline Haptic Knob & $\mathrm{F}, \mathrm{W}, \mathrm{H}$ & Chronic & 1 & 95 & 13 & $18.0(0)$ & $P, A A, R$ & $M A, P$ \\
\hline HWARD & W, H & Chronic & 3 & 96,97 & 36 & $22.8(0.6)$ & $A A, A c$ & $\mathrm{P}, \mathrm{TA}$ \\
\hline Braccio di Ferro & S, E & Chronic & 1 & 98 & 10 & $11.3(0)$ & $\begin{array}{c}P, A A, C o, \\
R, A C\end{array}$ & $C F, T, D$ \\
\hline \multirow[t]{2}{*}{ MEMOS } & S, E & Acute & 1 & 99 & 9 & $16.0(0)$ & $P, A A, A c$ & $\mathrm{P}, \mathrm{TA}$, \\
\hline & & Chronic & 3 & 99-101 & 49 & $16.0(0)$ & $P, A A, A c$ & $\mathrm{P}, \mathrm{TA}$ \\
\hline \multirow[t]{2}{*}{$\begin{array}{l}\text { MEMOS, Brac- } \\
\text { cio di Ferro }\end{array}$} & $S, E$ & $\begin{array}{l}\text { Sub- } \\
\text { acute }\end{array}$ & 1 & 102 & 20 & $15.0(0)$ & $A A, A c$ & TA \\
\hline & & Chronic & 1 & 102 & 21 & $15.0(0)$ & $A A, A C$ & TA \\
\hline REHAROB & S, E & Mixed & 1 & 103 & 15 & $10.0(0)$ & $P$ & $\mathrm{P}$ \\
\hline NeReBot & $S, E, F$ & Acute & 2 & 104,105 & 28 & $18.3(2.4)$ & $\mathrm{P}$, As & $\mathrm{P}, \mathrm{PF}$ \\
\hline $\begin{array}{l}\text { REO }^{\text {TM }} \text { Therapy } \\
\text { System }\end{array}$ & $S, E$ & Acute & 1 & 106 & 10 & $11.3(0)$ & $\mathrm{P}$, As & $N C, P$ \\
\hline ReoGo ${ }^{\text {TM }}$ System & $S, E$ & Chronic & 1 & 107 & 19 & $15.0(0)$ & As, AA, PG, Ac & NC \\
\hline T-WREX & $\begin{array}{l}\text { S, E, } \\
W, H\end{array}$ & Chronic & 2 & 108,109 & 19 & $21.0(4.2)$ & As, Ac & ACF \\
\hline Pneu-WREX & $S, E, H$ & Chronic & 1 & 110 & 13 & $24.0(0)$ & As, AA, Ac & $\mathrm{ACF}, \mathrm{NC}$ \\
\hline $\begin{array}{l}\text { VRROOM, } \\
\text { PHANTOM, } \\
\text { WREX }\end{array}$ & $S, E$ & Chronic & 1 & 111 & 26 & $12.0(0)$ & & \\
\hline UL-EX07 & $S, E, F, W$ & Chronic & 2 & 112 & 10 & $18.0(0)$ & $\mathrm{PM}, \mathrm{As}$ & $\begin{array}{c}\text { PM, MA, SDG, } \\
\text { ACF }\end{array}$ \\
\hline BrightArm & $\begin{array}{l}\text { S, E, } \\
W, H\end{array}$ & Chronic & 1 & 113 & 5 & $12.0(0)$ & As & ACF, NC \\
\hline $\begin{array}{l}\text { Linear shoulder } \\
\text { robot }\end{array}$ & S & Chronic & 1 & 114 & 18 & Unknown & As, AA & $\mathrm{TA}, \mathrm{ACF}$ \\
\hline L-Exos & $S, E, F$ & Chronic & 1 & 115 & 9 & $18.0(0)$ & As, $P G$ & $\mathrm{MA}, \mathrm{ACF}$ \\
\hline
\end{tabular}

Abbreviations: Arm segment: S: Shoulder, E: Elbow, F: Forearm, W: Wrist, H: Hand. Training modalities: P: Passive, PM: Passive-Mirrored, R: Resistive, As: Assistive, AA: Active-Assistive, PG: Path Guidance, Co: Corrective, Ac: Active. Human Robot Interactions (HRI): P: Passive, PM: Passive-Mirrored, S: Spring against movement, MA: Moving attractor, TA: Triggered assistance, PF: Pushing force (in case of delay), EMG: EMG-proportional, T: Tunnels, SDG: Spring-damper guidance, ACF: Assistive constant force, D: Damper against movement, NC: Not Clear. 
We then considered the modalities used, and Figure 2.2 shows an overview of the frequency of usage of each modality. In 63 groups more than one modality was used. Training included active-assistive modality in 63 groups. Twenty-eight groups were trained in assistive modality. Passive training was included in 35 groups. Active and resistive modalities were involved less frequently, in 29 and 22 groups, respectively. The passive-mirrored modality was used in 14 groups, path guidance in seven groups and corrective strategy in five groups.

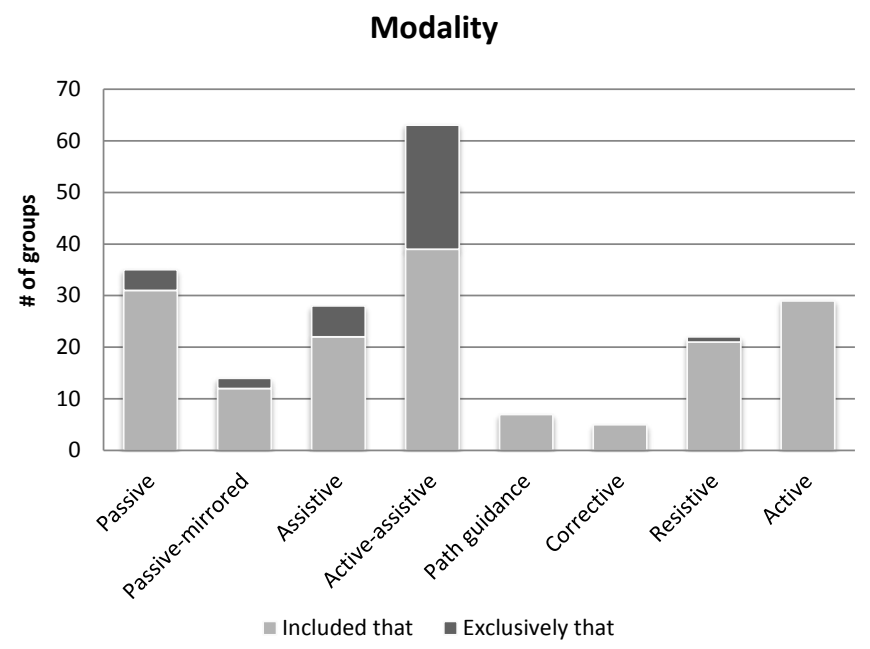

Figure 2.2 Frequency of each modality among the reviewed groups

We also considered these frequencies with respect to the stage of recovery. Passive and passive-mirrored modality are more recurrent for acute than for chronic subjects (with 77 and $24 \%$ of the groups of acute trained with these modalities, versus the respective 21 and $11 \%$ of chronic subjects). Similarly, modalities more suitable for less impaired subjects as resistive and active are more recurrent among chronic (23 and $30 \%$ of the cases, respectively) than within acute subjects ( $18 \%$ for both modalities). Instead, the choice among modalities was not affected by the level of impairment (as measured by FM score). As an instance, subjects trained with passive modality had an average FM of $27.1 \pm 12.8$ at inclusion versus $23.9 \pm 9.6$ of those who did not receive this treatment. Subsequently, we considered the HRI features. In 26 groups there was no clear description or reference to the intervention. Passive and passive mirrored modalities showed the same frequency as reported for the previous classification (35 and 14 groups) as the definition of these categories coincides in the two classifications. Triggered assistance, spring-damper along movement and a pushing force followed in order of frequency after passive training (with respectively 26, 18 and 15 groups). Assistance was delivered as a constant force in twelve groups, as a force proportional to the distance from a moving attractor in seven groups and proportional to the EMG activity in 
eight groups. Resistance was implemented as elastic forces in ten groups and viscous in eight groups. All included studies, except for one, fell in the definitions we provided a priori for categorizations. Recently, a particular paradigm of HRI (error-augmentation) showed clinical benefits, ${ }^{111}$ but it did not fit in any of the modalities we described a priori. In this modality, the robot tends to displace the subject's hand from the optimal trajectory by applying a curl force field to the hand. This constitutes a new, different modality, which benefits could be investigated as more studies using it become available.

We then considered the outcome measures, although the positive outcome of an intervention depends on many factors such as the initial level of impairment of the subjects, frequency and duration of the treatment, baseline impairment (for which detailed information is available in Additional file 2: Reviewed articles). Overall, 54 of 99 groups (55\%) showed significant improvements in body functions. Twenty-two of the 54 groups who measured outcomes related to the activity level (41\%), showed improvements on this level. With respect to time after stroke and observed that among acute stroke patients, 59\% of the groups showed improvements on body functions, and 33\% on activity level. In chronic stroke patients, $53 \%$ of the groups improved on body functions, and $36 \%$ on activity level. For the sub-acute phase, two out of four groups improved on body functions, and two out of three groups improved on activity level.

About outcome for arm segments trained, improvements on body function seemed to be equally distributed between different parts of the arm, but we observed that training of the hand seems to be most effective on the activity levels, with $60 \%$ of the groups showing improvements.

Table 2.4 shows the outcome for different modalities and features of HRI. When relating clinical outcome to specific training modalities, most of the studies (63 of the 100 groups) included multiple modalities in one training protocol. Among them, those including path-guidance (six of the seven groups) and corrective modality (four of the five groups) resulted in the highest percentage of groups improved for body functions ( $86 \%$ and $80 \%$, respectively). However, this may be affected by the limited number of groups. Besides, these groups did not show persistent improvements on activity level. It is noteworthy that the most consistent improvements on activity level were reported for training including the active modality (nine of the 15 groups; $60 \%$ ).

The effect of a single training modality (i.e., only one modality applied in a training protocol) was investigated in the remaining 37 groups: 24 groups applied only the active assistive modality , four groups trained with passive movement only, six groups applied only assistive training, two groups with passive mirrored and one group with resistive modality only. 
Table 2.4 Outcomes per training modality and features of HRI

\begin{tabular}{|c|c|c|c|c|c|c|}
\hline \multirow[b]{2}{*}{ Training modality } & \multicolumn{3}{|c|}{$\%$ of groups improved at body functions } & \multicolumn{3}{|c|}{$\%$ of groups improved at activity level } \\
\hline & $\begin{array}{l}\text { Multiple } \\
\text { modalities }\end{array}$ & $\begin{array}{l}\text { Only that } \\
\text { modality }\end{array}$ & $\begin{array}{l}\text { Features of HRI when } \\
\text { improved }\end{array}$ & $\begin{array}{l}\text { Multiple } \\
\text { modalities }\end{array}$ & $\begin{array}{l}\text { Only that } \\
\text { modality }\end{array}$ & $\begin{array}{l}\text { Features of } H R \\
\text { when improved }\end{array}$ \\
\hline Passive & 56 (19 of 34) & $50(2$ of 4$)$ & $P(2$ of 2$)$ & 44 (10 of 23$)$ & $33(1$ of 3$)$ & $P(1$ of 1$)$ \\
\hline Passive-mirrored & $43(6$ of 14$)$ & $0(0$ of 2$)$ & & 15 (2 of 13$)$ & $0(0$ of 2$)$ & \\
\hline Assistive & $57(16$ of 28$)$ & $33(2$ of 6$)$ & $N C(2$ of 2$)$ & $38(6$ of 16$)$ & $0(0$ of 3$)$ & \\
\hline Active-assistive & $58(36$ of 62$)$ & $58(14$ of 24$)$ & $\begin{array}{l}\text { TA (5 of 14) EMG (4 of } \\
14) \text { PF + SDG ( } 3 \text { of } 14) \\
P F+S D G+N C(1 \text { of } \\
\text { 14) CF (1 of 14) }\end{array}$ & $48(15$ of 31$)$ & $36(4$ of 11$)$ & $\begin{array}{l}\text { TA (2 of 4) CF (1 } \\
\text { of } 4) \text { NC ( } 1 \text { of } 4\end{array}$ \\
\hline Path guidance & $86(6$ of 7$)$ & N/A & & $50(2$ of 4$)$ & $\mathrm{N} / \mathrm{A}$ & \\
\hline Corrective & $80(4$ of 5$)$ & N/A & & $50(1$ of 2$)$ & $\mathrm{N} / \mathrm{A}$ & \\
\hline Resistive & $64(14$ of 22$)$ & $0(0$ of 1$)$ & & $42(5$ of 12$)$ & $\mathrm{N} / \mathrm{A}$ & \\
\hline Active & 61 (17 of 28$)$ & $\mathrm{N} / \mathrm{A}$ & & $60(9$ of 15$)$ & $\mathrm{N} / \mathrm{A}$ & \\
\hline Feature of HRI & $\begin{array}{l}\text { Multiple } \\
\text { features }\end{array}$ & $\begin{array}{l}\text { Only that } \\
\text { feature }\end{array}$ & & $\begin{array}{l}\text { Multiple } \\
\text { features }\end{array}$ & $\begin{array}{l}\text { Only that } \\
\text { feature }\end{array}$ & \\
\hline Passive & 56 (19 of 34) & $60(3$ of 5$)$ & & $44(10$ of 23$)$ & $50(2$ of 4$)$ & \\
\hline Passive-mirrored & $43(6$ of 14$)$ & $0(0$ of 1$)$ & & 15 (2 of 13$)$ & $0(0$ of 1$)$ & \\
\hline Moving attractor & $43(3$ of 7$)$ & $0(0$ of 1$)$ & & $33(2$ of 6$)$ & $0(0$ of 1$)$ & \\
\hline $\begin{array}{l}\text { Triggered } \\
\text { assistance }\end{array}$ & 60 (15 of 25$)$ & 50 (7 of 14$)$ & & $43(6$ of 14$)$ & $44(4$ of 9$)$ & \\
\hline $\begin{array}{l}\text { Assistive Constant } \\
\text { force }\end{array}$ & $42(5$ of 12$)$ & $20(1$ of 5$)$ & & $17(1$ of 6$)$ & $50(1$ of 2$)$ & \\
\hline Emg-proportional & $100(8$ of 8$)$ & 100 (5 of 5$)$ & & $33(1$ of 3$)$ & 33 (1 of 3$)$ & \\
\hline $\begin{array}{l}\text { Pushing force (in } \\
\text { case of delay) }\end{array}$ & $60(9$ of 15$)$ & $\mathrm{N} / \mathrm{A}$ & & $33(2$ of 6$)$ & $\mathrm{N} / \mathrm{A}$ & \\
\hline $\begin{array}{l}\text { Spring-damper } \\
\text { guidance }\end{array}$ & 61 (11 of 18$)$ & N/A & & $38(3$ of 8$)$ & $\mathrm{N} / \mathrm{A}$ & \\
\hline Tunnels or walls & 100 (2 of 2 ) & N/A & & $0(0$ of 1$)$ & $\mathrm{N} / \mathrm{A}$ & \\
\hline $\begin{array}{l}\text { Spring against } \\
\text { movement }\end{array}$ & $60(6$ of 10$)$ & $0(0$ of 1$)$ & & $20(1$ of 5$)$ & $\mathrm{N} / \mathrm{A}$ & \\
\hline $\begin{array}{l}\text { Damper against } \\
\text { movement }\end{array}$ & 75 (6 of 8$)$ & N/A & & $60(3$ of 5$)$ & N/A & \\
\hline Not clear & 46 (12 of 26$)$ & 50 (5 of 10$)$ & & 50 (7 of 14$)$ & $100(2$ of 2$)$ & \\
\hline
\end{tabular}

Abbreviations: Human Robot Interactions (HRI): P: Passive, PM: Passive-Mirrored, S: Spring against movement, MA: Moving attractor, TA: Triggered assistance, PF: Pushing force (in case of delay), EMG: EMG-proportional, T: Tunnels, SDG: Spring-damper guidance, ACF: Assistive constant force, D: Damper against movement, NC: Not Clear.

The active-assisted modality seemed to have the most consistent impact on improvements in both body functions and activities: 14 of the 24 groups $(58 \%)^{43,44,49,60,84,86,88,90,96,100,101}$ showed significant improvements in body functions. Regarding activities, four of the 11 groups $(36 \%)^{43,79,84,96}$ measuring activity level showed significant improvements after active- 
assisted training. Training exclusively in passive mode was associated with improvement in body functions for two of four groups $(50 \%)^{76,103}$ and in activities for one of three groups (33\%). ${ }^{103}$ With the exclusive assistive modality, two of the six groups (33\%) showed significant improvements in body functions. ${ }^{50,61}$ In passive-mirrored mode (two groups) and resistive mode (one group), none of the groups showed significant improvements in either body functions or activities.

We also considered whether the inclusion of a modality led to different outcome for subjects at different phases of recovery. Due to the small number of observations for subacute subjects, we neglect those results. Instead, for acute subjects we found that modalities with better outcome on body functions were active ( 2 out of 3 groups, $67 \%$ ), assistive (4 out of 6 groups, $67 \%$ ), active-assistive (5 out of 10 groups, $50 \%$ ) and passive (6 of 13 groups, $46 \%$ ). For subjects in acute phase, inclusion of passive mirrored and resistive modality did not lead to improvements in body functions (in none of the 4 and 3 groups, respectively). These results differ from subjects in chronic phase, where inclusion of passive-mirrored modality led to improvement in $75 \%$ of the groups ( 6 out of 8 ), while the inclusion of resistive modality was effective on $71 \%$ of the groups (12 of 17). The path guidance modality led to the best results for chronic patients ( 6 out of 6 groups improved on body functions). Results for other modalities are similar among them, with all the other modalities being effective on about $60 \%$ of the groups. The effectiveness is generally lower on the activity level. For acute subjects, there are not many observations for most of the modalities. Instead, for chronic subjects the inclusion of active modality ( $62 \%, 8$ out of 13 groups) seemed to perform better than all the others, which were effective in about $40 \%$ of the cases. Exclusion to this is the passive-mirrored mode, for which only 1 of the 8 groups (12.5\%) improved on activity level.

When we considered the specific HRI features used in the 14 groups who improved on body functions with active-assistance as single modality, five groups used triggered assistance, four groups EMG proportional, four groups a pushing force in combination with springdamper guidance movement, and one group an assistive constant force. The four groups in active-assisted mode who improved on activity level used triggered assistance (two groups), assistive constant force (one group), and for one group it was not clear which feature of HRI was used.

When considering the clinical outcomes associated with those HRI features within the whole studies reviewed, as for the modalities most of the studies included multiple features of HRI in one training protocol (58 groups). Regarding multiple features of HRI applied in training protocols, those including EMG-proportional, tunnels and damper against movement resulted in the highest percentage of groups improved for body functions (100\%, $100 \%$ and $75 \%$, respectively). This is followed by spring damper guidance, pushing force, triggered 
assistance and spring against movement, showing an improvement in body functions in $61 \%$ ( 11 of 18 groups), $60 \%$ (9 of 15 groups), 60\% (15 of 25 groups) and $60 \%$ (6 of 10 groups) respectively. However, the limited number of groups might be affecting this result, especially concerning outcomes at activity level.

The effect of a single feature of HRI (i.e. only one feature of HRI applied in a training protocol) was investigated in the remaining 42 groups. The most common single HRI feature was triggered assistance (TA), which was used in 14 groups. However, only seven of these showed significant improvements in body functions, ${ }^{43,44,96,97,100-102}$ and in the case of activities, four of the nine groups who measured outcomes on activity level (44\%) improved. ${ }^{43,96,97}$ The EMGproportional feature the sole was used in five groups, all showing improved body functions $(100 \%),{ }^{85,86,88,90,116}$ but only one of two groups showed improved activities. ${ }^{116}$ A constant assistive force only was used in five groups, of which only one group showed improvements in both body functions and activities. ${ }^{84}$ So, when focusing on single features, EMG proportional feature seems most promising, followed by passive and triggered assistance. However, the other HRI features that had good results in combined protocols with multiple features (tunnels, damper against movement, spring damper guidance and pushing force) have not been investigated as single feature of HRI at all. Nevertheless, a common aspect can be derived from the features with most consistent effects, indicating that the active component is promising for improving arm function. For activities, no conclusive answers can be drawn, because of the limited number of studies who have investigated this effect using a single feature at this point. This is also true for analysis of the relation of separate training modalities/HRI features with mediating factors such as initial impairment level, frequency and duration of training, etc.

\section{DISCUSSION}

Our results highlight that robot-therapy has focused mostly on subjects in chronic phase of recovery, while considerably less studies involved subjects in acute and in sub-acute phases (73, 17 and four groups, respectively). However, our results indicated that patients across all stages of recovery can benefit from robot-mediated training.

Despite the evidence that training the hand (alone) is accompanied with improvement of both hand and arm function, we observed that many robot-therapy studies focused on proximal rather than on distal arm training. Also, only a limited number of studies focused on training the complete upper limb involving both proximal and distal arm movements, while there is evidence of benefits for training arm and hand together rather than separately. ${ }^{117}$ Additionally, it is known that post-stroke training should include exercises that are as "task-specific/functional" as possible to stimulate motor relearning, which further supports inclusion of the hand and with proximal arm training. ${ }^{28,}{ }^{29}$ Additionally, to allow 
proper investigation of the effect of such functional training of both proximal and distal upper extremity simultaneously, outcome measures at activity level have to be addressed specifically, besides measurements on the level of body functions. However, in all but one study ${ }^{91}$ outcomes related to body function were measured, but the effects of robotic training on activities were assessed in only 54 of 100 groups. This prevents adequate interpretation of the impact of robotic therapy and associated human robot interactions on functional use of the arm at this point.

When focusing on the modality of interaction, we observed that training protocols only occasionally included only one training modality, which makes it difficult to examine the effect of one specific modality. This also hindered a detailed analysis of separate effects per training modality and especially their relation with mediating factors such as initial impairment level, frequency and duration of training, etc.

Only a limited number of studies aimed at comparing two or more different robotic treatments. The first of those studies hypothesized benefits of inserting phases of resistive training in the therapy protocol. ${ }^{51}$ Subjects were assigned to different groups, training with active-assistive modality only, resistive only or both. There were no significant effects from incorporating resistance exercises. Another study ${ }^{69}$ compared a bimanual therapy (in passive mirrored mode) with a unimanual protocol which included passive, active-assistive and resistive training. Again, there were no significant differences between groups in terms of clinical scores. In a different study, active-assistive training delivered with an EMG-controlled device showed larger improvements (in Fugl-Meyer, Modified Ashworth Scale and muscle coordination) with respect to passive movement in wrist training. ${ }^{85}$ Assistive forces may also be provided as weight support. In this case, subjects benefitted from a progressive decrease of such assistance, along therapy. ${ }^{80}$

Even though there are a limited number of studies comparing separate training modalities, the available data indicated that robot-mediated therapy in active assisted mode led most consistently to improvements in arm function. Whether this mode is actually the most effective one cannot be stated at this point due to lack of a standard definitions used by different studies. It is remarkable that the application of two of the least adopted modalities, i.e. path guidance and corrective, did consistently result in improved clinical outcome. Although this effect might be due to the small number of studies which included them, this suggests that one way to be pursued in future research in order to improve the results of robotic rehabilitation is utilizing robot's programmable interaction potentials, rather than just mimicking what a therapist can do (passive, active-assisted, even resistance to some extent). Experimental protocols including more than one modality should also be sought, and the combined effect of different modalities should be investigated. As an instance, 
patients might switch from passive toward active modality as recovery progresses.

With respect to the specific strategy (HRI feature) for providing assistance when applying active assistive training, the findings from this review indicate that a pushing force in combination with lateral spring damper, or EMG-modulated assistance were associated with consistent improvements in arm function across studies, while triggered assistance showed less consistent improvement. It is suggested that modalities that stress the active nature of an exercise, requiring patients to initiate movements by themselves and keep being challenged in a progressive way throughout training (i.e., taking increases in arm function into account by increasing the level of active participation required during robottherapy), do show favorable results on body function level.

The training modalities referred mainly to the description given by the authors of the reviewed studies, but in absence of a uniform definition for identifying robot-human contributions, groups often named the mechanisms used according to their preference and understanding of these mechanisms. Attention to the interaction mechanism between a person and robot is sometimes so limited that often authors did not even mention such mechanisms in their publications (this happened for 26 out of 100 groups). Given a commonly accepted categorization for these modalities, researchers are then able to compare usefulness of different human-robot interaction mechanisms. It is thought that by providing a commonbase for interaction, a larger body of evidence can be provided, i.e. via a data-sharing paradigm, to understand the full potential of robot-mediated therapy for improving arm and hand function after stroke. Ultimately, this can support better integration of robot-mediated therapy in day-to-day therapeutic interventions.

About the effectiveness of different modalities, considering that cortical reorganization and outcome after stroke rehabilitation is positively associated with active, repetitive taskspecific (i.e., functional) practice, ${ }^{28,} 29$ human robot interactions stressing these features are preferred. In addition, there is a strong computational basis to push towards delivering minimally assistive therapy. ${ }^{118}$ In contrast to this, passive movement is very recurrent, as it may provide more severely impaired subjects with the opportunity to practice. If this is the case, one way to improve the outcome of the therapy in this situation is to tailor the exercise to individual needs while stimulating active contribution of the patient as much as possible, rather than passively guiding to systematic success. Nevertheless, due to the large variety and heterogeneity in training modalities applied (i.e. contents of the intervention), it wasn't possible to draw conclusions about the role of these additional mediating factors per training modality. More research about comparing different training modalities (with only one specific modality per group) is needed to answer more specifically which training modality would result in largest improvements in arm function and activities after robot- 
mediated upper limb training after stroke.

\section{CONCLUSIONS}

Our review shows that most of the literature about robot-mediated therapy for stroke survivors refers to subjects in chronic phase. In the same way, training most frequently targeted the proximal arm. Regarding the human-robot interaction, there has been poor attention in documenting the control strategy and identifying which strategy provides better results. This is hampered by the ambiguity in definitions of HRI method employed. While each robot would incorporate a lower-level control, interaction between robot and human is often made possible by incorporating an interaction modality, which is not standardized across different studies. Therefore, we have proposed a categorization of training modalities (Table 2.1) and features of human-robot interaction (Table 2.2) as an open framework to allow groups to identify the type of mechanism used in their studies, allowing better comparison of results for particular training modalities.

Even though only limited studies specifically investigated different control strategies, the present review indicated that robot-therapy in active assisted mode was highly predominant in the available studies and was associated with consistent improvements in arm function. More specifically, the use of HRI features stressing active contribution by the patient (e.g. EMG-modulated control, or a pushing force in combination with spring-damper guidance) in robot-mediated upper limb training after stroke may be beneficial. More research into comparing separate training modalities is needed to identify which specific training modality is associated best with improvements in arm function and activities after robot-mediated upper limb training after stroke.

\section{REFERENCES}

1. Roger VL, Go AS, Lloyd-Jones DM, et al. Heart disease and stroke statistics--2012 update: a report from the American Heart Association. Circulation. 2012; 125: e2-e220.

2. Heuschmann PU, Di Carlo A, Bejot Y, et al. Incidence of stroke in Europe at the beginning of the 21st century. Stroke. 2009; 40: 1557-63.

3. Heidenreich PA, Trogdon JG, Khavjou OA, et al. Forecasting the future of cardiovascular disease in the United States: a policy statement from the American Heart Association. Circulation. 2011; 123: 933-44.

4. Kelly-Hayes M, Robertson JT, Broderick JP, et al. The American Heart Association Stroke Outcome Classification. Stroke. 1998; 29: 1274-80.

5. Wolfe CD. The impact of stroke. Br Med Bull. 2000; 56: 275-86.

6. Teasell R. Musculoskeletal complications of hemiplegia following stroke. Semin Arthritis Rheum. 1991; 20: 385-95.

7. Trombly CA. Deficits of reaching in subjects with left hemiparesis: a pilot study. Am J Occup Ther. 1992; 46: 887-97.

8. Twitchell TE. The restoration of motor function following hemiplegia in man. Brain. 1951; 74: 443-80. 
9. Brunnström S. Movement therapy in hemiplegia: a neurophysiological approach. New York: Medical Dept., Harper \& Row, 1970.

10. Chen R, Cohen LG and Hallett M. Nervous system reorganization following injury. Neuroscience. 2002; 111: 761-73.

11. Shumway-Cook A and Woollacott MH. Motor Control: Translating Research Into Clinical Practice. 3 ed. Philadelphia: Lippincott Williams \& Wilkins, 2007.

12. Kwakkel G, Kollen B and Lindeman E. Understanding the pattern of functional recovery after stroke: facts and theories. Restor Neurol Neurosci. 2004; 22: 281-99.

13. Cirstea MC and Levin MF. Compensatory strategies for reaching in stroke. Brain. 2000; 123 ( Pt 5): 940-53.

14. Lennon S, Baxter D and Ashburn A. Physiotherapy based on the Bobath concept in stroke rehabilitation: a survey within the UK. DisabilRehabil. 2001; 23: 254-62.

15. Pollock A, Baer G, Langhorne P and Pomeroy V. Physiotherapy treatment approaches for the recovery of postural control and lower limb function following stroke: a systematic review. Clin Rehabil. 2007; 21: 395-410.

16. van Peppen RPS, Kwakkel G, Harmeling-van der Wel BC, et al. Guideline for stroke by KNGF [in Dutch: KNGF-richtlijn Beroerte]. Nederlands Tijdschrift voor Fysiotherapie. 2004; 114: 1-78.

17. Carr JH and Shepherd RB. A motor relearning programme for stroke. 2 ed. Rockville, MD: Aspen Publishers, 1987.

18. Liao JY and Kirsch RF. Predicting the initiation of minimum-jerk submovements in threedimensional target-oriented human arm trajectories. Conf Proc IEEE Eng Med Biol Soc. 2012; 2012: 6797-800.

19. Langhorne $P$, Coupar $F$ and Pollock $A$. Motor recovery after stroke: a systematic review. Lancet Neurol. 2009; 8: 741-54.

20. Schaechter JD. Motor rehabilitation and brain plasticity after hemiparetic stroke. Progress in Neurobiology. 2004; 73: 61-72.

21. Krakauer JW. Arm function after stroke: from physiology to recovery. Semin Neurol. 2005; 25: 384-95.

22. Kwakkel G. Intensity of practice after stroke: More is better. Schweizer Archiv für Neurologie und Psychiatrie. 2009; 7: 295-8.

23. Kwakkel G, van Peppen R, Wagenaar RC, et al. Effects of augmented exercise therapy time after stroke: a meta-analysis. Stroke. 2004; 35: 2529-39.

24. Mehrholz J, Pohl M, Platz T, Kugler J and Elsner B. Electromechanical and robot-assisted arm training for improving activities of daily living, arm function, and arm muscle strength after stroke. Cochrane Database Syst Rev. 2015; 11: CD006876.

25. Prange GB, Jannink MJ, Groothuis-Oudshoorn CG, Hermens HJ and ljzerman MJ. Systematic review of the effect of robot-aided therapy on recovery of the hemiparetic arm after stroke. J Rehabil Res Dev. 2006; 43: 171-84.

26. Kwakkel G, Kollen BJ and Krebs HI. Effects of robot-assisted therapy on upper limb recovery after stroke: a systematic review. Neurorehabil Neural Repair. 2008; 22: 11121.

27. Lo AC, Guarino PD, Richards LG, et al. Robot-assisted therapy for long-term upper-limb impairment after stroke. N Engl J Med. 2010; 362: 1772-83.

28. Oujamaa L, Relave I, Froger J, Mottet D and Pelissier JY. Rehabilitation of arm function after stroke. Literature review. Ann Phys Rehabil Med. 2009; 52: 269-93.

29. Timmermans AA, Seelen HA, Willmann RD and Kingma H. Technology-assisted training of arm-hand skills in stroke: concepts on reacquisition of motor control and therapist guidelines for rehabilitation technology design. J Neuroeng Rehabil. 2009; 6: 1. 
30. Brochard S, Robertson J, Medee B and Remy-Neris O. What's new in new technologies for upper extremity rehabilitation? Curr Opin Neurol. 2010; 23: 683-7.

31. Huang VS and Krakauer JW. Robotic neurorehabilitation: a computational motor learning perspective. J Neuroeng Rehabil. 2009; 6: 5.

32. Waldner A, Tomelleri C and Hesse S. Transfer of scientific concepts to clinical practice: recent robot-assisted training studies. Funct Neurol. 2009; 24: 173-7.

33. Pignolo L. Robotics in neuro-rehabilitation. J Rehabil Med. 2009; 41: 955-60.

34. Marchal-Crespo L and Reinkensmeyer DJ. Review of control strategies for robotic movement training after neurologic injury. J Neuroeng Rehabil. 2009; 6: 20.

35. Loureiro RC, Harwin WS, Nagai K and Johnson M. Advances in upper limb stroke rehabilitation: a technology push. Med Biol Eng Comput. 2011; 49: 1103-18.

36. Balasubramanian S, Klein J and Burdet E. Robot-assisted rehabilitation of hand function. Curr Opin Neurol. 2010; 23: 661-70.

37. Péter O, Fazekas G, Zsiga K and Dénes Z. Robot-mediated upper limb physiotherapy: review and recommendations for future clinical trials. Int J Rehabil Res. 2011; 34: 196202.

38. WHO. International Classification of Functioning, Disability and Health (ICF). Geneva: WHO, 2001.

39. Sivan M, O'Connor RJ, Makower S, Levesley M and Bhakta B. Systematic review of outcome measures used in the evaluation of robot-assisted upper limb exercise in stroke. J Rehabil Med. 2011; 43: 181-9.

40. Salter K, Jutai JW, Teasell R, Foley NC and Bitensky J. Issues for selection of outcome measures in stroke rehabilitation: ICF Body Functions. DisabilRehabil. 2005; 27: 191207.

41. Salter K, Jutai JW, Teasell R, Foley NC, Bitensky J and Bayley M. Issues for selection of outcome measures in stroke rehabilitation: ICF activity. DisabilRehabil. 2005; 27: 31540.

42. Salter K, Jutai JW, Teasell R, Foley NC, Bitensky J and Bayley M. Issues for selection of outcome measures in stroke rehabilitation: ICF Participation. DisabilRehabil. 2005; 27: 507-28.

43. Fasoli SE, Krebs HI, Ferraro M, Hogan N and Volpe BT. Does shorter rehabilitation limit potential recovery poststroke? Neurorehabil Neural Repair. 2004; 18: 88-94.

44. Duret $\mathrm{C}$ and Hutin $\mathrm{E}$. Effects of prolonged robot-assisted training on upper limb motor recovery in subacute stroke. NeuroRehabilitation. 2013; 33: 41-8.

45. Aisen ML, Krebs HI, Hogan N, McDowell F and Volpe BT. The effect of robot-assisted therapy and rehabilitative training on motor recovery following stroke. Arch Neurol. 1997; 54: 443-6.

46. Volpe BT, Krebs HI, Hogan N, Edelstein OL, Diels C and Aisen M. A novel approach to stroke rehabilitation: robot-aided sensorimotor stimulation. Neurology. 2000; 54: 1938-44.

47. Rabadi $M$, Galgano $M$, Lynch $D$, Akerman $M$, Lesser $M$ and Volpe B. A pilot study of activity-based therapy in the arm motor recovery post stroke: a randomized controlled trial. Clin Rehabil. 2008; 22: 1071-82.

48. Dipietro L, Krebs HI, Volpe BT, et al. Learning, not adaptation, characterizes stroke motor recovery: evidence from kinematic changes induced by robot-assisted therapy in trained and untrained task in the same workspace. IEEE Trans Neural Syst Rehabil Eng. 2012; 20: 48-57.

49. Krebs HI, MernoffS, Fasoli SE, Hughes R, Stein J and Hogan N. A comparison of functional and impairment-based robotic training in severe to moderate chronic stroke: a pilot study. NeuroRehabilitation. 2008; 23: 81-7. 
50. Krebs HI, Ferraro M, Buerger SP, et al. Rehabilitation robotics: pilot trial of a spatial extension for MIT-Manus. J Neuroeng Rehabil. 2004; 1: 5.

51. Stein J, Krebs HI, Frontera WR, Fasoli SE, Hughes R and Hogan N. Comparison of two techniques of robot-aided upper limb exercise training after stroke. Am J Phys Med Rehabil. 2004; 83: 720-8.

52. Ferraro M, Palazzolo JJ, Krol J, Krebs HI, Hogan N and Volpe BT. Robot-aided sensorimotor arm training improves outcome in patients with chronic stroke. Neurology. 2003; 61: 1604-7.

53. Daly JJ, Hogan N, Perepezko EM, et al. Response to upper-limb robotics and functional neuromuscular stimulation following stroke. J Rehabil Res Dev. 2005; 42: 723-36.

54. Macclellan LR, Bradham DD, Whitall J, et al. Robotic upper-limb neurorehabilitation in chronic stroke patients. J Rehabil Res Dev. 2005; 42: 717-22.

55. Finley MA, Fasoli SE, Dipietro L, et al. Short-duration robotic therapy in stroke patients with severe upper-limb motor impairment. J Rehabil Res Dev. 2005; 42: 683-92.

56. Volpe BT, Lynch D, Rykman-Berland A, et al. Intensive sensorimotor arm training mediated by therapist or robot improves hemiparesis in patients with chronic stroke. Neurorehabil Neural Repair. 2008; 22: 305-10.

57. Posteraro F, Mazzoleni S, Aliboni S, et al. Upper limb spasticity reduction following active training: a robot-mediated study in patients with chronic hemiparesis. J Rehabil Med. 2010; 42: 279-81.

58. Conroy SS, Whitall J, Dipietro L, et al. Effect of gravity on robot-assisted motor training after chronic stroke: a randomized trial. Arch Phys Med Rehabil. 2011; 92: 1754-61.

59. Mazzoleni S, Sale P, Franceschini M, et al. Effects of proximal and distal robot-assisted upper limb rehabilitation on chronic stroke recovery. NeuroRehabilitation. 2013; 33: 33-9.

60. Zollo L, Rossini L, Bravi M, Magrone G, Sterzi S and Guglielmelli E. Quantitative evaluation of upper-limb motor control in robot-aided rehabilitation. Med Biol Eng Comput. 2011; 49: 1131-44.

61. Pellegrino G, Tomasevic L, Tombini M, et al. Inter-hemispheric coupling changes associate with motor improvements after robotic stroke rehabilitation. Restor Neurol Neurosci. 2012; 30: 497-510.

62. Hesse S, Waldner A, Mehrholz J, Tomelleri C, Pohl M and Werner C. Combined transcranial direct current stimulation and robot-assisted arm training in subacute stroke patients: an exploratory, randomized multicenter trial. Neurorehabil Neural Repair. 2011; 25: 838-46.

63. Hesse S, Werner C, Pohl M, Rueckriem S, Mehrholz J and Lingnau ML. Computerized arm training improves the motor control of the severely affected arm after stroke: a single-blinded randomized trial in two centers. Stroke. 2005; 36: 1960-6.

64. Hsieh YW, Wu CY, Liao WW, Lin KC, Wu KY and Lee CY. Effects of treatment intensity in upper limb robot-assisted therapy for chronic stroke: a pilot randomized controlled trial. Neurorehabil Neural Repair. 2011; 25: 503-11.

65. Hesse S, Schulte-Tigges G, Konrad M, Bardeleben A and Werner C. Robot-assisted arm trainer for the passive and active practice of bilateral forearm and wrist movements in hemiparetic subjects. Arch Phys Med Rehabil. 2003; 84: 915-20.

66. Liao WW, Wu CY, Hsieh YW, Lin KC and Chang WY. Effects of robot-assisted upper limb rehabilitation on daily function and real-world arm activity in patients with chronic stroke: a randomized controlled trial. Clin Rehabil. 2012; 26: 111-20.

67. Wu CY, Yang CL, Chuang LL, et al. Effect of therapist-based versus robot-assisted bilateral arm training on motor control, functional performance, and quality of life after chronic stroke: a clinical trial. Phys Ther. 2012; 92: 1006-16. 
68. Burgar CG, Lum PS, Scremin AM, et al. Robot-assisted upper-limb therapy in acute rehabilitation setting following stroke: Department of Veterans Affairs multisite clinical trial. J Rehabil Res Dev. 2011; 48: 445-58.

69. Lum PS, Burgar CG, Van der Loos M, Shor PC, Majmundar M and Yap R. MIME robotic device for upper-limb neurorehabilitation in subacute stroke subjects: A follow-up study. J Rehabil Res Dev. 2006; 43: 631-42.

70. Burgar CG, Lum PS, Shor PC and Machiel Van der Loos HF. Development of robots for rehabilitation therapy: the Palo Alto VA/Stanford experience. J Rehabil Res Dev. 2000; 37: 663-73.

71. Lum PS, Burgar CG, Shor PC, Majmundar M and Van der Loos M. Robot-assisted movement training compared with conventional therapy techniques for the rehabilitation of upper-limb motor function after stroke. Arch Phys Med Rehabil. 2002; 83: 952-9.

72. Lum PS, Burgar CG and Shor PC. Evidence for improved muscle activation patterns after retraining of reaching movements with the MIME robotic system in subjects with poststroke hemiparesis. IEEE Trans Neural Syst Rehabil Eng. 2004; 12: 186-94.

73. Colombo R, Pisano F, Mazzone A, et al. Design strategies to improve patient motivation during robot-aided rehabilitation. J Neuroeng Rehabil. 2007; 4: 3.

74. Squeri V, Masia L, Giannoni P, Sandini G and Morasso P. Wrist rehabilitation in chronic stroke patients by means of adaptive, progressive robot-aided therapy. IEEE Trans Neural Syst Rehabil Eng. 2014; 22: 312-25.

75. Abdullah HA, Tarry C, Lambert C, Barreca S and Allen BO. Results of Clinicians Using a Therapeutic Robotic System in an Inpatient Stroke Rehabilitation Unit. Journal of Neuroengineering and Rehabilitation. 2011; 8.

76. Pinter D, Pegritz S, Pargfrieder C, et al. Exploratory study on the effects of a robotic hand rehabilitation device on changes in grip strength and brain activity after stroke. Top Stroke Rehabil. 2013; 20: 308-16.

77. Sale P, Lombardi V and Franceschini M. Hand robotics rehabilitation: feasibility and preliminary results of a robotic treatment in patients with hemiparesis. Stroke Res Treat. 2012; 2012: 820931.

78. Stein J, Bishop L, Gillen G and Helbok R. Robot-Assisted Exercise for Hand Weakness After Stroke: A Pilot Study. Am J Phys Med Rehabil. 2011.

79. Hwang $\mathrm{CH}$, Seong JW and Son DS. Individual finger synchronized robot-assisted hand rehabilitation in subacute to chronic stroke: a prospective randomized clinical trial of efficacy. Clin Rehabil. 2012.

80. Ellis MD, Sukal-Moulton T and Dewald JP. Progressive shoulder abduction loading is a crucial element of arm rehabilitation in chronic stroke. Neurorehabil Neural Repair. 2009; 23: 862-9.

81. Kahn LE, Lum PS, Rymer WZ and Reinkensmeyer DJ. Robot-assisted movement training for the stroke-impaired arm: Does it matter what the robot does? J Rehabil Res Dev. 2006; 43: 619-30.

82. Cordo P, Lutsep H, Cordo L, Wright WG, Cacciatore T and Skoss R. Assisted movement with enhanced sensation (AMES): coupling motor and sensory to remediate motor deficits in chronic stroke patients. Neurorehabil Neural Repair. 2009; 23: 67-77.

83. Chang JJ, Tung WL, Wu WL, Huang MH and Su FC. Effects of robot-aided bilateral forceinduced isokinetic arm training combined with conventional rehabilitation on arm motor function in patients with chronic stroke. Arch Phys Med Rehabil. 2007; 88: 13328.

84. Merians AS, Fluet GG, Qiu Q, et al. Robotically facilitated virtual rehabilitation of arm transport integrated with finger movement in persons with hemiparesis. J Neuroeng 
Rehabil. 2011; 8: 27.

85. Wei XJ, Tong KY and Hu XL. The responsiveness and correlation between Fugl-Meyer Assessment, Motor Status Scale, and the Action Research Arm Test in chronic stroke with upper-extremity rehabilitation robotic training. Int J Rehabil Res. 2011; 34: 349-56.

86. Hu X, Tong KY, Song R, Tsang VS, Leung PO and Li L. Variation of muscle coactivation patterns in chronic stroke during robot-assisted elbow training. Arch Phys Med Rehabil. 2007; 88: 1022-9.

87. Song R, Tong KY, Hu X and Zhou W. Myoelectrically controlled wrist robot for stroke rehabilitation. J Neuroeng Rehabil. 2013; 10: 52.

88. Hu XL, Tong KY, Song R, et al. Quantitative evaluation of motor functional recovery process in chronic stroke patients during robot-assisted wrist training. J Electromyogr Kinesiol. 2009; 19: 639-50.

89. Hu XL, Tong KY, Li R, Xue JJ, Ho SK and Chen P. The effects of electromechanical wrist robot assistive system with neuromuscular electrical stimulation for stroke rehabilitation. $J$ Electromyogr Kinesiol. 2012.

90. Stein J, Narendran K, McBean J, Krebs K and Hughes R. Electromyography-controlled exoskeletal upper-limb-powered orthosis for exercise training after stroke. Am J Phys Med Rehabil. 2007; 86: 255-61.

91. Kutner NG, Zhang R, Butler AJ, Wolf SL and Alberts JL. Quality-of-life change associated with robotic-assisted therapy to improve hand motor function in patients with subacute stroke: a randomized clinical trial. Phys Ther. 2010; 90: 493-504.

92. Song $\mathrm{R}$, Tong $\mathrm{KY}, \mathrm{Hu} \mathrm{X}$ and $\mathrm{Li} \mathrm{L}$. Assistive control system using continuous myoelectric signal in robot-aided arm training for patients after stroke. IEEE Trans Neural Syst Rehabil Eng. 2008; 16: 371-9.

93. Song R, Tong KY, Hu X, Li L and Sun R. Arm-eye coordination test to objectively quantify motor performance and muscles activation in persons after stroke undergoing robotaided rehabilitation training: a pilot study. Exp Brain Res. 2013; 229: 373-82.

94. Amirabdollahian F, Loureiro R, Gradwell E, Collin C, Harwin W and Johnson G. Multivariate analysis of the Fugl-Meyer outcome measures assessing the effectiveness of GENTLE/S robot-mediated stroke therapy. J Neuroeng Rehabil. 2007; 4: 4.

95. Lambercy $\mathrm{O}$, Dovat $\mathrm{L}$, Yun $\mathrm{H}$, et al. Effects of a robot-assisted training of grasp and pronation/supination in chronic stroke: a pilot study. J Neuroeng Rehabil. 2011; 8: 63.

96. Takahashi CD, Der-Yeghiaian L, Le V, Motiwala RR and Cramer SC. Robot-based hand motor therapy after stroke. Brain. 2008; 131: 425-37.

97. Riley JD, Le V, Der-Yeghiaian L, et al. Anatomy of stroke injury predicts gains from therapy. Stroke. 2011; 42: 421-6.

98. Casadio M, Giannoni P, Morasso P and Sanguineti V. A proof of concept study for the integration of robot therapy with physiotherapy in the treatment of stroke patients. Clin Rehabil. 2009; 23: 217-28.

99. Colombo R, Pisano F, Micera S, et al. Assessing mechanisms of recovery during robotaided neurorehabilitation of the upper limb. Neurorehabil Neural Repair. 2008; 22: 50 63.

100. Colombo R, Sterpi I, Mazzone A, Delconte C, Minuco G and Pisano F. Measuring changes of movement dynamics during robot-aided neurorehabilitation of stroke patients. IEEE Trans Neural Syst Rehabil Eng. 2010; 18: 75-85.

101. Panarese A, Colombo R, Sterpi I, Pisano F and Micera S. Tracking motor improvement at the subtask level during robot-aided neurorehabilitation of stroke patients. Neurorehabil Neural Repair. 2012; 26: 822-33.

102. Colombo R, Sterpi I, Mazzone A, Delconte Cand Pisano F. Robot-aided neurorehabilitation in sub-acute and chronic stroke: does spontaneous recovery have a limited impact on 
outcome? NeuroRehabilitation. 2013; 33: 621-9.

103. Fazekas G, Horvath M, Troznai T and Toth A. Robot-mediated upper limb physiotherapy for patients with spastic hemiparesis: a preliminary study. J Rehabil Med. 2007; 39: 580-2.

104. Masiero S, Armani M and Rosati G. Upper-limb robot-assisted therapy in rehabilitation of acute stroke patients: focused review and results of new randomized controlled trial. J Rehabil Res Dev. 2011; 48: 355-66.

105. Masiero S, Celia A, Rosati G and Armani M. Robotic-assisted rehabilitation of the upper limb after acute stroke. Arch Phys Med Rehabil. 2007; 88: 142-9.

106. Treger I, Faran S and Ring H. Robot-assisted therapy for neuromuscular training of subacute stroke patients. A feasibility study. Eur J Phys Rehabil Med. 2008; 44: 431-5.

107. Bovolenta F, Sale P, Dall'Armi V, Clerici P and Franceschini M. Robot-aided therapy for upper limbs in patients with stroke-related lesions. Brief report of a clinical experience. J Neuroeng Rehabil. 2011; 8: 18.

108. Housman SJ, Scott KM and Reinkensmeyer DJ. A randomized controlled trial of gravitysupported, computer-enhanced arm exercise for individuals with severe hemiparesis. Neurorehabil Neural Repair. 2009; 23: 505-14.

109. Sanchez RJ, Liu J, Rao S, et al. Automating arm movement training following severe stroke: functional exercises with quantitative feedback in a gravity-reduced environment. IEEE Trans Neural Syst Rehabil Eng. 2006; 14: 378-89.

110. Reinkensmeyer DJ, Wolbrecht ET, Chan V, Chou C, Cramer SC and Bobrow JE. Comparison of three-dimensional, assist-as-needed robotic arm/hand movement training provided with Pneu-WREX to conventional tabletop therapy after chronic stroke. Am J Phys Med Rehabil. 2012; 91: S232-41.

111. Abdollahi F, Case Lazarro ED, Listenberger $M$, et al. Error augmentation enhancing arm recovery in individuals with chronic stroke: a randomized crossover design. Neurorehabil Neural Repair. 2014; 28: 120-8.

112. Byl NN, Abrams GM, Pitsch E, et al. Chronic stroke survivors achieve comparable outcomes following virtual task specific repetitive training guided by a wearable robotic orthosis (UL-EXO7) and actual task specific repetitive training guided by a physical therapist. J Hand Ther. 2013; 26: 343-52; quiz 52.

113. Rabin BA, Burdea GC, Roll DT, Hundal JS, Damiani F and Pollack S. Integrative rehabilitation of elderly stroke survivors: the design and evaluation of the BrightArm. Disabil Rehabil Assist Technol. 2012; 7: 323-35.

114. Dohle $\mathrm{Cl}$, Rykman A, Chang J and Volpe BT. Pilot study of a robotic protocol to treat shoulder subluxation in patients with chronic stroke. J Neuroeng Rehabil. 2013; 10: 88.

115. Frisoli A, Procopio C, Chisari C, et al. Positive effects of robotic exoskeleton training of upper limb reaching movements after stroke. J Neuroeng Rehabil. 2012; 9: 36.

116. Hu XL, Tong KY, Wei XJ, Rong W, Susanto EA and Ho SK. The effects of post-stroke upperlimb training with an electromyography (EMG)-driven hand robot. J Electromyogr Kinesiol. 2013; 23: 1065-74.

117. Merians AS, Tunik E, Fluet GG, Qiu Q and Adamovich SV. Innovative approaches to the rehabilitation of upper extremity hemiparesis using virtual environments. Eur J Phys Rehabil Med. 2009; 45: 123-33.

118. Emken JL, Benitez R, Sideris A, Bobrow JE and Reinkensmeyer DJ. Motor adaptation as a greedy optimization of error and effort. J Neurophysiol. 2007; 97: 3997-4006. 


\section{AdDitionAl FILE 1: SEARCH STRATEGY}

\section{Disorder}

1. "cerebrovascular disorders"[MeSH Terms]

2. CVA

3. stroke

4. "stroke"[MeSH Terms]

5. "hemiplegia"[MeSH Terms]

6. hemipleg*

7. hemipare*

8. cerebrovasc* OR cerebral vascular

9. \#1 OR \#2 OR \#3 OR \#4 OR \#5 OR \#6 OR \#7 OR \#8

\section{Intervention}

10. "robotics"[MeSH Terms]

11. robot*

12. \#10 OR \#11

\section{Body segment}

13. "upper extremity"[MeSH Terms]

14. "arm"[MeSH Terms]

15. upper extremity

16. upper extremities

17. arm

18. arms

19. upper limb

20. upper limbs

21. shoulder

22. elbow

23. wrist

24. hand

25. \#13 OR \#14 OR \#15 OR \#16 OR \#17 OR \#18 OR \#19 OR \#20 OR \#21 OR \#22 OR \#23 OR \#24

\section{Combination cerebrovascular disorders, robotics \& upper extremity}

26. \#9 AND \#12 AND \#25 
Chapter 2

\section{Limitations}

27. training

28. therapy

29. \#27 OR \#28

30. motor

31. function

32. \#30 OR \#31

\section{Total search strategy}

33. \#26 AND \#29 AND \#32 


\section{ADDITIONAL FILE 2: REVIEWED ARTICLES}

The spreadsheet on the next pages contains an entry for each of the groups, with all the aspects treated in this review (subjects group, arm segments, training modality and features of implementation and indicators for clinical outcome) and others as training duration, and the device used. The spreadsheet is also available online at:

https://jneuroengrehab.biomedcentral.com/articles/10.1186/1743-0003-11111\#MOESM2 
Chapter 2

Additional file 2: Reviewed articles

\begin{tabular}{|c|c|c|c|c|c|c|c|c|c|c|c|c|}
\hline Authors & Year & $\begin{array}{l}\text { Refe- } \\
\text { rence }\end{array}$ & $\begin{array}{l}\text { Study } \\
\text { design }\end{array}$ & $\begin{array}{c}\text { \# of } \\
\text { subjects }\end{array}$ & $\begin{array}{l}\text { Subject } \\
\text { type }\end{array}$ & $\begin{array}{c}\text { Arm } \\
\text { segments } \\
\text { involved }\end{array}$ & Device & $\begin{array}{c}\text { \# of } \\
\text { sessions } \\
\text { in total }\end{array}$ & $\begin{array}{l}\text { Session } \\
\text { duration } \\
\text { (min) }\end{array}$ & $\begin{array}{c}\text { \# of } \\
\text { weeks } \\
\text { training }\end{array}$ & $\begin{array}{l}\text { Therapy } \\
\text { duration (h) }\end{array}$ & $\begin{array}{l}\text { Task Uni-, } \\
\text { Bimanual }\end{array}$ \\
\hline Aisen et al. & 1997 & 45 & $\mathrm{RCT}$ & 10 & A & SE & MIT-MANUS & & & 6.4 & 28.80 & $\mathrm{U}$ \\
\hline Volpe et al. & 2000 & 46 & RCT & 30 & A & SE & MIT-MANUS & 25 & 60 & 5 & 25.00 & $\mathrm{U}$ \\
\hline Burgar et al. & 2000 & 70 & RCT & 11 & c & SE & MIME & 24 & 60 & 8 & 24.00 & B \\
\hline Lum et al. & 2002 & 71 & RCT & 13 & c & SE & MIME & 24 & 60 & 8 & 24.00 & B \\
\hline Ferraro et al. & 2003 & 52 & CT & 12 & c & SE & MIT-MANUS & 18 & 60 & 6 & 18.00 & $U$ \\
\hline Ferraro et al. & 2003 & 52 & & 16 & c & SE & MIT-MANUS & 18 & 60 & 6 & 18.00 & $\mathrm{U}$ \\
\hline Hesse et al. & 2003 & 65 & CT & 12 & c & FW & Bi-manu-track & 15 & 15 & 3 & 3.75 & B \\
\hline Fasoli et al. & 2004 & 43 & RCT & 30 & A & SE & MIT-MANUS & 25 & 60 & 5 & 25.00 & $\mathrm{U}$ \\
\hline Krebs et al. & 2004 & 50 & CT & 9 & c & SE & MIT-MANUS & 36 & 60 & 12 & 36.00 & u \\
\hline Stein et al. & 2004 & 51 & RCT & 9 & c & SE & InMotion2 & 18 & 60 & 6 & 18.00 & U \\
\hline Stein et al. & 2004 & 51 & & 9 & c & SE & InMotion2 & 18 & 60 & 6 & 18.00 & $U$ \\
\hline Stein et al. & 2004 & 51 & & 5 & c & SE & InMotion2 & 18 & 60 & 6 & 18.00 & $\mathrm{U}$ \\
\hline Stein et al. & 2004 & 51 & & 5 & c & SE & InMotion2 & 18 & 60 & 6 & 18.00 & $u$ \\
\hline Stein et al. & 2004 & 51 & & 18 & c & SE & InMotion2 & 18 & 60 & 6 & 18.00 & $\mathrm{U}$ \\
\hline Lum et al. & 2004 & 72 & CT & 6 & c & SE & MIME & 24 & 60 & 8 & 24.00 & $\mathrm{U}$ \\
\hline Lum et al. & 2004 & 72 & & 7 & c & SE & MIME & 24 & 60 & 8 & 24.00 & U \\
\hline Daly et al. & 2005 & 53 & RCT & 6 & c & SE & InMotion2 & 60 & 90 & 12 & 90.00 & U \\
\hline Macclellan et al. & 2005 & 54 & CT & 8 & c & SE & InMotion2 & 18 & 60 & 3 & 18.00 & $U$ \\
\hline Macclellan et al. & 2005 & 54 & & 19 & C & SE & InMotion2 & 18 & 60 & 3 & 18.00 & $\mathrm{U}$ \\
\hline Finley et al. & 2005 & 55 & CT & 15 & c & $\mathrm{SE}$ & InMotion2 & 18 & 60 & 3 & 18.00 & $U$ \\
\hline Hesse et al. & 2005 & 63 & RCT & 21 & A & FW & Bi-Manu-Track & 30 & 20 & 6 & 10.00 & B \\
\hline Lum et al. & 2006 & 69 & RCT & 5 & SA & SE & MIME & 15 & 60 & 4 & 15.00 & B \\
\hline Lum et al. & 2006 & 69 & & 9 & SA & SE & MIME & 15 & 60 & 4 & 15.00 & $\mathrm{U}$ \\
\hline Lum et al. & 2006 & 69 & & 10 & SA & SE & MIME & 15 & 60 & 4 & 15.00 & mixed \\
\hline Kahn et al. & 2006 & 81 & RCT & 10 & c & SE & ARM Guide & 24 & 45 & 8 & 18.00 & $U$ \\
\hline Sanchez et al. & 2006 & 109 & CT & 5 & c & SEWH & T-WREX & 24 & 45 & 8 & 18.00 & $U$ \\
\hline Colombo et al. & 2007 & 73 & RCT & 8 & C & w & $\begin{array}{l}1 \text { DoF robotic device for wrist } \\
\text { rehabilitation }\end{array}$ & 30 & 40 & 3 & 20.00 & $\mathrm{U}$ \\
\hline Colombo et al. & 2007 & 73 & & 12 & C & SE & $\begin{array}{l}2 \text { DoF robotic device for } \\
\text { elbow-shoulder rehabilitation }\end{array}$ & 30 & 40 & 3 & 20.00 & $\mathrm{U}$ \\
\hline Chang et al. & 2007 & 83 & CT & 20 & c & SE & $\begin{array}{l}\text { BFIAMT (bilateral force-indu- } \\
\text { ced isokinetic arm movement } \\
\text { trainer) }\end{array}$ & 24 & 30 & 8 & 12.00 & B \\
\hline Hu et al. & 2007 & 86 & CT & 7 & c & E & $\begin{array}{l}\text { electromyography-driven } \\
\text { motor system }\end{array}$ & 20 & 90 & 7 & 30.00 & $\mathrm{U}$ \\
\hline Stein et al. & 2007 & 90 & $\mathrm{CT}$ & 6 & C & $\mathrm{E}$ & AJB (Active Joint Brace) & 18 & 60 & $6-9$ & 18.00 & $\mathrm{U}$ \\
\hline $\begin{array}{l}\text { Amirabdollahian } \\
\text { et al. }\end{array}$ & 2007 & 94 & RCT & 15 & Mixed & SE & GENTLE/S & 9 & 30 & 3 & 4.50 & $U$ \\
\hline $\begin{array}{l}\text { Amirabdollahian } \\
\text { et al. }\end{array}$ & 2007 & 94 & & 16 & Mixed & SE & GENTLE/S & 27 & 30 & 9 & 13.50 & $u$ \\
\hline Fazekas et al. & 2007 & 103 & RCT & 15 & Mixed & SE & REHAROB & 20 & 30 & 4 & 10.00 & u \\
\hline
\end{tabular}




\begin{tabular}{|c|c|c|c|c|c|c|c|}
\hline $\begin{array}{l}\text { Outcomes on Body functions - } \\
\text { measured }\end{array}$ & $\begin{array}{l}\text { Outcomes on Body functions - } \\
\text { improved }\end{array}$ & $\begin{array}{l}\text { Outcomes on Activi- } \\
\text { ties - measured }\end{array}$ & $\begin{array}{l}\text { Outcomes on Acti- } \\
\text { vities - improved }\end{array}$ & $\begin{array}{l}\text { Outcomes on } \\
\text { Body functions - } \\
\text { improved }\end{array}$ & $\begin{array}{l}\text { Outcomes } \\
\text { on Activ. - } \\
\text { improved }\end{array}$ & $\begin{array}{l}\text { FM } \\
\text { base- } \\
\text { line }\end{array}$ & $\begin{array}{l}\text { FM std } \\
\text { if pro- } \\
\text { vided }\end{array}$ \\
\hline FM MSS MP & MSS & FIM & & 0 & 0 & 17.1 & 15.18 \\
\hline Fmsec FMwh MSSse MSSwh MP & MP MSSse & FIMmot FIMcog & FIMmot & 0 & 0 & 6 & 2.5 \\
\hline FMse FMwh strength reach & FMse strength reach & BI FIM & & 1 & 0 & 24.8 & 16.5 \\
\hline FMse FMhw strength reach & FMse strength reach & FIM BI & & 1 & 0 & 24.8 & 4.5 \\
\hline $\begin{array}{c}\text { FMsec FMhw MP MSSse MSSwh } \\
\text { MAS }\end{array}$ & FMsec MP & FIMupp FIMlow & FIMupp & 0 & 0 & 26.6 & $\begin{array}{l}\text { *sum } \\
\text { of w } \\
\text { and se }\end{array}$ \\
\hline $\begin{array}{c}\text { FMsec FMhw MP MSSse MSSwh } \\
\text { MAS }\end{array}$ & FMsec MP & FIMupp FIMlow & & 0 & 0 & 9.1 & $\begin{array}{l}\text { *sum } \\
\text { of w } \\
\text { and se }\end{array}$ \\
\hline MAS & MAS & RMA & & 1 & 0 & NA & \\
\hline FM MSSse MSSwh MRC & FM MSSse MRC & $\begin{array}{l}\text { FIMsc FIMmot } \\
\text { FIMcog }\end{array}$ & $\begin{array}{l}\text { FIMsc FIMmot } \\
\text { FIMcog }\end{array}$ & 1 & 1 & 9.6 & 0 \\
\hline Fmsec MSSse MP MAS & Fmsec MSSse MP MAS & & & 1 & NA & NA & Se only \\
\hline $\begin{array}{l}\text { FM MSSse MSSwh MRC MAS } \\
\text { MaxForce }\end{array}$ & FM MaxForce MSS & & & 0 & NA & 38 & 9.6 \\
\hline $\begin{array}{l}\text { FM MSSse MSSwh MRC MAS } \\
\text { MaxForce }\end{array}$ & FM MaxForce MSS & & & 0 & NA & 35.3 & 11.6 \\
\hline $\begin{array}{l}\text { FM MSSse MSSwh MRC MAS } \\
\text { MaxForce }\end{array}$ & FM MaxForce MSS & & & 0 & NA & 25.6 & 2.4 \\
\hline $\begin{array}{l}\text { FM MSSse MSSwh MRC MAS } \\
\text { MaxForce }\end{array}$ & FM MaxForce MSS & & & 0 & NA & 24.6 & 7.9 \\
\hline $\begin{array}{l}\text { FM MSSse MSSwh MRC MAS } \\
\text { MaxForce }\end{array}$ & FM MaxForce MSS & & & 0 & NA & 20.7 & 3.6 \\
\hline $\begin{array}{l}\text { WorkOutputPass WorkOutputAct } \\
\text { FDE speed smoothness }\end{array}$ & $\begin{array}{l}\text { WorkOutputAct FDE speed } \\
\text { smoothness }\end{array}$ & & & 1 & NA & NA & \\
\hline $\begin{array}{l}\text { WorkOutputPass WorkOutputAct } \\
\text { FDE ReachExtend }\end{array}$ & workOutputAct FDE ReachExtend & & & 1 & NA & NA & \\
\hline FM TargetAccuracy Smoothness & FM TargetAccuracy Smoothness & $\begin{array}{l}\text { AMAT AMATse } \\
\text { AMATwh }\end{array}$ & AMAT AMATse & 1 & 1 & 21 & 10 \\
\hline FMse FMwh FM MSSse MSSwh MP & & WMFTtime WMFTfa & WMFTfa & 0 & 0 & 26.8 & $\begin{array}{l}\text { *sum } \\
\text { of w } \\
\text { and se }\end{array}$ \\
\hline FMse FMwh FM MSSse MSSwh MP & FMse FM MP & WMFTtime WMFTfa & & 0 & 0 & 9 & \\
\hline $\begin{array}{l}\text { FM MP AimError MeanSpeed } \\
\text { PeakSpeed M:Pratio Duration }\end{array}$ & $\begin{array}{l}\text { FM MP AimError MeanSpeed } \\
\text { M:Pratio Duration }\end{array}$ & $\begin{array}{l}\text { WMFTtime WM- } \\
\text { FTscore }\end{array}$ & & 1 & 0 & 10.1 & 0.7 \\
\hline FM MRC MAS & FM MRC & & & 0 & NA & 7.3 & \\
\hline $\begin{array}{l}\text { FMse FMwh MSS MP MASprox } \\
\text { MASdis }\end{array}$ & & FIM & & 0 & 0 & 39.2 & $\begin{array}{l}\text { *sum } \\
\text { of w } \\
\text { and se }\end{array}$ \\
\hline $\begin{array}{l}\text { FMse FMwh MSS MP MASprox } \\
\text { MASdis }\end{array}$ & FMse FMwh MP & FIM & FIM & 0 & 1 & 31.6 & $\begin{array}{l}\text { *sum } \\
\text { of w } \\
\text { and se }\end{array}$ \\
\hline $\begin{array}{l}\text { FMse FMwh MSS MP MASprox } \\
\text { MASdis }\end{array}$ & FMse FMwh MP MSS & FIM & FIM & 0 & 1 & 21.7 & $\begin{array}{l}\text { *sum } \\
\text { of w } \\
\text { and se }\end{array}$ \\
\hline $\begin{array}{l}\text { FRs FSs Stiffness FRu Straightness } \\
\text { Smoothness Cmscore RLAFT }\end{array}$ & $\begin{array}{l}\text { FRs FSs Straightness Cmscore } \\
\text { RLAFT }\end{array}$ & & & 0 & NA & NA & \\
\hline $\begin{array}{l}\text { FM Rancho BBT BBTmod Grip- } \\
\text { Strength ROMsup ROMunsup }\end{array}$ & $\begin{array}{l}\text { FM GripStrength ROMsup } \\
\text { ROMunsup }\end{array}$ & & & 0 & NA & NA & \\
\hline FM MPS RS PI AMI & FM RS PI & & & 0 & NA & NA & $\begin{array}{l}\text { total } \\
\text { FM } \\
(/ 115)\end{array}$ \\
\hline FM MPS RS PI AMI VM MD nPL & FM MPS RS PI AMI VM MD nPL & & & 1 & NA & NA & $\begin{array}{l}\text { total } \\
\text { FM } \\
(/ 115)\end{array}$ \\
\hline $\begin{array}{l}\text { FM MAS GripStr PushStr PullStr } \\
\text { PeakVel \%TPV MTime NJS }\end{array}$ & $\begin{array}{l}\text { FM GripStr PushStr PullStr PeakVel } \\
\text { \%TPV MTime NJS }\end{array}$ & FAT & & 1 & 0 & 32.7 & 15.26 \\
\hline $\begin{array}{l}\text { MAS FM MSS RMSE EMGact- } \\
\text { Level Cl }\end{array}$ & $\begin{array}{l}\text { MAS FM MSS RMSE EMGact- } \\
\text { Level Cl }\end{array}$ & & & 1 & NA & 13.3 & 1.7 \\
\hline FM MAS MASe & FM MAS MASe & & & 1 & NA & 15.5 & 3.88 \\
\hline FM & $\mathrm{FM}$ & & & 1 & NA & NA & \\
\hline FM & FM & & & 1 & NA & NA & \\
\hline $\begin{array}{l}\text { RMA MASsa MASef FMse ROMs } \\
\text { ROMe }\end{array}$ & RMA MASsa MASef FMse ROMe & FIM & FIM & 1 & 1 & 18 & 25 \\
\hline
\end{tabular}


Chapter 2

Additional file 2: Reviewed articles (continued)

\begin{tabular}{|c|c|c|c|c|c|c|c|c|c|c|c|c|}
\hline Authors & Year & $\begin{array}{l}\text { Refe- } \\
\text { rence }\end{array}$ & $\begin{array}{l}\text { Study } \\
\text { design }\end{array}$ & $\begin{array}{c}\text { \# of } \\
\text { subjects }\end{array}$ & $\begin{array}{l}\text { Subject } \\
\text { type }\end{array}$ & $\begin{array}{c}\text { Arm } \\
\text { segments } \\
\text { involved }\end{array}$ & Device & $\begin{array}{c}\text { \# of } \\
\text { sessions } \\
\text { in total }\end{array}$ & $\begin{array}{c}\text { Session } \\
\text { duration } \\
\text { (min) }\end{array}$ & $\begin{array}{c}\text { \# of } \\
\text { weeks } \\
\text { training }\end{array}$ & $\begin{array}{l}\text { Therapy } \\
\text { duration }(\mathrm{h})\end{array}$ & $\begin{array}{l}\text { Task Uni-, } \\
\text { Bimanual }\end{array}$ \\
\hline Masiero et al. & 2007 & 105 & RCT & 17 & A & SEF & NeReBot & 50 & 24 & 5 & 20.00 & $\mathrm{U}$ \\
\hline Rabadi et al. & 2008 & 47 & RCT & 10 & A & SE & MIT-MANUS & 12 & 40 & $2-3$ & 8.00 & $\mathrm{U}$ \\
\hline Volpe et al. & 2008 & 56 & RCT & 11 & C & SE & InMotion2 & 18 & 60 & 6 & 18.00 & $\mathrm{U}$ \\
\hline Krebs et al. & 2008 & 49 & CT & 32 & C & SE & InMotion2 & 18 & 60 & 6 & 18.00 & 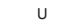 \\
\hline Krebs et al. & 2008 & 49 & & 10 & C & SEFWH & InMotion2 & 18 & 60 & 6 & 18.00 & $\mathrm{U}$ \\
\hline Krebs et al. & 2008 & 49 & & 5 & C & SEFWH & InMotion2 & 18 & 60 & 6 & 18.00 & $\mathrm{U}$ \\
\hline Song et al. & 2008 & 92 & CT & 8 & C & E & $\begin{array}{l}\text { myoelectrically-controlled } \\
\text { robotic system }\end{array}$ & 20 & 60 & 6 & 20.00 & $\mathrm{U}$ \\
\hline Takahashi et al. & 2008 & 96 & RCT & 6 & C & WH & HWARD & 15 & 90 & 3 & 22.50 & $\mathrm{U}$ \\
\hline Takahashi et al. & 2008 & 96 & & 7 & C & WH & HWARD & 15 & 90 & 3 & 22.50 & $\mathrm{U}$ \\
\hline Colombo et al. & 2008 & 99 & CT & 9 & A & SE & MEMOS & 30 & 32 & 3 & 16.00 & $\mathrm{U}$ \\
\hline Colombo et al. & 2008 & 99 & & 13 & C & SE & MEMOS & 30 & 32 & 3 & 16.00 & $U$ \\
\hline Treger et al. & 2008 & 106 & CT & 10 & A & SE & REOTM Therapy System & 15 & 45 & 3 & 11.25 & $\mathrm{U}$ \\
\hline Ellis et al. & 2009 & 80 & RCT & 7 & C & SE & $\begin{array}{l}\text { ACT3D (modified haptic master } \\
\text { robot) }\end{array}$ & 24 & $?$ & 8 & & $\mathrm{U}$ \\
\hline Ellis et al. & 2009 & 80 & & 7 & C & SE & $\begin{array}{l}\text { ACT3D (modified haptic master } \\
\text { robot) }\end{array}$ & 24 & $?$ & 8 & & $\mathrm{U}$ \\
\hline Cordo et al. & 2009 & 82 & CT & 5 & C & WH & AMES & 130 & 30 & 26 & 65.00 & $\mathrm{U}$ \\
\hline Casadio et al. & 2009 & 98 & CT & 10 & C & SE & Braccio di Ferro (BdF) & 10 & $60-75$ & 10 & 11.25 & $\mathrm{U}$ \\
\hline Hu et al. & 2009 & 88 & Ст & 15 & c & w & EMG-driven Motor System & 20 & 90 & 7 & 42.00 & $\mathrm{U}$ \\
\hline Housman et al. & 2009 & 108 & RCT & 14 & C & SEWH & T-WREX & 24 & 60 & 8 & 24.00 & $\mathrm{U}$ \\
\hline Posteraro et al. & 2010 & 57 & RCT & 17 & C & SE & MIT-MANUS & 18 & 45 & 6 & 13.50 & $u$ \\
\hline Posteraro et al. & 2010 & 57 & & 17 & C & SE & MIT-MANUS & 18 & 45 & 6 & 13.50 & $\mathrm{U}$ \\
\hline Lo et al. & 2010 & 27 & RCT & 49 & C & SEFWH & MIT-MANUS & 36 & 60 & 12 & 36.00 & $\mathrm{U}$ \\
\hline Kutner et al. & 2010 & 91 & RCT & 10 & Mixed & WH & Hand mentor robot system & & & 3 & 30.00 & $\mathrm{U}$ \\
\hline Colombo et al. & 2010 & 100 & CT & 18 & C & SE & MEMOS & 30 & 32 & 3 & 16.00 & $\mathrm{U}$ \\
\hline Conroy et al. & 2011 & 58 & RCT & 18 & C & SE & $\begin{array}{l}\text { InMotion } 2.0+\ln \text { Motion } \\
\text { Linear Robot }\end{array}$ & 18 & 60 & 6 & 18.00 & $\mathrm{U}$ \\
\hline Conroy et al. & 2011 & 58 & & 20 & c & SE & InMotion 2.0 & 18 & 60 & 6 & 18.00 & $\mathrm{U}$ \\
\hline Zollo et al. & 2011 & 60 & Ст & 24 & c & SEFW & Inmotion 2 and Inmotion 3 & 36 & 60 & 12 & 36.00 & $\mathrm{U}$ \\
\hline Hesse et al. & 2011 & 62 & RCT & 32 & A & FW & Bi-Manu-Track & 30 & 20 & 6 & 10.00 & B \\
\hline Hsieh et al. & 2011 & 64 & RCT & 6 & C & FW & Bi-Manu-Track & 20 & $90-105$ & 4 & 32.50 & B \\
\hline Hsieh et al. & 2011 & 64 & & 6 & C & FW & Bi-Manu-Track & 20 & $90-105$ & 4 & 32.50 & \\
\hline Burgar et al. & 2011 & 68 & RCT & 17 & A & SE & MIME & 30 & 60 & 3 & 15.80 & B \\
\hline Burgar et al. & 2011 & 68 & & 19 & A & SE & MIME & 15 & 60 & 3 & 8.60 & B \\
\hline Abdullah et al. & 2011 & 75 & RCT & 8 & A & SEW & 5DoF industrial robot & $24-33$ & 45 & $8-11$ & 21.38 & $\mathrm{U}$ \\
\hline Stein et al. & 2011 & 78 & CT & 12 & C & $\mathrm{H}$ & Amadeo & 18 & 60 & 6 & 18.00 & $\mathrm{U}$ \\
\hline Merians et al. & 2011 & 84 & CT & 12 & c & SEWH & $\begin{array}{l}\text { CyberGlove, CyberGrasp, Flock } \\
\text { of Birds, Haptic Master robot }\end{array}$ & 8 & $120-180$ & 2 & 22.00 & $\mathrm{u}$ \\
\hline
\end{tabular}




\begin{tabular}{|c|c|c|c|c|c|c|c|}
\hline $\begin{array}{l}\text { Outcomes on Body functions - } \\
\text { measured }\end{array}$ & $\begin{array}{l}\text { Outcomes on Body functions - } \\
\text { improved }\end{array}$ & $\begin{array}{l}\text { Outcomes on Activi- } \\
\text { ties - measured }\end{array}$ & $\begin{array}{l}\text { Outcomes on Acti- } \\
\text { vities - improved }\end{array}$ & $\begin{array}{l}\text { Outcomes on } \\
\text { Body functions - } \\
\text { improved }\end{array}$ & $\begin{array}{l}\text { Outcomes } \\
\text { on Activ. - } \\
\text { improved }\end{array}$ & $\begin{array}{l}\mathrm{FM} \\
\text { base- } \\
\text { line }\end{array}$ & $\begin{array}{l}\text { FM std } \\
\text { if pro- } \\
\text { vided }\end{array}$ \\
\hline $\begin{array}{l}\text { FMse FMwh MRCdel MRCbic } \\
\text { MRCwf TCT MAS }\end{array}$ & $\begin{array}{l}\text { FMse MRCdel MRCbic MRCwf } \\
\text { FIM FIMmot }\end{array}$ & FIM FIMmot & & 1 & 0 & 8 & \\
\hline $\begin{array}{c}\text { FMse FMwh MSSse MSSwh MPS } \\
\text { MAS pain }\end{array}$ & & $\begin{array}{l}\text { FIM FIMcog FIMmot } \\
\text { ARAT }\end{array}$ & FIM FIMmot ARAT & 0 & 1 & 8 & $\begin{array}{l}{ }^{*} \text { sum } \\
\text { of w } \\
\text { and se }\end{array}$ \\
\hline FMse FMwh MP MAS Jtstab Pain & Fmse MP & ARAT & & 0 & 0 & 15.24 & $\begin{array}{l}\text { *sum } \\
\text { of w } \\
\text { and se }\end{array}$ \\
\hline FM & FM & & & 1 & NA & 25 & 9.6 \\
\hline FM & FM & & & 1 & NA & 30.7 & 16.3 \\
\hline FM & FM & & & 1 & NA & 21.4 & 4.9 \\
\hline $\begin{array}{l}\text { FMse FMwh MSS MAS ExtRange } \\
\text { MIVF MIVE RMSE }\end{array}$ & $\begin{array}{l}\text { FMse MSS MAS ExtRange MIVF } \\
\text { MIVE RMSE }\end{array}$ & & & 1 & NA & 19.13 & $\begin{array}{l}\text { *sum } \\
\text { of w } \\
\text { and se }\end{array}$ \\
\hline $\begin{array}{c}\text { BBT FM FMhw Fmse MASw MASe } \\
\text { ROMwe NHPT GraspF PinchF } \\
\text { EMGactivity }\end{array}$ & BBT FM FMhw & ARAT & ARAT & 0 & 1 & 49.5 & 8.6 \\
\hline $\begin{array}{c}\text { BBT FM FMhw Fmse MASw MASe } \\
\text { ROMwe NHPT GraspF PinchF } \\
\text { EMGactivity }\end{array}$ & $\begin{array}{l}\text { BBT FM FMhw Fmse MASe ROMwe } \\
\text { SIS GraspF PinchF }\end{array}$ & ARAT & ARAT & 1 & 1 & 40.4 & 10.5 \\
\hline $\begin{array}{l}\text { MPS MSS FM AMI VM MD nPL } \\
\text { nPeaks Jerk nFCP }\end{array}$ & $\begin{array}{l}\text { MPS MSS FM AMI VM nPL } \\
\text { nPeaks Jerk }\end{array}$ & & & 1 & NA & NA & $\begin{array}{l}\text { total } \\
\text { FM } \\
(/ 115)\end{array}$ \\
\hline $\begin{array}{l}\text { MPS MSS FM AMI VM MD nPL } \\
\text { nPeaks Jerk nFCP }\end{array}$ & $\begin{array}{l}\text { MPS MSS FM AMI VM MD } \\
\text { nPeaks Jerk }\end{array}$ & & & 1 & NA & NA & $\begin{array}{c}\text { total } \\
\text { FM } \\
(/ 115)\end{array}$ \\
\hline FM MFTprox MFTdist MFT & FM MFTprox MFTdist MFT & & & 1 & NA & 35 & \\
\hline WorkArea Strength MVT & WorkArea & & & 0 & NA & NA & \\
\hline WorkArea Strength MVT & WorkArea & & & 0 & NA & NA & \\
\hline Strength JointPos & & & & 0 & NA & NA & \\
\hline FM MAS I1 I2 I3 I4 & FM I1 I2 I3 I4 & & & 1 & NA & 15 & 13 \\
\hline FMse FMwh MASe MASw & Fmse FMwh MASe MASw & ARAT FIM & & 1 & 0 & 30 & $\begin{array}{l}\text { approx } \\
\text { from } \\
\text { graph }\end{array}$ \\
\hline $\begin{array}{l}\text { FM RFTlevel RFTspeed Strength } \\
\text { ROM }\end{array}$ & FM ROM & MALqom MALaou & & 0 & 0 & 21.7 & 5.9 \\
\hline $\begin{array}{l}\text { MSSse MASs MASe ROMs pROMe } \\
\text { ACTee } \triangle \text { MAS }\end{array}$ & MSSse MASs ROMs pROMe & & & 0 & NA & NA & \\
\hline $\begin{array}{c}\text { MSSse MASs MASe ROMs pROMe } \\
\text { ACTee } \triangle \text { MAS }\end{array}$ & MSSse MASs ROMs ACTee & & & 0 & NA & NA & \\
\hline \multirow[t]{2}{*}{ FMA PAIN MAS } & & WMFT & & 0 & 0 & 19.7 & 10.7 \\
\hline & & & & 1 & NA & NA & \\
\hline MSS FM nFCP FDE & MSS FM nFCP FDE & & & 1 & NA & 21.44 & 8.58 \\
\hline $\begin{array}{l}\text { FMA Aim Dev Mspeed Pspeed } \\
\text { Smoothness Duration ERatio Force }\end{array}$ & FMA Aim & WMFT & & 0 & 0 & 20.3 & \\
\hline $\begin{array}{l}\text { FMA Aim Dev Mspeed Pspeed } \\
\text { Smoothness Duration ERatio Force }\end{array}$ & FMA Aim & WMFT & WMFT & 0 & 1 & 16.5 & \\
\hline $\begin{array}{c}\text { FM MP UMTaimangle UMTlength } \\
\text { UMTjerk UMTforce UMTwork RM- } \\
\text { Taimangle RMTlength RMTforce } \\
\text { RMTwork }\end{array}$ & $\begin{array}{l}\text { FM MP UMTaimangle UMTlength } \\
\text { UMTjerk UMTforce UMTwork } \\
\text { RMTaimangle RMTlength RMTforce } \\
\text { RMTwork }\end{array}$ & & & 1 & NA & 22.26 & 9.841 \\
\hline FM MRC MAS BBT & $\mathrm{FM}$ & $\mathrm{BI}$ & & 0 & 0 & 8.2 & 4.4 \\
\hline FM MRC & FM & $\begin{array}{l}\text { MALaou MALqom } \\
\text { ABILHAND }\end{array}$ & MALqom & 1 & 0 & 44 & 8.17 \\
\hline FM MRC & FM & $\begin{array}{l}\text { MALaou MALqom } \\
\text { ABILHAND }\end{array}$ & MALqom & 1 & 0 & 37.67 & 10 \\
\hline FM Fmse MP MAS & & $\begin{array}{l}\text { FIM WMFTfas } \\
\text { WMFTmt }\end{array}$ & FIM & 0 & 0 & 19 & 3.7 \\
\hline FM Fmse MP MAS & - & $\begin{array}{l}\text { FIM WMFTfas } \\
\text { WMFTmt }\end{array}$ & & 0 & 0 & 26.7 & 5 \\
\hline CMSA & CMSA & CAHAI-7 & CAHAI-7 & 1 & 1 & NA & \\
\hline $\begin{array}{c}\text { FM 9HPT aROM1 aROM2 aROM3 } \\
\text { aROM4 aROM5 }\end{array}$ & FM aROM1 aROM2 & $\begin{array}{l}\text { MALaou MALqou } \\
\text { MAM36 JHFT }\end{array}$ & $\begin{array}{l}\text { MALaou MALqou } \\
\text { MAM36 JHFT }\end{array}$ & 0 & 1 & 37.9 & 11.1 \\
\hline $\begin{array}{c}\text { Fractionation TimeVPT Acc TimeHT } \\
\text { PathLength Smoothness Deviation } \\
\text { MPJext }\end{array}$ & $\begin{array}{l}\text { Fractionation TimeVPT TimeHT } \\
\text { PathLength Smoothness Deviation }\end{array}$ & WMFT JTHF & WMFT JTHF & 1 & 1 & NA & \\
\hline
\end{tabular}




\section{Chapter 2}

Additional file 2: Reviewed articles (continued)

\begin{tabular}{|c|c|c|c|c|c|c|c|c|c|c|c|c|}
\hline Authors & Year & $\begin{array}{l}\text { Refe- } \\
\text { rence }\end{array}$ & $\begin{array}{l}\text { Study } \\
\text { design }\end{array}$ & $\begin{array}{c}\text { \# of } \\
\text { subjects }\end{array}$ & $\begin{array}{l}\text { Subject } \\
\text { type }\end{array}$ & $\begin{array}{c}\text { Arm } \\
\text { segments } \\
\text { involved }\end{array}$ & Device & $\begin{array}{c}\text { \# of } \\
\text { sessions } \\
\text { in total }\end{array}$ & $\begin{array}{c}\text { Session } \\
\text { duration } \\
\text { (min) }\end{array}$ & $\begin{array}{c}\text { \# of } \\
\text { weeks } \\
\text { training }\end{array}$ & $\begin{array}{c}\text { Therapy } \\
\text { duration (h) }\end{array}$ & $\begin{array}{l}\text { Task Uni-, } \\
\text { Bimanual }\end{array}$ \\
\hline Wei et al. & 2011 & 85 & RCT & 27 & C & W & PolyJbot & 20 & & $4-7$ & & U \\
\hline Wei et al. & 2011 & 85 & & & C & W & CYBEX and NORM & 20 & & $4-7$ & & U \\
\hline Lambercy et al. & 2011 & 95 & CT & 13 & c & FWH & HapticKnob & 18 & 60 & 6 & 18.00 & $\mathrm{U}$ \\
\hline Riley et al. & 2011 & 97 & СT & 23 & c & WH & HWARD & & & & 23.50 & $\mathrm{U}$ \\
\hline Masiero et al. & 2011 & 104 & RCT & 11 & A & SEF & NeReBot & 50 & 20 & 5 & 16.67 & $\mathrm{U}$ \\
\hline Bovolenta et al. & 2011 & 107 & CT & 19 & c & SE & ReoGo ${ }^{\mathrm{TM}}$ system & 20 & 45 & 4 & 15.00 & $\mathrm{U}$ \\
\hline Dipietro et al. & 2012 & 48 & СT & 42 & A & $\mathrm{SE}$ & MIT-MANUS, InMotion 2 & 18 & 60 & & 18.00 & u \\
\hline
\end{tabular}

\begin{tabular}{llllllllllll}
\hline Dipietro et al. & 2012 & 48 & 116 & C & SE & MIT-MANUS, InMotion2 & 18 & 60 & U
\end{tabular}

\begin{tabular}{|c|c|c|c|c|c|c|c|c|c|c|c|c|}
\hline Liao et al. & 2012 & 66 & RCT & 10 & c & FW & Bi-Manu-Track & 20 & $90-105$ & 4 & 32.50 & B \\
\hline Hwang et al. & 2012 & 79 & RCT & 6 & Mixed & $\mathrm{H}$ & Amadeo & 20 & 45 & 4 & 15.00 & $u$ \\
\hline Hwang et al. & 2012 & 79 & & 9 & Mixed & $\mathrm{H}$ & Amadeo & 20 & 45 & 4 & 15.00 & $u$ \\
\hline Duret et al. & 2013 & 44 & СT & 10 & A & SE & InMotion2 & $54( \pm 13)$ & 45 & $\begin{array}{c}15 \\
( \pm 3.5)\end{array}$ & 40.50 & $u$ \\
\hline Song et al. & 2013 & 93 & СT & 6 & c & E & $\begin{array}{l}\text { Myoelectrically-controlled } \\
\text { robotic system }\end{array}$ & 20 & 36 & 4 & 12.00 & \\
\hline Byl et al. & 2013 & 112 & RCT & 5 & c & SEFW & UL-EX07 robotic orthosis & 12 & 90 & 6 & 18.00 & $\mathrm{u}$ \\
\hline Byl et al. & 2013 & 112 & & 5 & c & SEFW & UL-EX07 robotic orthosis & 12 & 90 & 6 & 18.00 & B \\
\hline $\begin{array}{l}\text { Reinkensmeyer } \\
\text { et al. }\end{array}$ & 2012 & 110 & RCT & 13 & c & SEH & Pneu-WREX & 24 & 60 & 8 & 24.00 & U \\
\hline Wu et al. & 2012 & 67 & RCT & 14 & c & FW & Bi-Manu-Track & 20 & $90-105$ & 4 & 32.50 & B \\
\hline
\end{tabular}

\begin{tabular}{|c|c|c|c|c|c|c|c|c|c|c|c|c|}
\hline Mazzoleni et al. & 2013 & 59 & СT & 32 & c & SE & InMotion 2 & 20 & $?$ & 4 & & $u$ \\
\hline Mazzoleni et al. & 2013 & 59 & & 32 & c & SEFW & InMotion 3 & 40 & ? & 4 & & $\mathrm{u}$ \\
\hline Abdollahi et al. & 2013 & 111 & RCT & 26 & c & SE & VRROOM, PHANTOM, WREX & 12 & 60 & 4 & 12.00 & $\mathrm{U}$ \\
\hline Pinter et al. & 2013 & 76 & CT & 7 & A & $\mathrm{H}$ & Amadeo & 15 & 20 & 3 & 5.00 & $\mathrm{u}$ \\
\hline Sale et al. & 2012 & 77 & СT & 7 & A & H & Amadeo & 20 & 40 & 4 & 13.33 & $u$ \\
\hline Rabin et al. & 2012 & 113 & СT & 5 & c & SEWH & BrightArm & 18 & $20-50$ & 6 & 12.00 & $u$ \\
\hline Pellegrino et al. & 2012 & 61 & СT & 7 & c & SEFW & InMotion2, InMotion3 & 36 & 60 & 12 & 36.00 & $\mathrm{u}$ \\
\hline Song et al. & 2013 & 87 & CT & 16 & c & W & EMG driven exoskeleton & 20 & & $5-7$ & $?$ & $\mathrm{u}$ \\
\hline
\end{tabular}




\begin{tabular}{|c|c|c|c|c|c|c|c|}
\hline $\begin{array}{l}\text { Outcomes on Body functions - } \\
\text { measured }\end{array}$ & $\begin{array}{l}\text { Outcomes on Body functions - } \\
\text { improved }\end{array}$ & $\begin{array}{l}\text { Outcomes on Activi- } \\
\text { ties - measured }\end{array}$ & $\begin{array}{l}\text { Outcomes on Acti- } \\
\text { vities - improved }\end{array}$ & $\begin{array}{l}\text { Outcomes on } \\
\text { Body functions - } \\
\text { improved }\end{array}$ & $\begin{array}{l}\text { Outcomes } \\
\text { on Activ. - } \\
\text { improved }\end{array}$ & $\begin{array}{l}\text { FM } \\
\text { base- } \\
\text { line }\end{array}$ & $\begin{array}{l}\text { FM std } \\
\text { if pro- } \\
\text { vided }\end{array}$ \\
\hline $\begin{array}{l}\text { FM FMse FMwh MSS MSSwh } \\
\text { MSSse MASe MASw }\end{array}$ & $\begin{array}{c}\text { FM FMse FMwh MSS MSSwh } \\
\text { MASe MASw }\end{array}$ & ARAT FIM & & 1 & 0 & 29.93 & 7.66 \\
\hline $\begin{array}{l}\text { FM FMse FMwh MSS MSSwh } \\
\text { MSSse MASe MASw }\end{array}$ & FM FMse MSS MASw & ARAT FIM & & 0 & 0 & 27.5 & 9.54 \\
\hline $\begin{array}{c}\text { FM FMwh FMse MI MIhf Mlse } \\
\text { MAS FTHUE GripForce VAS Ep } \\
\text { NO Tr Ta }\end{array}$ & FM FMwh MI Mlse Ep NO Tr Ta & MotorAS & MotorAS & 0 & 1 & 32.15 & 11.31 \\
\hline FM B\&B & FM B\&B & ARAT & ARAT & 1 & 1 & 37.9 & 3.1 \\
\hline $\begin{array}{l}\text { MRCd MRCb MRCt MRCwf MRCwe } \\
\text { FM Fmse FMwh BBT MAS }\end{array}$ & $\begin{array}{l}\text { MRCd MRCb MRCt MRCwf MRCwe } \\
\text { FM Fmse FMwh BBT MAS }\end{array}$ & FIM FAT & FIM FAT & 1 & 1 & 30.5 & \\
\hline FM MRC AS VASpain BBT & FM MRC AS BBT & FAT FIM TUG & FAT FIM TUG & 1 & 1 & 31.21 & 16.92 \\
\hline $\begin{array}{l}\text { FM MeanSpeed PeakSpeed } \\
\text { Duration SpeedShape \#Peaks Jerk } \\
\text { \#Submovements SubmPeak Sub- } \\
\text { mDuration SubmOverlap SubmID } \\
\text { SubmSkewness SubmKurtosis } \\
\text { MeanSpeed PeakSpeed Duration } \\
\text { SpeedShape \#Peaks Jerk AxesRatio } \\
\text { \#Submovements SubmPeak Sub- } \\
\text { mDuration SubmOverlap SubmID } \\
\text { SubmSkewness SubmKurtosis }\end{array}$ & $\begin{array}{c}\text { FM MeanSpeed PeakSpeed } \\
\text { Duration SpeedShape \#Peaks Jerk } \\
\text { \#Submovements SubmPeak Sub- } \\
\text { mOverlap SubmID SubmKurtosis } \\
\text { MeanSpeed PeakSpeed Duration } \\
\text { SpeedShape \#Peaks AxesRatio } \\
\text { SubmPeak SubmDuration SubmO- } \\
\text { verlap SubmKurtosis }\end{array}$ & & & 1 & NA & 10.02 & 1.14 \\
\hline $\begin{array}{l}\text { FM MeanSpeed PeakSpeed } \\
\text { Duration SpeedShape \#Peaks Jerk } \\
\text { \#Submovements SubmPeak Sub- } \\
\text { mDuration SubmOverlap SubmID } \\
\text { SubmSkewness SubmKurtosis } \\
\text { MeanSpeed PeakSpeed Duration } \\
\text { SpeedShape \#Peaks Jerk AxesRatio } \\
\text { \#Submovements SubmPeak Sub- } \\
\text { mDuration SubmOverlap SubmID } \\
\text { SubmSkewness SubmKurtosis }\end{array}$ & $\begin{array}{l}\text { FM MeanSpeed Duration SpeedS- } \\
\text { hape \#Peaks Jerk \#Submove- } \\
\text { ments SubmPeak SubmDuration } \\
\text { SubmOverlap SubmKurtosis } \\
\text { SpeedShape \#Peaks Jerk AxesRatio } \\
\text { \#Submovements SubmPeak Sub- } \\
\text { mDuration SubmOverlap SubmID } \\
\text { SubmKurtosis }\end{array}$ & & & 1 & NA & 20.47 & 1.15 \\
\hline ArmActivityRatio FM & ArmActivityRatio FM & $\begin{array}{l}\text { FIM MALaou MAL- } \\
\text { qom ABILHAND }\end{array}$ & $\begin{array}{l}\text { MALaou MALqom } \\
\text { ABILHAND }\end{array}$ & 1 & 1 & 44.9 & 9.02 \\
\hline $\begin{array}{l}\text { FMwh FMse AS 9HPT GraspForce } \\
\text { PinchForce aROM-MCP2 }\end{array}$ & $\begin{array}{c}\text { FMwh GraspForce PinchForce } \\
\text { aROM-MCP2 }\end{array}$ & JTT & JTT & 0 & 1 & 39 & $\begin{array}{l}\text { *sum } \\
\text { of w } \\
\text { and se }\end{array}$ \\
\hline $\begin{array}{l}\text { FMwh FMse AS 9HPT GraspForce } \\
\text { PinchForce aROM-MCP2 }\end{array}$ & $\begin{array}{c}\text { FMwh GraspForce PinchForce } \\
\text { aROM-MCP2 }\end{array}$ & JTT & JTT & 0 & 1 & 40.5 & $\begin{array}{l}{ }^{*} \text { sum } \\
\text { of w } \\
\text { and se }\end{array}$ \\
\hline $\begin{array}{l}\text { FM, FMse, FMw, FMh, FMc, MSS, } \\
\text { MSSs, MSSe, MSSw, MSSh, \#move- } \\
\text { ments efficacy, RMSerror, velocity, } \\
\text { \#traj.reversals }\end{array}$ & $\begin{array}{l}\text { FM, FMse, FMh, MSS, MSSs, MSSe, } \\
\text { MSSw, \#movements efficacy, } \\
\text { velocity, \#traj.reversals }\end{array}$ & & & 1 & 0 & 14.9 & 5.8 \\
\hline $\begin{array}{c}\text { FMse, FMwh, MAS, RMSE, RMSJ, } \\
\text { ROM, Cl }\end{array}$ & FMse, ROM, RMSE, RMSJ, Cl & & & 1 & 0 & 18.8 & $\begin{array}{l}\text { *sum } \\
\text { of w } \\
\text { and se }\end{array}$ \\
\hline $\begin{array}{l}\text { FM, ROM, strength, pain, MSPS_ } \\
\text { (BBT_tapper), MAS }\end{array}$ & FM, ROM & MPS_(WMFT_DRTT) & & 1 & 0 & 23.8 & 7.7 \\
\hline $\begin{array}{l}\text { FM, ROM, strength, pain, MSPS_ } \\
\text { (BBT_tapper), MAS }\end{array}$ & FM, ROM & MPS_(WMFT_DRTT) & & 0 & 0 & 24.4 & 5.2 \\
\hline FM, BBT, GRIP, NSA & FM, BBT, GRIP, NSA & $\begin{array}{l}\text { RFT, MAL_AOU, } \\
\text { MAL_QOM }\end{array}$ & MAL_QOM & 1 & 0 & 24.6 & 9.1 \\
\hline $\begin{array}{l}\text { NMT_U, NMU_U, NTD_U, } \\
\text { SlopeSt_U, SlopeMid_U, ShFI_U, } \\
\text { ElEx_U, NMT_B, NMU_B, NTD_B, } \\
\text { SlopeSt_B, SlopeMid_B, ShFI_B, } \\
\text { ElEx_B,FMtot, FMprox, Fmdis }\end{array}$ & ShFI_U, ShFI_B & MALaou, MALqom & - & 0 & 0 & 43.29 & 10.09 \\
\hline FMse, MI, Velocity, MD & FMse, MI, Velocity, MD & & & 1 & NA & NA & NA \\
\hline FMse, FMw, MI, Velocity, MD & FMse, FMw, MI, Velocity, MD & & & 1 & NA & 31.28 & $\begin{array}{l}\text { *sum } \\
\text { of } w \\
\text { and se }\end{array}$ \\
\hline FM, BBT, ROM & $\mathrm{FM}$ & WMFT & WMFT & 0 & 1 & NA & \\
\hline MI, Mipinch, NIHSS, GripStrength & MI, Mipinch, GripStrength & $\mathrm{RMI}, \mathrm{mRS}, \mathrm{BI}$ & & 1 & 0 & NA & NA \\
\hline $\begin{array}{l}\text { FM, MRCwrist, MRChand, MI, AS, } \\
\text { Strength }\end{array}$ & MRCwrist, MRChand & BI, FIM, COPM & & 0 & 0 & 47.4 & 22.77 \\
\hline $\begin{array}{l}\text { StrengthShoulder, StrengthGrasp, } \\
\text { StrengthPinch, aROMSh, aROMwr, } \\
\text { FM, ADLindependence, BDI-II }\end{array}$ & $\begin{array}{l}\text { StrengthShoulder, StrengthGrasp, } \\
\text { aROMSh }\end{array}$ & $\begin{array}{l}\text { JHFT, ADLindepen- } \\
\text { dence }\end{array}$ & & 0 & 0 & 11.6 & NA \\
\hline $\begin{array}{l}\text { FM, MVC, ContractionLevel, } \\
\text { ContractionQuality }\end{array}$ & $\begin{array}{l}\text { FM, MVC, ContractionLevel, } \\
\text { ContractionQuality }\end{array}$ & & & 1 & NA & 31.8 & $\begin{array}{l}\text { *sum } \\
\text { of w } \\
\text { and se }\end{array}$ \\
\hline $\begin{array}{l}\text { FM, FMse, FMwh, MAS, RMSE, } \\
\text { ROM, MIVF, MIVE }\end{array}$ & $\begin{array}{c}\text { FM, FMse, FMwh, MAS, RMSE, } \\
\text { MIVF, MIVE }\end{array}$ & & & 1 & NA & 29.4 & 7.7 \\
\hline
\end{tabular}


Chapter 2

Additional file 2: Reviewed articles (continued)

\begin{tabular}{|c|c|c|c|c|c|c|c|c|c|c|c|c|}
\hline Authors & Year & $\begin{array}{l}\text { Refe- } \\
\text { rence }\end{array}$ & $\begin{array}{l}\text { Study } \\
\text { design }\end{array}$ & $\begin{array}{c}\text { \# of } \\
\text { subjects }\end{array}$ & $\begin{array}{l}\text { Subject } \\
\text { type }\end{array}$ & $\begin{array}{c}\text { Arm } \\
\text { segments } \\
\text { involved }\end{array}$ & Device & $\begin{array}{c}\text { \# of } \\
\text { sessions } \\
\text { in total }\end{array}$ & $\begin{array}{c}\text { Session } \\
\text { duration } \\
\text { (min) }\end{array}$ & $\begin{array}{c}\text { \# of } \\
\text { weeks } \\
\text { training }\end{array}$ & $\begin{array}{c}\text { Therapy } \\
\text { duration (h) }\end{array}$ & $\begin{array}{l}\text { Task Uni-, } \\
\text { Bimanual }\end{array}$ \\
\hline Dohle et al. & 2013 & 114 & CT & 18 & c & s & Linear shoulder robot & 18 & & 6 & & $\mathrm{U}$ \\
\hline Frisoli et al. & 2012 & 115 & CT & 9 & c & SEF & L-Exos & 18 & 60 & 6 & 18.00 & $u$ \\
\hline Colombo et al. & 2013 & 102 & CT & 20 & SA & SE & MEMOS, Braccio di Ferro & 30 & 30 & 3 & 15.00 & $\mathrm{u}$ \\
\hline Colombo et al. & 2013 & 102 & & 21 & c & SE & MEMOS, Braccio di Ferro & 30 & 30 & 3 & 15.00 & $\mathrm{U}$ \\
\hline Hu et al. & 2013 & 116 & CT & 10 & c & $\mathrm{H}$ & EMG-driven hand robot & 20 & 60 & 7 & $?$ & $\mathrm{U}$ \\
\hline Panarese et al. & 2012 & 101 & CT & 18 & c & SE & MEMOS & 30 & $?$ & 3 & $?$ & $\mathrm{U}$ \\
\hline Squeri et al. & 2013 & 74 & СT & 9 & c & FW & 3DoF wrist robotic exoskeleton & 10 & 60 & 2 & 10.00 & u \\
\hline
\end{tabular}

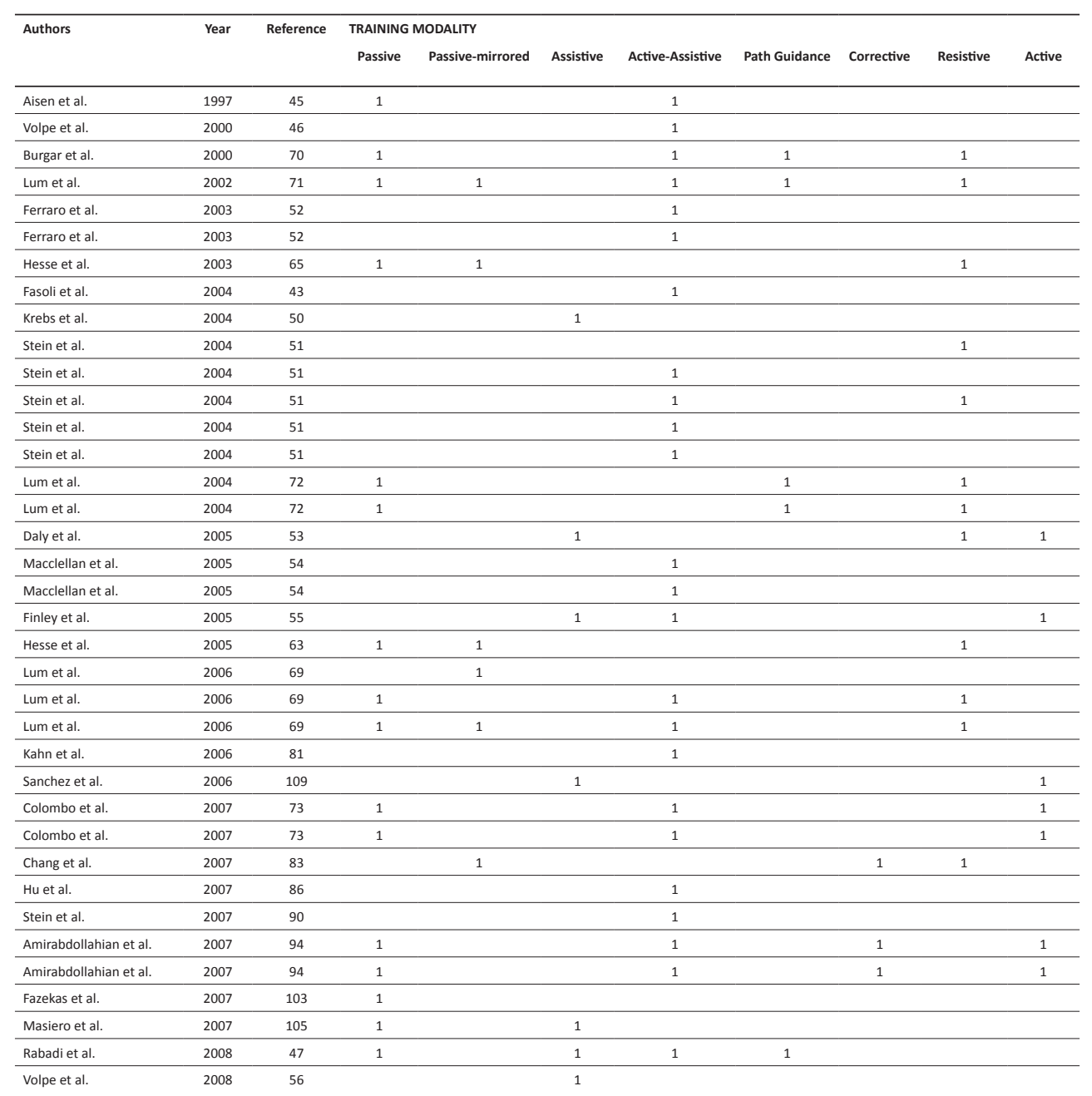




\begin{tabular}{|c|c|c|c|c|c|c|c|}
\hline $\begin{array}{l}\text { Outcomes on Body functions - } \\
\text { measured }\end{array}$ & $\begin{array}{l}\text { Outcomes on Body functions - } \\
\text { improved }\end{array}$ & $\begin{array}{l}\text { Outcomes on Activi- } \\
\text { ties - measured }\end{array}$ & $\begin{array}{l}\text { Outcomes on Acti- } \\
\text { vities - improved }\end{array}$ & $\begin{array}{l}\text { Outcomes on } \\
\text { Body functions - } \\
\text { improved }\end{array}$ & $\begin{array}{l}\text { Outcomes } \\
\text { on Activ. - } \\
\text { improved }\end{array}$ & $\begin{array}{l}\text { FM } \\
\text { base- } \\
\text { line }\end{array}$ & $\begin{array}{l}\mathrm{FM} \text { std } \\
\text { if pro- } \\
\text { vided }\end{array}$ \\
\hline $\begin{array}{c}\text { StabilitySh, MAS, FMse, FMwh } \\
\text { MotorPowerScapular, MotorPo- } \\
\text { werRotatorCuff }\end{array}$ & $\begin{array}{c}\text { StabilitySh, MAS, FMse, Motor- } \\
\text { PowerScapular, MotorPowerRo- } \\
\text { tatorCuff }\end{array}$ & & & 1 & NA & 13.6 & 1.2 \\
\hline $\begin{array}{c}\text { FM, MAS, aROMsh, aROMel, MT, } \\
\text { Smoothness, CCI_AD/PD, CCI_TB/ } \\
\text { BP, el-sh_excersion }\end{array}$ & $\begin{array}{l}\text { FM, MAS, aROMsh, aROMel, MT, } \\
\text { Smoothness, CCI_AD/PD }\end{array}$ & & & 1 & NA & 35.8 & 18.2 \\
\hline $\begin{array}{c}\text { FM, MASsh, MASel, AMI, MV, } \\
\text { nPL, SM }\end{array}$ & $\mathrm{FM}, \mathrm{AMI}, \mathrm{MV}, \mathrm{nPL}, \mathrm{SM}$ & & & 1 & NA & 24.6 & 11.2 \\
\hline $\begin{array}{c}\text { FM, MASsh, MASel, AMI, MV, } \\
\text { nPL, SM }\end{array}$ & $\mathrm{FM}, \mathrm{AMI}, \mathrm{MV}, \mathrm{nPL}, \mathrm{SM}$ & & & 1 & NA & 20 & 8.2 \\
\hline $\begin{array}{l}\text { FMse, FMwh, MASwr, MASfi, } \\
\text { MASel, EMG }\end{array}$ & FMse, FMwh, MASfi, EMG & $\begin{array}{l}\text { ARAT, WMFTscore, } \\
\text { WMFTtime }\end{array}$ & $\begin{array}{l}\text { ARAT, WMFTscore, } \\
\text { WMFTtime }\end{array}$ & 1 & 1 & 28 & 7.9 \\
\hline \%Succ, MV, nMD, nPK & $\%$ Succ, MV, nMD, nPK & & & 1 & 1 & NA & NA \\
\hline ROM, RoAM, jerk, FM & ROM, FM & WMFT & WMFT & 0 & 1 & 21 & 3.17 \\
\hline
\end{tabular}

\begin{tabular}{|c|c|c|c|c|c|c|c|c|c|c|c|}
\hline \multicolumn{12}{|c|}{ FEATURE OF HRI } \\
\hline Passive & $\begin{array}{l}\text { Passive- } \\
\text { mirrored }\end{array}$ & $\begin{array}{l}\text { Spring against } \\
\text { movement }\end{array}$ & $\begin{array}{l}\text { Moving } \\
\text { attractor }\end{array}$ & $\begin{array}{l}\text { Triggered } \\
\text { assistance }\end{array}$ & $\begin{array}{l}\text { Pushing force (in } \\
\text { case of delay) }\end{array}$ & $\begin{array}{l}\text { Emg-pro- } \\
\text { portional }\end{array}$ & Tunnels & $\begin{array}{l}\text { Spring-damper } \\
\text { guidance }\end{array}$ & $\begin{array}{l}\text { Assistive con- } \\
\text { stant force }\end{array}$ & $\begin{array}{c}\text { Damper against } \\
\text { movement }\end{array}$ & Not clear \\
\hline \multirow[t]{2}{*}{1} & & & & 1 & & & & & & & \\
\hline & & & & 1 & & & & & & & \\
\hline 1 & & & & 1 & & & & 1 & & 1 & \\
\hline \multirow[t]{3}{*}{1} & 1 & & & 1 & & & & 1 & & 1 & \\
\hline & & & & 1 & & & & & & & \\
\hline & & & & 1 & & & & & & & \\
\hline \multirow[t]{8}{*}{1} & 1 & 1 & & & & & & & & & \\
\hline & & & & 1 & & & & & & & \\
\hline & & & & & & & & & & & 1 \\
\hline & & 1 & & & & & & & & & \\
\hline & & & & & & & & & & & 1 \\
\hline & & & & & & & & & & & 1 \\
\hline & & & & & & & & & & & 1 \\
\hline & & & & & & & & & & & 1 \\
\hline 1 & & & & & & & & 1 & & 1 & \\
\hline \multirow[t]{5}{*}{1} & & & & & & & & 1 & & 1 & \\
\hline & & 1 & & & & & & & & & 1 \\
\hline & & & & 1 & & & & & & & \\
\hline & & & & 1 & & & & & & & \\
\hline & & & 1 & & 1 & & & & & & \\
\hline \multirow[t]{2}{*}{1} & 1 & 1 & & & & & & & & & \\
\hline & 1 & & & & & & & & & & \\
\hline 1 & & & & 1 & & & & 1 & & 1 & \\
\hline \multirow[t]{3}{*}{1} & 1 & & & 1 & & & & 1 & & 1 & \\
\hline & & & & 1 & 1 & & & & & & \\
\hline & & & & & & & & & 1 & & \\
\hline 1 & & & & 1 & & & & & & & \\
\hline \multirow[t]{4}{*}{1} & & & & 1 & & & & & & & \\
\hline & 1 & & 1 & & & & 1 & & & & \\
\hline & & & & & & 1 & & & & & \\
\hline & & & & & & 1 & & & & & \\
\hline 1 & & & & & & & & & & & 1 \\
\hline 1 & & & & & & & & & & & 1 \\
\hline \multicolumn{12}{|l|}{1} \\
\hline 1 & & & & & 1 & & & & & & \\
\hline 1 & & & & & & & & & & & 1 \\
\hline
\end{tabular}


Chapter 2

Additional file 2: Reviewed articles (continued)

\begin{tabular}{|c|c|c|c|c|c|c|c|c|c|c|}
\hline \multirow[t]{2}{*}{ Authors } & \multirow[t]{2}{*}{ Year } & \multirow[t]{2}{*}{ Reference } & \multicolumn{8}{|c|}{ TRAINING MODALITY } \\
\hline & & & Passive & Passive-mirrored & Assistive & Active-Assistive & Path Guidance & Corrective & Resistive & Active \\
\hline Krebs et al. & 2008 & 49 & & & & 1 & & & & \\
\hline Krebs et al. & 2008 & 49 & & & & 1 & & & & \\
\hline Krebs et al. & 2008 & 49 & & & & 1 & & & & \\
\hline Song et al. & 2008 & 92 & & & & 1 & & & 1 & \\
\hline Takahashi et al. & 2008 & 96 & & & & 1 & & & & 1 \\
\hline Takahashi et al. & 2008 & 96 & & & & 1 & & & & \\
\hline Colombo et al. & 2008 & 99 & 1 & & & 1 & & & & 1 \\
\hline Colombo et al. & 2008 & 99 & 1 & & & 1 & & & & 1 \\
\hline Treger et al. & 2008 & 106 & 1 & & 1 & & & & & \\
\hline Ellis et al. & 2009 & 80 & & & 1 & & & & 1 & \\
\hline Ellis et al. & 2009 & 80 & & & 1 & & & & & \\
\hline Cordo et al. & 2009 & 82 & 1 & & & & & & & \\
\hline Casadio et al. & 2009 & 98 & & & & 1 & & 1 & 1 & \\
\hline Hu et al. & 2009 & 88 & & & & 1 & & & & \\
\hline Housman et al. & 2009 & 108 & & & 1 & & & & & 1 \\
\hline Posteraro et al. & 2010 & 57 & & & & 1 & & & & 1 \\
\hline Posteraro et al. & 2010 & 57 & & & & 1 & & & & 1 \\
\hline Lo et al. & 2010 & 27 & & & 1 & 1 & & & & \\
\hline Kutner et al. & 2010 & 91 & 1 & & & 1 & & & & 1 \\
\hline Colombo et al. & 2010 & 100 & & & & 1 & & & & \\
\hline Conroy et al. & 2011 & 58 & & & & 1 & & & & 1 \\
\hline Conroy et al. & 2011 & 58 & & & & 1 & & & & 1 \\
\hline Zollo et al. & 2011 & 60 & & & & 1 & & & & \\
\hline Hesse et al. & 2011 & 62 & 1 & 1 & & & & & & \\
\hline Hsieh et al. & 2011 & 64 & 1 & 1 & & & & & & 1 \\
\hline Hsieh et al. & 2011 & 64 & & 1 & & & & & & \\
\hline Burgar et al. & 2011 & 68 & 1 & 1 & & 1 & & & 1 & \\
\hline Burgar et al. & 2011 & 68 & 1 & 1 & & 1 & & & 1 & \\
\hline Abdullah et al. & 2011 & 75 & 1 & & & 1 & & & & 1 \\
\hline Stein et al. & 2011 & 78 & 1 & & & 1 & & & & \\
\hline Merians et al. & 2011 & 84 & & & & 1 & & & & \\
\hline Wei et al. & 2011 & 85 & & & & 1 & & & & \\
\hline Wei et al. & 2011 & 85 & 1 & & & & & & & \\
\hline Lambercy et al. & 2011 & 95 & 1 & & & 1 & & & 1 & \\
\hline Riley et al. & 2011 & 97 & & & & 1 & & & & 1 \\
\hline Masiero et al. & 2011 & 104 & 1 & & 1 & & & & & \\
\hline Bovolenta et al. & 2011 & 107 & & & 1 & 1 & 1 & & & 1 \\
\hline Dipietro et al. & 2012 & 48 & & & 1 & 1 & & & & \\
\hline Dipietro et al. & 2012 & 48 & & & 1 & 1 & & & & \\
\hline Liao et al. & 2012 & 66 & 1 & 1 & & & & & 1 & 1 \\
\hline Hwang et al. & 2012 & 79 & 1 & & & 1 & & & & \\
\hline Hwang et al. & 2012 & 79 & & & & 1 & & & & \\
\hline Duret et al. & 2013 & 44 & & & & 1 & & & & \\
\hline Song et al. & 2013 & 93 & & & 1 & & & & 1 & \\
\hline Byl et al. & 2013 & 112 & & & 1 & & & & & \\
\hline Byl et al. & 2013 & 112 & & 1 & 1 & & & & & \\
\hline Reinkensmeyer et al. & 2012 & 110 & & & 1 & 1 & & & & 1 \\
\hline Wu et al. & 2012 & 67 & 1 & 1 & & & & & 1 & \\
\hline Mazzoleni et al. & 2013 & 59 & & & & 1 & & & & 1 \\
\hline Mazzoleni et al. & 2013 & 59 & & & & 1 & & & & 1 \\
\hline Abdollahi et al. & 2013 & 111 & & & & & & & & \\
\hline Pinter et al. & 2013 & 76 & 1 & & & & & & & \\
\hline Sale et al. & 2012 & 77 & 1 & & 1 & & & & & 1 \\
\hline Rabin et al. & 2012 & 113 & & & 1 & & & & & \\
\hline Pellegrino et al. & 2012 & 61 & & & 1 & & & & & \\
\hline Song et al. & 2013 & 87 & & & 1 & & & & 1 & \\
\hline
\end{tabular}




\begin{tabular}{|c|c|c|c|c|c|c|c|c|c|c|c|}
\hline \multicolumn{12}{|c|}{ FEATURE OF HRI } \\
\hline Passive & $\begin{array}{l}\text { Passive- } \\
\text { mirrored }\end{array}$ & $\begin{array}{c}\text { Spring against } \\
\text { movement }\end{array}$ & $\begin{array}{c}\text { Moving } \\
\text { attractor }\end{array}$ & $\begin{array}{c}\text { Triggered } \\
\text { assistance }\end{array}$ & $\begin{array}{l}\text { Pushing force (in } \\
\text { case of delay) }\end{array}$ & $\begin{array}{l}\text { Emg-pro- } \\
\text { portional }\end{array}$ & Tunnels & $\begin{array}{l}\text { Spring-damper } \\
\text { guidance }\end{array}$ & $\begin{array}{l}\text { Assistive con- } \\
\text { stant force }\end{array}$ & $\begin{array}{c}\text { Damper against } \\
\text { movement }\end{array}$ & Not clear \\
\hline & & & & & 1 & & & 1 & & & \\
\hline & & & & & 1 & & & 1 & & & \\
\hline & & & & & 1 & & & 1 & & & \\
\hline & & 1 & & & & 1 & & & & & \\
\hline & & & & 1 & & & & & & & \\
\hline & & & & 1 & & & & & & & \\
\hline 1 & & & & 1 & & & & & & & \\
\hline 1 & & & & 1 & & & & & & & \\
\hline \multirow[t]{3}{*}{1} & & & & & & & & & & & 1 \\
\hline & & & & & & & & & 1 & & \\
\hline & & & & & & & & & 1 & & \\
\hline \multicolumn{12}{|l|}{1} \\
\hline & & & & & & & 1 & & 1 & 1 & \\
\hline \multicolumn{12}{|c|}{1} \\
\hline & & & & & & & & & 1 & & \\
\hline & & & & & 1 & & & 1 & & & \\
\hline & & & & & 1 & & & 1 & & & \\
\hline & & & & & 1 & & & 1 & & & \\
\hline \multirow[t]{5}{*}{1} & & & & 1 & & & & & & & \\
\hline & & & & 1 & & & & & & & \\
\hline & & & & & 1 & & & 1 & & & \\
\hline & & & & & 1 & & & 1 & & & \\
\hline & & & & & 1 & & & 1 & & & 1 \\
\hline 1 & 1 & & & 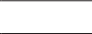 & & & & & & & . \\
\hline \multirow[t]{2}{*}{1} & 1 & & & & & & & & & & 1 \\
\hline & 1 & & & & & & & & & & 1 \\
\hline 1 & 1 & 1 & & & & & & & & & 1 \\
\hline 1 & 1 & 1 & & & & & & & & & 1 \\
\hline \multicolumn{12}{|l|}{1} \\
\hline \multirow[t]{3}{*}{1} & & & & & & & & & & & 1 \\
\hline & & & & & & & & & 1 & & \\
\hline & & & & & & 1 & & & & & \\
\hline \multicolumn{12}{|l|}{1} \\
\hline \multirow[t]{2}{*}{1} & & & 1 & & & & & & & & \\
\hline & & & & 1 & & & & & & & \\
\hline \multirow[t]{4}{*}{1} & & & & & 1 & & & 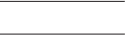 & & & \\
\hline & & & & & & & & & & & 1 \\
\hline & & & & & 1 & & & 1 & & & \\
\hline & & & & & 1 & & & 1 & & & \\
\hline 1 & 1 & & & & & & & & & & 1 \\
\hline \multirow[t]{7}{*}{1} & & & & & & & & & & & 1 \\
\hline & & & & & & & & & & & 1 \\
\hline & & & & 1 & & & & & & & \\
\hline & & 1 & & & & 1 & & & & & \\
\hline & & & 1 & & & & & 1 & 1 & & \\
\hline & 1 & & & & & & & & 1 & & \\
\hline & & & & & & & & & 1 & & 1 \\
\hline \multirow[t]{3}{*}{1} & 1 & 1 & & & & & & & & & \\
\hline & & & & & & & & & & & 1 \\
\hline & & & & & & & & & & & 1 \\
\hline \multicolumn{12}{|l|}{1} \\
\hline \multirow[t]{3}{*}{1} & & & & & & & & & & & 1 \\
\hline & & & & & & & & & 1 & & 1 \\
\hline & & & & & & & & & & & 1 \\
\hline
\end{tabular}




\section{Chapter 2}

Additional file 2: Reviewed articles (continued)

\begin{tabular}{|c|c|c|c|c|c|c|c|c|c|c|}
\hline \multirow[t]{2}{*}{ Authors } & \multirow[t]{2}{*}{ Year } & \multirow[t]{2}{*}{ Reference } & \multicolumn{8}{|c|}{ TRAINING MODALITY } \\
\hline & & & Passive & Passive-mirrored & Assistive & Active-Assistive & Path Guidance & Corrective & Resistive & Active \\
\hline Dohle et al. & 2013 & 114 & & & 1 & 1 & & & & \\
\hline Frisoli et al. & 2012 & 115 & & & 1 & & 1 & & & \\
\hline Colombo et al. & 2013 & 102 & & & & 1 & & & & 1 \\
\hline Colombo et al. & 2013 & 102 & & & & 1 & & & & 1 \\
\hline Hu et al. & 2013 & 116 & & & 1 & & & & & 1 \\
\hline Panarese et al. & 2012 & 101 & & & & 1 & & & & \\
\hline Squeri et al. & 2013 & 74 & & & 1 & & & 1 & & 1 \\
\hline
\end{tabular}


Training modalities: review

\begin{tabular}{|c|c|c|c|c|c|c|c|c|c|c|c|}
\hline \multicolumn{12}{|c|}{ FEATURE OF HRI } \\
\hline Passive & $\begin{array}{l}\text { Passive- } \\
\text { mirrored }\end{array}$ & $\begin{array}{l}\text { Spring against } \\
\text { movement }\end{array}$ & $\begin{array}{l}\text { Moving } \\
\text { attractor }\end{array}$ & $\begin{array}{l}\text { Triggered } \\
\text { assistance }\end{array}$ & $\begin{array}{l}\text { Pushing force (in } \\
\text { case of delay) }\end{array}$ & $\begin{array}{l}\text { Emg-pro- } \\
\text { portional }\end{array}$ & Tunnels & $\begin{array}{l}\text { Spring-damper } \\
\text { guidance }\end{array}$ & $\begin{array}{l}\text { Assistive con- } \\
\text { stant force }\end{array}$ & $\begin{array}{c}\text { Damper against } \\
\text { movement }\end{array}$ & Not clear \\
\hline & & & & 1 & & & & & 1 & & \\
\hline \multicolumn{12}{|c|}{1} \\
\hline \multicolumn{12}{|c|}{1} \\
\hline \multicolumn{12}{|c|}{1} \\
\hline \multicolumn{12}{|c|}{1} \\
\hline \multicolumn{12}{|c|}{1} \\
\hline & & & 1 & & & & & & & 1 & \\
\hline
\end{tabular}





\section{Chapter 3}

Direct effect of a dynamic wrist and hand orthosis on reach and grasp kinematics in chronic stroke

Nijenhuis SM, Prange GB, Stienen AHA, Buurke JH, Rietman JS Proceedings of 14th IEEE International Conference on Rehabilitation Robotics 2015; 


\begin{abstract}
Many stroke patients have impaired arm and hand function. Distal arm and hand devices may support functional use of the upper extremity in activities in daily life. The present study assessed the direct effects of a passive dynamic wrist and hand orthosis on hand and arm movements during the performance of a reach and grasp task in ten mildly to moderately impaired chronic stroke patients. The use of the orthosis resulted in an increase in hand opening, increase in trunk displacement and decrease in elbow ROM during the performance of the reach and grasp task. Therefore, the use of an additional device supporting the arm against gravity should be considered when using such an orthosis in future research, to counteract potential compensatory trunk movements. The findings obtained in this study are being taken into consideration for a longitudinal feasibility study using this orthosis in combination with a computerized gaming environment in chronic stroke at home.
\end{abstract}




\section{INTRODUCTION}

Many stroke survivors have impaired arm and hand function at the affected body side, limiting independent performance of activities in daily life (ADL). Only 5-20\% of the patients have full functional recovery six months after stroke. ${ }^{1}$ In the long run, impaired arm and hand function may result in non-use of the arm and hand in daily life, limiting the independence in ADL even more. ${ }^{2}$ Many physical therapy interventions are available to stimulate restoration of hemiparetic arm and hand function after stroke. ${ }^{3}$ It is known that interventions involving highly repetitive, task-specific training in a motivating environment with active contribution of the patient are important for optimal motor relearning..$^{3-5}$ To enable this kind of intensive treatment, the application of rehabilitation robotics is promising. Several systematic literature reviews have shown the effectiveness of rehabilitation robotics for the hemiparetic arm. ${ }^{6-10}$ The first developed robotic devices mostly focused on training of the proximal arm only. ${ }^{6,11,12}$ However, in recent years a growing number of studies describe interventions involving the distal arm and hand. ${ }^{13-16}$ Including the distal arm and hand is thought to be essential for optimizing the (functional) training leading to a generalization effect of distal arm and hand training to improved control of the entire upper extremity. ${ }^{14}$ An example of a training device for the hand is the SaeboFlex (Saebo Inc., Charlotte NC, USA), which is a custom fabricated passive orthosis assisting in hand opening during grasp and release activities for individuals with moderate to severe hemiparesis. ${ }^{17}$ This orthosis is designed to hold the wrist in extended position allowing functional grasps while supporting finger extension via springs, and showed improvements in hemiparetic upper extremity function after stroke. ${ }^{18,19}$

Most studies involving training devices for the upper extremity so far are performed in the clinic, while only limited studies are available which are performed at home. ${ }^{20}$, ${ }^{21}$ The application of rehabilitation systems in domestic settings with remote monitoring by healthcare professionals reduces the need for one-to-one treatment time, enhances the availability of therapy and enables increased training dose. The present study was performed as part of the first phase of the European SCRIPT project, in which a passive dynamic wrist and hand orthosis was developed for interactive rehabilitation of the arm and hand in chronic stroke at home. ${ }^{22}$ The training emphasizes both hand and wrist movements during functional movements involving reaching and grasping. Since the present study was performed as part of the design phase within the SCRIPT project, we were primarily interested in the direct effects of the orthosis in a small sample of mild to moderate impaired chronic stroke patients, before its clinical potential was examined in a longitudinal feasibility study. The aim of the present study was to assess the direct effects of the orthosis on primarily hand and wrist movements during the performance of a reach and grasp task. In addition, the effects on proximal arm movements and temporal movement patterns were examined. We hypothesized that the use of the orthosis facilitates hand opening and 
wrist extension, which is especially useful in the reach-to-grasp phase in handling objects in ADL. In addition, we aimed to identify prognostic values from participants that benefited most from the support provided by the orthosis. This knowledge might form a basis for understanding or even predicting potential clinical changes when applying the wrist and hand orthosis as a training device.

\section{Methods}

\section{Participants}

Participants were recruited from the Roessingh Rehabilitation Center, Enschede, the Netherlands. Participants were eligible for inclusion if they suffered a first ischemic or hemorrhagic stroke resulting in single-sided hemiparesis between 6 months and 5 years ago, had limited arm and hand function but with at least $15^{\circ}$ active elbow flexion and a quarter range of active finger flexion, and had no shoulder pain or co-morbidities. The study was approved by the local medical ethical committee and all participants provided written informed consent before participation.

\section{Study design}

Participants were measured in the research lab during a single session cross-sectional evaluation. In order to assess the functional capacity of the participants, two clinical tests were carried out. The upper extremity motor section of the Fugl-Meyer assessment (FM, max. 66 points) was used to measure arm and hand function, ${ }^{23,24}$ and the Action Research Arm Test (ARAT, max. 57 points) was used to assess dexterity. ${ }^{25,26}$ Upper extremity movement kinematics were measured during a reach and grasp task, mimicking a functional movement. This task was performed in two conditions in randomized order: without and with wearing a dynamic wrist and hand orthosis.

\section{Dynamic wrist and hand orthosis}

The dynamic wrist and hand orthosis (Figure 3.1) is a non-actuated custom-designed exoskeleton which fits onto the forearm and hand and compensates for impairments caused by spasticity and abnormal synergies. ${ }^{27}$ These impairments are characterized by involuntary flexion torques, which disturb normal hand function. The orthosis offsets these undesired torques. It interacts physically with the participant by applying external extension torques to the wrist and extension forces to the fingers via leaf springs and elastic tension cords. The amount of support can be adapted to provide more or less of an offset force, to enable participants to train to their maximal capacity. During the reach and grasp task, the amount of support was set based on a simple test to reach and grasp a soft ball several times, and was increased (decreased) when this test was perceived to be too difficult (easy), in addition to the participant's opinion about comfort. 


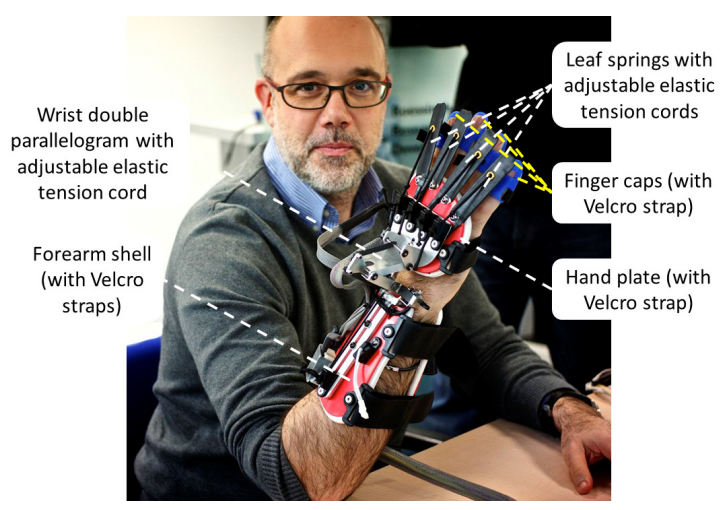

Figure 3.1 Dynamic wrist and hand orthosis

\section{Procedures}

After the assessment of the FM and ARAT, the reach and grasp test was performed. The participant sat on a chair, in front of a table. The starting position of the participant was with the upper arm aligned to the trunk, with the elbow in $90^{\circ}$ flexion, and with the palm of the hand resting on the table in front of the participant (Position A in Figure 3.2). A bottle with diameter of $6 \mathrm{~cm}$ was located at position $B$ on the table, right in front of the participant. The distance of the starting position $A$ to position $B$ was determined by near-maximal $(80 \%)$ active forward reach of each participant at the start of the task. The participant was instructed to perform the following standardized task with the affected arm and hand, at a comfortable, self-selected speed, for about ten subsequent repetitions:

1) Reach to position $B$, grasp the bottle

2) Hold the bottle and move the hand to position $A$

3) Bring the hand with bottle back to position $B$, release the bottle

4) Bring the hand back to position $\mathrm{A}$ (starting position)

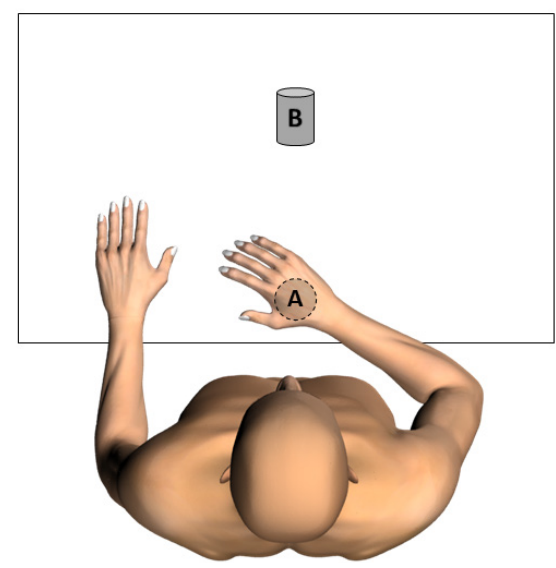

Figure 3.2 Measurement setup during reach and grasp task 


\section{Measurements}

During the performance of the reach and grasp task, upper extremity movement kinematics were recorded using a 3D motion analysis system (VICON MX13+ motion capture system, Oxford Metrics, Oxford, UK). This system uses six infrared cameras to capture movements of the arm and hand, by recording reflective markers placed on the upper extremity according to guidelines of the International Society of Biomechanics for the $\mathrm{arm}^{28}$ and an adapted version of a validated marker model for the hand. ${ }^{29}$

\section{Data analysis}

Recorded VICON data were analyzed using VICON Nexus 1.8.2 and custom-written software in MATLAB (MathWorks Inc., Natick, Massachusetts, USA). The data were filtered with a second order low pass Butterworth filter of $20 \mathrm{~Hz}$ and zero phase shift. For each participant, all ten task repetitions were analyzed for both conditions, and were sorted from largest to smallest hand opening. For each participant, the average of the first seven repetitions (with largest hand opening) was calculated and used for further analysis. For participants with less than seven useful repetitions available (for example because of a poor data sample), the average of the available data were used, with a minimum of three complete repetitions.

The primary parameters which were calculated from the 3D position data measured during the reach and grasp task focused on the distal arm and hand. The range of motion (ROM) of the wrist was calculated by subtracting the smallest angle from the largest angle between the forearm and hand during the total movement task. The maximum hand opening ( $\mathrm{HO})$ was determined as the maximum Euclidean distance between the tips of the thumb and middle finger during the first phase of the movement task, the reach-to-grasp phase. The moment of maximal wrist extension (WE) and moment of maximal HO were also calculated in the reach-to-grasp phase, in which it is expected that participants might experience most benefit from wearing the orthosis. This reach-to-grasp phase was defined as the movement from start position A $(0 \%)$ towards the target position $B(100 \%)$. The start and end of the reach-to-grasp phase were defined as the moment when the tangential velocity of the hand marker exceeded (start) and came below (end) $2 \%$ of the maximum velocity in the first reaching phase. ${ }^{30}$ In addition to distal arm and hand movement parameters, the following parameters were also calculated over the complete reach and grasp task. ROM of the elbow was determined between the largest and smallest joint angle between the lower arm and the upper arm during the total movement task. Maximal forward trunk displacement (TD) was defined as the maximal displacement of the trunk marker in the sagittal plane from the start position during the total movement task. The movement time (MT) was defined as the time participants needed to perform the total reach and grasp task. Movement onset was defined as the moment when the tangential velocity of the hand marker exceeded $2 \%$ of the maximum velocity in the first reaching phase. Movement offset was determined when 
the velocity was below $2 \%$ of the maximum velocity in the last returning phase. ${ }^{30}$ Movement smoothness (MS) was quantified by jerk, and calculated as number of movement units ( $\mathrm{nmu}$ ) in the tangential velocity profile of the hand marker. To define a movement unit, this profile was searched for local minima and maxima: a difference between a minimum and next maximum value that exceeded the amplitude limit of $20 \mathrm{~mm} / \mathrm{s}$ indicated a velocity peak. The time between two subsequent peaks was at least 150 milliseconds. ${ }^{30}$

\section{Statistical analysis}

Individual scores per condition were averaged across participants and reported as median with interquartile range (IQR, $25^{\text {th }}-75^{\text {th }}$ percentile). Outcomes were non-parametrically tested for statistical significance due to the small sample size. The Wilcoxon signed ranks test was used to determine the differences between both conditions (with and without orthosis) on kinematic parameters. In addition, the relationship between clinical outcome scores (FM and ARAT) and kinematic differences between condition (with versus without orthosis) was assessed with Spearman's correlation coefficient. Statistical analysis was performed with IBM SPSS Statistics 19 for Windows and effects were considered statistically significant for $\mathrm{P}<0.05$.

\section{RESULTS}

\section{Participants}

Ten chronic stroke patients were included in the study. Participant characteristics and the clinical FM and ARAT scores are presented in Table 3.1. Three participants had moderate hemiparesis (FM $26 \leq F M \leq 50$ ) and seven had mild hemiparesis (FM > 50). Due to partly missing data of the wrist marker in four participants in the condition with orthosis, data of the moment of maximal WE are presented from seven participants and ROM of the wrist and elbow from six participants.

Table 3.1 Participant characteristics

\begin{tabular}{lc}
\hline & $\boldsymbol{N}=\mathbf{1 0}$ \\
\hline Gender & 7 male / 3 female \\
Age (years) & 60 (IQR: $49-70)$ \\
Months post stroke & 15 (IQR: $14-25)$ \\
Affected body side & 2 right / 8 left \\
Dominant arm & 9 right / 1 left \\
FM (max. 66) & 52.5 (IQR: $46-57)$ \\
ARAT (max. 57) & 41.5 (IQR: $31.5-50.5)$ \\
\hline
\end{tabular}

Three participants showed an increase in wrist ROM (17.81, 18.19 and 5.01 degrees) using the orthosis, although three other participants showed a decrease in wrist ROM (-13.80, 
-5.02 and -7.27 degrees) while wearing the orthosis. All ten participants increased their maximal hand opening using the orthosis, ranging from 3.24 to $38.25 \mathrm{~mm}$ increase compared to without orthosis.

On group level, a significant difference was found for maximal hand opening, ROM of the elbow and trunk displacement when comparing both conditions: without and with wearing orthosis (Table 3.2 and Figure 3.3). Maximal hand opening improved with a group median of $11.98 \mathrm{~mm}$ in the condition with orthosis $(P=0.005)$.

Table 3.2 Median with IQR of kinematic outcomes without and with orthosis

\begin{tabular}{lccc}
\hline & N & Without orthosis & With orthosis \\
\hline Max. HO (mm) & 10 & $172.21(156.23-181.47)$ & $184.59(169.45-193.71)$ \\
Moment max. HO (\%) & 10 & $58.68(50.21-72.53)$ & $61.07(50.78-65.46)$ \\
Moment max. WE (\%) & 7 & $86.40(67.08-99.30)$ & $81.92(72.55-98.87)$ \\
ROM Wrist (degrees) & 6 & $54.25(35.62-60.10)$ & $50.72(38.13-70.50)$ \\
ROM Elbow (degrees) & 6 & $50.54(32.18-55.43)$ & $46.33(28.61-49.92)$ \\
TD (mm) & 10 & $64.13(19.61-123.03)$ & $85.40(26.26-178.03)$ \\
MT (s) & 10 & $6.50(5.30-9.27)$ & $7.54(6.16-9.13)$ \\
MS (nmu) & 10 & $15.50(11.96-23.14)$ & $18.07(14.39-21.14)$ \\
\hline
\end{tabular}

a Significant difference

Median differences with versus without orthosis

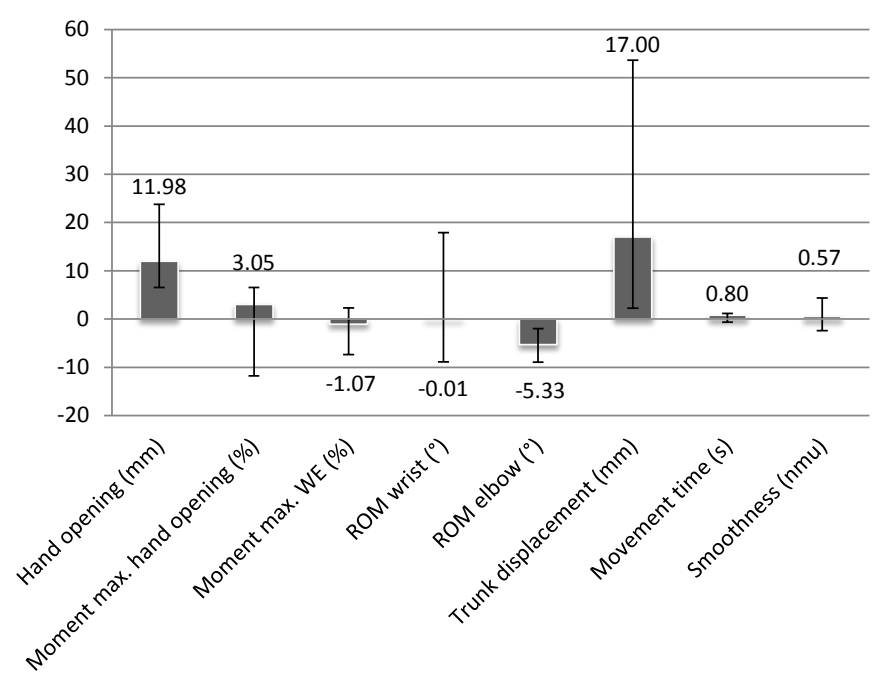

Figure 3.3 Median differences with IQR ranges with versus without orthosis Positive values represent an increase in outcome with orthosis, negative values represent a decrease in outcome with orthosis 
Wearing the orthosis had a negative effect on the ROM of the elbow $(P=0.028)$ and trunk displacement $(P=0.017)$, indicating that participants compensated more while wearing the orthosis (median increase of $17.00 \mathrm{~mm}$ on TD and median decrease of 5.33 degrees on elbow ROM). The other outcomes did not show statistically significant differences across both conditions $(P \geq 0.285)$. Correlations between clinical scores and kinematic differences with versus without orthosis were also calculated, but no significant correlations were found $(P \geq 0.130)$.

\section{Discussion}

The aim of the present study was to assess the direct effects of a dynamic wrist and hand orthosis on hand and arm movements during the performance of a reach and grasp task in mildly to moderately affected stroke patients. As expected, the use of the orthosis facilitated hand opening with a median improvement of $11.98 \mathrm{~mm}$. This increase might be useful in the reach-to-grasp phase in handling objects in daily life. It is expected that the orthosis will also facilitate functional use of the impaired hand during training, by assisting finger extension. This was also reported by other studies using other passive devices assisting in finger extension. ${ }^{19,31}$ One also showed an increase in ROM in opening and closing of the hand after a training period using the device. ${ }^{31}$

The moment of maximal hand opening and maximal wrist extension in the reach-tograsp phase did not change when wearing the orthosis. Previous research has shown that maximum hand aperture occurs at approximately 60 to $80 \%$ of the reach-to-grasp phase and depends on the object size. ${ }^{32,33}$ In our study, maximum hand opening took place at median $58.68 \%$ without orthosis and $61.07 \%$ with orthosis, and maximum wrist extension at $86.40 \%$ and $81.92 \%$, respectively. This indicates that timing of hand opening and wrist extension in this sample of stroke patients occurred in a pattern similar of that of healthy persons. Wearing the orthosis did not affect the temporal pattern.

While wearing the wrist and hand orthosis, participants showed more compensation strategies during the performance of the reach and grasp task, indicated by an increase in trunk displacement and decrease in elbow ROM. This compensation strategy might be related to the weight of the orthosis on the arm (about $0.7 \mathrm{~kg}$ ), which could have impeded the performance of the task on proximal level. Future design of this orthosis should therefore focus on reducing the weight of the orthosis, to minimize compensatory trunk movements. The use of devices supporting the weight of the proximal arm have been shown to facilitate active arm movement excursions without impairing motor control, during reach and retrieval movements in a single-session evaluation. ${ }^{34}$ The positive influence of gravity support is also shown in longitudinal studies, showing improvements on the FM score after a period of gravity-compensated training for the affected arm. ${ }^{11,35}$ The application of both proximal and 
distal arm and hand support is needed to facilitate both arm and hand movements, allowing more functional practice. This should also be taken into account in current physical therapy programs involving other distal arm and hand training devices (for example ${ }^{19}$ ), in which specific attention is needed to correct abnormal movements of the trunk and proximal arm.

Unfortunately, wrist ROM data was available of only six participants, showing that the use of the orthosis did not result in an increase of wrist ROM on group level. However, on individual level, a large amount of variation between participants was found. Three participants showed an increase and three participants a decrease in wrist ROM. The maximum wrist ROM required to perform activities of daily living is between 60 degrees of extension and 54 degrees of flexion. ${ }^{36}$ Taking this into account, an improvement of 17.81 and 18.19 degrees in wrist ROM for two participants included in this study is a major improvement for them. In addition, it might be true that some of the participants included in this study were already able to move the wrist within functional ranges by themselves for this specific reach and grasp task, and did not need the benefit from the orthosis considering the wrist extension support.

Correlation analysis did not reveal any strong associations between clinical FM or ARAT scores and differences in kinematic outcomes with versus without orthosis. This could probably be related to a select group of mild to moderate stroke patients included in this study, with FM scores ranging from 37 to 57 points, with the majority of the patients having mild stroke. No severe stroke patients (FM scores < 26) were included in this study. This is because the use of the orthosis requires that the patient has at least some flexion activity in order to make grasping movements possible. It is though possible that more severely affected patients could also benefit from the support of the orthosis. Furthermore, our findings indicate that patients that might benefit from this type of support cannot be specified in terms of general clinical scales. Instead, a more detailed assessment on impairment level is needed. For example the assessment of (high) wrist flexor tone and/or (low) wrist extensor activity might be useful to identify those who could benefit most from the support of the orthosis and therefore could have the largest potential for clinical improvement when applying the orthosis as a training device.

\section{CONCLUSION}

The use of a dynamic wrist and hand orthosis showed a positive direct effect on hand opening during the performance of a reach and grasp task in patients with chronic stroke. The orthosis had a negative effect on trunk displacement and elbow ROM, which is probably related to the weight of the orthosis on the arm. The use of an additional device supporting the arm against gravity should therefore be considered when using such an orthosis in future research, to counteract potential compensatory trunk movements. Furthermore, 
the temporal movement patterns, which resembled normal movement patterns, remained unaffected by wearing the orthosis. Unfortunately, no conclusions can be drawn at this point about which factors, in terms of general clinical scales, describe the participants that might benefit most from the support of this orthosis. The findings obtained in this study will be taken into consideration when preparing a longitudinal feasibility study focusing at user acceptance and potential clinical benefits after a training period with the SCRIPT orthosis in combination with a computerized gaming environment in chronic stroke at home. This will include the use of an additional weight support device and inclusion of a wide range of participants.

\section{REFERENCES}

1. Kwakkel G, Kollen BJ, van der Grond J and Prevo AJ. Probability of regaining dexterity in the flaccid upper limb: impact of severity of paresis and time since onset in acute stroke. Stroke. 2003; 34: 2181-6.

2. Taub E, Uswatte G, Mark V and Morris D. The learned nonuse phenomenon: implications for rehabilitation. Eura Medicophys. 2006; 42: 241-55.

3. Veerbeek JM, van Wegen $E$, van Peppen $R$, et al. What is the evidence for physical therapy poststroke? A systematic review and meta-analysis. PLoS One. 2014; 9: e87987.

4. Schaechter JD. Motor rehabilitation and brain plasticity after hemiparetic stroke. Progress in Neurobiology. 2004; 73: 61-72.

5. Kwakkel G. Impact of intensity of practice after stroke: issues for consideration. Disabil Rehabil. 2006; 28: 823-30.

6. Prange GB, Jannink MJ, Groothuis-Oudshoorn CG, Hermens HJ and ljzerman MJ. Systematic review of the effect of robot-aided therapy on recovery of the hemiparetic arm after stroke. J Rehabil Res Dev. 2006; 43: 171-84.

7. Kwakkel G, Kollen BJ and Krebs HI. Effects of robot-assisted therapy on upper limb recovery after stroke: a systematic review. Neurorehabil Neural Repair. 2008; 22: 11121.

8. Mehrholz J, Pohl M, Platz T, Kugler J and Elsner B. Electromechanical and robot-assisted arm training for improving activities of daily living, arm function, and arm muscle strength after stroke. Cochrane Database Syst Rev. 2015; 11: CD006876.

9. Basteris A, Nijenhuis SM, Stienen AH, Buurke JH, Prange GB and Amirabdollahian F. Training modalities in robot-mediated upper limb rehabilitation in stroke: a framework for classification based on a systematic review. J Neuroeng Rehabil. 2014; 11: 111.

10. Maciejasz P, Eschweiler J, Gerlach-Hahn K, Jansen-Troy A and Leonhardt S. A survey on robotic devices for upper limb rehabilitation. Journal of Neuroengineering and Rehabilitation. 2014; 11: 3.

11. Amirabdollahian F, Loureiro R, Gradwell E, Collin C, Harwin W and Johnson G. Multivariate analysis of the Fugl-Meyer outcome measures assessing the effectiveness of GENTLE/S robot-mediated stroke therapy. J Neuroeng Rehabil. 2007; 4: 4.

12. Stein J, Krebs HI, Frontera WR, Fasoli SE, Hughes R and Hogan N. Comparison of two techniques of robot-aided upper limb exercise training after stroke. Am J Phys Med Rehabil. 2004; 83: 720-8.

13. Lo AC, Guarino PD, Richards LG, et al. Robot-assisted therapy for long-term upper-limb impairment after stroke. N Engl J Med. 2010; 362: 1772-83.

14. Balasubramanian S, Klein J and Burdet E. Robot-assisted rehabilitation of hand function. 
Curr Opin Neurol. 2010; 23: 661-70.

15. Klamroth-Marganska V, Blanco J, Campen K, et al. Three-dimensional, task-specific robot therapy of the arm after stroke: a multicentre, parallel-group randomised trial. The Lancet Neurology. 2014; 13: 159-66.

16. Lum PS, Godfrey SB, Brokaw EB, Holley RJ and Nichols D. Robotic approaches for rehabilitation of hand function after stroke. American journal of physical medicine \& rehabilitation / Association of Academic Physiatrists. 2012; 91: S242-54.

17. Hoffman HB and Blakey GL. New design of dynamic orthoses for neurological conditions. NeuroRehabilitation. 2011; 28: 55-61.

18. Jeon $\mathrm{HS}$, Woo YK, Yi CH, et al. Effect of intensive training with a spring-assisted hand orthosis on movement smoothness in upper extremity following stroke: a pilot clinical trial. Topics in Stroke Rehabilitation. 2012; 19: 320-8.

19. Farrell JF, Hoffman HB, Snyder JL, Giuliani CA and Bohannon RW. Orthotic aided training of the paretic upper limb in chronic stroke: results of a phase 1 trial. NeuroRehabilitation. 2007; 22: 99-103.

20. Sivan M, Gallagher J, Makower S, et al. Home-based Computer Assisted Arm Rehabilitation (hCAAR) robotic device for upper limb exercise after stroke: results of a feasibility study in home setting. Journal of Neuroengineering and Rehabilitation. 2014; 11: 163.

21. Laver KE, Schoene D, Crotty M, George S, Lannin NA and Sherrington C. Telerehabilitation services for stroke. Cochrane Database Syst Rev. 2013; 12: CD010255.

22. Amirabdollahian F, Ates S, Basteris A, et al. Design, development and deployment of a hand/wrist exoskeleton for home-based rehabilitation after stroke - SCRIPT project. Robotica. 2014; 32: 1331-46.

23. Fugl-Meyer AR, Jaasko L, Leyman I, Olsson S and Steglind S. The post-stroke hemiplegic patient. 1. A method for evaluation of physical performance. Scand J Rehabil Med. 1975; 7: 13-31.

24. Deakin A, Hill H and Pomeroy VM. Rough Guide to the Fugl-Meyer Assessment: Upper limb section. Physiotherapy. 2003; 89: 751-63.

25. Carroll D. A Quantitative Test of Upper Extremity Function. J Chronic Dis. 1965; 18: 47991.

26. Yozbatiran N, Der-Yeghiaian L and Cramer SC. A standardized approach to performing the action research arm test. Neurorehabil Neural Repair. 2008; 22: 78-90.

27. Ates S, Lobo-Prat J, Lammertse P, van der Kooij H and Stienen AH. SCRIPT Passive Orthosis: Design and technical evaluation of the wrist and hand orthosis for rehabilitation training at home. IEEE International Conference on Rehabilitation Robotics. 2013; 2013: 1-6.

28. Wu G, van der Helm FC, Veeger HE, et al. ISB recommendation on definitions of joint coordinate systems of various joints for the reporting of human joint motion--Part II: shoulder, elbow, wrist and hand. J Biomech. 2005; 38: 981-92.

29. Metcalf CD, Notley SV, Chappell PH, Burridge JH and Yule VT. Validation and application of a computational model for wrist and hand movements using surface markers. IEEE Trans Biomed Eng. 2008; 55: 1199-210.

30. Alt Murphy M, Willen $C$ and Sunnerhagen KS. Kinematic variables quantifying upperextremity performance after stroke during reaching and drinking from a glass. Neurorehabilitation and Neural Repair. 2011; 25: 71-80.

31. Brokaw EB, Black I, Holley RJ and Lum PS. Hand Spring Operated Movement Enhancer (HandSOME): a portable, passive hand exoskeleton for stroke rehabilitation. IEEE transactions on neural systems and rehabilitation engineering : a publication of the IEEE Engineering in Medicine and Biology Society. 2011; 19: 391-9.

32. Jeannerod M. The timing of natural prehension movements. Journal of motor behavior. 
1984; 16: 235-54.

33. Meulenbroek RG, Rosenbaum DA, Jansen C, Vaughan J and Vogt S. Multijoint grasping movements. Simulated and observed effects of object location, object size, and initial aperture. Experimental brain research. 2001; 138: 219-34.

34. Prange GB, Jannink MJ, Stienen AH, van der Kooij $H$, ljzerman MJ and Hermens HJ. Influence of gravity compensation on muscle activation patterns during different temporal phases of arm movements of stroke patients. Neurorehabil Neural Repair. 2009; 23: 478-85.

35. Prange GB, Kottink Al, Buurke JH, et al. The Effect of Arm Support Combined With Rehabilitation Games on Upper-Extremity Function in Subacute Stroke: A Randomized Controlled Trial. Neurorehabil Neural Repair. 2015; 29: 174-82.

36. Ryu JY, Cooney WP, 3rd, Askew LJ, An KN and Chao EY. Functional ranges of motion of the wrist joint. The Journal of hand surgery. 1991; 16: 409-19. 



\section{Chapter 4}

Feasibility study into self-administered training at home using an arm and hand device with motivational gaming environment in chronic stroke

Nijenhuis SM, Prange GB, Amirabdollahian F, Sale P, Infarinato F, Nasr N, Mountain G, Hermens HJ, Stienen AHA, Buurke JH, Rietman JS Journal of NeuroEngineering and Rehabilitation 2015; 12:89 


\begin{abstract}
Background: Assistive and robotic training devices are increasingly used for rehabilitation of the hemiparetic arm after stroke, although applications for the wrist and hand are trailing behind. Furthermore, applying a training device in domestic settings may enable an increased training dose of functional arm and hand training. The objective of this study was to assess the feasibility and potential clinical changes associated with a technologysupported arm and hand training system at home for patients with chronic stroke.
\end{abstract}

Methods: A dynamic wrist and hand orthosis was combined with a remotely monitored user interface with motivational gaming environment for self-administered training at home. Twenty-four chronic stroke patients with impaired arm/hand function were recruited to use the training system at home for six weeks. Evaluation of feasibility involved training duration, usability and motivation. Clinical outcomes on arm/hand function, activity and participation were assessed before and after six weeks of training and at two-month followup.

Results: Mean System Usability Scale score was 69\% (SD 17\%), mean Intrinsic Motivation Inventory score was 5.2 (SD 0.9) points, and mean training duration per week was 105 (SD 66) minutes. Median Fugl-Meyer score improved from 37 (IQR 30) pre-training to 41 (IQR 32) post-training and was sustained at two-month follow-up (40 (IQR 32)). The Stroke Impact Scale improved from 56.3 (SD 13.2) pre-training to 60.0 (SD 13.9) post-training, with a trend at follow-up (59.8 (SD 15.2)). No significant improvements were found on the Action Research Arm Test and Motor Activity Log.

Conclusions: Remotely monitored post-stroke training at home applying gaming exercises while physically supporting the wrist and hand showed to be feasible: participants were able and motivated to use the training system independently at home. Usability shows potential, although several usability issues need further attention. Upper extremity function and quality of life improved after training, although dexterity did not. These findings indicate that home-based arm and hand training with physical support from a dynamic orthosis is a feasible tool to enable self-administered practice at home. Such an approach enables practice without dependence on therapist availability, allowing an increase in training dose with respect to treatment in supervised settings.

Trial registration: This study has been registered at the Netherlands Trial Registry (NTR): NTR3669 


\section{BACKGROUND}

In the chronic phase after stroke, most people still have motor problems, ${ }^{1}$ leading to difficulties in performing activities of daily living (ADL). Good arm and hand motor function is essential to perform ADL independently. Therefore, restoration of arm and hand function is a major objective in stroke rehabilitation. Research into motor relearning and cortical reorganization after stroke has provided a neurophysiological basis for those aspects that are important to stimulate restoration of arm function:2, 3 functional exercises, at high intensity and with active involvement of the patient within a motivating environment. ${ }^{4,5}$

New ways of providing healthcare services, such as teleconsultation and remote monitoring and treatment in the patient's home, often referred to as telerehabilitation, is widely considered as having a bright future in the framework of an innovative rehabilitative approach. ${ }^{6}$ The use of a rehabilitation service within the home allows the user to exercise independently, in an intensive, active and functional way, in a familiar environment while having continuous access to training tools. This gives the patient a sense of control and autonomy, which might also contribute to a better treatment outcome. ${ }^{7}$ To enhance high training adherence, exercises should be provided in a motivating training environment, for instance via computerized gaming including feedback about performance. ${ }^{8}$ Furthermore, a computerized telerehabilitation service enables remote monitoring of movements and remote offline (indirect) supervision by a therapist. This should reduce the need for one-toone treatment time and home visits. This can help relieve the pressure on today's healthcare system which is challenged by an ageing society and increasing long-term conditions such as stroke. ${ }^{9}$ It may also enable prolonged rehabilitation for those who may not be able to access it due to resource and service restrictions within the healthcare system. However, telerehabilitation for stroke is still in its infancy. Two reviews confirmed that only limited numbers of telerehabilitation studies in the stroke domain are currently available. ${ }^{10}$, ${ }^{11}$ More research is required including utilization, ${ }^{10}$ effectiveness and satisfaction ${ }^{11}$ of telerehabilitation services after stroke. Thirteen of the 16 studies within both reviews involved telerehabilitation programs with direct, online supervision, such as videoconsulting or telephone calls. However, this still relies on therapist availability. Interestingly, both reviews did not address the effective training duration as performed by participants during the interventions, which is an essential factor in rehabilitation since this is (in relation to intensity of training) a prerequisite for motor relearning. In addition, this is of particular interest for studies involving remote treatment and offline supervision.

Developments in technology-mediated rehabilitation have made it possible to use rehabilitation robotics to provide safe and intensive training to people with mild to severe motor impairments after neurologic injury. ${ }^{12,}{ }^{13}$ Such devices can provide high-intensity, repetitive, task-specific, interactive training of the impaired upper extremity, and have the 
potential to more accurately quantify therapy and monitor patients' progress. Rehabilitation robotics has been shown to be effective for the hemiparetic arm. ${ }^{14-20}$ However, many training studies using robotic devices focus on either the proximal or distal arm only. ${ }^{14,17,21}$ To improve independent use of the upper extremity in daily life, it is important to include functional movements of both the proximal and distal arm and hand into post-stroke training. ${ }^{22,23}$ Two examples are The Activities of Daily Living Exercise Robot (ADLER) ${ }^{24}$ and Gentle/G system ${ }^{25}$ which have the ability to train both reaching and grasping movements. A next step would be to use such technology at home to support self-administered training, without requiring direct therapist involvement continuously. This requires a training device to be compact and easily transportable.

The current study aims to address the previously mentioned aspects: using a dynamic wrist and hand orthosis in a motivational gaming environment for home rehabilitation to enable and support a high dose of self-administered training, facilitating both proximal and distal arm and hand exercises. In addition, participants are challenged to exercise at their maximum capacity. One of the major issues for the success of telerehabilitation concerns the question whether patients not only accept the technology and profit from it, but also whether they can effectively use the system, and in what dose. This is especially relevant in this study, in which participants were exposed to independent training at home, without direct supervision, using a device which physically interacts with the arm and hand. Since the technology-supported telerehabilitation system for the arm and hand was developed specifically for this study, the evaluation fits best within the first two stages of telemedicine evaluation. The first stage focuses on the feasibility and usability of the technology used in an experimental design with a small number of subjects. In the second stage, potential working mechanisms will be explored in a small group of subjects. ${ }^{26}$ Therefore, the objective of this study was to examine feasibility (user acceptance, effective use) and potential clinical changes (in arm and hand function) of a technology-supported arm and hand training system at home in chronic stroke.

\section{Methods}

\section{Participants}

Three clinical sites in Europe (rehabilitation center Het Roessingh, Enschede, the Netherlands; IRCCS San Raffaele Pisana, Rome, Italy; and the University of Sheffield, Sheffield, United Kingdom) were involved in participant recruitment. Participants were recruited from local rehabilitation centers and regional hospitals and through private physical therapy providers. Inclusion criteria were: (1) patients had to be between 6 months and 5 years after stroke; (2) between 18 and 80 years of age; (3) clinically diagnosed with partial central paresis of the arm or hand due to stroke, but with at least $15^{\circ}$ active elbow flexion and active finger flexion of at least a quarter of the passive range of motion; (4) living at home with internet access; (5) 
having a carer who is co-resident or closely involved in their care; (6) able to understand and follow instructions; (7) no additional orthopedic, neurological, or rheumatologic disease of the upper extremity; (8) and absence of severe neglect or (uncorrected) visual impairments. All participants provided written informed consent before participation. The study protocol was approved by the local ethics committees in all three sites.

\section{Study design}

This feasibility study has a longitudinal design. Participants trained at home for six weeks using the newly developed training system. Arm and hand function was evaluated one week before training (T01), within one week after training (T08), and at two-month follow-up after the end of training (T15). User acceptance (motivation and usability) was evaluated within one week after training (T08). The training dose was automatically stored by the system during the six weeks of training.

\section{Intervention}

Participants trained at home using games while they were supervised remotely, indirectly, by a healthcare professional (HCP). This means that the participant and the HCP do not need to be online at the same time. The HCPs involved in this study were trained clinical researchers (human movement scientists), physical therapists or occupational therapists. All participants were told that they could train at the time of day they preferred. The general recommendation for training was about 30 minutes of exercise per day, six days per week. They were allowed to practice additionally if they wished to. Firstly, two professionals installed and initialized the training system at the participant's home. This involved checking that the system was fully operational and providing instructions to the participant and a family carer regarding safe usage. Instructions for usage were shown within the patient user interface of the system, and participants were also provided with a hard copy user manual. This user manual also included a telephone number which participants could call in case of technical failures or other needs for assistance of the system. During the first week following installation, the HCP contacted each participant twice to ensure their competence with the training system.

The training system (Figure 4.1) developed within the SCRIPT (Supervised Care and Rehabilitation Involving Personal Telerobotics) project ${ }^{27}$ comprised: (a) a computer, (b) a touchscreen, (c) the Saebo Mobile Arm Support (SaeboMAS) (Saebo Inc., Charlotte NC, USA) and $(d)$ the SCRIPT dynamic wrist and hand orthosis. ${ }^{28}$ The SaeboMAS was used for gravity compensation of the proximal arm. The wrist and hand orthosis is a custom-designed exoskeleton which fits onto the forearm and hand. The mechanical design of the orthosis, in combination with the use of the SaeboMAS, allowed movements of the arm, wrist and hand within functional ranges during training. Trunk movements were not constrained. The 
orthosis interacted physically with the participant by providing extension forces to the wrist and fingers via passive leaf springs and elastic tension cords ${ }^{28}$. The amount of support could be adjusted to provide more or less of an offset force, to enable participants to train to their maximal capacity, with as little support as possible. For safety reasons, this amount of support was set by the HCP at the start of the training, based on a simple test to reach and grasp a soft ball several times. The amount of support was increased (decreased) when this test was perceived to be too difficult (easy), in addition to the participant's opinion about comfort. The orthosis was equipped with sensors ${ }^{28}$ to measure the range of motion of the wrist and fingers, and connected to the computer to allow game control and feedback on performance. The computer contained all the software components needed to complete a training session, including a user interface and games. The patient user interface, which was managed via a touchscreen, allowed the participant to select a game, play the game, and review his or her performance history (game scores and training duration). It also enabled the participant to contact the HCP by sending a message.

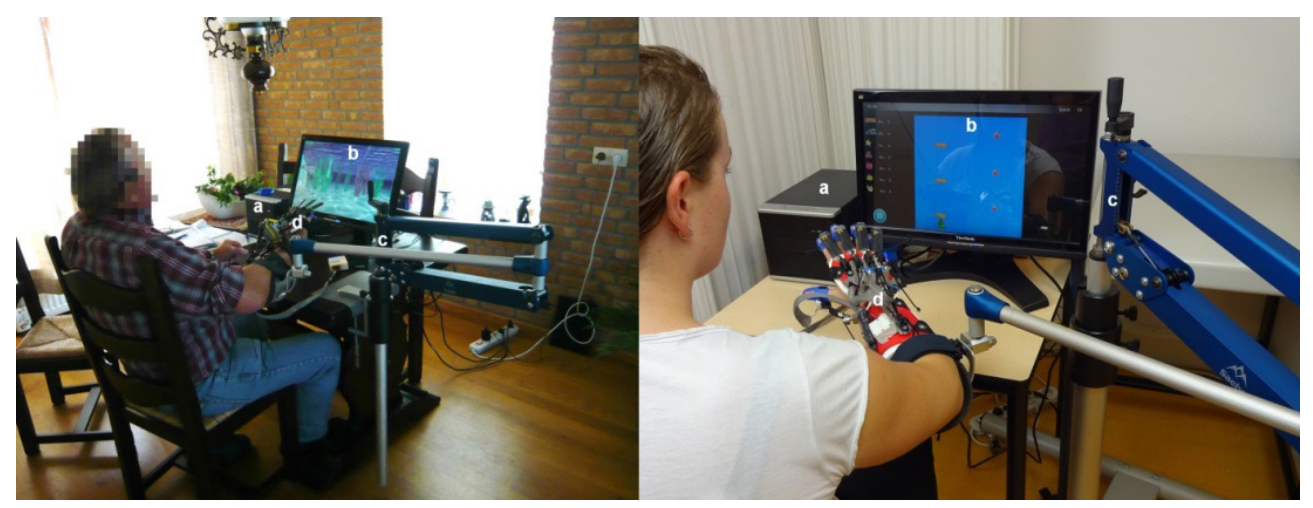

Figure 4.1 Technology-supported training system

Left: training system in use at participant's home, right: more detailed overview of specific components: (a) Computer containing user interface and games, (b) Touchscreen showing one of the games, (c) SaeboMAS, (d) SCRIPT wrist and hand orthosis

Three games with various categories were available for practice. The key gestures in the games were hand opening and closing (grasping), wrist flexion and extension, forearm pronation and supination, and elbow and shoulder movements (reaching). The integrated sensors in the orthosis enabled game control by active execution of gestures (for example wrist flexion or extension corresponded with an avatar diving under or jumping over obstacles on the screen) beyond a threshold of $80 \%$ of the participant's active range of motion. In this way, participants were challenged to exercise at their maximum capacity. This threshold was assessed prior to game playing through a calibration procedure of a few minutes, involving the gestures relevant for the game to be played. The main goals of the games were to provide a fun and engaging experience for interaction while assigning 
a task to the participant, and providing feedback about performance. In addition, visual (e.g. representation of scores during and after exercising) and auditory (e.g. applause and sound effects for correct movements) feedback was provided within the games to keep the participants motivated and engaged in training. The games consisted of various categories, which were classified in a schedule ranked according to increasing complexity. Games were considered more complex when they required multiple planes of movement (from 1D to $3 D$ ), when the number of gestures involved to control the game were increased, or when more difficult movements were needed (progression from proximal to distal movements, and gross to fine manipulation). This game difficulty schedule was used by the HCP weekly to provide the correct game categories to each participant. The HCP adjusted the training program remotely, by accessing the HCP user interface. The HCP visited this user interface daily, to check on potential messages from participants, and to follow the participants' progress and training duration remotely. At that time, participants did not need to be online. If the HCP noticed no or low use, he or she sent a motivational message to the participant. The participant received this message the next time when he or she started a new training session. The decision to provide more complex categories of games was supported and verified by weekly home visits of the HCP to the participant's home. During these weekly home visits participants performed a training session with the HCP of about 15 minutes of effective training. Above all, these visits were scheduled to answer potential questions, informally monitor progress, adjust the amount of support from the orthosis when needed, and encourage participants to practice more when training duration so far was low.

\section{Evaluation}

\section{User acceptance}

The frequency and duration of practice (effective use and excluding calibration or system setup procedures) were recorded automatically by the system and displayed in the user interface. Motivation and usability of the system during training as experienced by the participants was measured using the Intrinsic Motivation Inventory (IMI) and the System Usability Scale (SUS), respectively. The IMI is a questionnaire with several dimensions that provides qualitative information about the content and level of motivation that a participant experiences during an intervention. ${ }^{29,30}$ It is scored on a seven-point Likert scale ranging from 'not at all true' to 'very true'. A neutral score on the IMI is four, and a higher score means a more positive result on motivation.

The SUS is a 10-item scale giving a global view of subjective experience of usability. Questions are scored on a five-point Likert scale ranging from 'strongly agree' to 'strongly disagree'. Scores are translated to $0-100 \%$, with a higher score meaning better usability. ${ }^{31}$ Interventions that score in the 90s are exceptional, scores in the 70 s and 80 s are promising, and with SUS scores below 50 one can be almost certain that the product or intervention 
will have usability difficulties in the field. ${ }^{32,33}$ Both the IMI and SUS were completed during the post-intervention evaluation measurement (T08).

\section{Arm and hand function}

Clinical tests were used to quantify general arm function, before and after training and at two-month follow-up. The scales used are valid, standardized assessments, which were applied according to their specific protocols. The Action Research Arm Test (ARAT) evaluates coordination, dexterity and upper extremity function on four subtests (grasp, grip, pinch, and gross arm movement). The maximum score is 57 points. ${ }^{34-36}$ The upper extremity part of the Brunnstrom Fugl-Meyer assessment (FM) evaluates motor status and the degree of synergy-development in the upper extremity, with a maximum score of $66 .{ }^{37,} 38$ Separate scores were also calculated for proximal (maximum $=42$ points) and distal components of the FM (maximum = 24 points). The Motor Activity Log (MAL) is a semi-structured interview specifically designed for hemiparetic stroke patients. It assesses the perceived use of the paretic arm and hand (amount of use and quality of movement) during activities of daily living. ${ }^{39}$ The maximum score for both subsections is five points. The Stroke Impact Scale (SIS) is a questionnaire which assesses eight domains related to function, activities, and participation. Each domain score has a range of zero to hundred percent. ${ }^{40,41} \mathrm{~A}$ higher score indicates better quality of life after stroke.

\section{Statistical analysis}

Statistical analyses were performed using IBM SPSS Statistics 19 for Windows. All outcomes were inspected for normal distribution using histogram plots including normal curves and normal probability plots, and Shapiro-Wilk tests, prior to selection of appropriate statistical tests. Descriptive statistics (mean with standard deviation (SD) for normal distributed outcomes, or median with interquartile range (IQR) for non-parametric outcomes) were used to describe the participant characteristics and all outcome measures. Clinical variables over time were analyzed using mixed models repeated measures analysis with adjustments for multiple comparisons (Sidak), or the non-parametric equivalent, Friedman's ANOVA. In case of significance for non-parametric variables, additional Wilcoxon Signed Ranks Tests for multiple comparisons were performed, using adjusted P-values in accordance with the Holm-Bonferroni correction. Additionally, the relationship between training duration and training-induced changes was assessed with Pearson's (or non-parametric Spearman's) correlation coefficient. The level for significance was set at $\alpha<0.05$ for all statistical tests. When using the Holm-Bonferroni correction, significance levels corresponded with $\alpha<$ $0.0167,0.025$ and 0.05 (sorted in order of smallest to largest P-value, respectively). 


\section{RESULTS}

\section{Participants}

Twenty-four participants were included in the study. Three participants were lost during the study because of shoulder pain due to external causes, technical problems with the system, and a desire to not continue. No post-training data is available from these participants, and they were not included in data analysis. The characteristics of the remaining 21 participants are displayed in Table 4.1. The group involved 9.5\% mildly, 57.2\% moderately and 33.3\% severely affected stroke patients, based on a categorization of baseline FM score. ${ }^{42}$ Data on the SIS are incomplete for two participants, so analysis for this outcome was performed over 19 participants.

Table 4.1 Participant characteristics at baseline

\begin{tabular}{lc}
\hline & Participants $(\boldsymbol{N}=\mathbf{2 1})$ \\
\hline Sex (male/female) $^{\mathrm{a}}$ & $10 / 11$ \\
Age (years) $^{\mathrm{b}}$ & $59 \pm 13(34-80)$ \\
Time post stroke (months) $^{\mathrm{b}}$ & $19 \pm 14(6-50)$ \\
Type of stroke (infarction/hemorrhage) $^{\mathrm{a}}$ & $19 / 2$ \\
Affected body side (right/left) $^{\mathrm{a}}$ & $7 / 14$ \\
Dominant arm (right/left) $^{\mathrm{a}}$ & $19 / 2$ \\
FM score (maximal 66 points) $^{\mathrm{b}}$ & $33.1 \pm 15.8(9-56)$ \\
ARAT score (maximal 57 points) $^{\mathrm{b}}$ & $25.7 \pm 21.0(3-55)$ \\
\hline
\end{tabular}

Abbreviations: FM score: Fugl-Meyer assessment score at baseline; ARAT Score: Action Research Arm Test score at baseline. ${ }^{a}$ Absolute numbers, ${ }^{b}$ Mean \pm standard deviation (range)

\section{User acceptance}

Figure 4.2 shows the main effects concerning user experience. Twenty-one participants used the system for six weeks, but with a large amount of variation in effective use between and within individuals. Mean training duration for the group, averaged per week over six weeks, was 105 minutes (SD = 66 minutes), whereas training duration ranged from 13 to 284 minutes per week across participants. In general, the motivation during training was positive, as reflected in the mean score on the IMI of 5.2 points (SD $=0.9$ points). The mean score on the SUS is $69 \%$ (SD = 17\%). On individual level, ten participants rated usability over $70 \%$, seven between $50 \%$ and $70 \%$ and four below $50 \%$.

\section{Arm and hand function}

The FM and SIS improved significantly after training ( $P=0.002$ and 0.004 , respectively). Subsequent multiple comparison analysis showed significant improvements between T01 and T08, with a median improvement of 4 points for FM, and mean improvement of 3.7 points for SIS (Table 4.2). For FM, this was sustained at two-month follow-up with a median 
improvement of 3 points from T01 to T15, and showed a trend for SIS (mean improvement of 3.5 points), indicating improved quality of life after stroke. Considering specific SIS domains, the participants improved significantly on the domains strength $(P=0.004)$ and mobility $(P$ $=0.014)$, and showed a trend for ADL $(P=0.077)$ after training. The proximal component of the FM showed a significant improvement over the training period $(P=0.012)$, with a mean improvement of 2.8 points between T01-T08. This was sustained at two-month follow-up (3.1 points improvement overall). A trend was found for the distal component of the FM ( $P$ $=0.078$ ). The other clinical outcomes showed no statistically significant changes after the six weeks of training or at follow-up (Table 4.2).

a)

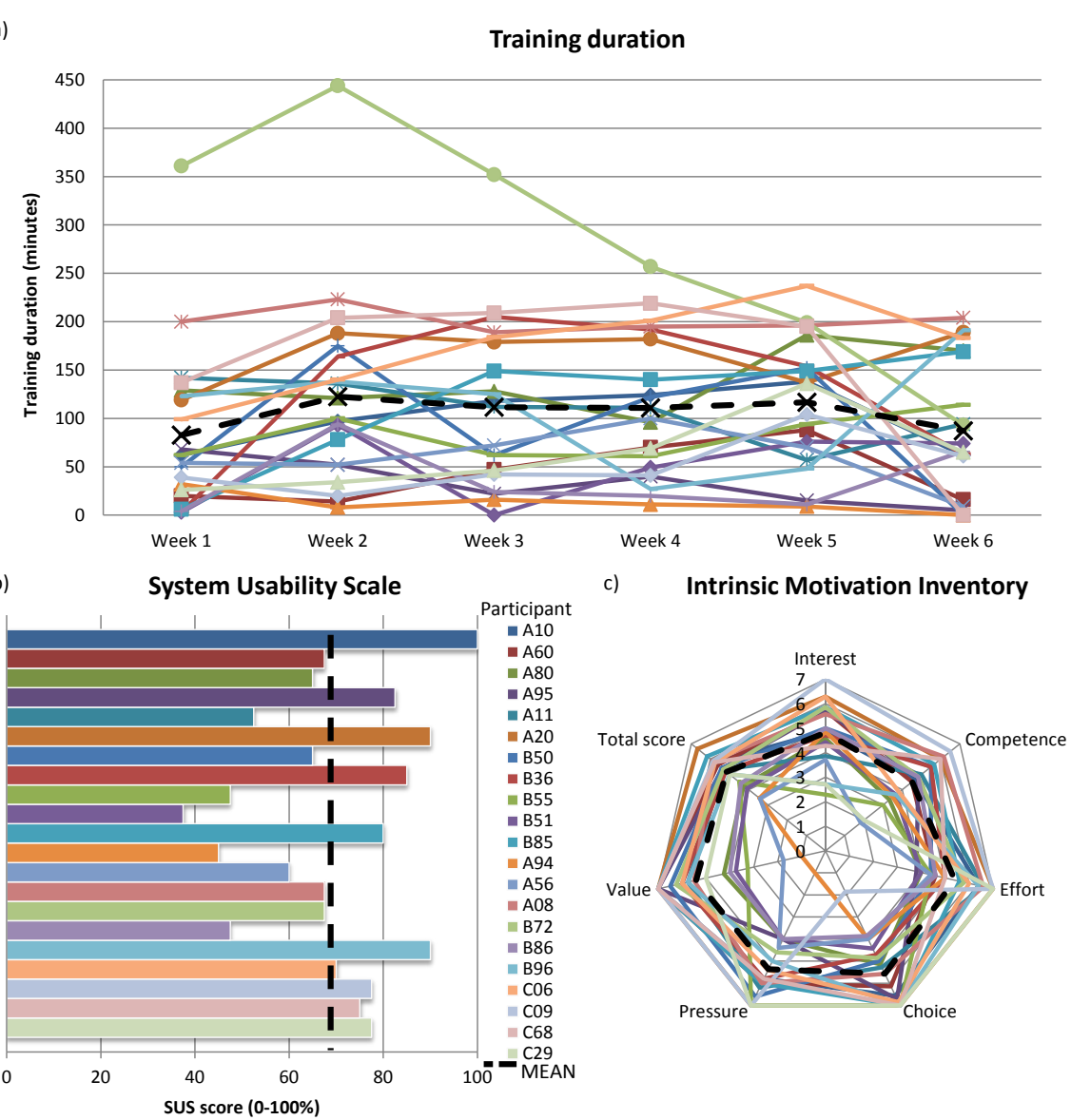

Participant

$\underset{-A 60}{\longrightarrow \text { A } 10}$

$\rightarrow$ A 80

$\longrightarrow$ A95

-A11

+ B50

B36
B55

- B51

$-\mathrm{B} 85$
$-\mathrm{A} 94$

$-\mathrm{A} 56$
$\times$

* A08
$-\mathrm{B} 72$

+ B86

B96
C06

$\longrightarrow$ C09

$-\mathrm{C} 68$

C29

$\rightarrow-$ MEAN

b)

Participant

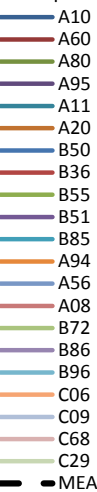

Figure 4.2 Individual results (colored lines) with group averages (dotted line) on user acceptance for (a) Training duration per week (b) System Usability Scale and (c) Intrinsic Motivation Inventory 
Table 4.2 Clinical outcome scores pre-training (T01), post-training (T08) and at follow-up (T15)

\begin{tabular}{|c|c|c|c|c|c|c|c|}
\hline Outcome measures & P-values & T01 & T08 & T15 & $\Delta \mathrm{T} 08-\mathrm{T} 01$ & $\Delta \mathrm{T} 15-\mathrm{T} 01$ & $\Delta \mathrm{T} 15-\mathrm{T} 08$ \\
\hline \multirow[t]{2}{*}{$\mathrm{FM}^{\mathrm{b}}$} & & $37(30)$ & $41(32)$ & $40(32)$ & $+4(6)$ & $+3.0(9)$ & $+0(4)$ \\
\hline & $P=0.002$ & & & & 0.001 & 0.003 & 0.312 \\
\hline \multirow[t]{2}{*}{ FM_prox ${ }^{a}$} & & $21.7(8.2)$ & $24.5(8.8)$ & $24.8(9.0)$ & $+2.8(3.9)$ & $+3.1(4.4)$ & $+0.3(1.5)$ \\
\hline & $P=0.012$ & & & & 0.010 & 0.012 & 0.784 \\
\hline \multirow[t]{2}{*}{ FM_dist ${ }^{b}$} & & $15(17)$ & $16(17)$ & $16(18)$ & $+1(2)$ & $+1(4)$ & $+0(1)$ \\
\hline & $P=0.078$ & & & & & & \\
\hline \multirow[t]{2}{*}{ ARAT $^{\mathrm{b}}$} & & $20(44)$ & $29(45)$ & $29(45)$ & $+1(2)$ & $+1(4)$ & $+0(3)$ \\
\hline & $P=0.101$ & & & & & & \\
\hline \multirow[t]{2}{*}{ MAL_AOU ${ }^{b}$} & & $0.7(2.0)$ & $0.6(2.2)$ & 1.5 (1.9) & $+0.0(0.5)$ & $+0.0(0.8)$ & $+0.0(0.4)$ \\
\hline & $P=0.753$ & & & & & & \\
\hline \multirow[t]{2}{*}{ MAL_QOM ${ }^{b}$} & & $0.9(1.8)$ & $1.1(1.8)$ & $1.5(1.8)$ & $+0.0(0.6)$ & $+0.2(0.5)$ & $+0.1(0.4)$ \\
\hline & $P=0.229$ & & & & & & \\
\hline \multirow[t]{2}{*}{ SIS $^{\mathrm{a}}$} & & $56.3(13.2)$ & $60.0(13.9)$ & $59.8(15.2)$ & $3.7(4.9)$ & $3.5(6.6)$ & $-0.1(4.1)$ \\
\hline & $P=0.004$ & & & & 0.003 & 0.055 & 0.999 \\
\hline \multirow[t]{2}{*}{ SIS_Strength ${ }^{a}$} & & $44.7(20.4)$ & $55.4(21.7)$ & $49.4(22.0)$ & $+10.6(11.8)$ & $+4.7(13.6)$ & $-5.9(15.0)$ \\
\hline & $P=0.004$ & & & & 0.003 & 0.352 & 0.169 \\
\hline \multirow[t]{2}{*}{ SIS_Memory ${ }^{\mathrm{b}, \mathrm{c}}$} & & $90.6(46.9)$ & $87.5(31.3)$ & $87.5(34.4)$ & $+0.0(3.1)$ & $+0.0(6.3)$ & $+0.0(6.3)$ \\
\hline & $P=0.303$ & & & & & & \\
\hline \multirow[t]{2}{*}{ SIS_Emotion ${ }^{a}$} & & $65.7(15.9)$ & $67.6(15.9)$ & $70.0(16.3)$ & $+1.9(11.0)$ & $+4.4(12.0)$ & $+2.5(11.1)$ \\
\hline & $P=0.248$ & & & & & & \\
\hline \multirow[t]{2}{*}{ SIS_Communication ${ }^{\mathrm{b}, \mathrm{c}}$} & & 85.7 (17.9) & $89.3(14.3)$ & $85.7(14.3)$ & $+0.0(14.3)$ & $+0.0(14.3)$ & $+0.0(10.7$ \\
\hline & $P=0.523$ & & & & & & \\
\hline \multirow[t]{2}{*}{ SIS_ADL ${ }^{a}$} & & $57.1(18.9)$ & $59.3(20.6)$ & $54.6(19.8)$ & $+2.2(7.4)$ & $-2.5(9.2)$ & $-4.7(9.9)$ \\
\hline & $P=0.077$ & & & & & & \\
\hline \multirow[t]{2}{*}{ SIS_Mobility ${ }^{b, c}$} & & $55.0(40.0)$ & $67.5(42.5)$ & $70.0(40.0)$ & $+2.5(12.5)$ & $+5.0(15.6)$ & $+0.0(15.0)$ \\
\hline & $P=0.014$ & & & & 0.051 & 0.029 & 0.955 \\
\hline \multirow[t]{2}{*}{ SIS_Hand_function ${ }^{\mathrm{b}, \mathrm{c}}$} & & $5.0(55.0)$ & $15.0(60.0)$ & $20.0(55.0)$ & $+0.0(15.0)$ & $+0.0(15.0)$ & $+0.0(10.0)$ \\
\hline & $P=0.927$ & & & & & & \\
\hline \multirow[t]{2}{*}{ SIS_Participation ${ }^{a}$} & & $48.9(20.8)$ & $50.9(21.1)$ & $56.0(23.3)$ & $+2.1(16.0)$ & $+7.2(17.0)$ & $+5.1(13.9)$ \\
\hline & $P=0.132$ & & & & & & \\
\hline \multirow[t]{2}{*}{ SIS_Recovery ${ }^{\mathrm{a}}$} & & $52.3(17.7)$ & $54.0(20.0)$ & $53.0(24.8)$ & $+1.7(11.3)$ & $+0.8(17.7)$ & $+1.0(14.8)$ \\
\hline & $P=0.813$ & & & & & & \\
\hline
\end{tabular}

Group mean or median values at T01, T08 and T15 are displayed, in addition to change scores over training ( $\triangle \mathrm{T} 08-\mathrm{T} 01)$, after the end of training ( $\triangle \mathrm{T} 15-\mathrm{T} 08)$ and over training and follow-up in total ( $\Delta \mathrm{T} 15-\mathrm{T} 01)$. P-values of the main effect are displayed in the first column and in case of significance, P-values of post-hoc tests are displayed in the last three columns (significant P-values in bold). Abbreviations: FM: Fugl-Meyer, FM_prox: Fugl-Meyer proximal part, FM_dist: Fugl-Meyer distal part, ARAT: Action Research Arm Test, MAL_AOU: Motor Activity Log Amount of Use, MAL_QOM: Motor Activity Log Quality of Movement, SIS: Stroke Impact Scale, SIS_ADL: Stroke Impact Scale Activities of Daily Living section, T01: baseline measurement pre-training, T08: evaluation measurement post-training, T15: two-month follow-up evaluation measurement. $\quad{ }^{a}=$ Normally distributed variables displayed by mean (standard deviation) and analyzed by mixed models repeated measures analysis. ${ }^{b}=$ Non-parametric variables displayed by median (interquartile range) and analyzed by Friedman ANOVA. ${ }^{c}=N=19$ because of incomplete dataset 
Examination of the individual scores of each participant (Figure 4.3) shows that some participants showed substantial improvements on clinical outcomes. Eight of the 21 participants reached minimal clinically important differences (MCID) for FM of $10 \%$ improvement. ${ }^{43}$ ARAT scores showed less marked improvements, with three participants reaching the MCID of $10 \%$ improvement. ${ }^{43}$ Seventeen of the 21 participants reported improvements in quality of movement of the affected arm in daily life on the MAL, of which 14 also reported improved amount of use. Of those, seven achieved MCID improvements of 0.5 points ${ }^{44}$ for amount of use, and five for quality of movement on the MAL.

When examining the relationship between clinical outcome changes and training duration, a correlation was observed for dexterity. A moderate-strong, significant correlation was found between ARAT changes and training duration (Spearman's rho $=0.686, P=0.001$ ) (Figure 4.4), which means that a higher training duration is associated with a larger improvement in arm and hand dexterity.

\section{Discussion}

The current study is one of the first studies in which technology-supported arm and hand training is performed in the patient's home, where participants independently used a training device for both the proximal and distal arm and hand, which physically interacted with the participants, without direct supervision of an HCP. The objective was to examine feasibility (in terms of user acceptance and effective use) and potential clinical changes of the use of the training system. Results showed high marginal usability with potential for application in the field and motivation during training was good, which was reflected in a fair amount of effective use of 105 minutes per week. In addition, arm function improved over training and was sustained at two-month follow-up. Taking into account cost estimates as calculated within the SCRIPT project, ${ }^{27}$ home training with indirect supervision seems not only to have clinical value, but could also be economically viable in comparison with conventional rehabilitation and even home training as applied in many research studies nowadays. Within one year, this technology-supported training for six weeks per patient can be more cost-efficient when compared to dose-matched conventional therapy in the clinic with direct supervision by a therapist. Remotely monitored and physically supported arm and hand training at home showing feasibility and potential clinical value implies that an approach without direct, online therapist supervision should have the potential to allow practice without dependence on therapist availability. This might enable increased dose of training with respect to supervised treatment in clinical settings. The results obtained in our study correspond with those found in another home-based study, recently published by Sivan et al. ${ }^{45}$ They evaluated the feasibility of a robotic device for proximal arm training that can be used independently at home by stroke survivors with upper limb weakness. 
a)

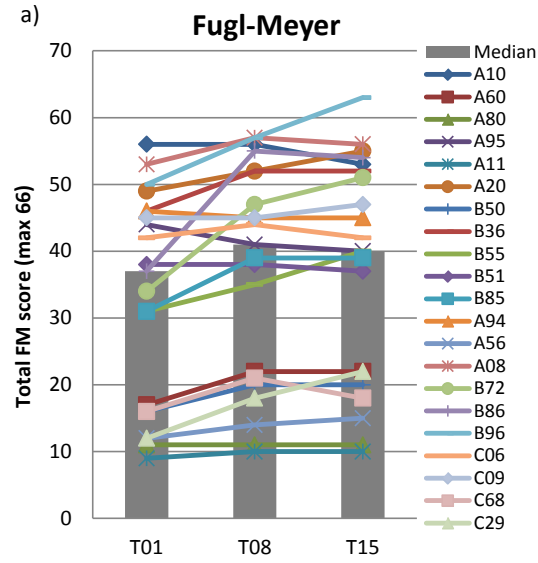

c)

Motor Activity Log Amount of Use

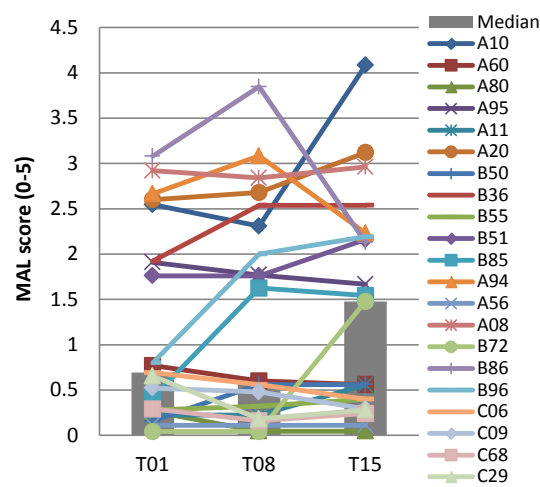

b) Action Research Arm Test

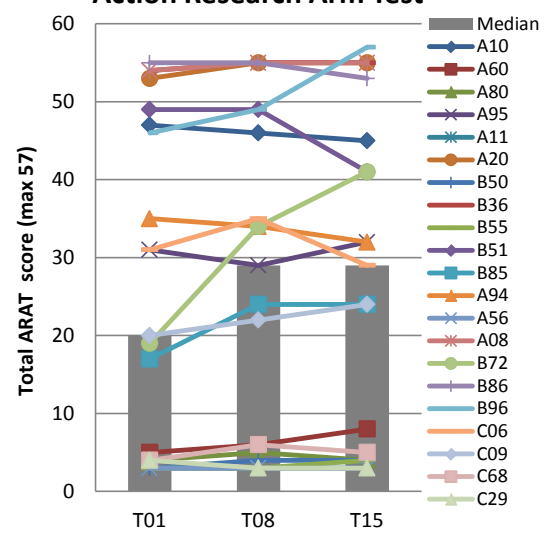

d) Motor Activity Log Quality of Movement

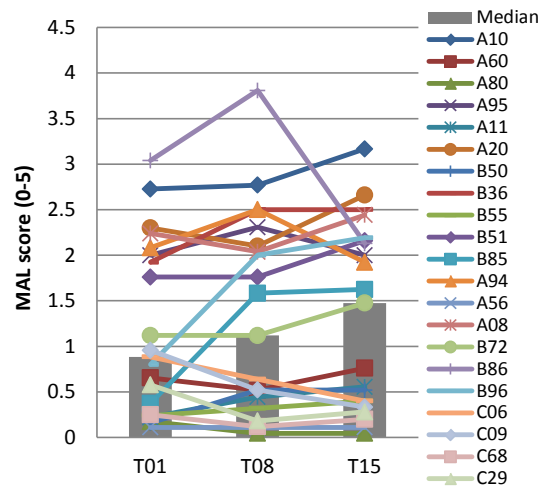

e) Stroke Impact Scale *

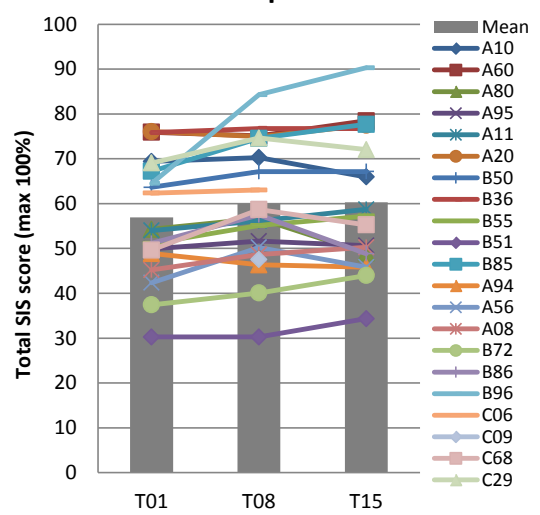

Figure 4.3 Individual (colored lines) and group (grey bars) results of the clinical scales for (a) Fugl-Meyer assessment, (b) Action Research Arm Test, (c) Motor Activity Log Amount of Use, (d) Motor Activity Log Quality of Movement and (e) Stroke Impact Scale. Abbreviations: FM: Fugl-Meyer, ARAT: Action Research Arm Test, SIS: Stroke Impact Scale, MAL: Motor Activity Log, T01: baseline measurement pre-training, T08: evaluation measurement post-training, T15: two-month follow-up evaluation measurement. *Missing data Stroke Impact Scale: T01 $N=20$, T08 $N=21$, and T15 $N=19$ 


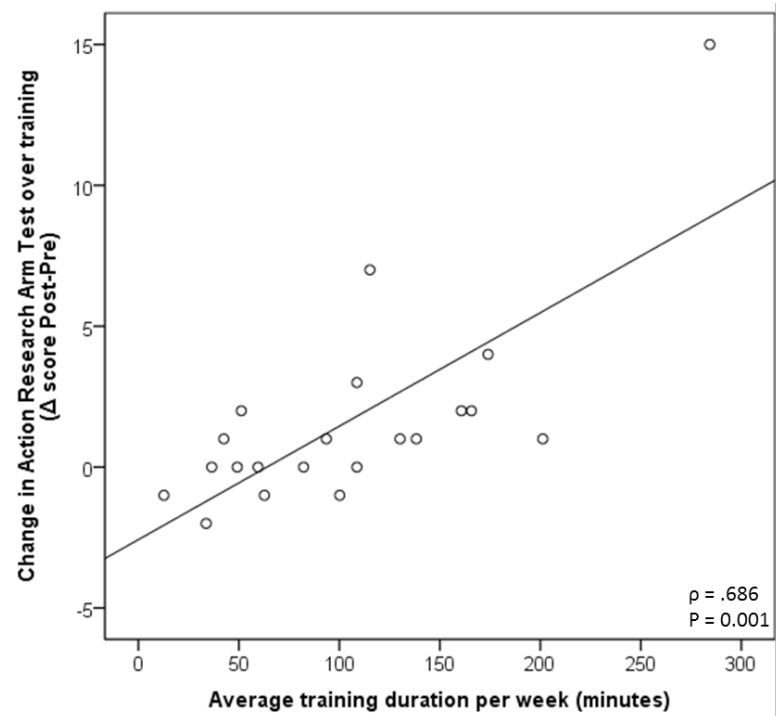

Figure 4.4 Scatter plot of average training duration and changes in Action Research Arm Test score over training

On group level, they found a mean training duration of 520 minutes during the 8-week study (which is about 65 minutes per week), and improvements on FM and ARAT of 1 point and 3 points, respectively. These improvements are comparable to the findings reported here, although these were slightly less pronounced on function level compared to the present study. ${ }^{45}$

A review by Coupar et al. ${ }^{46}$ involved four studies of in-home telerehabilitation for the upper limb after stroke. They found home-based upper limb programs to be no more or no less effective for arm motor impairment outcomes compared to upper limb programs conducted in hospitals. ${ }^{46}$ In all included studies, the patients were (remotely) supervised at a fixed time, and without examining the effective amount of self-administered training at home. This is similar to other studies, in which a therapist in the clinic conducted treatment sessions with patients located at home, ${ }^{47}$ or in a different room in the clinic. ${ }^{48,49}$ In those studies the training sessions were scheduled beforehand, with about five hours (or more) practice per week. In our study, the HCP visited the participants only once per week (of which maximal 15 minutes of effective training), and patients were able to make their own decisions about their training schedule, without further direct real-time supervision of a therapist. The rationale for this was to remove the training constraints, and increase therapy availability. However, this makes it difficult to compare the effective amount of training in the abovementioned studies with our study. The achieved training duration of approximately 105 minutes per week (about 15 minutes per day on average) suggests that stroke patients do have the incentive to train independently at home. When comparing specifically with recent 
studies into home-based self-administered upper limb therapy programs after stroke, the presently recorded effective use equals or even exceeds the adherence in those studies. ${ }^{45}$, 50,51 It is important to note that this 15 minutes of training stored in the portal involved only effective training time, which does not take into account for example donning and doffing of the orthosis and the calibration procedure. The total time participants spent on the selfadministered training in this study would therefore be higher.

Usability of the system was rated sufficient, but the system might need improvements, which will be taken into account for further development of the system. When the technology has matured more, this should be tested in a large cohort study or cohort multiple randomized controlled trial as the next stage in telemedicine evaluation, ${ }^{26}$ to investigate clinical effects and explore if the results found here are comparable to conventional rehabilitation. Products or interventions with SUS scores below $70 \%$ should be considered a candidate for increased scrutiny and continued improvement. ${ }^{33}$ On individual level, half of the participants rated usability over $70 \%$, which means that the technology will have good to excellent chances for acceptance in the field, whereas only four participants scored SUS below $50 \%$ (a product or intervention will probably have usability difficulties). ${ }^{32,33}$ One of the examples of usability issues encountered by participants was unstable recording of the arm movements in space, as measured by an inertial sensor, which did not allow participants to fluently control and play one of the games involving reaching movement. Remarkably, two of the four participants with low scores on usability still performed a fair amount of training per week (>80 minutes). These two participants were not accustomed to new technologies and computers and found it frustrating to interact with the orthosis, computer and games. Despite their initial reservations, they decided to continue the therapy. Moreover, the positive results on IMI on group level indicate that participants perceived the training to be interesting and they enjoyed engagement with the system, which emphasizes the potential of this type of training for involving stroke patients actively in (prolonged) training.

Although a direct comparison between self-administered home training and a control group receiving comparable conventional treatment is lacking in the present study, the improvements in motor function of the arm in the present study correspond with those found in other robot-aided studies in chronic stroke in a clinical setting, ${ }^{14,15,17}$ and with therapy programs for the upper limb performed at home. ${ }^{10,45,46}$ The improvements in our study involved particularly proximal arm movements. It is conceivable that reduction of arm weight did contribute to the gains presented by the proximal part of the FM, which is similar to results presented in previous studies. ${ }^{52,53}$ On individual level, eight participants (three mildly, four moderately, one severely impaired participant) achieved MCID of $10 \%$ improvement for FM. Remarkably, seven of the 21 participants reached MCID of MAL, indicating that a third of the patients perceived a better use of the arm in daily activities, 
even though this was not reflected in an improved capacity as measured by ARAT. Of these seven patients, three were moderately and four mildly impaired. Hence, it seems that mild to moderate impaired patients benefit most from the intervention, which corresponds with previously reported robot-aided upper limb exercise training after stroke. ${ }^{54}$ Furthermore, it is important to keep in mind that this study was conducted with chronic stroke patients. Ideally, home-based training should be considered at an earlier stage, for example as soon as inpatient rehabilitation is finished. This likely involves patients in the (sub)acute phase as well, where larger treatment effects would be expected. Although some participants did achieve clinically relevant gains in arm and hand function, others did not, underlining the need for further examination of which factors (for example age, time post stroke, stroke severity, training adherence, personal characteristics etc.) are associated with better treatment outcomes. This will assist in identifying who would benefit most from technologysupported, self-administered training at home, and ultimately, to find out for which patients this kind of home-based training is most suitable.

Many training studies using technology supported devices focus on either the proximal ${ }^{14}$, ${ }^{54}$ or distal ${ }^{21,55}$ arm only. Important aspects of the current study are that it aimed to involve functional movements of both the proximal and distal arm and hand within motivating rehabilitation games during training. The games available for practice mostly required movements of the arm, wrist and hand in sequence, like reaching, followed by wrist flexion or extension, and then hand opening and closing. This may have played a role in the limited effect on activity level, because proximal and distal movements may not have been integrated optimally. Although the games specifically incorporated hand gestures, these were rather coarse with generic flexion and extension of the thumb and fingers, and they did not contain specific functional grasps representing the handling of various objects. The expectation is that when such aspects are incorporated more specifically, exercises become even more functional and task-specific, which is likely to further enhance the clinical impact, predominantly on activity level.22, 56 Therefore, integration of proximal and distal arm movements simultaneously, together with more functional and a larger variety of grasps, should be considered more specifically when designing games or exercises with a diversity of complexity for application in a training system dedicated to self-administered practice.

Although the arm function improvements in this study were comparable to those achieved through other robot-aided studies, they are still modest. An important factor might be the effective training duration. Although the average training duration was promising with approximately 105 minutes per week, especially in the light of effective use in other research into self-administered training, ${ }^{45,50,51}$ strong interindividual differences were observed. Qualitative information obtained during home visits revealed that some of the patients returned back to work or had a busy daytime schedule, limiting the time they had 
for the training. It is known that dose of robot-assisted training ${ }^{18,57}$ and conventional stroke rehabilitation ${ }^{4,58}$ is an important factor for clinical improvement, although an optimal or minimal dose is not yet known. The advised dose of 30 minutes per day, 6 days per week in this study (which totals to 18 hours in six weeks) corresponds closely with the 16 hours of additional training recommended by Kwakkel et al. to achieve clinically relevant functional improvements..$^{59}$ Observations on individual cases concerning a higher training duration and clinical improvements suggest that if an increase in training duration can be established, more pronounced clinical improvements might be achieved. Six participants who showed marked improvements in FM score over training had a rather high training duration $(>100$ minutes per week), which was also supported by the significant correlation between training duration and improvements in dexterity. In addition, the incorporation of a high variety of games probably enhances motivation during training, which might further stimulate a higher effective training duration. Additional motivational strategies might be implemented to increase participants' effective training time during self-administered training even more. Future studies might consider approaches from the field of psychology to further explore this potential.

\section{CONCLUSIONS}

In this study we evaluated the feasibility and potential clinical changes of self-administered and remotely monitored arm and hand training at home, with physical support from a dynamic wrist and hand orthosis and games representing exercises, in chronic stroke. Usability was perceived as sufficient and motivation was good, although issues were identified that need further improvements. Together with an effective use of 105 minutes per week, these findings indicate that home-based arm and hand training with physical support from a dynamic orthosis is a feasible tool to enable self-administered practice at home. Arm function improved, together with modest improvements in quality of life, indicating home-based arm and hand training can have clinical value, especially for mild to moderately impaired patients. By stressing the functional nature of the exercises even more in future applications, results on activity level may become more pronounced. Future research using a larger sample of participants including a control group should further examine ways to stimulate effective use and explore which factors are associated with better treatment outcomes, to identify those who would benefit most from this remotely supervised technology-supported training at home.

\section{REFERENCES}

1. Kwakkel G, Kollen BJ, van der Grond J and Prevo AJ. Probability of regaining dexterity in the flaccid upper limb: impact of severity of paresis and time since onset in acute stroke. Stroke. 2003; 34: 2181-6.

2. Schaechter JD. Motor rehabilitation and brain plasticity after hemiparetic stroke. Progress in Neurobiology. 2004; 73: 61-72. 
3. Krakauer JW. Arm function after stroke: from physiology to recovery. Semin Neurol. 2005; 25: 384-95.

4. Kleim JA and Jones TA. Principles of experience-dependent neural plasticity: implications for rehabilitation after brain damage. J Speech Lang Hear Res. 2008; 51: S225-39.

5. Fisher BE and Sullivan KJ. Activity-dependent factors affecting poststroke functional outcomes. Top Stroke Rehabil. 2001; 8: 31-44.

6. Hermens HJ and Vollenbroek-Hutten MMR. Towards remote monitoring and remotely supervised training. Journal of Electromyography and Kinesiology. 2008; 18: 908-19.

7. Lupton D and Seymour W. Technology, selfhood and physical disability. Soc Sci Med. 2000; 50: 1851-62.

8. Burdea GC. Virtual rehabilitation--benefits and challenges. Methods Inf Med. 2003; 42: 519-23.

9. Vugts CJ, van der Velden LFJ, Hingstman L, (NIVEL), van der Velde F and van der Windt W, (Prismant). Behoefteraming fysiotherapeuten 2002-2015. Utrecht: Nivel (http:// www.nivel.nl), 2003.

10. Johansson T and Wild C. Telerehabilitation in stroke care--a systematic review. J Telemed Telecare. 2011; 17: 1-6.

11. Laver KE, Schoene D, Crotty M, George S, Lannin NA and Sherrington C. Telerehabilitation services for stroke. Cochrane Database Syst Rev. 2013; 12: CD010255.

12. Fasoli SE, Krebs $\mathrm{HI}$ and Hogan N. Robotic technology and stroke rehabilitation: translating research into practice. Top Stroke Rehabil. 2004; 11: 11-9.

13. Maciejasz P, Eschweiler J, Gerlach-Hahn K, Jansen-Troy A and Leonhardt S. A survey on robotic devices for upper limb rehabilitation. Journal of Neuroengineering and Rehabilitation. 2014; 11: 3.

14. Prange GB, Jannink MJ, Groothuis-Oudshoorn CG, Hermens HJ and Ijzerman MJ. Systematic review of the effect of robot-aided therapy on recovery of the hemiparetic arm after stroke. J Rehabil Res Dev. 2006; 43: 171-84.

15. Kwakkel G, Kollen BJ and Krebs HI. Effects of robot-assisted therapy on upper limb recovery after stroke: a systematic review. Neurorehabil Neural Repair. 2008; 22: 11121.

16. Mehrholz J, Pohl M, Platz T, Kugler J and Elsner B. Electromechanical and robot-assisted arm training for improving activities of daily living, arm function, and arm muscle strength after stroke. Cochrane Database Syst Rev. 2015; 11: CD006876.

17. Basteris A, Nijenhuis SM, Stienen AH, Buurke JH, Prange GB and Amirabdollahian F. Training modalities in robot-mediated upper limb rehabilitation in stroke: a framework for classification based on a systematic review. J Neuroeng Rehabil. 2014; 11: 111.

18. Lo AC, Guarino PD, Richards LG, et al. Robot-assisted therapy for long-term upper-limb impairment after stroke. N Engl J Med. 2010; 362: 1772-83.

19. Wagner TH, Lo AC, Peduzzi P, et al. An economic analysis of robot-assisted therapy for long-term upper-limb impairment after stroke. Stroke. 2011; 42: 2630-2.

20. Klamroth-Marganska V, Blanco J, Campen K, et al. Three-dimensional, task-specific robot therapy of the arm after stroke: a multicentre, parallel-group randomised trial. The Lancet Neurology. 2014; 13: 159-66.

21. Balasubramanian S, Klein J and Burdet E. Robot-assisted rehabilitation of hand function. Curr Opin Neurol. 2010; 23: 661-70.

22. Timmermans AA, Seelen HA, Willmann RD and Kingma H. Technology-assisted training of arm-hand skills in stroke: concepts on reacquisition of motor control and therapist guidelines for rehabilitation technology design. J Neuroeng Rehabil. 2009; 6: 1.

23. Oujamaa L, Relave I, Froger J, Mottet D and Pelissier JY. Rehabilitation of arm function 
after stroke. Literature review. Ann Phys Rehabil Med. 2009; 52: 269-93.

24. Wisneski KJ and Johnson MJ. Quantifying kinematics of purposeful movements to real, imagined, or absent functional objects: implications for modelling trajectories for robot-assisted ADL tasks. Journal of Neuroengineering and Rehabilitation. 2007; 4: 7.

25. Loureiro RC, Harwin WS, Lamperd R and Collin C. Evaluation of reach and grasp robotassisted therapy suggests similar functional recovery patterns on proximal and distal arm segments in sub-acute hemiplegia. IEEE transactions on neural systems and rehabilitation engineering : a publication of the IEEE Engineering in Medicine and Biology Society. 2014; 22: 593-602.

26. Jansen-Kosterink SM. The added value of telemedicine services for physical rehabilitation. Enschede: University of Twente, 2014, p. 155.

27. Amirabdollahian F, Ates S, Basteris A, et al. Design, development and deployment of a hand/wrist exoskeleton for home-based rehabilitation after stroke - SCRIPT project. Robotica. 2014; 32: 1331-46.

28. Ates S, Lobo-Prat J, Lammertse P, van der Kooij H and Stienen AH. SCRIPT Passive Orthosis: Design and technical evaluation of the wrist and hand orthosis for rehabilitation training at home. IEEE International Conference on Rehabilitation Robotics. 2013; 2013: 1-6.

29. Ryan RM. Control and information in the intrapersonal sphere: An extension of cognitive evaluation theory. Journal of Personality and Social Psychology. 1982; 43: 450-61.

30. McAuley E, Duncan T and Tammen VV. Psychometric properties of the Intrinsic Motivation Inventory in a competitive sport setting: a confirmatory factor analysis. Res Q Exerc Sport. 1989; 60: 48-58.

31. Brooke J. SUS: A Quick and Dirty Usability Scale. In: Jordan PW, Thomas B, Weerdmeester BA and McClelland IL, (eds.). Usability Evaluation in Industry. London: Taylor \& Francis., 1996.

32. Bangor A, Kortum P and Miller J. Determining what individual SUS scores mean: Adding an adjective rating scale. Journal of usability studies. 2009; 4: 114-23.

33. Bangor A, Kortum PT and Miller JT. An Empirical Evaluation of the System Usability Scale. International Journal of Human-Computer Interaction. 2008; 24: 574-94.

34. Lyle RC. A performance test for assessment of upper limb function in physical rehabilitation treatment and research. Int J Rehabil Res. 1981; 4: 483-92.

35. Carroll D. A Quantitative Test of Upper Extremity Function. J Chronic Dis. 1965; 18: 47991.

36. Yozbatiran N, Der-Yeghiaian L and Cramer SC. A standardized approach to performing the action research arm test. Neurorehabil Neural Repair. 2008; 22: 78-90.

37. Fugl-Meyer AR, Jaasko L, Leyman I, Olsson S and Steglind S. The post-stroke hemiplegic patient. 1. A method for evaluation of physical performance. Scand J Rehabil Med. 1975; 7: 13-31.

38. Deakin A, Hill H and Pomeroy VM. Rough Guide to the Fugl-Meyer Assessment: Upper limb section. Physiotherapy. 2003; 89: 751-63.

39. van der Lee JH, Beckerman $\mathrm{H}$, Knol DL, de Vet $\mathrm{HC}$ and Bouter LM. Clinimetric properties of the motor activity log for the assessment of arm use in hemiparetic patients. Stroke. 2004; 35: 1410-4.

40. van de Port IGL, Leendes K, Sellmeijer D, Zuidgeest A and Kwakkel G. Betrouwbaarheids en concurrente validiteit van de Nederlandse Stroke Impact Scale 2.0 bij patienten met een CVA. Ned Tijdschrift Fysiotherapie. 2008; 118: 12-8.

41. Duncan PW, Wallace D, Lai SM, Johnson D, Embretson S and Laster LJ. The stroke impact scale version 2.0. Evaluation of reliability, validity, and sensitivity to change. Stroke. 1999; 30: 2131-40.

42. Luft AR, McCombe-Waller S, Whitall J, et al. Repetitive bilateral arm training and motor 
cortex activation in chronic stroke: a randomized controlled trial. JAMA. 2004; 292: 1853-61.

43. van der Lee $\mathrm{JH}$, Beckerman $\mathrm{H}$, Lankhorst $\mathrm{GJ}$ and Bouter LM. The responsiveness of the Action Research Arm test and the Fugl-Meyer Assessment scale in chronic stroke patients. J Rehabil Med. 2001; 33: 110-3.

44. van der Lee JH, Wagenaar RC, Lankhorst GJ, Vogelaar TW, Deville WL and Bouter LM. Forced use of the upper extremity in chronic stroke patients: results from a single-blind randomized clinical trial. Stroke. 1999; 30: 2369-75.

45. Sivan M, Gallagher J, Makower S, et al. Home-based Computer Assisted Arm Rehabilitation (hCAAR) robotic device for upper limb exercise after stroke: results of a feasibility study in home setting. Journal of Neuroengineering and Rehabilitation. 2014; 11: 163.

46. Coupar F, Pollock A, Legg LA, Sackley C and van Vliet P. Home-based therapy programmes for upper limb functional recovery following stroke. Cochrane Database Syst Rev. 2012; 5: CD006755.

47. Holden MK, DyarTA, Schwamm Land Bizzi E. Virtual-environment-based telerehabilitation in patients with stroke. Presence: Teleoperators and Virtual Environments. 2005; 14: 214-33.

48. Taub E, Lum PS, Hardin P, Mark VW and Uswatte G. AutoCITE: automated delivery of CI therapy with reduced effort by therapists. Stroke. 2005; 36: 1301-4.

49. Lum PS, Taub E, Schwandt D, Postman M, Hardin P and Uswatte G. Automated Constraint-Induced Therapy Extension (AutoCITE) for movement deficits after stroke. $J$ Rehabil Res Dev. 2004; 41: 249-58.

50. Donoso Brown EV, McCoy SW, Fechko AS, Price R, Gilbertson T and Moritz CT. Preliminary investigation of an electromyography-controlled video game as a home program for persons in the chronic phase of stroke recovery. Arch Phys Med Rehabil. 2014; 95: 14619.

51. Standen PJ, Threapleton K, Connell L, et al. Patients' Use of a Home-Based Virtual Reality System to Provide Rehabilitation of the Upper Limb Following Stroke. Phys Ther. 2014; 11: 11.

52. Amirabdollahian F, Loureiro R, Gradwell E, Collin C, Harwin W and Johnson G. Multivariate analysis of the Fugl-Meyer outcome measures assessing the effectiveness of GENTLE/S robot-mediated stroke therapy. J Neuroeng Rehabil. 2007; 4: 4.

53. Prange GB, Kottink Al, Buurke JH, et al. The Effect of Arm Support Combined With Rehabilitation Games on Upper-Extremity Function in Subacute Stroke: A Randomized Controlled Trial. Neurorehabil Neural Repair. 2015; 29: 174-82.

54. Stein J, Krebs HI, Frontera WR, Fasoli SE, Hughes R and Hogan N. Comparison of two techniques of robot-aided upper limb exercise training after stroke. Am J Phys Med Rehabil. 2004; 83: 720-8.

55. Lum PS, Godfrey SB, Brokaw EB, Holley RJ and Nichols D. Robotic approaches for rehabilitation of hand function after stroke. American journal of physical medicine \& rehabilitation / Association of Academic Physiatrists. 2012; 91: S242-54.

56. Van Peppen RP, Kwakkel G, Wood-Dauphinee S, Hendriks HJ, Van der Wees PJ and Dekker J. The impact of physical therapy on functional outcomes after stroke: what's the evidence? Clin Rehabil. 2004; 18: 833-62.

57. Burgar CG, Lum PS, Scremin AM, et al. Robot-assisted upper-limb therapy in acute rehabilitation setting following stroke: Department of Veterans Affairs multisite clinical trial. J Rehabil Res Dev. 2011; 48: 445-58.

58. Kwakkel G. Impact of intensity of practice after stroke: issues for consideration. Disabil Rehabil. 2006; 28: 823-30. 
Feasibility of post-stroke arm and hand training at home 1

59. Kwakkel G, van Peppen R, Wagenaar RC, et al. Effects of augmented exercise therapy time after stroke: a meta-analysis. Stroke. 2004; 35: 2529-39. 



\section{Chapter 5}

Effects of training with a passive hand orthosis and games at home in chronic stroke: a pilot randomised controlled trial 


\begin{abstract}
Objectives: To compare user acceptance and arm and hand function changes after technology-supported training at home with conventional exercises in chronic stroke. Secondly, to investigate the relation between training duration and clinical changes.
\end{abstract}

Design: A randomized controlled trial.

Setting: Training at home, evaluation at research institute.

Subjects: Twenty chronic stroke patients with severely to mildly impaired arm and hand function.

Interventions: Participants were randomly assigned to six weeks (30 minutes per day, six days a week) of self-administered home-based arm and hand training using either a passive dynamic wrist and hand orthosis combined with computerized gaming exercises (experimental group) or prescribed conventional exercises from an exercise book (control group).

Main measures: Main outcome measures are the training duration for user acceptance and the Action Research Arm Test for arm and hand function. Secondary outcomes are the Intrinsic Motivation Inventory, Fugl-Meyer assessment, Motor Activity Log, Stroke Impact Scale and grip strength.

Results: The control group reported a higher training duration (189 versus 118 minutes per week, $P=0.025)$. Perceived motivation was positive and equal between groups $(P=0.935)$. No differences in clinical outcomes over training between groups were found $(P \geq 0.165)$. Changes in Box and Block Test correlated positively with training duration $(P=0.001)$.

Conclusions: Both interventions were accepted. An additional benefit of technologysupported arm and hand training over conventional arm and hand exercises at home was not demonstrated. Training duration in itself is a major contributor to arm and hand function improvements. 


\section{INTRODUCTION}

To enable intensive rehabilitation for the upper limb after stroke, many technological devices have been developed. ${ }^{1}$ Robotic therapy has been reported to be effective for improving hemiparetic arm function. ${ }^{2-4}$ Nevertheless, the effectiveness of robotic therapy over conventional therapy is questionable and a lack of evidence for improvements on activity level exists. ${ }^{1,5}$ Attention for wrist and hand training in addition to the proximal arm is important to emphasize the functional nature of robotic training and consequently enhance functional gains. ${ }^{6}$ Moreover, improvements after distal robotic therapy have shown generalization effects to the entire upper limb. ${ }^{7}$

To address training intensity, providing functional training at home may enable selfadministered practice, independent from therapists' availability. The majority of devices are designed for the clinical setting. They are technically advanced but consequently too complex to be used by patients independently at home. ${ }^{1,7}$ A home-based training system supporting wrist and hand exercises was developed within the SCRIPT project ${ }^{8}$ and has shown feasibility in chronic stroke patients across the United Kingdom, Italy and the Netherlands. ${ }^{9}$

The objective of the current study was to compare user acceptance, in terms of actual training duration and motivation, and arm and hand function changes after technologysupported training at home to a conventional home exercise program in a small group of chronic stroke patients. A secondary aim of the study was to investigate potential relations between training duration and clinical changes.

\section{METHODS}

\section{Participants}

Participants were recruited from a local rehabilitation center (Roessingh, Enschede) and two regional hospitals (Streekziekenhuis Koningin Beatrix, Winterswijk and Ziekenhuisgroep Twente, Almelo) in the Netherlands. Inclusion criteria for participants were: at least 6 months post-stroke; between 18 and 80 years old; movement limitations in the arm and/ or hand, but able to actively flex the elbow by at least $15^{\circ}$ and actively flex the finger(s) by at least $25 \%$ of the passive range of motion; living at home and having internet access; understand and follow instructions. Exclusion criteria were: orthopedic or neurological disease and/or pain restricting active range of motion of the upper extremity. All participants provided written informed consent. The study protocol was approved by the medical ethical committee Twente, Enschede, the Netherlands and is registered at the Netherlands Trial Registry (NTR3669). 


\section{Study design}

The study was a randomized controlled trial, in which participants trained independently at home for six weeks. Assignment to either the experimental or control group was performed at the end of the pre-training evaluation using concealed envelops, in blocks of two participants to ensure equal numbers of participants in each group. All participants were recommended to practice 30 minutes per day for six days per week, or beyond if they wished to, according to their preferred schedule. A trained clinical researcher visited each participant once per week to check on competence with the exercises, monitor progress and answer potential questions.

Participants in the experimental group consisted of the Dutch subgroup of the European SCRIPT study. ${ }^{8}{ }^{9}$ They used the SCRIPT dynamic wrist and hand orthosis, ${ }^{10}$ the SaeboMAS (Saebo Inc., Charlotte NC, USA) and a computer with touchscreen displaying gaming exercises (Figure 5.1). The SaeboMAS provided gravity compensation of the proximal arm. The instrumented wrist and hand orthosis is a custom-designed device providing extension forces to the wrist and fingers via passive leaf springs and elastic tension cords. ${ }^{10}$ The gaming exercises were controlled by arm and hand movements, recorded by sensors on the orthosis, and consisted of hand opening and closing, wrist flexion and extension, pronation and supination, and reaching forwards, backwards and sideways. The gaming exercises involved various difficulty levels to match individual performance levels. The researchers monitored progress and adjusted training programs remotely via a secured website. Further details of the experimental intervention are reported elsewhere. ${ }^{9}$

Participants in the control group performed conventional exercises using an existing home exercise program (Figure 5.1). ${ }^{11}$ The exercises involved three sets of increasing complexity to match the level of individual participants.
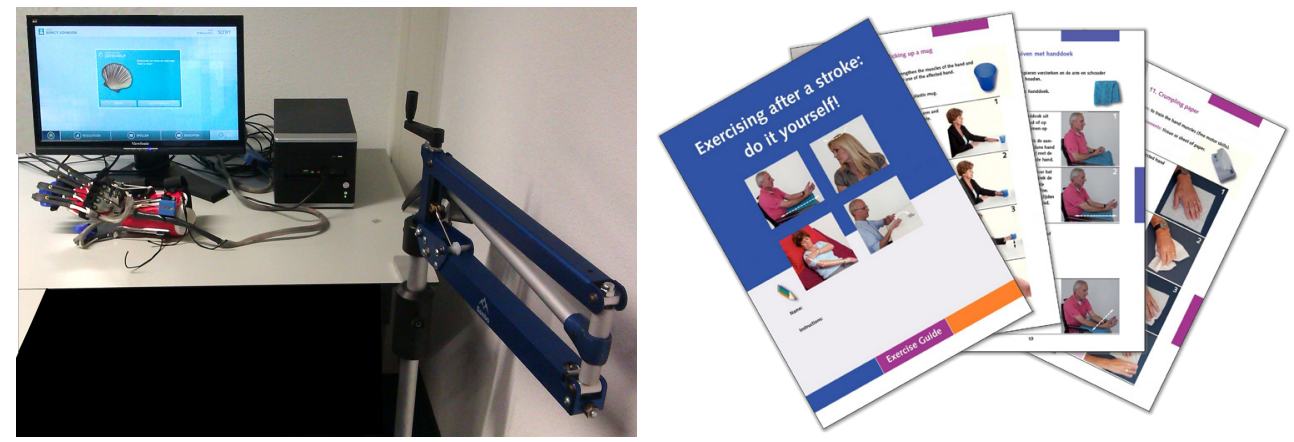

Figure 5.1 Training setup at home for the experimental group (left) and control group (right) Training for the experimental group involved the SCRIPT dynamic wrist and hand orthosis, ${ }^{10}$ the SaeboMAS (Saebo Inc., Charlotte NC, USA) and a computer with touchscreen displaying gaming exercises. Training for the control group involved conventional exercises from an exercise book. ${ }^{11}$ 


\section{Evaluation}

User acceptance was assessed in terms of training duration and motivation. Participants recorded self-reported frequency and duration of training in a diary. Daily training duration (in minutes) was summed over one week to provide the weekly training duration (in minutes). Weekly training duration averaged over six weeks represented average training duration (in minutes per week). Motivation was assessed post-training using the Intrinsic Motivation Inventory questionnaire. ${ }^{12,13} \mathrm{~A}$ higher score represents higher motivation, with a maximal score of seven and neutral score of four points.

Arm and hand function was evaluated at a research institute one week before training (preevaluation), within one week after training (post-evaluation) and two-months after the end of training (follow-up). Evaluation consisted of valid, standardized clinical assessments: FuglMeyer assessment and grip strength (body functions and structures level), Action Research Arm Test, Box and Block Test and Motor Activity Log (activity level) and Stroke Impact Scale (participation level). The assessor was not blinded to group assignment.

The upper extremity part of the Fugl-Meyer assessment determines the degree of synergydevelopment in the upper extremity (maximal score $=66$ ). ${ }^{14,15}$ Maximal hand grip strength was measured using a Jamar hand-held dynamometer, while the participant was seated with the upper arm adducted, elbow flexed $90^{\circ}$ and forearm in neutral position. The Action Research Arm Test evaluates coordination, dexterity and upper extremity function (maximal score $=57) \cdot{ }^{16,17}$ The Box and Block Test assesses manual dexterity in terms of number of blocks grasped, transported and released in one minute. ${ }^{18}$ The Motor Activity Log is a semistructured interview to assess perceived use of the paretic arm and hand (amount of use and quality of movement) during activities of daily life (maximal score $=5$ ). ${ }^{19}$ The Stroke Impact Scale evaluates quality of life by means of a self-reported questionnaire (maximal score $=100 \%) .^{20,21}$

\section{Statistical analysis}

Non-parametric methods were applied due to the small sample size. Statistical analyses were performed using IBM SPSS Statistics 19 for Windows with level of significance set at $\alpha<0.05$. In case of multiple comparisons, adjusted P-values in accordance with the HolmBonferroni correction were applied. ${ }^{22}$

Descriptive statistics (median with interquartile range) were used for all outcome measures. To examine differences between the experimental and control group, average training duration, Intrinsic Motivation Inventory scores and clinical assessment scores were compared using the Mann-Whitney test. Clinical assessment scores were analyzed across sessions using Friedman's Analysis of Variance. In case of significance, post hoc comparisons 
were done using the Wilcoxon signed ranks test. Additionally, correlation analyses were performed for training duration and changes in clinical assessments using Spearman's correlation coefficient.

\section{RESULTS}

Data is available from nine participants in the experimental and ten in the control group (Figure 5.2). At pre-evaluation, no differences were found between both groups, except for gender (Table 5.1).

There was a large amount of variation in training duration between individuals, ranging from 13 to 423 minutes per week. The weekly training duration remained stable during the training period (Figure 5.3). Averaged weekly training duration was lower $(P=0.025$, Table 5.2 ) for the experimental group (median = 118 minutes) than for the control group (median $=189$ minutes).

Motivation during training was positive in both groups, as reflected in a median score on the Intrinsic Motivation Inventory of 5.4 points for the experimental group and 4.9 points for the control group (Table 5.2). No difference was found between groups ( $P=0.935$ ).

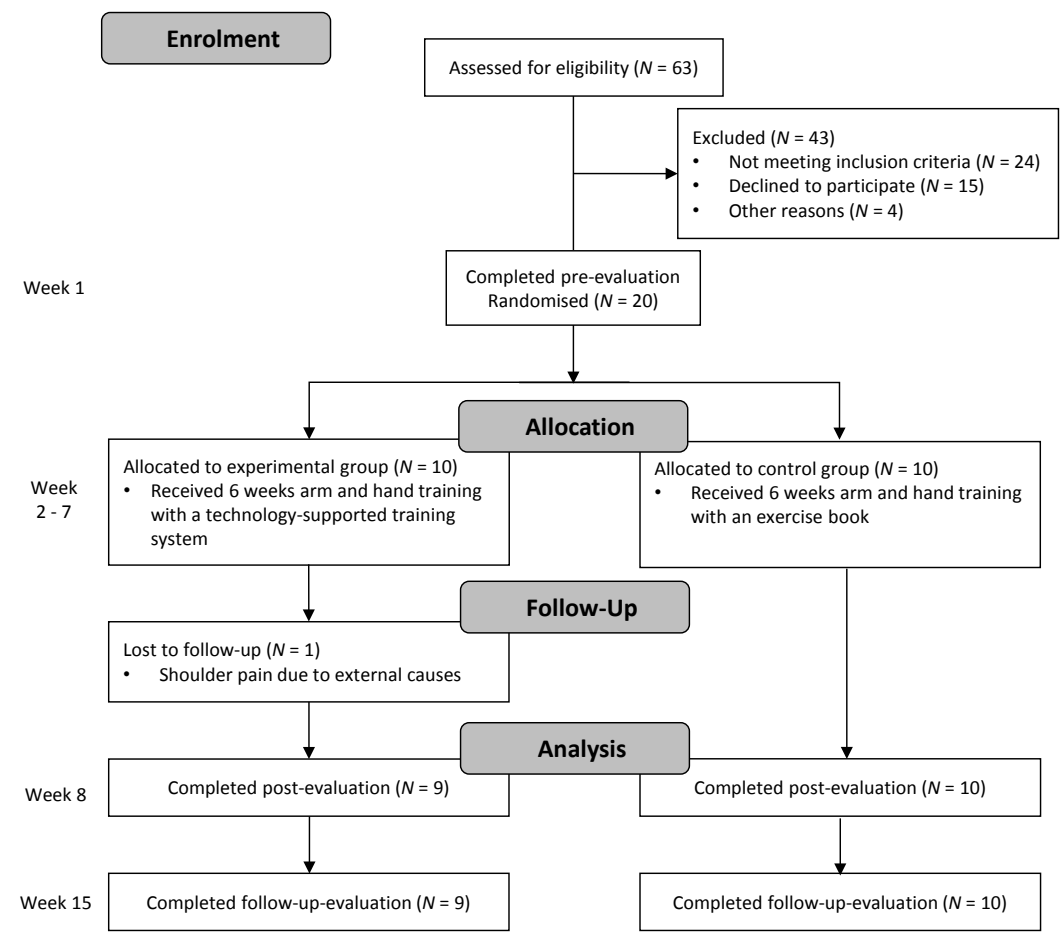

Figure 5.2 CONSORT flow diagram participant recruitment 
Table 5.1 Participant characteristics

\begin{tabular}{|c|c|c|}
\hline & Experimental group $(N=9)$ & Control group $(N=10)$ \\
\hline Gender (male/female) ${ }^{\mathrm{a}, \mathrm{c}}$ & $7 / 2$ & $3 / 7$ \\
\hline Age (years) ${ }^{b}$ & $58(48-65)$ & $62(54-70)$ \\
\hline Time post stroke (months) ${ }^{b}$ & $11(10-26)$ & $12(10-30)$ \\
\hline Type of stroke (infarction/hemorrhage) ${ }^{a}$ & $9 / 0$ & $7 / 3$ \\
\hline Affected body side (right/left) ${ }^{a}$ & $2 / 7$ & $6 / 4$ \\
\hline Dominant arm (right/left) ${ }^{\mathrm{a}}$ & $7 / 2$ & $7 / 3$ \\
\hline $\begin{array}{l}\text { Baseline Fugl-Meyer assessment score } \\
\text { (maximal } 66 \text { points) }^{\mathrm{b}}\end{array}$ & $44.0(11.5-51.0)$ & $35.0(20.3-48.0)$ \\
\hline $\begin{array}{l}\text { Baseline Action Research Arm Test score } \\
\text { (maximal } 57 \text { points) }^{\mathrm{b}}\end{array}$ & $31.0(3.5-50.0)$ & $25.0(3.8-30.8)$ \\
\hline Stroke severity (mild/moderate/severe) ${ }^{\mathrm{a}}$ & $2 / 3 / 4$ & $2 / 4 / 4$ \\
\hline
\end{tabular}

${ }^{a}$ Absolute numbers, ${ }^{b}$ median (interquartile range), ${ }^{c}$ statistically significant difference between groups

Self-reported training duration (experimental)

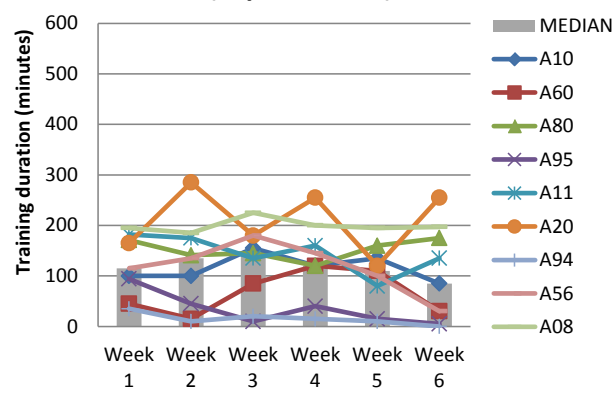

Self-reported training duration (control)

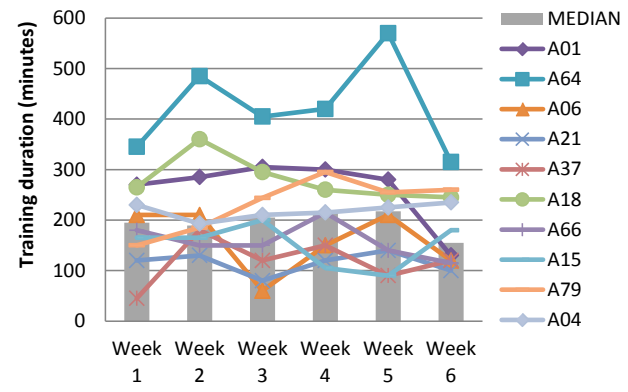

Figure 5.3 Individual (colored lines) and group (grey bars) results of self-reported training duration for the experimental group (left) and control group (right)

Both groups showed moderate improvements on most clinical assessments (Table 5.3). Multiple comparison analysis showed improvements between pre-evaluation and postevaluation for the Fugl-Meyer assessment $(P=0.011)$ and the Action Research Arm Test $(P=$ 0.016) for the control group, which were sustained at follow-up for the Action Research Arm Test $(P=0.011)$. Regarding between-group differences (Table 5.3), changes after training did not differ between groups for any of the outcome measures $(P \geq 0.165)$.

Training duration was strongly and positively correlated (Figure 5.4) with training-induced changes in the Box and Block Test $(\rho=0.69, P=0.001)$. Training duration showed a moderate but nonsignificant correlation with changes in Fugl-Meyer assessment ( $\rho=0.41, P=0.079$ ) and Action Research Arm Test ( $\rho=0.41, P=0.078)$. 
Table 5.2 Median (interquartile range) outcomes on user acceptance per group

\begin{tabular}{lccc}
\hline & Experimental group $(\boldsymbol{N}=9)$ & Control group $(\boldsymbol{N}=10)$ & P-Value \\
\hline $\begin{array}{l}\text { Average weekly training duration (minutes) } \\
\text { Intrinsic Motivation Inventory - } \\
\text { total scale (score) }\end{array}$ & $118(51-176)$ & 0.025 \\
$\begin{array}{l}\text { Intrinsic Motivation Inventory - } \\
\text { interest / enjoyment (score) }\end{array}$ & $5.4(3.4-6.7)$ & $4.9(4.7-6.8)$ & 0.935 \\
$\begin{array}{l}\text { Intrinsic Motivation Inventory - } \\
\text { perceived competence (score) }\end{array}$ & $4.9(3.7-6.3)$ & $5.4(2.6-7.0)$ \\
$\begin{array}{l}\text { Intrinsic Motivation Inventory - } \\
\text { effort / importance (score) }\end{array}$ & $4.7(1.8-6.2)$ & 0.838 \\
$\begin{array}{l}\text { Intrinsic Motivation Inventory - } \\
\text { perceived choice (score) }\end{array}$ & $5.0(3.8-7.0)$ & $0.074(4.4-7.0)$ \\
$\begin{array}{l}\text { Intrinsic Motivation Inventory - } \\
\text { pressure / tension (score) }\end{array}$ & $5.4(3.6-7.0)$ & 0.652 \\
$\begin{array}{l}\text { Intrinsic Motivation Inventory - } \\
\text { value / usefulness (score) }\end{array}$ & $5.6(4.0-7.0)$ & $5.0(2.8-7.0)$ & 0.902 \\
\hline
\end{tabular}
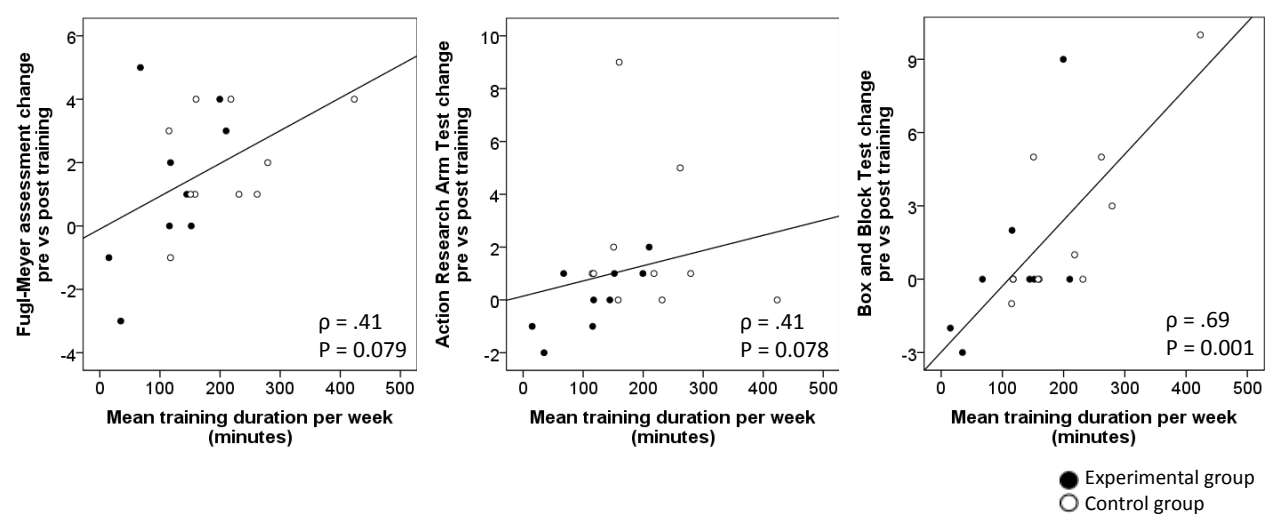

Figure 5.4 Correlations (indicated by $\rho$ with P-values) of averaged weekly training duration with training-induced changes in clinical outcome measures

\section{Discussion}

The present findings show that both technology-supported training and conventional exercises were accepted by stroke patients as self-administered treatment at home. Training was perceived as equally fun and motivating in both groups. Both groups reported a substantial amount of time spent on their exercises, with the control group reporting higher duration (189 versus 118 minutes per week). Changes in arm and hand function did not differ between groups. 


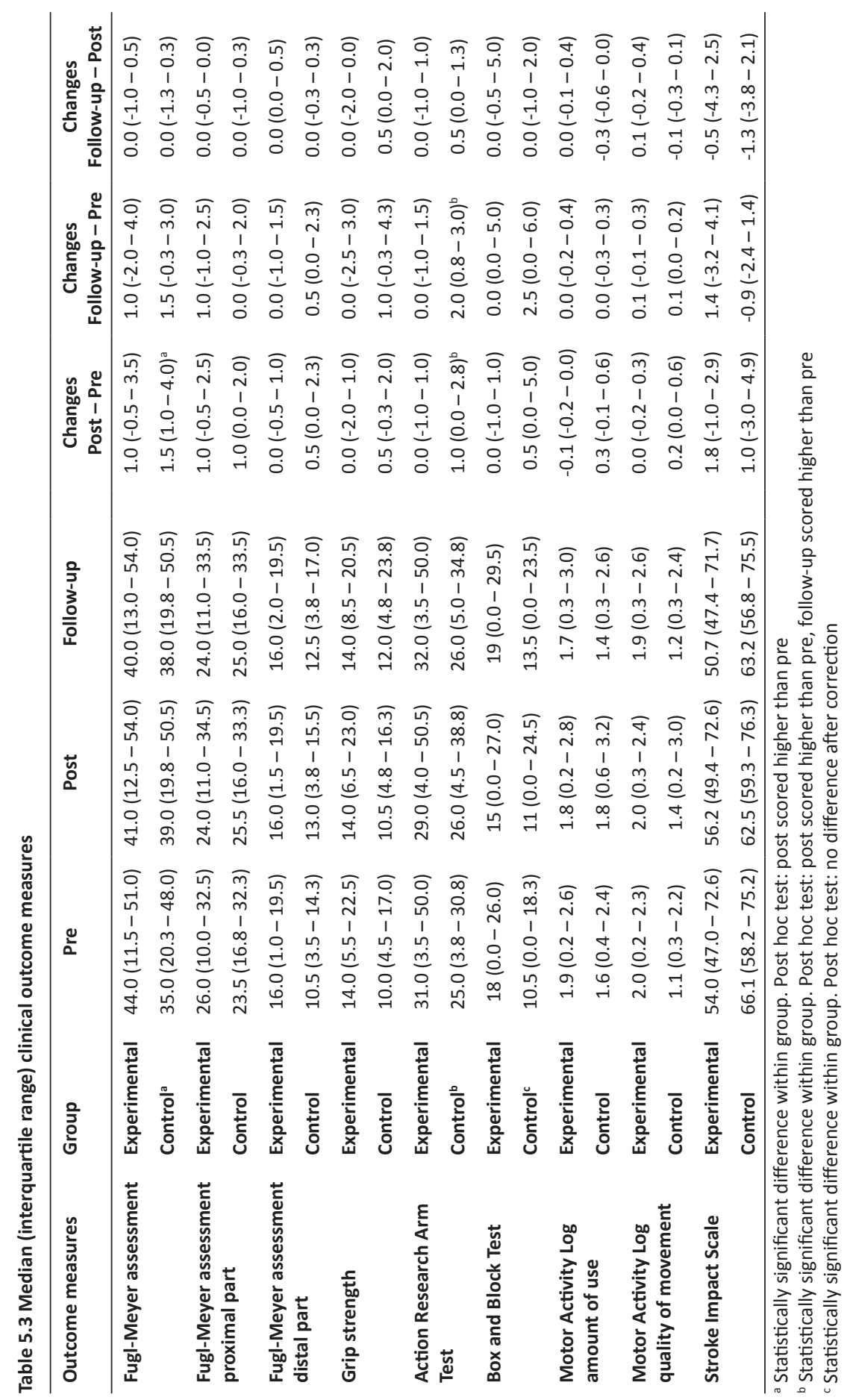


The current study is one of the first in which self-administered arm and hand training was evaluated in chronic stroke patients in their own home, without real-time therapist supervision. The acceptability of this type of technology-supported training at home is in line with another study investigating technology-supported treatment at home. ${ }^{23}$ Furthermore, it shows similar Intrinsic Motivation Inventory scores as interventions using rehabilitation technology in a clinical setting. ${ }^{24,25}$ The acceptability is also reflected in a fair amount of practice during first-ever use of the current system at home, suggesting that it is a promising way to enable independent practice.

The lack of differences in effect on arm and hand function between technology-supported and conventional therapy is comparable with studies performed in a clinical setting ${ }^{1,5}$ and at home. ${ }^{26}$ The extent of arm and hand function improvements is in line with other studies into home-based rehabilitation in chronic stroke. ${ }^{23,27}$ Wolf et al. ${ }^{26}$ did find clinically relevant improvements after home-based telemonitored robotic-assisted therapy in patients less than six months post-stroke. ${ }^{26}$ This indicates that home-based training can be considered at an early stage after stroke, to take optimal advantage of the time window for recovery. ${ }^{28}$

The limited improvements after technology-supported training may be related to the inability to achieve the recommended training duration of 180 minutes per week. A minimal dose of 16 hours of additional training is suggested to increase the chance of achieving clinically relevant treatment effects. ${ }^{29}$ The actual training duration in the present study amounted to 11.8 hours. This has likely been insufficient to generate consistent improvements in all patients, which is in line with some other studies investigating technology-supported arm and hand training at home. ${ }^{23,27}$ The importance of training duration, regardless of training content, is emphasized by the positive correlation between training duration and traininginduced changes in arm and hand function.

To enhance training duration or adherence, more attention towards motivational strategies is needed when applying technology for self-administered practice. For example, in the present study three games were available for the experimental group, versus maximally 34 exercises for the control group. This may have played a role in the higher training duration of the control group. A large variety of attractive functional exercises is important to prevent boredom and abandonment. ${ }^{6}$ In addition, regular contact between patients and a professional has a motivational effect and increases therapy adherence. ${ }^{30,31}$ Indeed, the weekly home visits were highly appreciated by the participants in both groups.

The present findings should be interpreted with care, due to several limitations of the present study. Firstly, neither the assessor nor the participants were blinded to group assignment. Secondly, the current study represents a first, explorative insight into the acceptability of 
self-administered technology-supported training at home based on a limited number of participants. The small sample size limits the generalizability of the results. Thirdly, there was a difference in gender distribution between groups. However, patient adherence to conventional physical therapy was reported to be not different between men and women. ${ }^{32}$ In the present study, results were not remarkably different between men and women either. Lastly, a possible inaccurate recall and overestimation of the home-based exercises may have caused a discrepancy in the self-reported training duration between both groups. ${ }^{33}$ The experimental group knew that their training duration was recorded objectively within the training system as well, likely leaving less room for inaccurate recall or overestimation compared to the control group.

An additional benefit of technology-supported training over conventional arm and hand exercises at home was not demonstrated in the present study. At this point the available research suggests that training duration in itself is a major contributor for changes in arm and hand function, regardless of the training content. On the other hand, technologysupported training at home was accepted by stroke patients. Several issues, especially with respect to improving adherence, should be investigated further. This may allow optimization of technology to support treatment at home, and would require re-evaluation before a conclusion can be drawn about its added benefit. Based on results of the current study, a power calculation shows that a future randomized controlled trial would require at least 25 participants per group.

\section{Clinical message}

- Technology-supported treatment at home was accepted, but was associated with a lower level of practice than conventional treatment.

- The additional benefit of technology-supported training over conventional exercises at home was not demonstrated.

- Training duration in itself is a major contributor for changes in arm and hand function

\section{REFERENCES}

1. Maciejasz P, Eschweiler J, Gerlach-Hahn K, Jansen-Troy A and Leonhardt S. A survey on robotic devices for upper limb rehabilitation. Journal of Neuroengineering and Rehabilitation. 2014; 11: 3.

2. Prange GB, Jannink MJ, Groothuis-Oudshoorn CG, Hermens HJ and ljzerman MJ. Systematic review of the effect of robot-aided therapy on recovery of the hemiparetic arm after stroke. J Rehabil Res Dev. 2006; 43: 171-84.

3. Mehrholz J, Pohl M, Platz T, Kugler J and Elsner B. Electromechanical and robot-assisted arm training for improving activities of daily living, arm function, and arm muscle strength after stroke. Cochrane Database Syst Rev. 2015; 11: CD006876.

4. Basteris A, Nijenhuis SM, Stienen AH, Buurke JH, Prange GB and Amirabdollahian F. 
Training modalities in robot-mediated upper limb rehabilitation in stroke: a framework for classification based on a systematic review. J Neuroeng Rehabil. 2014; 11: 111.

5. Pollock A, Farmer SE, Brady MC, et al. Interventions for improving upper limb function after stroke. The Cochrane database of systematic reviews. 2014; 11: CD010820.

6. Timmermans AA, Seelen HA, Willmann RD and Kingma H. Technology-assisted training of arm-hand skills in stroke: concepts on reacquisition of motor control and therapist guidelines for rehabilitation technology design. J Neuroeng Rehabil. 2009; 6: 1.

7. Balasubramanian S, Klein J and Burdet E. Robot-assisted rehabilitation of hand function. Curr Opin Neurol. 2010; 23: 661-70.

8. Amirabdollahian F, Ates S, Basteris A, et al. Design, development and deployment of a hand/wrist exoskeleton for home-based rehabilitation after stroke - SCRIPT project. Robotica. 2014; 32: 1331-46.

9. Nijenhuis SM, Prange GB, Amirabdollahian F, et al. Feasibility study into self-administered training at home using an arm and hand device with motivational gaming environment in chronic stroke. Journal of Neuroengineering and Rehabilitation. 2015; 12: 89.

10. Ates S, Lobo-Prat J, Lammertse P, van der Kooij H and Stienen AH. SCRIPT Passive Orthosis: Design and technical evaluation of the wrist and hand orthosis for rehabilitation training at home. IEEE International Conference on Rehabilitation Robotics. 2013; 2013: 1-6.

11. Nederlandse CVA-vereniging Samen Verder, Kenniscentrum Revalidatiegeneeskunde Utrecht and Werkgroep CVA Nederland. Oefengids: Zelf oefenen na een beroerte (CVA). 4e herziene druk ed. Utrecht 2012. [Dutch Stroke Society, Center of Excellence in Rehabilitation Medicine Utrecht, Dutch Stroke Working Group. Exercise guide: exercising after a stroke; do it yourself! Utrecht 2012; 4th rev ed.] Available at: http://www.cvavereniging.nl/PDF2013/Oefengids4edruk.pdf, www.snelinbeweging.nl (accessed 22 December 2015).

12. Ryan RM. Control and information in the intrapersonal sphere: An extension of cognitive evaluation theory. Journal of Personality and Social Psychology. 1982; 43: 450-61.

13. McAuley E, Duncan $T$ and Tammen VV. Psychometric properties of the Intrinsic Motivation Inventory in a competitive sport setting: a confirmatory factor analysis. Res Q Exerc Sport. 1989; 60: 48-58.

14. Fugl-Meyer AR, Jaasko L, Leyman I, Olsson S and Steglind S. The post-stroke hemiplegic patient. 1. A method for evaluation of physical performance. Scand J Rehabil Med. 1975; 7: 13-31.

15. Deakin A, Hill H and Pomeroy VM. Rough Guide to the Fugl-Meyer Assessment: Upper limb section. Physiotherapy. 2003; 89: 751-63.

16. Lyle RC. A performance test for assessment of upper limb function in physical rehabilitation treatment and research. Int J Rehabil Res. 1981; 4: 483-92.

17. Yozbatiran N, Der-Yeghiaian L and Cramer SC. A standardized approach to performing the action research arm test. Neurorehabil Neural Repair. 2008; 22: 78-90.

18. Mathiowetz V, Volland G, Kashman N and Weber K. Adult norms for the Box and Block Test of manual dexterity. Am J Occup Ther. 1985; 39: 386-91.

19. van der Lee JH, Beckerman $\mathrm{H}$, Knol DL, de Vet $\mathrm{HC}$ and Bouter LM. Clinimetric properties of the motor activity log for the assessment of arm use in hemiparetic patients. Stroke. 2004; 35: 1410-4.

20. van de Port IGL, Leendes K, Sellmeijer D, Zuidgeest A and Kwakkel G. Betrouwbaarheids en concurrente validiteit van de Nederlandse Stroke Impact Scale 2.0 bij patienten met een CVA. Ned Tijdschrift Fysiotherapie. 2008; 118: 12-8.

21. Duncan PW, Wallace D, Lai SM, Johnson D, Embretson S and Laster LJ. The stroke impact scale version 2.0. Evaluation of reliability, validity, and sensitivity to change. Stroke. 1999; 30: 2131-40. 
22. Holm S. A simple sequentially rejective multiple test procedure. Scandinavian journal of statistics. 1979; 6: 65-70.

23. Sivan M, Gallagher J, Makower S, et al. Home-based Computer Assisted Arm Rehabilitation (hCAAR) robotic device for upper limb exercise after stroke: results of a feasibility study in home setting. Journal of Neuroengineering and Rehabilitation. 2014; 11: 163.

24. Prange GB, Kottink Al, Buurke JH, et al. The Effect of Arm Support Combined With Rehabilitation Games on Upper-Extremity Function in Subacute Stroke: A Randomized Controlled Trial. Neurorehabil Neural Repair. 2015; 29: 174-182.

25. Colombo R, Pisano F, Mazzone A, et al. Design strategies to improve patient motivation during robot-aided rehabilitation. J Neuroeng Rehabil. 2007; 4: 3.

26. Wolf SL, Sahu K, Bay RC, et al. The HAAPI (Home Arm Assistance Progression Initiative) Trial: A Novel Robotics Delivery Approach in Stroke Rehabilitation. Neurorehabilitation and Neural Repair. 2015; 29: 958-68.

27. Donoso Brown EV, McCoy SW, Fechko AS, Price R, Gilbertson T and Moritz CT. Preliminary investigation of an electromyography-controlled video game as a home program for persons in the chronic phase of stroke recovery. Arch Phys Med Rehabil. 2014; 95: 14619.

28. Kwakkel G, Kollen BJ and Wagenaar RC. Long term effects of intensity of upper and lower limb training after stroke: a randomised trial. J Neurol Neurosurg Psychiatry. 2002; 72: 473-9.

29. Kwakkel G, van Peppen R, Wagenaar RC, et al. Effects of augmented exercise therapy time after stroke: a meta-analysis. Stroke. 2004; 35: 2529-39.

30. Jette $A M$, Rooks $D$, Lachman $M$, et al. Home-based resistance training: predictors of participation and adherence. Gerontologist. 1998; 38: 412-21.

31. Williams AB, Fennie KP, Bova CA, Burgess JD, Danvers KA and Dieckhaus KD. Home visits to improve adherence to highly active antiretroviral therapy: a randomized controlled trial. J Acquir Immune Defic Syndr. 2006; 42: 314-21.

32. Sluijs EM, Kok GJ and van der Zee J. Correlates of exercise compliance in physical therapy. Physical therapy. 1993; 73: 771-82; discussion 83-6.

33. Myers $L$ and Midence $K$. Concepts and issues in adherence. In: Myers $L$ and Midence $\mathrm{K},($ eds.). Adherence to Treatment in Medical Conditions. Amsterdam: Taylor \& Francis, 1998, p. 1-24. 



\section{Chapter 6}

Strong relations of elbow excursion and grip strength with post stroke arm function and activities: should we aim for this in technology-supported training?

Nijenhuis SM, Prange-Lasonder GB, Fleuren JFM, Wagenaar J, Buurke JH, Rietman JS Submitted to Journal of Rehabilitation and Assistive Technologies Engineering 


\section{ABSTRACT}

Objective: To investigate the relationships between an extensive set of objective movement execution kinematics of the upper extremity and clinical outcome measures in chronic stroke patients: at baseline and after technology-supported training at home.

Methods: Twenty mildly to severely affected chronic stroke patients participated in the baseline evaluation, 15 were re-evaluated after six weeks of intensive technology-supported or conventional arm/hand training at home. Grip strength, 3D motion analysis of a reachand-grasp task and clinical scales (Fugl-Meyer assessment (FM), Action Research Arm Test (ARAT) and Motor Activity Log (MAL)) were assessed pre and post training.

Results: Most movement execution parameters showed moderate-to-strong relationships with FM and ARAT, and to a smaller degree with MAL. Elbow excursion explained the largest amount of variance in FM and ARAT, together with grip strength. The only strong association after training was found between changes in ARAT and improvements in hand opening (conventional) or grip strength (technology-supported).

Conclusions: Elbow excursion and grip strength showed strongest association with poststroke arm function and activities. Improved functional ability after training at home was associated with increased hand function. Addressing both reaching and hand function are indicated as valuable targets for (technological) treatment applications to stimulate functional improvements after stroke. 


\section{INTRODUCTION}

Upper extremity hemiparesis is a major problem in patients with stroke, affecting their independence in performance of daily life activities. ${ }^{1}$ Therefore, optimal recovery of arm and hand function is an important goal in stroke rehabilitation. Essential treatment aspects for neurorehabilitation are intensive practice with active engagement of the patient, performing meaningful task-specific exercises in a high dose. ${ }^{2-4}$ Technology-supported treatment can facilitate independent, self-administered training with many repetitions and enhance the dosage of treatment, especially when applied in a (partly) therapist-independent setting, for instance at home. Although several studies have shown that technology-supported interventions are effective to improve upper extremity motor function after stroke, their influence on activity level is less understood. ${ }^{5-7}$ This might be explained by the fact that many of those technology-supported interventions are focused on body function level, even though ultimately its impact is desired on activity level. ${ }^{6}$

Another factor which might contribute to the limited understanding of how technologysupported interventions can influence performance on activity level is an inappropriate choice of outcome measures. ${ }^{8-10}$ Inclusion of outcome measures covering all domains of the International Classification of Functioning, Disability and Health (ICF) is recommended. ${ }^{11}$ However, when clinical outcome measures are applied to quantify those domains, improvements on for instance activity level cannot be attributed to either recovery or compensation. 'Recovery' is used in this paper to describe improvements resulting from restitution or repair of structures and functions, 'compensation' is defined as the appearance of alternative movement patterns or the use of alternate joints or end effectors during the accomplishment of a task. ${ }^{12}$ In order to differentiate between recovery and compensation, more detailed information of movement patterns and strategies is needed, collected in an objective and reliable way. ${ }^{12-14}$ This is usually not part of standardized clinical outcome measures since they mainly focus on task accomplishment. However, kinematic movement analysis can provide valuable information on the quality of functional task performance, at least when assessed in a research setting.

With a better understanding of the relation between objective movement execution parameters of the affected arm and hand after stroke (as assessed via kinematics and grip strength) and sensorimotor function or activity limitations (as assessed via clinical outcome measures), we gain more insight into underlying mechanisms and may be able to specify areas of attention for (design of) upper extremity interventions. ${ }^{12}{ }^{15}, 16$ Moreover, the effect of an intervention on restoration of function by recovery or compensation might be distinguished, in order to better understand how the intervention affects the restoration capacity of a patient. 
Previous research in stroke patients showed significant relations between kinematic outcomes measured during a reach and grasp task and sensorimotor function and activity limitation of the upper extremity. ${ }^{17,18}$ Movement smoothness and total movement time, together with compensatory trunk displacement were associated with activity capacity (assessed by the Action Research Arm Test (ARAT)). ${ }^{17}$ Another study found trunk displacement alone explaining the majority of variance in sensorimotor function (assessed by the Fugl-Meyer assessment (FM)). ${ }^{18}$ Kinematic variables assessed during a reaching task (including endpoint variables, trunk involvement, joint recruitment and interjoint coordination) were significant predictors for improvement in self-perceived activity performance in daily life, as measured with the Motor Activity Log (MAL) in mild to moderate chronic stroke. ${ }^{19}$ These studies show partly overlapping results on one hand, but on the other hand tend to differ, with various kinematic outcome measures used in different tasks. A study with a comprehensive set of movement execution parameters is desired in order to provide a more complete picture.

Therefore, we determined the relationships between an extensive set of movement execution parameters (measured via kinematics) during a functional reach and grasp task and grip strength, and outcomes on sensorimotor function, activity capacity and selfperceived activity performance (measured via clinical outcome measures) in mildly to severely affected chronic stroke patients. To obtain a more in-depth insight into the role of recovery versus compensation, we examined whether and how training-induced changes in movement execution parameters were related to training-induced changes in clinical outcome measures after technology-supported or conventional arm and hand training at home.

\section{Methods}

\section{Participants}

Kinematic data obtained during two previous studies on chronic stroke patients within the Supervised Care and Rehabilitation Involving Personal Telerobotics (SCRIPT) project ${ }^{20}$ were combined in the current work for additional analysis: a cross-sectional measurement in which direct effects of a passive dynamic wrist and hand orthosis on hand and arm movement kinematics were assessed ${ }^{21}$ and a randomized controlled trial (RCT) with six weeks of intensive, self-administered arm and hand training at home. ${ }^{22}$ All participants signed informed consent forms before inclusion into either study, approved by the medical ethical committee Twente, Enschede, the Netherlands and registered at the Netherlands Trial Registry (NTR3669). Both studies had the same inclusion criteria: $>6$ months post stroke, age between 18 and 80, movement limitations in the arm and/or hand, but with at least 15 degrees active elbow flexion and able to actively flex the finger(s) by at least $25 \%$ of the passive range of motion, live at home with internet access, and able to understand and follow instructions. Exclusion criteria were: orthopedic or neurological disease and/or pain 
restricting active range of motion of the upper extremity.

\section{Procedures}

During a cross-sectional measurement (PRE), 20 participants underwent clinical measurements to evaluate status of arm and hand function and dexterity, hand grip dynamometry to measure maximal grip strength and a functional reach and grasp task to determine movement execution. A subset of 15 participants repeated the same measurements after six weeks of intensive training for the arm and hand at home (POST). These participants used either a technology-supported training system (experimental group) or performed conventional exercises from an exercise book (control group). The details of both interventions are described elsewhere. ${ }^{22}$

\section{Clinical outcome measures}

The sensorimotor function of the arm was measured with the upper extremity part of the Fugl-Meyer assessment (FM). The FM assesses the ability to perform isolated movements of the arm, wrist, hand and coordination within and out of synergy. The maximal score is 66. ${ }^{23,24}$ Activity capacity was evaluated by the Action Research Arm Test (ARAT). The ARAT evaluates dexterity on the subtests grasp, grip, pinch and gross arm movements, with a maximal score of 57. ${ }^{25,26}$ The Motor Activity Log (MAL) was used to assess self-perceived activity performance, in terms of amount of use (AOU) and quality of movement (QOM) of the paretic arm and hand during activities of daily life. The MAL is a semi-structured interview with 26 items and has a maximal score of five for both subsections. ${ }^{27}$

\section{Grip strength}

The maximal grip strength of the affected hand was measured using a hand-held dynamometer, while the participants sat on a chair, with the shoulder adducted, the elbow flexed 90 degrees and neutral position of the forearm and wrist. Participants were verbally encouraged to squeeze the dynamometer with maximal strength. The best result from three repetitions, separated by 15 seconds of rest, was used for analysis. ${ }^{28}$

\section{Reach and grasp task}

A standardized reach and grasp task was performed to measure upper extremity movement execution during a functional task, related to activities of daily living. Each participant sat on a chair, with the shoulder adducted, the elbow in 90 degrees flexion, with the palm of the hand resting on the table in front of the participant (Point $A$ in Figure 6.1). The reach and grasp task involved four phases: forward reaching to a bottle with diameter of six $\mathrm{cm}$ placed on the table and grasping it (Point B in Figure 6.1), holding the bottle while moving the arm to the start position (Point A), bringing it back to the original position (point B) on the table and releasing the bottle, and returning the hand to the start position. The distance of the 
start position to the bottle was determined by near-maximal (approximately $80 \%$ ) active forward reach at the start of the task. The participant was instructed to perform the reach and grasp task with the affected arm and hand, at a comfortable, self-selected speed for about ten repetitions. The trunk was not constrained during the reach and grasp task and compensatory trunk movements were allowed and measured.

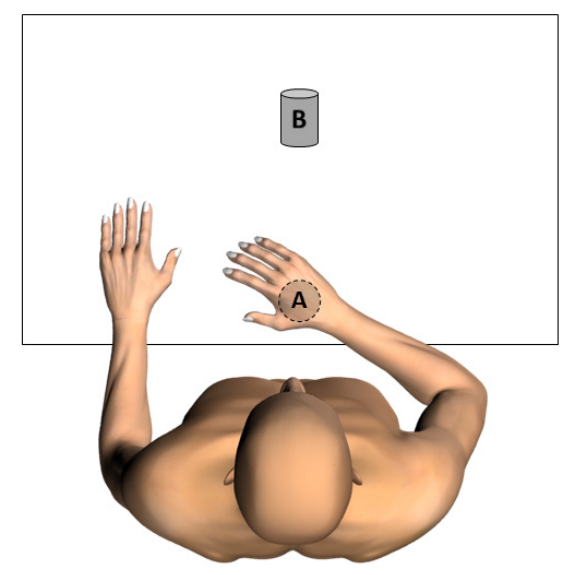

Figure 6.1 Measurement setup during reach and grasp task

\section{Kinematic data analysis}

Upper extremity movement kinematics were recorded during the reach and grasp task, using a 3D motion analysis system (VICON MX13+ motion capture system, Oxford Metrics, Oxford, UK). Six infrared cameras captured movements of the arm and hand by recording of reflective markers. These markers were placed on predefined points of the thorax and upper extremity according to the guidelines of the International Society of Biomechanics for the $\mathrm{arm}^{29}$ and an adapted version of a validated marker model for the hand (Figure 6.3). ${ }^{30}$

The captured VICON data were analyzed using VICON Nexus 1.8.2 and transferred to MATLAB (R2013b, MathWorks Inc., Natick, Massachusetts, USA,) for custom, offline analysis. The data were filtered with a second order low pass Butterworth filter of $20 \mathrm{~Hz}$ and zero phase shift. For each participant, the average of the seven repetitions with largest hand opening was used for further analysis. For participants with fewer useful repetitions available, for example in case of a poor data sample, the average of at least three useful repetitions was used. For participants without useful data on hand opening, for example in case of problems grasping the bottle, the average of the seven middle trials was used in statistical analysis. No data on hand opening are available for these participants in the results. Data were recorded from all four phases in the reach and grasp task, but only data from the first phase (the reach to grasp phase) were used for analysis in this paper (Figure 6.2). 


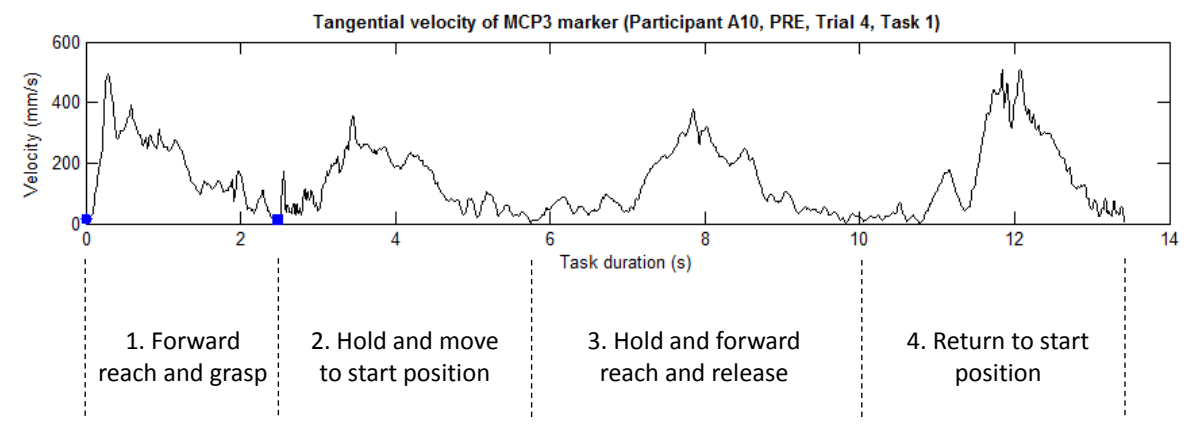

Figure 6.2 Four phases of the reach and grasp task

Shown is the velocity profile $(\mathrm{mm} / \mathrm{s})$ of the hand marker. The dot and square represent the start and end of the reach to grasp phase, respectively.

The following kinematic variables were calculated from the 3D position data during this first reach to grasp phase. The maximal velocity $(\mathrm{mm} / \mathrm{s})$ was defined as the maximal of the tangential velocity profile of the hand marker (MCP3 in Figure 6.3). The movement time (MT) was defined as the time (s) participants needed to perform the first phase of the reach and grasp task. Movement onset and offset were defined as the moment at which the tangential velocity of the hand marker exceeded and dropped below $2 \%$ of the maximal velocity, respectively. ${ }^{31}$ Movement smoothness (MS) was defined as the number of movement units (nmu) in the tangential velocity profile of the hand marker, which was searched for local minima and maxima. According to Alt Murphy et al. 2011, a difference between a minimum and next maximum value exceeding the amplitude limit of $20 \mathrm{~mm} / \mathrm{s}$ indicated a velocity peak, if the time between two subsequent peaks was at least 150 milliseconds. ${ }^{31}$ The maximal hand opening $(\mathrm{HO})$ was determined as the maximal Euclidean distance $(\mathrm{mm})$ between the tip of the thumb and the tip of the middle finger. Although the transport and grasp components predominantly occur simultaneously (in parallel) in most healthy people, stroke patients often prefer serial processing of movements. ${ }^{32}$ The moment of maximal hand opening relative to the moment of maximal hand speed was used as a measure of temporal pattern, using the formula: ${ }^{14}$

$$
\text { Temporal pattern }=\frac{\text { Moment maximal hand opening }}{\text { Moment maximal velocity }} * 100 \%
$$

Forward trunk displacement (TD) was defined as the difference between the maximal and minimal forward displacement $(\mathrm{mm})$ of the trunk marker (Th2) in the sagittal plane. Thoracohumeral joint angles were calculated according to the recommendations of the International Society of Biomechanics. ${ }^{29}$ Joint excursions of the elbow, wrist and shoulder were calculated as the difference between maximal and minimal joint angles (degrees) during the first phase of the reach and grasp task. Elbow flexion and extension excursion 
(EE) was defined as the joint angle between the forearm and the humerus. Wrist flexion and extension excursion (WE) was calculated by the angle between the vectors joining the wrist and forearm markers and wrist and hand markers. The shoulder joint orientation (Figure 6.3) was represented by the elevation plane (EP), elevation angle (EA) and axial rotation (AR). The EP was defined as the angle between the humerus and a virtual line through the shoulders, viewed in the transversal plane. The EA represented the angle between the humerus and thorax, in the plane of elevation. The AR was defined as the rotation around a virtual line from the glenohumeral joint to the elbow joint.

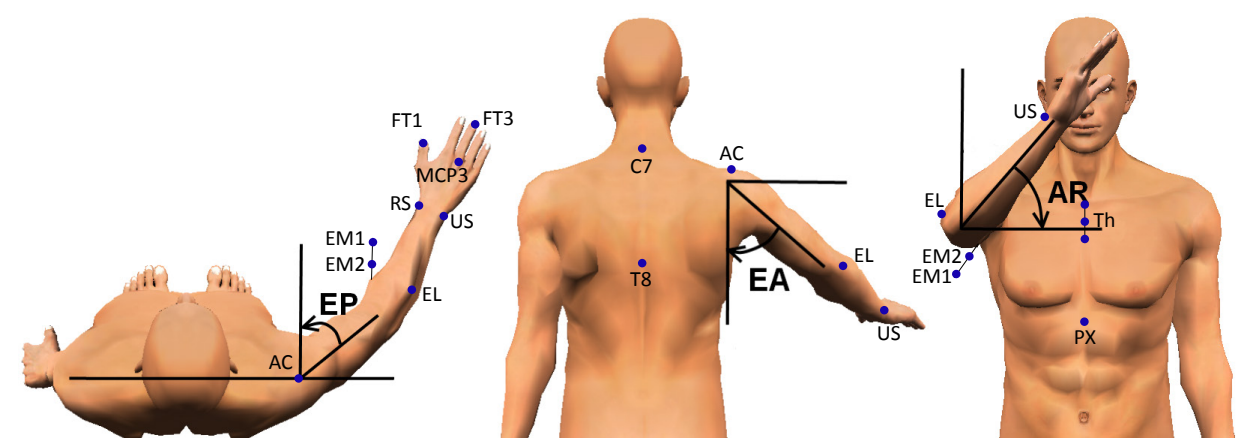

Figure 6.3 Joint angles of the shoulder and marker positions (adapted from Krabben et al. 2012 ${ }^{33}$ ) Abbreviations: EP: Elevation Plane, EA: Elevation Angle, AR: Axial Rotation, PX: processus xiphoideus, C7: 7th cervical vertebra, T8: 8th thoracal vertebra, Th: thorax markers on a triangular frame with Th1: upper marker on incisura jugularis, Th2: middle marker on sternum, Th3: lower marker on sternum, AC: acromioclavicular joint, EL: lateral epicondyle, EM2: medial epicondyle (proximal marker on pointer), EM1: medial epicondyle (distal marker on pointer), US: ulnar styloid, RS: radial styloid, MCP3: metacarpophalangeal 3, FT1: distal phalanx of the thumb, FT3: distal phalanx of the third finger

\section{Statistical analyses}

Statistical analyses were performed using IBM SPSS Statistics 22 for Windows with level of significance set at $\alpha<0.05$. All outcome measures were inspected for normal distribution using histogram plots including normal curves and normal probability plots, and ShapiroWilk tests, prior to selection of appropriate statistical tests. Descriptive statistics (mean with standard deviation (SD)) were used for all outcome measures.

Relationships between clinical outcome measures (FM, ARAT and MAL), grip strength and kinematic variables at PRE were evaluated using Pearson's or Spearman's correlation coefficient. To examine which predictor or combination of predictors explained the greatest amount of variance in clinical outcome measures, multiple linear regression with forward deletion was used. Only kinematic variables which showed strong significant correlations ( $r$ $\geq .70$ ) with the clinical assessments were entered into the regression model, in addition to known kinematic variables which show correlations with clinical outcome measures after stroke. ${ }^{17,18}$ Probability for entry in forward regression was set at .05 and removal at .10 . 
Prior to these analyses, tests were done to ensure no violation of assumptions of normality, linearity and homoscedasticity by checking histogram plots including normal curves and normal probability plots of the residuals and the scatterplot of standardized residuals against standardized predicted values. Multicollinearity among the predictors was checked by inspecting the individual correlations among predictors and tested by the criterion of a variance inflation factor greater than 10 . In cases of very strong correlations $(\geq 0.80)$ between predictors, one predictor is substituted for another.

Considering training-induced changes, outcomes on the clinical measures, grip strength and kinematic variables were analyzed between sessions (PRE versus POST) using the dependent t-test or Wilcoxon signed ranks test. To examine differences between the experimental and control group, clinical outcome scores, grip strength and kinematic variables were compared using the independent t-test or Mann-Whitney test, at PRE, POST and mean change.

In addition, correlation analyses were performed for training-induced changes on clinical outcome measures (FM, ARAT and MAL) and changes in grip strength and kinematic variables using Pearson's or Spearman's correlation coefficient.

\section{RESULTS}

\section{Participants}

Cross-sectional baseline data from 20 participants were analyzed. From 15 of those participants post training data was available as well, divided randomly between a control group ( $N=8$ ) and experimental group ( $N=7$ ). Participants showed a large variation of stroke severity, ranging from severely to mildly impaired patients, based on baseline FM score. ${ }^{34}$ Considering participant characteristics at baseline (Table 6.1), there were no differences between the two training groups.

\section{Cross-sectional measurement PRE training}

\section{Correlations}

Sensorimotor function (FM) correlated strongly $(r \geq .70)$ with grip strength, maximal velocity and elbow excursion. FM correlated moderately $(r \geq .40)$ with movement time (negatively), maximal hand opening, trunk displacement (negatively), and shoulder excursion axial rotation (Table 6.2). The same kinematic variables correlated with activity capacity (ARAT), with comparably strong associations (Table 6.2). Self-perceived activity performance (MAL) correlated with grip strength, maximal hand opening, elbow excursion and shoulder excursion axial rotation, but the associations were slightly weaker for elbow excursion (Table 6.2). An example of a relation between a clinical outcome measure (FM) and kinematic variable (elbow excursion) is displayed in Figure 6.4. 
Table 6.1 Participant characteristics at baseline (absolute numbers or mean (standard deviation))

\begin{tabular}{|c|c|c|c|}
\hline & \multirow{2}{*}{$\begin{array}{c}\text { Cross-sectional } \\
\text { measurement PRE } \\
\text { All participants } N=20\end{array}$} & \multicolumn{2}{|c|}{ Training data PRE $(N=15)$} \\
\hline & & Control $N=8$ & Experimental $N=7$ \\
\hline Gender & 12 male / 8 female & 3 male / 5 female & 6 male / 1 female \\
\hline Age in years & $60(11)$ & $61(10)$ & $57(12)$ \\
\hline Months post stroke & $21(18)$ & $27(26)$ & $16(10)$ \\
\hline Type of stroke & $\begin{array}{c}16 \text { infarction / } \\
3 \text { hemorrhage /1 unknown }\end{array}$ & $\begin{array}{l}6 \text { infarction / } \\
2 \text { hemorrhage }\end{array}$ & $\begin{array}{l}7 \text { infarction / } \\
0 \text { hemorrhage }\end{array}$ \\
\hline Affected body side & 12 left / 8 right & 4 left / 4 right & 5 left / 2 right \\
\hline Dominant arm & 3 left / 17 right & 2 left / 6 right & 1 left / 6 right \\
\hline $\begin{array}{l}\text { Fugl-Meyer score } \\
\text { (maximal } 66 \text { points) }\end{array}$ & $40(15)$ & $38(12)$ & $40(18)$ \\
\hline $\begin{array}{l}\text { Action Research Arm Test score } \\
\text { (maximal } 57 \text { points) }\end{array}$ & $28(18)$ & $26(16)$ & $33(21)$ \\
\hline Stroke severity & $\begin{array}{c}7 \text { mild / } 8 \text { moderate / } \\
5 \text { severe }\end{array}$ & $\begin{array}{c}2 \text { mild / } 4 \text { moderate / } \\
2 \text { severe }\end{array}$ & $\begin{array}{c}2 \text { mild / } 3 \text { moderate / } \\
2 \text { severe }\end{array}$ \\
\hline
\end{tabular}

Table 6.2 Correlation coefficients between clinical outcome measures and kinematic variables

\begin{tabular}{lcccc}
\hline & $\begin{array}{c}\text { FM score } \\
(\mathrm{max} 66)^{\mathrm{a}}\end{array}$ & $\begin{array}{c}\text { ARAT score } \\
(\mathrm{max} 57)\end{array}$ & $\begin{array}{c}\text { MAL AOU } \\
(0-5)\end{array}$ & $\begin{array}{c}\text { MAL QOM } \\
(0-5)\end{array}$ \\
\hline Grip Strength $(\mathrm{kg})^{\mathrm{a}}$ & $.85(N=17)$ & $.84(N=17)$ & $.70(N=17)$ & $.82(N=17)$ \\
Movement Time $(\mathrm{s})^{\mathrm{a}}$ & $-.50(N=20)$ & $-.51(N=20)$ & $-.34(N=17)$ & $-.30(N=17)$ \\
Smoothness $(\mathrm{nmu})^{\mathrm{a}}$ & $-.16(N=20)$ & $-.21(N=20)$ & $-.06(N=17)$ & $-.02(N=17)$ \\
Max Hand Opening (mm) & $.51(N=16)$ & $.54(N=16)^{\mathrm{a}}$ & $.57(N=13)$ & $.56(N=13)$ \\
Max Velocity (mm/s) & $.75(N=20)$ & $.63(N=20)$ & $.30(N=17)$ & $.41(N=17)$ \\
Temporal pattern (\%) & $.07(N=16)$ & $.19(N=16)^{\mathrm{a}}$ & $-.07(N=13)$ & $-.13(N=13)$ \\
Trunk Displacement (mm) & $-.65(N=20)$ & $-.57(N=20)$ & $-.38(N=17)$ & $-.39(N=17)$ \\
Elbow Excursion (degrees) & $.81(N=20)$ & $.80(N=20)^{\mathrm{a}}$ & $.56(N=17)$ & $.68(N=17)$ \\
Wrist Excursion (degrees) & $.23(N=20)$ & $.09(N=20)$ & $.09(N=17)$ & $.12(N=17)$ \\
Shoulder Excursion - Elevation Plane (degrees) & $.26(N=20)$ & $.32(N=20)$ & $.45(N=17)$ & $.48(N=17)$ \\
Shoulder Excursion - Elevation Angle (degrees) & $-.15(N=20)$ & $-.16(N=20)$ & $.09(N=17)$ & $.10(N=17)$ \\
Shoulder Excursion - Axial Rotation (degrees) & $.57(N=20)$ & $.64(N=20)$ & $.58(N=17)$ & $.62(N=17)$ \\
\hline
\end{tabular}

Abbreviations: FM: Fugl-Meyer assessment, ARAT: Action Research Arm Test, MAL AOU: Motor Activity Log Amount of Use, MAL QOM: Motor Activity Log Quality of Movement, nmu: number of movement units.

Values shown are Pearson correlation coefficients, or Spearman's rho indicated with ${ }^{\text {a }}$. Significant correlations in bold. 


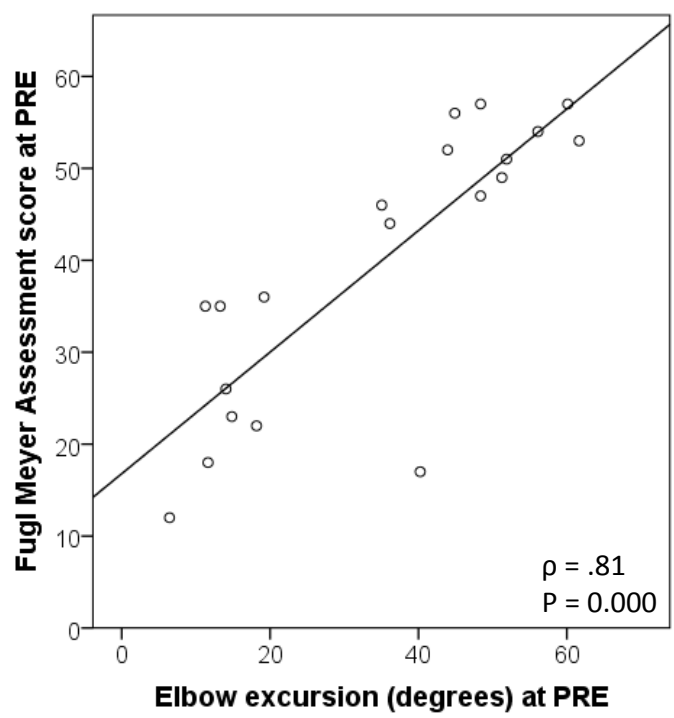

Figure 6.4 Scatterplot of Fugl-Meyer score with elbow excursion

\section{Multiple linear regression analyses}

Forward multiple regression revealed that one kinematic variable, elbow excursion, explained the largest amount of variance in the assessment of sensorimotor function, explaining $60.3 \%$ of the total variance in FM score. Elbow excursion and grip strength together explained $68.4 \%$ of the total variance in the assessment of activity capacity (ARAT), with a unique contribution of $12.0 \%(P=0.027)$ and $10.2 \%(P=0.039)$, respectively. In the models of MAL AOU and MAL QOM, grip strength was the only predictor that explained variance in self-perceived activity performance for both models, explaining $33.9 \%$ and $40.5 \%$ of the variance, respectively (Table 6.3 ).

\section{Training data}

At the PRE evaluation session, a significant difference between groups was found on smoothness, in favor of the experimental group (Table 6.4). Significant improvements over training were found for FM, MAL QOM and maximal velocity in the control group. The experimental group showed a significant decrease in elbow excursion after the training intervention. Significant differences between groups were found for training-induced changes on elbow excursion range, in favor of the control group (Table 6.4).

\section{Correlations training-induced changes}

In contrast with the correlations found at the PRE evaluation measurement, only a few significant correlations were observed in both groups when examining associations between training-induced changes in clinical and kinematic outcome measures on individual level via 
correlation analysis (Table 6.5). For the control group, improvements in activity capacity (ARAT) were strongly associated with improvements in maximal hand opening $(\rho=.89, \mathrm{P}=$ 0.041). For the experimental group, changes in activity capacity (ARAT) were associated with increased grip strength ( $\rho=.94, P=0.001)$. Remarkably, a change in self-perceived activity performance (MAL QOM) was associated negatively with changes in grip strength $(\rho=-.77$, $P=0.044)$, and MAL AOU was associated negatively with changes in wrist excursion ( $r=-.94$, $P=0.002)$ and changes in increased shoulder excursion elevation angle $(r=-.91, P=004)$.

Table 6.3 Results of multiple linear regression analyses (based on $\mathbf{N}=17$ )

\begin{tabular}{|c|c|c|c|c|c|c|}
\hline \multirow[t]{2}{*}{ Model } & \multirow{2}{*}{$\begin{array}{l}\text { Dependent } \\
\text { variable }\end{array}$} & \multirow{2}{*}{$\begin{array}{l}\text { Extracted predictors } \\
\text { in forward regression }\end{array}$} & \multicolumn{2}{|c|}{ Unstandardized Coefficients } & \multirow[t]{2}{*}{ Adjusted $\mathrm{R}^{2}$} & \multirow[t]{2}{*}{ Model P-value } \\
\hline & & & B & Std. Error & & \\
\hline \multirow[t]{2}{*}{1} & \multirow[t]{2}{*}{ FM } & Constant & 17.209 & 4.472 & \multirow{2}{*}{.603} & \multirow{2}{*}{.000} \\
\hline & & Elbow Excursion & .620 & .123 & & \\
\hline \multirow[t]{2}{*}{2} & \multirow[t]{2}{*}{ ARAT } & Constant & 1.682 & 5.773 & \multirow{2}{*}{.597} & \multirow{2}{*}{.000} \\
\hline & & Elbow Excursion & .790 & .159 & & \\
\hline \multirow[t]{3}{*}{3} & \multirow[t]{3}{*}{ ARAT } & Constant & .695 & 5.124 & \multirow{3}{*}{.684} & \multirow{3}{*}{.000} \\
\hline & & Elbow Excursion & .482 & .195 & & \\
\hline & & Grip Strength & .723 & .318 & & \\
\hline \multirow[t]{2}{*}{4} & \multirow[t]{2}{*}{ MAL AOU } & Constant & .833 & .376 & \multirow{2}{*}{.339} & \multirow{2}{*}{.008} \\
\hline & & Grip Strength & .062 & .020 & & \\
\hline \multirow[t]{2}{*}{5} & \multirow[t]{2}{*}{ MAL QOM } & Constant & .618 & .330 & \multirow{2}{*}{.405} & \multirow{2}{*}{.004} \\
\hline & & Grip Strength & .062 & .018 & & \\
\hline
\end{tabular}

Abbreviations: FM: Fugl-Meyer assessment, ARAT: Action Research Arm Test, MAL AOU: Motor Activity Log Amount of Use, MAL QOM: Motor Activity Log Quality of Movement

\section{DISCUSSION}

The current study investigated the relationships between an extensive set of objective movement execution kinematics, obtained from 20 chronic stroke patients during a functional reach and grasp task, grip strength and clinical outcome measures on sensorimotor function, activity capacity and self-perceived activity performance. This evaluation was repeated in 15 stroke patients after six weeks of arm and hand training at home, aimed at obtaining a more in-depth insight into the role of recovery versus compensation in technologysupported training. Almost all movement execution parameters showed strong or moderate relationships with sensorimotor function and activity capacity at the PRE measurement, with strongest correlations for grip strength and elbow excursion. The strong relationships seen during the PRE measurement were not transferable to relationships considering training-induced changes on individual level. 
Table 6.4 Mean values (SD) clinical outcomes and kinematic variables PRE and POST training

\begin{tabular}{|c|c|c|c|c|}
\hline & Group & PRE & POST & Mean change \\
\hline \multirow[t]{2}{*}{ FM score (maximal 66 points) } & $\operatorname{CON} N=8$ & $37.9(12.0)^{\mathrm{b}}$ & $40.1(12.6)^{b}$ & $2.3(1.8)$ \\
\hline & $\operatorname{EXP} N=7$ & $39.6(17.7)$ & $41.0(16.9)$ & $1.4(2.9)$ \\
\hline \multirow[t]{2}{*}{ ARAT score (maximal 57 points) } & $\operatorname{CON} N=8$ & $26.3(16.0)$ & $28.8(16.7)$ & $2.5(3.0)$ \\
\hline & $\operatorname{EXP} N=7$ & $32.6(21.3)$ & $32.6(21.5)$ & $0.0(1.4)$ \\
\hline \multirow[t]{2}{*}{ MAL AOU (0-5) } & $\operatorname{CON} N=8$ & $1.9(1.2)$ & $2.3(1.3)$ & $0.4(0.6)$ \\
\hline & $\operatorname{EXP} N=7$ & $1.9(1.1)$ & $1.9(1.2)$ & $0.0(0.2)$ \\
\hline \multirow[t]{2}{*}{ MAL QOM (0-5) } & $\operatorname{CON} N=8$ & $1.7(1.1)^{\mathrm{b}}$ & $2.0(1.3)^{b}$ & $0.3(0.4)$ \\
\hline & $\operatorname{EXP} N=7$ & $1.7(1.0)$ & $1.8(1.0)$ & $0.0(0.2)$ \\
\hline \multirow[t]{2}{*}{ Grip strength (kg) } & $\operatorname{CON} N=8$ & $14.1(8.4)$ & $14.5(9.2)$ & $0.4(2.2)$ \\
\hline & $\operatorname{EXP} N=7$ & $18.4(14.7)$ & $19.3(14.8)$ & $0.9(4.4)$ \\
\hline \multirow[t]{2}{*}{ Movement Time (s) } & $\operatorname{CON} N=8$ & $4.5(3.3)$ & $3.7(3.1)$ & $-0.9(1.5)$ \\
\hline & $\operatorname{EXP} N=7$ & $2.3(0.7)$ & $2.1(0.7)$ & $-0.3(0.6)$ \\
\hline \multirow[t]{2}{*}{ Smoothness (nmu) } & $\operatorname{CON} N=8$ & $10.5(8.1)^{\mathrm{a}}$ & $8.6(8.0)$ & $-2.0(2.8)$ \\
\hline & $\operatorname{EXP} N=7$ & $5.3(2.3)^{a}$ & $5.5(2.0)$ & $0.2(2.2)$ \\
\hline \multirow[t]{2}{*}{ Max hand opening (mm) } & $\operatorname{CON} N=5$ & $160.0(24.0)$ & $162.8(20.9)$ & $2.8(9.9)$ \\
\hline & $\operatorname{EXP} N=5$ & $174.1(17.5)$ & $173.4(9.1)$ & $-0.7(10.7)$ \\
\hline \multirow[t]{2}{*}{ Max velocity (mm/s) } & $\operatorname{CON} N=8$ & $375.3(132.7)^{b}$ & $470.8(180.7)^{b}$ & $95.5(70.5)$ \\
\hline & $\operatorname{EXP} N=7$ & $505.5(182.7)$ & $509.3(140.8)$ & 3.7 (95.5) \\
\hline \multirow[t]{2}{*}{ Temporal pattern (\%) } & $\operatorname{CON} N=5$ & $137.6(84.7)$ & $137.3(42.5)$ & $-0.4(44.8)$ \\
\hline & $\operatorname{EXP} N=5$ & $187.2(96.5)$ & $114.5(59.2)$ & $-72.7(80.5)$ \\
\hline \multirow[t]{2}{*}{ Trunk displacement (mm) } & $\operatorname{CON} N=8$ & $88.6(55.3)$ & $108.1(70.2)$ & $19.5(49.1)$ \\
\hline & $\operatorname{EXP} N=7$ & $67.7(49.8)$ & $76.5(54.8)$ & $8.8(38.2)$ \\
\hline \multirow[t]{2}{*}{ Elbow excursion (degrees) } & $\operatorname{CON} N=8$ & $29.1(19.3)$ & $30.4(17.5)$ & $1.3(4.2)^{c}$ \\
\hline & $\operatorname{EXP} N=7$ & $39.4(17.2)^{b}$ & $36.2(15.6)^{b}$ & $-3.1(3.3)^{c}$ \\
\hline \multirow[t]{2}{*}{ Wrist excursion (degrees) } & $\operatorname{CON} N=8$ & $24.0(8.8)$ & $25.5(8.4)$ & $1.5(5.6)$ \\
\hline & $\operatorname{EXP} N=7$ & $20.3(13.0)$ & $23.0(11.1)$ & $2.7(6.5)$ \\
\hline \multirow[t]{2}{*}{ Shoulder Excursion - Elevation Plane (degrees) } & $\operatorname{CON} N=8$ & $32.8(16.8)$ & $30.9(19.4)$ & $-1.9(6.7)$ \\
\hline & $\operatorname{EXP} N=7$ & $36.5(12.3)$ & $29.8(7.3)$ & $-6.7(11.4)$ \\
\hline \multirow[t]{2}{*}{ Shoulder Excursion - Elevation Angle (degrees) } & $\operatorname{CON} N=8$ & $17.3(4.8)$ & $19.3(7.1)$ & $2.0(3.7)$ \\
\hline & $\operatorname{EXP} N=7$ & $13.9(8.0)$ & $13.5(6.1)$ & $-0.4(7.6)$ \\
\hline \multirow[t]{2}{*}{ Shoulder Excursion - Axial Rotation (degrees) } & $\operatorname{CON} N=8$ & $40.1(20.0)$ & $38.7(20.3)$ & $-1.4(9.0)$ \\
\hline & $\operatorname{EXP} N=7$ & $43.4(19.3)$ & $35.3(13.4)$ & $-8.1(11.3)$ \\
\hline
\end{tabular}

Abbreviations: FM: Fugl-Meyer assessment, ARAT: Action Research Arm Test, MAL AOU: Motor Activity Log Amount of Use, MAL QOM: Motor Activity Log Quality of Movement, CON: control group, EXP: experimental group, PRE: evaluation measurement pre-training, POST: evaluation measurement post-training, nmu: number of movement units.

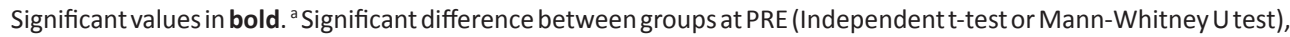
b Significant difference within groups (PRE vs POST: Paired t-test or Wilcoxon Signed Ranks, ${ }^{\mathrm{b}}$ Significant change between groups (Mean change: Independent t-test or Mann-Whitney U test). 
Table 6.5 Correlation coefficients training induced changes clinical outcomes measures and kinematic outcomes for the control group (left) and experimental group (right)

\begin{tabular}{|c|c|c|c|c|c|c|c|c|c|}
\hline & \multicolumn{4}{|c|}{ Control group $(N=8)$} & & \multicolumn{4}{|c|}{ Experimental group $(N=7)$} \\
\hline & FM & ARAT $^{\mathrm{a}}$ & $\begin{array}{l}\text { MAL } \\
\text { AOU }\end{array}$ & $\begin{array}{l}\text { MAL } \\
\text { QOM }\end{array}$ & & FM & ARAT & $\begin{array}{l}\text { MAL } \\
\text { AOU }\end{array}$ & $\begin{array}{l}\text { MAL } \\
\text { QOM }\end{array}$ \\
\hline Grip Strength (kg) & -.42 & .66 & .33 & -.37 & Grip Strength (kg) ${ }^{a}$ & .75 & .94 & .45 & -.77 \\
\hline $\mathrm{MT}(\mathrm{s})^{\mathrm{a}}$ & .05 & -.57 & -.29 & .02 & MT (s) & .30 & .32 & -.28 & -.32 \\
\hline MS (nmu) & .47 & -.20 & .22 & .56 & MS (nmu) & .07 & .07 & .10 & .10 \\
\hline Max HO $(\mathrm{mm})^{\mathrm{b}}$ & .14 & .89 & .42 & .59 & Max HO $(\mathrm{mm})^{\mathrm{b}}$ & -.79 & -.75 & .23 & .80 \\
\hline Max Velocity $(\mathrm{mm} / \mathrm{s})$ & .02 & .68 & .43 & .25 & Max Velocity $(\mathrm{mm} / \mathrm{s})$ & .61 & .27 & -.27 & -.40 \\
\hline $\begin{array}{l}\text { Temporal pattern } \\
(\%)^{b}\end{array}$ & -.17 & .11 & -.35 & -.09 & $\begin{array}{l}\text { Temporal pattern } \\
(\%)^{\mathrm{b}}\end{array}$ & -.39 & -.11 & .26 & .30 \\
\hline TD $(\mathrm{mm})^{\mathrm{a}}$ & .11 & -.43 & .24 & -.14 & TD (mm) & -.08 & .00 & -.52 & -.26 \\
\hline EE (degrees) & -.03 & .15 & .32 & .12 & EE (degrees) & -.57 & -.59 & .41 & .72 \\
\hline WE (degrees) & .25 & .16 & .11 & .10 & WE (degrees) & .31 & .11 & -.94 & -.55 \\
\hline EP (degrees) & .01 & .15 & -.39 & -.09 & EP (degrees) & .42 & .40 & .56 & -.13 \\
\hline EA (degrees) & -.25 & .09 & -.21 & -.10 & EA (degrees) & .42 & .26 & -.91 & -.61 \\
\hline AR (degrees) & -.22 & .11 & .10 & .09 & AR (degrees) & .66 & .54 & .29 & -.31 \\
\hline
\end{tabular}

Abbreviations: FM: Fugl-Meyer assessment (maximum 66), ARAT: Action Research Arm Test (maximum 57), MAL AOU: Motor Activity Log Amount of Use (0-5), MAL QOM: Motor Activity Log Quality of Movement (0-5), MT: movement time, MS: movement smoothness, nmu: number of movement units, HO: hand opening, TD: forward trunk displacement, EE: elbow flexion and extension excursion, WE: wrist flexion and extension excursion, EP: elevation plane, EA: elevation angle, AR: axial rotation.

Significant correlations in bold. a Spearman's rho (otherwise Pearson Correlation), ${ }^{\text {b }}$ Correlations based on $N=5$.

\section{Cross-sectional measurement PRE training}

Previous studies demonstrated high associations among clinical outcome measures, such as FM and ARAT. ${ }^{35-37}$ Associations of kinematic outcomes with clinical outcome measures have also been examined, ${ }^{17-19}$ but with a limited set of movement execution parameters. In the current study, strong relationships were found for movement execution parameters and grip strength with sensorimotor function and activity capacity. The relationships with selfperceived activity performance in daily life were weaker, which is comparable to previous research. ${ }^{17}$ In the current study, elbow excursion was the most significant contributor to the variance in FM score (60.3\%), and a combination of elbow excursion and grip strength explained the majority of variance $(68.4 \%)$ in ARAT score.

Other studies indicated that movement smoothness and total movement time, together with compensatory trunk displacement associated best with ARAT ${ }^{17}$ and trunk displacement with $\mathrm{FM}^{18}$. However, outcomes involving hand grip strength ${ }^{17,18}$ and elbow excursion (range of motion) ${ }^{17}$ were not included in these above-mentioned studies. We also found moderate correlations for FM and ARAT with movement time and trunk displacement, but elbow excursion and grip strength showed stronger associations in the regression models 
when all of these parameters were considered. It can be argued that compensatory trunk displacement might be reflected in decreased elbow excursion. Indeed, initial inspection of the data for multicollinearity showed a moderate negative correlation between trunk displacement and elbow excursion, which supports these findings in the context of previous research.

The present findings on PRE correlations and corresponding determinants strongly suggest that it is valuable for treatment applications in neurorehabilitation after stroke to consider targeting at least elbow excursion and hand grip strength, i.e., both reaching and grasping. This is in agreement with studies indicating that task-specific, functional exercises have a high potential to stimulate functional improvements. ${ }^{38}$

\section{Training data}

In line with this, we applied a newly designed training intervention aimed at both reaching and grasping within the SCRIPT project. ${ }^{20}$ Nevertheless, functional improvements as assessed using clinical outcome measures after the training interventions were most pronounced, yet limited, in the control group performing home exercises related to activities of daily living from a booklet. ${ }^{22}$ The additional in-depth analysis of changes in kinematic outcome measures in the current study revealed improvements in maximal hand movement velocity in the control group only. In the experimental group, elbow excursion showed a remarkable decrease. Although unexpected, this may point towards an association with the lack of improvement in sensorimotor function or activities in the experimental group, considering the correlation outcomes at PRE.

Overall, improvements in sensorimotor function, activity limitations or movement execution parameters, if any, were modest at best. This makes it difficult to fully address the aim of whether mechanisms of recovery and/or compensation were involved in this case. Some indications can be derived from the direction of improvement. The control group showed an improvement in sensorimotor function (FM) and activity performance (MAL QOM) together with an improved hand movement velocity. However, the experimental group reduced their ability to reach as reflected by a decrease in elbow excursion, which could be a sign of increased compensation. However, since only limited changes on group level occurred in the current sample after these two particular interventions, this does not reflect conclusive evidence.

\section{Correlations training-induced changes}

It is recognized that individual data display large variations between stroke patients, also in terms of amount of change after a (technology-supported) intervention. ${ }^{39}$ Therefore, correlation analysis of change scores, considering individual cases, might reveal a more 
realistic picture in understanding which factors may contribute to functional improvements and in which patients. In this regard, the findings in the current sample suggest that improvements in activity capacity after training were most associated with improvements in hand function (either in terms of range of motion or strength). However, the absolute differences were small.

Surprisingly, the self-perceived performance measure showed different results than the capacity measure. A change in MAL after training was negatively correlated with changes in grip strength, wrist excursion and shoulder excursion elevation angle in the experimental group, which is difficult to interpret in context of the current study. This is possibly because of the subjective nature of the MAL, or the MAL measuring other constructs than actual performance. Potentially, participants could relate this to other qualities of movement. Further, improvements on function, capacity and self-perceived performance might not occur simultaneously..$^{40}$

In general, chronic stroke patients are thought to benefit most from task-specific interventions, ${ }^{41}$ involving both reaching and grasping, which was the approach for both training interventions. ${ }^{22}$ The fact that changes in activity capacity were associated with predominantly hand function improvements, whereas no substantial hand function improvements were evident on group level after technology-supported training, suggests that this intervention apparently did not sufficiently target hand function. Although the intervention with the current sample did not provide the desired information, we expect that the current analysis approach is useful to give more insight into the underlying mechanisms of recovery and compensation after stroke. In addition, it provides more specific directions for design of (technology-supported) interventions for arm and hand function. We recommend targeted interventions, particularly addressing functional movements involving both reaching (elbow excursion) and hand function (grip strength or hand opening) after stroke. Moreover, evaluation measurements should be included that address these particular endpoints. This is in line with expert consensus gained for guidelines facilitating standardized assessment of such interventions, which proposed inclusion of technologies as assessment tools besides clinical scales. ${ }^{10}$ This consensus approach highlighted the need for more information about useful data derived from such technological methods, for which the current findings provide a first indication.

\section{Limitations}

A limitation of our study concerns the relatively small sample size, $N=20$ for the correlations and multiple linear regression analyses pre-training and $\mathrm{N}=15$ for the data on traininginduced changes. Elbow excursion and grip strength together were the most important predictors in the model of ARAT. For multiple linear regression models, a minimum of ten 
observations per predictor variable will generally allow good estimates. ${ }^{42}$ Since the model of ARAT was based on only 17 participants, there is a possibility of overfitting of the model, which should be taken into account when interpreting the results. Further, the current training intervention was performed with chronic stroke patients at home, with somewhat more impaired participants than previous studies measuring correlations between kinematic and clinical outcomes, ${ }^{17}$ which limits the generalizability of the results to other patient groups in other settings. In practice, these interventions should be considered at an earlier stage after stroke, where larger treatment effects would be expected in the subacute phase. ${ }^{43}$

\section{CONCLUSION}

Moderate to strong relationships of movement execution parameters and grip strength with FM and ARAT were found, with strongest contributions for grip strength and elbow excursion. The findings imply that the inclusion of both reaching (elbow excursion) with hand function (grip strength or hand opening) might be valuable targets for (technologysupported) treatment applications to stimulate functional improvements after stroke. To which extent this was successful in the current technology-supported intervention remains unclear due to limited training-induced changes, but it does provide directions for design of (technology-supported) interventions for arm and hand function. We recommend targeted interventions addressing functional movements involving both arm and hand movements simultaneously, and including objective measures of elbow excursion and hand function (grip strength and hand opening) to evaluate their effects.

\section{REFERENCES}

1. Nichols-Larsen DS, Clark PC, Zeringue A, Greenspan A and Blanton S. Factors influencing stroke survivors' quality of life during subacute recovery. Stroke; a journal of cerebral circulation. 2005; 36: 1480-4.

2. Kitago T and Krakauer JW. Chapter 8 - Motor learning principles for neurorehabilitation. In: Michael PB and David CG, (eds.). Handb Clin Neurol. Elsevier, 2013, p. 93-103.

3. Schaechter JD. Motor rehabilitation and brain plasticity after hemiparetic stroke. Progress in Neurobiology. 2004; 73: 61-72.

4. Kwakkel G. Impact of intensity of practice after stroke: issues for consideration. Disabil Rehabil. 2006; 28: 823-30.

5. Mehrholz J, Pohl M, Platz T, Kugler J and Elsner B. Electromechanical and robot-assisted arm training for improving activities of daily living, arm function, and arm muscle strength after stroke. Cochrane Database Syst Rev. 2015; 11: CD006876.

6. Maciejasz P, Eschweiler J, Gerlach-Hahn K, Jansen-Troy A and Leonhardt S. A survey on robotic devices for upper limb rehabilitation. Journal of Neuroengineering and Rehabilitation. 2014; 11: 3.

7. Kwakkel G, Kollen BJ and Krebs HI. Effects of robot-assisted therapy on upper limb recovery after stroke: a systematic review. Neurorehabil Neural Repair. 2008; 22: 11121.

8. Sivan M, O'Connor RJ, Makower S, Levesley M and Bhakta B. Systematic review of outcome measures used in the evaluation of robot-assisted upper limb exercise in 
stroke. J Rehabil Med. 2011; 43: 181-9.

9. Alt Murphy M, Resteghini C, Feys P and Lamers I. An overview of systematic reviews on upper extremity outcome measures after stroke. BMC neurology. 2015; 15: 29.

10. Hughes $A M$, Boucas $S B$, Burridge $J H$, et al. Evaluation of upper extremity neurorehabilitation using technology: a European Delphi consensus study within the EU COST Action Network on Robotics for Neurorehabilitation. J Neuroeng Rehabil. 2016; 13: 86.

11. WHO. International Classification of Functioning, Disability and Health (ICF). Geneva: WHO, 2001.

12. Levin MF, Kleim JA and Wolf SL. What do motor "recovery" and "compensation" mean in patients following stroke? NeurorehabilNeural Repair. 2009; 23: 313-9.

13. Cirstea MC and Levin MF. Compensatory strategies for reaching in stroke. Brain. 2000; 123 ( Pt 5): 940-53.

14. van Kordelaar J, van Wegen EE, Nijland $\mathrm{RH}$, et al. Assessing longitudinal change in coordination of the paretic upper limb using on-site 3-dimensional kinematic measurements. Phys Ther. 2012; 92: 142-51.

15. Kwakkel G, Kollen B and Lindeman E. Understanding the pattern of functional recovery after stroke: facts and theories. Restor Neurol Neurosci. 2004; 22: 281-99.

16. Langhorne P, Bernhardt J and Kwakkel G. Stroke rehabilitation. Lancet. 2011; 377: 1693702.

17. Alt Murphy M, Willen C and Sunnerhagen KS. Movement kinematics during a drinking task are associated with the activity capacity level after stroke. Neurorehabilitation and Neural Repair. 2012; 26: 1106-15.

18. Subramanian SK, Yamanaka J, Chilingaryan G and Levin MF. Validity of movement pattern kinematics as measures of arm motor impairment poststroke. Stroke; a journal of cerebral circulation. 2010; 41: 2303-8.

19. Chen HL, Lin KC, Liing RJ, Wu CY and Chen CL. Kinematic measures of Arm-trunk movements during unilateral and bilateral reaching predict clinically important change in perceived arm use in daily activities after intensive stroke rehabilitation. J Neuroeng Rehabil. 2015; 12: 84.

20. Amirabdollahian F, Ates S, Basteris A, et al. Design, development and deployment of a hand/wrist exoskeleton for home-based rehabilitation after stroke - SCRIPT project. Robotica. 2014; 32: 1331-46.

21. Nijenhuis SM, Prange GB, Stienen AHA, Buurke JH and Rietman JS. Direct effect of a dynamic wrist and hand orthosis on reach and grasp kinematics in chronic stroke. IEEE International Conference on Rehabilitation Robotics (ICORR), 2015. IEEE, 2015, p. 404-9.

22. Nijenhuis SM, Prange-Lasonder GB, Stienen AH, Rietman JS and Buurke JH. Effects of training with a passive hand orthosis and games at home in chronic stroke: a pilot randomised controlled trial. Clin Rehabil. 2017; 31: 207-16.

23. Fugl-Meyer AR, Jaasko L, Leyman I, Olsson S and Steglind S. The post-stroke hemiplegic patient. 1. A method for evaluation of physical performance. Scand J Rehabil Med. 1975; 7: 13-31.

24. Deakin A, Hill H and Pomeroy VM. Rough Guide to the Fugl-Meyer Assessment: Upper limb section. Physiotherapy. 2003; 89: 751-63.

25. Lyle RC. A performance test for assessment of upper limb function in physical rehabilitation treatment and research. Int J Rehabil Res. 1981; 4: 483-92.

26. Yozbatiran N, Der-Yeghiaian L and Cramer SC. A standardized approach to performing the action research arm test. Neurorehabil Neural Repair. 2008; 22: 78-90.

27. van der Lee JH, Beckerman $\mathrm{H}$, Knol DL, de Vet $\mathrm{HC}$ and Bouter LM. Clinimetric properties of the motor activity log for the assessment of arm use in hemiparetic patients. Stroke. 
2004; 35: 1410-4.

28. Mathiowetz V, Kashman N, Volland G, Weber K, Dowe M and Rogers S. Grip and pinch strength: normative data for adults. Arch Phys Med Rehabil. 1985; 66: 69-74.

29. Wu G, van der Helm FC, Veeger HE, et al. ISB recommendation on definitions of joint coordinate systems of various joints for the reporting of human joint motion--Part II: shoulder, elbow, wrist and hand. J Biomech. 2005; 38: 981-92.

30. Metcalf CD, Notley SV, Chappell PH, Burridge JH and Yule VT. Validation and application of a computational model for wrist and hand movements using surface markers. IEEE Trans Biomed Eng. 2008; 55: 1199-210.

31. Alt Murphy M, Willen $C$ and Sunnerhagen KS. Kinematic variables quantifying upperextremity performance after stroke during reaching and drinking from a glass. Neurorehabilitation and Neural Repair. 2011; 25: 71-80.

32. Doeringer JA and Hogan N. Serial processing in human movement production. Neural Netw. 1998; 11: 1345-56.

33. Krabben $\mathrm{T}$, Prange $\mathrm{GB}$, Molier $\mathrm{BI}$, et al. Influence of gravity compensation training on synergistic movement patterns of the upper extremity after stroke, a pilot study. $J$ Neuroeng Rehabil. 2012; 9: 44.

34. Luft AR, McCombe-Waller S, Whitall J, et al. Repetitive bilateral arm training and motor cortex activation in chronic stroke: a randomized controlled trial. JAMA. 2004; 292: 1853-61.

35. Hoonhorst $\mathrm{MH}$, Nijland $\mathrm{RH}$, van den Berg JS, Emmelot $\mathrm{CH}$, Kollen BJ and Kwakkel G. How Do Fugl-Meyer Arm Motor Scores Relate to Dexterity According to the Action Research Arm Test at 6 Months Poststroke? Arch Phys Med Rehabil. 2015; 96: 1845-9.

36. Rabadi $\mathrm{MH}$ and Rabadi FM. Comparison of the action research arm test and the FuglMeyer assessment as measures of upper-extremity motor weakness after stroke. Arch Phys Med Rehabil. 2006; 87: 962-6.

37. Wei XJ, Tong KY and Hu XL. The responsiveness and correlation between Fugl-Meyer Assessment, Motor Status Scale, and the Action Research Arm Test in chronic stroke with upper-extremity rehabilitation robotic training. Int J Rehabil Res. 2011; 34: 349-56.

38. Timmermans AAA, Geers RPJ, Franck JA, et al. T-TOAT: A method of task-oriented arm training for stroke patients suitable for implementation of exercises in rehabilitation technology. 2009 leee 11th International Conference on Rehabilitation Robotics, Vols 1 and 2. 2009, p. 114-8.

39. Nijenhuis SM, Prange GB, Amirabdollahian F, et al. Feasibility study into self-administered training at home using an arm and hand device with motivational gaming environment in chronic stroke. Journal of Neuroengineering and Rehabilitation. 2015; $12: 89$.

40. Michielsen ME, de Niet M, Ribbers GM, Stam HJ and Bussmann JB. Evidence of a logarithmic relationship between motor capacity and actual performance in daily life of the paretic arm following stroke. J Rehabil Med. 2009; 41: 327-31.

41. Kwakkel G, Veerbeek JM, van Wegen EE and Wolf SL. Constraint-induced movement therapy after stroke. Lancet Neurol. 2015; 14: 224-34.

42. Babyak MA. What you see may not be what you get: a brief, nontechnical introduction to overfitting in regression-type models. Psychosom Med. 2004; 66: 411-21.

43. Kwakkel G, Kollen BJ and Wagenaar RC. Long term effects of intensity of upper and lower limb training after stroke: a randomised trial. J Neurol Neurosurg Psychiatry. 2002; 72: 473-9. 



\section{Chapter 7}

Feasibility of a second iteration wrist and hand supported training system for self-administered training at home in chronic stroke

Nijenhuis SM, Prange-Lasonder GB, Amirabdollahian F, Infarinato F, Buurke JH, Rietman JS Proceedings of 8th International Conference on eHealth, Telemedicine, and Social Medicine 2016; p.51-56 


\section{ABSTRACT}

Telerehabilitation allows continued rehabilitation at home after discharge. The use of rehabilitation technology supporting wrist and hand movements within a motivational gaming environment could enable patients to train independently and ultimately serve as a way to increase the dosage of practice. This has been previously examined in the European Supervised Care \& Rehabilitation Involving Personal Telerobotics (SCRIPT) project using a first prototype, showing potential feasibility, although several usability issues needed further attention. The current study examined feasibility and clinical changes of a second iteration training system, involving an updated wrist and hand supporting orthosis and larger variety of games with respect to the first iteration. The paper is relevant for the conference, reporting a new telemedicine service, combining physical orthotic support with remote offline supervision, for telerehabilitation at home after stroke. Nine chronic stroke patients with impaired arm and hand function were recruited to use the training system at home for six weeks. Evaluation of feasibility and arm and hand function were assessed before and after training. Median weekly training duration was 113 minutes. Participants accepted the six weeks of training (median Intrinsic Motivation Inventory $=4.4$ points and median System Usability Scale $=73 \%$ ). After training, significant improvements were found for the Fugl-Meyer assessment, Action Research Arm Test and self-perceived amount of arm and hand use in daily life. These findings indicate that technology-supported arm and hand training can be a promising tool for self-administered practice at home after stroke. 


\section{INTRODUCTION}

Stroke is one of the most common causes of adult disability. Even in the chronic phase, motor problems still persist in the majority of stroke patients, ${ }^{1}$ leading to difficulties in performing activities of daily living independently. Motor problems that persist in the chronic phase may be partly due to learned nonuse of the affected upper limb. ${ }^{2}$ Stimulating the use of the affected upper extremity has shown to overcome the learned nonuse. ${ }^{3}$

Technology has the potential to support rehabilitation since it can provide high-intensive, repetitive, task-specific, interactive treatment of the impaired upper extremity. Besides, it has the potential to accurately quantify therapy and monitor patients' progress, while also providing immediate feedback to patients, as well as therapists. Rehabilitation robotics has been shown to be as effective as conventional rehabilitation for the hemiparetic arm. ${ }^{4-7}$ Most research so far has shown significant improvements in upper limb motor function, although evidence of the transfer of robotic training effects to activities in daily life remains limited, as is observed for most interventions in stroke rehabilitation, including conventional therapy. ${ }^{8}$ To maximize independent use of the upper extremity in daily life, it is important to include functional movements of both the proximal and distal arm and hand into poststroke training, since a generalization effect to improvements on the entire upper extremity was found. 9,10

Most robotic devices are mainly suitable for the clinical setting with direct supervision of a therapist. ${ }^{5}$ A next step would be to provide such systems at home, to enable selfadministered practice of the arm and hand after stroke. ${ }^{11}$ This is especially interesting since an increasing number of stroke survivors is expected, which will result in increased demands on healthcare systems. ${ }^{12}$ New ways of providing healthcare services, such as teleconsultation and remote monitoring and treatment in the patient's home are therefore needed.

Telemedicine systems for upper extremity exercise showed promising results in improving health of stroke patients. ${ }^{13}$ In addition, healthcare professionals and participants reported good levels of satisfaction and acceptance of telerehabilitation interventions. ${ }^{13,14}$ This is in line with the precursor of the current study, using a passive device with three motivational rehabilitation games for arm and hand training at home, ${ }^{15,16}$ showing that the training was motivational which was underlined by an average weekly training duration of $105 \pm$ 66 minutes. Usability showed potential, although several usability issues needed further attention. Clinical evaluations showed modest changes in arm and hand function. ${ }^{15}$

In the current study, we expanded this research by using a next iteration of the developed training system. Lessons learned regarding usability findings, therapeutic benefit and practical issues which were obtained in the first iteration of patient measurements with the 
SCRIPT system ${ }^{15,16}$ have been taken into account for the design of the second prototype. An updated passive wrist and hand orthosis with improved user interface including nine exercise games was provided. The new games further focused on functional exercises incorporating versatile grasping gestures. The second prototype was evaluated with a new group of chronic stroke patients. The objective of the current study was to examine feasibility (user acceptance and adherence) and clinical changes in arm and hand function of a second generation technology-supported arm and hand training system at home in chronic stroke.

\section{Methods}

\section{Participants}

Participants were recruited from the Roessingh Rehabilitation center, Enschede, the Netherlands and IRCCS San Raffaele Pisana, Rome, Italy. Participants were eligible for inclusion if they (1) had a stroke between 6 months and 5 years ago; (2) were between 18 and 80 years of age; (3) had limited arm and hand function because of the stroke, but having at least active control of $15^{\circ}$ elbow flexion and having active finger flexion of at least a quarter of the passive range of motion; (4) were living at home and having internet access; (5) were able to understand and follow instructions; (6) had no additional orthopedic, neurological, or rheumatologic disease of the upper extremity; and (7) no severe neglect or uncorrected visual impairments. All participants provided written informed consent before participation. The study protocol was approved by the local medical ethics committees (Medisch Spectrum Twente, Enschede, the Netherlands and the IRCCS San Raffaele Pisana ethics committee, Rome, Italy).

\section{Study design}

This feasibility study has a longitudinal design. The participants received six weeks of selfadministered technology-supported training for the arm and hand at home. Evaluation of arm and hand function was based on a baseline measurement pre-training and an evaluation measurement within one week post-training, performed at the research lab of the rehabilitation center.

\section{Training intervention}

Participants used a technology-supported training system, ${ }^{16}$ which consisted of a slightly adapted version of the SCRIPT dynamic wrist and hand orthosis, ${ }^{17}$ a mobile arm support (SaeboMAS, Saebo Inc., Charlotte NC, USA) and a computer with webcam and touchscreen displaying exercise games (Figure 7.1). The mobile arm support was used to support the weight of the proximal arm. The wrist and hand orthosis is a passive exoskeleton worn on the forearm and hand, customized to the hand size of each participant. It provides extension forces to the wrist and fingers via passive leaf springs and elastic tension cords. ${ }^{17}$ The orthosis was equipped with sensors to measure joint excursions of the wrist and hand, 
which allowed control of nine exercise games. A green marker placed on the hand plate of the orthosis was used to track the location of the orthosis by means of a camera placed on top of the screen to incorporate translational movements of the arm.

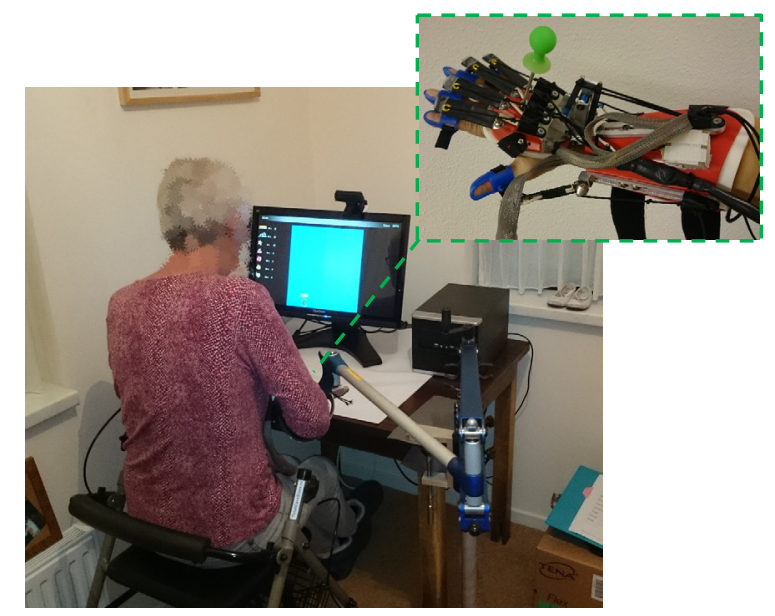

Figure 7.1 Training system at home

The exercise games consisted of various difficulty categories, to match the progress of individual participants. The categories were classified in a game difficulty schedule, ranked according to increasing complexity. Complexity was higher when a game required multiple movement planes (from 1D to 3D), involved a higher number of gestures to control the game, movements with progression from proximal to distal movements or gross to fine manipulation. The gestures needed to control the games were hand opening and closing, wrist flexion and extension, forearm pronation and supination, and reaching forwards, backwards and moving left or right. For hand opening and closing we could distinguish a general grasp, cylinder grasp, tripod grasp and lateral grasp, which have been shown to be reliable hand postures which could be recognized during performance of rehabilitation games. ${ }^{18}$ Translational movements of the hand were integrated with wrist and hand movements (e.g., moving the hand to a target, grasping, transferring to a different target, releasing) to emphasize functional, task-specific movements.

The training environment was available within a motivational user interface including feedback on performance, which was displayed on the touchscreen. The general recommendation for training was about 30 minutes per day, six days a week. Participants could train at the time of the day they preferred and were allowed to practice additionally if they wished to. A trained healthcare professional followed the participants' training progress remotely, offline, via another user interface available on a secured website and provided feedback by means of sending motivational messages when training duration 
was low. In addition, the healthcare professional visited each participant once per week to ensure competence with the training system, informally monitor progress, and to answer potential questions. Based on performance of the exercise games, in addition to ranking on the game difficulty schedule, the healthcare professional decided if a participant could move up to the next category of games. The professional adjusted the training program for the participants remotely.

\section{Evaluation \\ User experience}

The frequency and duration of training were automatically stored within the system and displayed in the user interface. The total minutes of training per week were counted to provide the total weekly training duration. These weekly training durations of all six weeks of training were used to calculate the average amount of practice over six weeks.

Motivation during training was measured using the Intrinsic Motivation Inventory (IMI) questionnaire.$^{19}$ It provides qualitative information about the content and level of motivation that a participant experienced during the training period (maximum score $=7$ ). A higher score represents higher motivation during training, with a neutral score of four.

The System Usability Scale (SUS) is a 10-item scale providing a global view of subjective usability. ${ }^{20,} 21$ The questions were scored on a 5-point Likert scale ranging from 'strongly agree' to 'strongly disagree'. Scores are translated to $0-100 \%$, with a higher score representing better usability. Interventions with scores in the 90 s are exceptional, scores in the 70 s and 80 s are promising, and with SUS scores below 50 one can be almost certain that the intervention will have usability difficulties in the field. The SUS and IMI were completed during the post-evaluation measurement only.

\section{Arm and hand function tests}

Clinical tests were used to quantify general arm function before and after the training. The scales used are valid, standardized assessments, which were performed according to their specific test protocols. The upper extremity part of the Brunnstrom Fugl-Meyer assessment (FM) evaluates motor status and the degree of synergy-development in the arm (maximum score $=66$ ). ${ }^{22}$ Separate scores were calculated for proximal (maximum $=42$ ) and distal components of the FM (maximum = 24).

The Action Research Arm Test (ARAT) evaluates coordination, dexterity and upper extremity function on the subtests grasp, grip, pinch, and gross arm movement (maximum score = 57). ${ }^{23}$ 
The Motor Activity Log (MAL) is a semi-structured interview for hemiparetic stroke patients to assess the perceived use of their paretic arm and hand (amount of use (AOU) and quality of movement (QOM)) during activities of daily living (maximum score $=5$ per subsection). ${ }^{24}$

The Stroke Impact Scale (SIS) was used to assess changes in function, activity and participation following stroke. The questionnaire assesses eight domains related to function, activities and participation. Each domain score has a range of zero to hundred percent, with a higher score indicating better quality of life. ${ }^{25}$

\section{Statistical analyses}

Statistical analyses were performed with IBM SPSS Statistics 19 for Windows. Outcomes were non-parametrically tested for statistical significance due to the small sample size. Descriptive statistical methods (median with interquartile range (IQR)) were used to describe the participant characteristics and all outcome measures. Outcomes of each clinical scale were compared between both evaluation measurements using the Wilcoxon signed rank test. The level for significance was set at $\alpha \leq .05$.

\section{RESULTS}

\section{Participants}

Nine participants (six in the Netherlands, three in Italy) were included in the study. Two participants were lost to the study. One because of personal problems not related to this study and one having recurrent technical problems with the system. The characteristics of the remaining seven participants are shown in Table 7.1.

Table 7.1 Participant characteristics

\begin{tabular}{lc}
\hline & $\boldsymbol{N}=\mathbf{7}^{\text {a }}$ \\
\hline Sex & 5 male / 2 female \\
Age & $57(44-67)$ years \\
Months post stroke & $21(9-33)$ \\
Type of stroke & 5 infarction / 2 hemorrhage \\
Affected body side & 4 left / 3 right \\
Dominant arm & 0 left / 7 right \\
Fugl-Meyer Assessment score (maximal 66 points) & 37 (30 - 45) \\
Action Research Arm Test score (maximal 57 points) & 26 (21 - 28) \\
Stroke severity & 6 moderate / 1 severe \\
\hline
\end{tabular}

${ }^{a}$ Results are shown as absolute numbers or median (interquartile range) 


\section{User experience}

The participants actually used the system, but with a large amount of variation between and within individuals (Figure 7.2). Median weekly training duration for the group, averaged over six weeks, was 113 (IQR 69-158) minutes. One participant (E01) exceeded the advised training duration of 180 minutes per week once, and two other participants (D08 and E03) exceeded the advised duration several times.

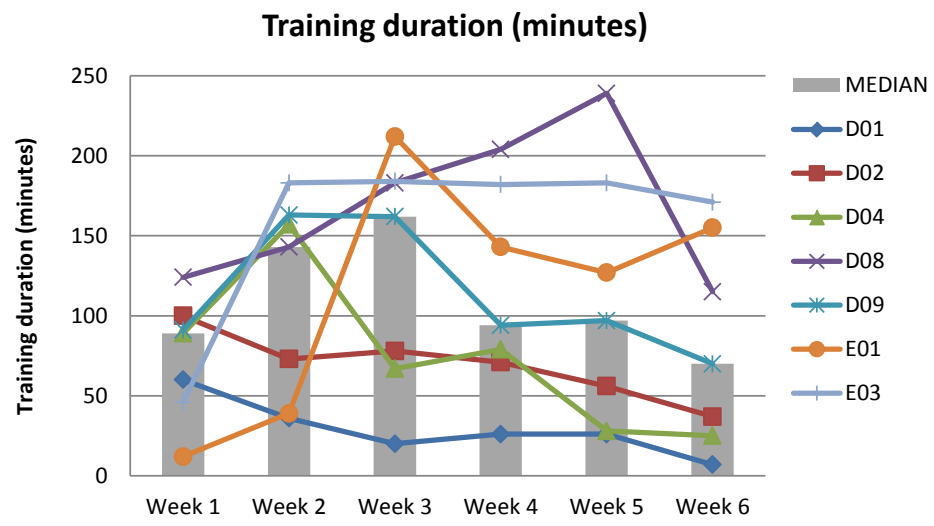

Figure 7.2 Individual (colored lines) and group median (grey bars) weekly training duration

The median score on the SUS was 73\% (IQR 60\% - 83\%). On individual level, four participants rated usability over $70 \%$ and three participants between 50 and $70 \%$. Overall, the participants enjoyed the six weeks of training, as reflected in the overall median score on the IMI of 4.4 points (IQR $3.9-6.0$ points). Table 7.2 shows individual participant results and group medians on all outcomes.

\section{Arm and hand function tests}

On group level, the Wilcoxon signed rank test showed a significant improvement after training for the FM total, FM proximal part, ARAT, and MAL AOU (Table 7.3). The FM showed a median improvement of 4.0 points $(P=0.034)$ for the total scale, and median improvement of 3.0 points $(P=0.027)$ for the proximal part only. The ARAT improved with median 2.0 points $(P=0.045)$ over training, and the MAL AOU with median 0.2 points $(P=0.046)$.

Examination of individual scores (Table 7.2) shows quite substantial improvements for one participant (E01), grossly exceeding the minimal clinically important difference (MID) for FM and $\mathrm{ARAT}^{26}{ }^{26}$ constituting changes greater than $10 \%$ of the total score. Participant E03 approaches MCID for FM with six points improvement. In addition, two participants (D09 and E03) exceeded MCID for the MAL QOM, of which E03 also exceeded MCID on MAL AOU, ${ }^{27}$ and participant E01 approaches MCID for both MAL AOU and QOM. 
Table 7.2 Individual participant results and group medians on all outcome measures

\begin{tabular}{|c|c|c|c|c|c|c|c|c|}
\hline $\begin{array}{l}\text { Partici- } \\
\text { pant }\end{array}$ & $\begin{array}{c}\text { Average } \\
\text { weekly } \\
\text { training } \\
\text { duration } \\
\text { (minutes) }\end{array}$ & IMI (1-7) & SUS (\%) & $\begin{array}{l}\text { FM change } \\
(\max =66)\end{array}$ & $\begin{array}{c}\text { ARAT } \\
\text { change } \\
(\max =57)\end{array}$ & $\begin{array}{c}\text { MAL AOU } \\
\text { change } \\
(0-5)\end{array}$ & $\begin{array}{c}\text { MAL QOM } \\
\text { change } \\
(0-5)\end{array}$ & $\begin{array}{c}\text { SIS change } \\
(\%)\end{array}$ \\
\hline D01 & 29 & 4.0 & 60 & -1 & 2 & -0.04 & -0.26 & 1.8 \\
\hline D02 & 69 & 3.4 & 73 & 1 & -1 & 0.00 & 0.00 & -0.6 \\
\hline D04 & 74 & 3.9 & 68 & 3 & 2 & 0.28 & -0.18 & -3.7 \\
\hline D08 & 168 & 6.0 & 83 & 4 & 0 & 0.05 & 0.05 & 10.6 \\
\hline D09 & 113 & 6.7 & 95 & 4 & 3 & 0.18 & 0.67 & 3.2 \\
\hline E01 & 115 & 4.4 & 58 & 15 & 13 & 0.44 & 0.44 & 11.0 \\
\hline E03 & 158 & 4.7 & 73 & 6 & 3 & 0.68 & 0.56 & 9.3 \\
\hline $\begin{array}{l}\text { Median } \\
\text { (IQR) }\end{array}$ & $\begin{array}{c}113 \\
(69-158)\end{array}$ & $\begin{array}{c}4.4 \\
(3.9-6.0)\end{array}$ & $\begin{array}{c}73 \\
(60-83)\end{array}$ & $\begin{array}{c}4 \\
(1-6)\end{array}$ & $\begin{array}{c}2 \\
(0-3)\end{array}$ & $\begin{array}{c}0.2 \\
(0.0-0.4)\end{array}$ & $\begin{array}{c}0.1 \\
(-0.2-0.6)\end{array}$ & $\begin{array}{c}3.2 \\
(-0.6-10.6)\end{array}$ \\
\hline
\end{tabular}

Abbreviations: IMI: Intrinsic Motivation Inventory, SUS: System Usability Scale, FM: Fugl-Meyer assessment, ARAT: Action Research Arm Test, MAL AOU: Motor Activity Log Amount of Use, MAL QOM: Motor Activity Log Quality of Movement, SIS: Stroke Impact Scale, IQR: Interquartile range

Table 7.3 Arm and hand function tests (median (IQR))

\begin{tabular}{lccc}
\hline Outcome & Pre measurement & Post measurement & P-value \\
\hline FM & $37(30-45)$ & $41(33-49)$ & $\mathbf{0 . 0 3 4}$ \\
FM Proximal & $21(21-31)$ & $25.0(23-32)$ & $\mathbf{0 . 0 2 7}$ \\
FM Distal & $14(9-17)$ & $16(10-18)$ & 0.131 \\
ARAT & $26(21-28)$ & $28(23-31)$ & $\mathbf{0 . 0 4 5}$ \\
MAL AOU & $0.8(0.4-1.5)$ & $0.9(0.8-1.4)$ & $\mathbf{0 . 0 4 6}$ \\
MAL QOM & $0.8(0.4-1.2)$ & $0.8(0.7-1.3)$ & 0.249 \\
SIS & $61.4(50.6-68.6)$ & $66.2(50.8-72.1)$ & 0.128 \\
\hline
\end{tabular}

Abbreviations: FM: Fugl-Meyer assessment, ARAT: Action Research Arm Test, MAL AOU: Motor Activity Log Amount of Use, MAL QOM: Motor Activity Log Quality of Movement, SIS: Stroke Impact Scale.

\section{Discussion}

A second generation technology-supported arm and hand training system was evaluated in patients with chronic stroke in their own home. The present findings show that training at home using the training system was feasible, since patients accepted the training well (median SUS $=73 \%$ ) and were motivated (median IMI $=4.4$ ). This was reflected in a median weekly training duration of 113 minutes (i.e., approximately 15 minutes per day). Participants showed improvements in arm and hand function, dexterity and self-perceived amount of arm and hand use in daily life. 
The motivation outcomes of the current study indicate that patients perceived the training as motivating, to a similar extent as interventions applying rehabilitation technology in a clinical setting. ${ }^{28,}{ }^{29}$ With a median SUS score of $73 \%$ the training system was rated as promising. This might be related to a large variation of games available in the current study, which was much appreciated by the participants. Although participants positively valued the training system, several usability issues were identified and should be considered when implementing further design adaptations.

In particular, some games caused errors after leaving the pause screen resulting in incorrect saving of the data, controlling one game was not clear for some participants, and another game had limited fluent game control in poor day light. The game utilized the position of a marker on the orthosis to determine hand position in space, however poor day light impacted on capturing this position accurately. Although these issues were not major, if repeated or cumulative, they are likely to result in frustration and might influence the motivation and attitude towards use of the system, which can negatively affect the adherence to training over time. ${ }^{30}$

In the current study, participants were able to make their own decisions about their training schedule, without direct, real-time supervision of a therapist. The rationale for this was to remove the training constraints and increase therapy availability. Compared to previous telerehabilitation studies in which training sessions are often scheduled beforehand and with direct supervision, ${ }^{13,31}$ the achieved training duration of median 113 minutes per week was substantial, suggesting that stroke patients do have the incentive to train at home and were able to use the system. This adherence falls within the range reported in other recent studies into technology-supported home-based self-administered upper limb therapy programs after stroke. ${ }^{30,32,33}$ On the other hand, most participants did not reach the advised training duration, which is also comparable to these previous home-based studies. ${ }^{15,30,32,33}$

One of the major assumptions concerning tele-rehabilitation using technologies is that when patients accept the technology and clinically benefit from it, they will actually use such a system when provided. However in practice, several factors, such as low motivation, fatigue and musculoskeletal issues can result in limited adherence, ${ }^{34}$ while training dose is an important factor in rehabilitation outcome. ${ }^{35}$ When considering individual results, participants who had a rather high amount of training per week (>100 minutes), showed substantial improvements on arm and hand function. This suggests that actual adherence during self-administered practice is a highly relevant outcome. Moreover, stimulation of adherence should receive wide attention when designing and implementing home-based training interventions. 
Several strategies can be considered for stimulation of adherence. Research has shown that regular patient-therapist contact during treatment has a motivational effect and can increase therapy adherence. ${ }^{36}$ In our study, additional motivational strategies were implemented in a subset of our games, ${ }^{37}$ to increase participants' independent training time at home. This comprised more direct feedback about training duration, such as showing motivational messages about the duration after completion of each game, or continuously showing a timer during game play. We incorporated approaches from the field of psychology and education theory to further overcome this barrier, such as setting the correct balance between supporting and challenging, to maximize adherence to therapy. ${ }^{37}$ However, our adaptive game-difficulty setting was not available in all games. In addition, patients' selfdiscipline might also play a role in this kind of unsupervised home training. So in future, it is also valuable to look closer at other characteristics such as patients' attitudes, personality traits, coping skills and commitments in daily life ${ }^{30,32}$ to enable understanding of the most suitable patients for this kind of self-administered training.

The extent of improvement in motor function of the arm in the present study corresponded with those found in other robot-aided studies in chronic stroke in a clinical setting, ${ }^{4-7}$ and with therapy programs for the upper limb performed at home. ${ }^{13}$ Perceived use of the affected arm in daily life as assessed by the MAL did significantly change on group level after six weeks training, which was also reflected in an improved dexterity capacity as measured by ARAT. Three of the games contained functional movements: integration of reaching, grasping and transportation simultaneously, and the inclusion of specific grasps, such as cylinder grasp, lateral grasp and palmar prehension grasp, to represent handling of different objects. This might have played a role in the improvements on activity level in the current study, since task-specificity is an important factor in restoration of arm and hand function after stroke. ${ }^{9,38}$ However, these improvements are still modest on group level and not clinically relevant in terms of functional improvements. On the other hand, games with more complex gestures were only made available to patients after some progress was made in simpler games which could impact on the extent of these modest improvements. When these aspects are incorporated more prominently and in more games, exercises become even more functional and task-specific, which is expected to further enhance the clinical impact.

This study was performed with chronic stroke patients, limiting bias from spontaneous recovery and simultaneous other treatments. Home-based training could be considered at an earlier stage, where larger treatment effects would be expected. Further, only data of seven chronic stroke patients with mainly moderate stroke are available. Findings of the present study can only be partly generalized to other stroke survivors because of the small number of participants in this study. Future research should consider implementing a large 
randomized controlled trial, with sufficient statistical power to compare the effects with a control group.

\section{CONCLUSION}

The positive results for motivation, usability and actual training duration in this study indicate that home-based technology-supported arm and hand training is a feasible tool to enable self-administered practice at home. The improved arm and hand function and increased performance on activity level (both actual as well as perceived) indicates that self-administered home-based training can have a clinical value. Such an application has the potential to allow a higher dose of treatment than would be possible when depending on therapist availability in a conventional setting, if adherence can be stimulated further. Future research regarding telerehabilitation should therefore pay attention to adherence (stimulation) and the functional nature of exercises. Furthermore, identification of factors associated with better treatment outcomes (e.g., time post stroke, stroke severity and personal characteristics) is needed in order to understand who would benefit most from this technology-supported training at home.

\section{REFERENCES}

1. Hendricks HT, van Limbeek J, Geurts AC and Zwarts MJ. Motor recovery after stroke: a systematic review of the literature. Arch Phys Med Rehabil. 2002; 83: 1629-37.

2. Taub E, Uswatte G, Mark V and Morris D. The learned nonuse phenomenon: implications for rehabilitation. Eura Medicophys. 2006; 42: 241-55.

3. Taub E and Morris DM. Constraint-induced movement therapy to enhance recovery after stroke. Current atherosclerosis reports. 2001; 3: 279-86.

4. Mehrholz J, Pohl M, Platz T, Kugler J and Elsner B. Electromechanical and robot-assisted arm training for improving activities of daily living, arm function, and arm muscle strength after stroke. Cochrane Database Syst Rev. 2015; 11: CD006876.

5. Maciejasz P, Eschweiler J, Gerlach-Hahn K, Jansen-Troy A and Leonhardt S. A survey on robotic devices for upper limb rehabilitation. Journal of Neuroengineering and Rehabilitation. 2014; 11: 3.

6. Prange GB, Jannink MJ, Groothuis-Oudshoorn CG, Hermens HJ and ljzerman MJ. Systematic review of the effect of robot-aided therapy on recovery of the hemiparetic arm after stroke. J Rehabil Res Dev. 2006; 43: 171-84.

7. Basteris A, Nijenhuis SM, Stienen AH, Buurke JH, Prange GB and Amirabdollahian F. Training modalities in robot-mediated upper limb rehabilitation in stroke: a framework for classification based on a systematic review. J Neuroeng Rehabil. 2014; 11: 111.

8. Wagenaar RC and Meijer OG. Effects of stroke rehabilitation: a critical review of the literature Journal of Rehabilitation Sciences. 1991; 4: 61-73.

9. Timmermans AA, Seelen HA, Willmann RD and Kingma H. Technology-assisted training of arm-hand skills in stroke: concepts on reacquisition of motor control and therapist guidelines for rehabilitation technology design. J Neuroeng Rehabil. 2009; 6: 1.

10. Balasubramanian S, Klein J and Burdet E. Robot-assisted rehabilitation of hand function. Curr Opin Neurol. 2010; 23: 661-70.

11. Brennan DM, Mawson S and Brownsell S. Telerehabilitation: enabling the remote 
delivery of healthcare, rehabilitation, and self management. Stud Health Technol Inform. 2009; 145: 231-48.

12. Howard G and Goff DC. Population shifts and the future of stroke: forecasts of the future burden of stroke. Ann N Y Acad Sci. 2012; 1268: 14-20.

13. Johansson T and Wild C. Telerehabilitation in stroke care--a systematic review. J Telemed Telecare. 2011; 17: 1-6.

14. Piron L, Turolla A, Tonin P, Piccione F, Lain L and Dam M. Satisfaction with care in post-stroke patients undergoing a telerehabilitation programme at home. Journal of telemedicine and telecare. 2008; 14: 257-60.

15. Nijenhuis SM, Prange GB, Amirabdollahian F, et al. Feasibility study into self-administered training at home using an arm and hand device with motivational gaming environment in chronic stroke. Journal of Neuroengineering and Rehabilitation. 2015; 12: 89.

16. Amirabdollahian F, Ates S, Basteris A, et al. Design, development and deployment of a hand/wrist exoskeleton for home-based rehabilitation after stroke - SCRIPT project. Robotica. 2014; 32: 1331-46.

17. Ates $S$, Leon B, Basteris A, et al. Technical evaluation of and clinical experiences with the SCRIPT passive wrist and hand orthosis. 7th International Conference on Human System Interactions (HSI). 7th ed.: IEEE, 2014, p. 188-93.

18. Leon B, Basteris A, Infarinato $F$, et al. Grasps recognition and evaluation of stroke patients for supporting rehabilitation therapy. Biomed Res Int. 2014; 2014: 318016.

19. McAuley E, Duncan T and Tammen VV. Psychometric properties of the Intrinsic Motivation Inventory in a competitive sport setting: a confirmatory factor analysis. Res Q Exerc Sport. 1989; 60: 48-58.

20. Brooke J. SUS: A Quick and Dirty Usability Scale. In: Jordan PW, Thomas B, Weerdmeester BA and McClelland IL, (eds.). Usability Evaluation in Industry. London: Taylor \& Francis., 1996.

21. Bangor A, Kortum P and Miller J. Determining what individual SUS scores mean: Adding an adjective rating scale. Journal of usability studies. 2009; 4: 114-23.

22. Fugl-Meyer AR, Jaasko L, Leyman I, Olsson S and Steglind S. The post-stroke hemiplegic patient. 1. A method for evaluation of physical performance. Scand J Rehabil Med. 1975; 7: 13-31.

23. Lyle RC. A performance test for assessment of upper limb function in physical rehabilitation treatment and research. Int J Rehabil Res. 1981; 4: 483-92.

24. van der Lee $\mathrm{JH}$, Beckerman $\mathrm{H}$, Knol DL, de Vet $\mathrm{HC}$ and Bouter LM. Clinimetric properties of the motor activity log for the assessment of arm use in hemiparetic patients. Stroke. 2004; 35: 1410-4.

25. Duncan PW, Wallace D, Lai SM, Johnson D, Embretson S and Laster LJ. The stroke impact scale version 2.0. Evaluation of reliability, validity, and sensitivity to change. Stroke. 1999; 30: 2131-40.

26. van der Lee JH, Beckerman $H$, Lankhorst GJ and Bouter LM. The responsiveness of the Action Research Arm test and the Fugl-Meyer Assessment scale in chronic stroke patients. J Rehabil Med. 2001; 33: 110-3.

27. van der Lee JH, Wagenaar RC, Lankhorst GJ, Vogelaar TW, Deville WL and Bouter LM. Forced use of the upper extremity in chronic stroke patients: results from a single-blind randomized clinical trial. Stroke. 1999; 30: 2369-75.

28. Prange $\mathrm{GB}$, Kottink Al, Buurke $\mathrm{JH}$, et al. The effect of arm support combined with rehabilitation games on upper-extremity function in subacute stroke: a randomized controlled trial. Neurorehabil Neural Repair. 2015; 29: 174-82.

29. Colombo R, Pisano F, Mazzone A, et al. Design strategies to improve patient motivation during robot-aided rehabilitation. J Neuroeng Rehabil. 2007; 4: 3. 
30. Standen PJ, Threapleton K, Connell L, et al. Patients' Use of a Home-Based Virtual Reality System to Provide Rehabilitation of the Upper Limb Following Stroke. Phys Ther. 2014; 11: 11.

31. Laver KE, Schoene D, Crotty M, George S, Lannin NA and Sherrington C. Telerehabilitation services for stroke. Cochrane Database Syst Rev. 2013; 12: CD010255.

32. Wolf SL, Sahu K, Bay RC, et al. The HAAPI (Home Arm Assistance Progression Initiative) Trial: A Novel Robotics Delivery Approach in Stroke Rehabilitation. Neurorehabil Neural Repair. 2015; 29: 958-68.

33. Donoso Brown EV, McCoy SW, Fechko AS, Price R, Gilbertson T and Moritz CT. Preliminary investigation of an electromyography-controlled video game as a home program for persons in the chronic phase of stroke recovery. Arch Phys Med Rehabil. 2014; 95: 14619.

34. Jurkiewicz MT, Marzolini S and Oh P. Adherence to a home-based exercise program for individuals after stroke. Topics in Stroke Rehabilitation. 2011; 18: 277-84.

35. Kwakkel G. Intensity of practice after stroke: More is better. Schweizer Archiv für Neurologie und Psychiatrie. 2009; 7: 295-8.

36. Jette $A M$, Rooks $D$, Lachman $M$, et al. Home-based resistance training: predictors of participation and adherence. Gerontologist. 1998; 38: 412-21.

37. Basteris A, Nijenhuis SM, Buurke JH, Prange GB and Amirabdollahian F. Lag-lead based assessment and adaptation of exercise speed for stroke survivors. Robotics and Autonomous Systems. 2015; 73: 144-54.

38. Kitago T and Krakauer JW. Chapter 8 - Motor learning principles for neurorehabilitation. In: Michael PB and David CG, (eds.). Handb Clin Neurol. Elsevier, 2013, p. 93-103. 
Feasibility of post-stroke arm and hand training at home 2 



\section{Chapter 8}

General discussion 
Many physical therapy interventions in stroke rehabilitation are currently available. ${ }^{1-3}$ However, it is not always clear which type of intervention is most successful in promoting upper extremity motor recovery. The use of technology-supported devices, such as rehabilitation robotics, has been shown to be effective for rehabilitation of the arm and hand after stroke. ${ }^{4,5}$ The literature review in chapter 2 reveals that most of the studies so far on upper extremity robot rehabilitation focus on training of the proximal arm in chronic stroke patients, although integration of distal with proximal arm training is thought to be most effective to enhance functional gains. ${ }^{6}$ Features stressing active contribution by the patient revealed most beneficial for improvements in arm function (chapter 2). Therefore, active contribution of the patient during training, involving functional movements of both the proximal and distal arm and hand, was incorporated within the SCRIPT training study. This was succeeded by providing a passive-actuated orthosis equipped with sensors. This orthosis was worn on the forearm and hand, requiring active initiation and execution of arm and hand movements by the patient to control functional exercise games for training. In the subsequent studies described in this thesis we evaluated this newly developed technologysupported prototype, used for training of the arm and hand after stroke. Compared to many other technology-supported devices currently available, the SCRIPT training system had the advantage to easily transport it to the patients' homes, to encourage independent practice without direct supervision of a therapist, and as such provoke a high training dose. With this thesis, we aimed to contribute to the knowledge concerning self-administered technology-supported, functional and motivational training of the arm and hand at home in the chronic phase after stroke, in terms of feasibility and potential clinical effects. As part of this, we were interested to assess whether patients can work with the training system independently, do they experience it as usable? If patients have the opportunity to use a technology-supported training system at home, do they actually use it? Other relevant questions concerned the motivation to train and to evaluate the (direct) effects of using the training system. In this chapter we will combine and discuss the results of the subsequent studies in this thesis, which gradually provided more insight in the potential of technologysupported training of the arm and hand after stroke at home. In addition, valuable lessons were learned during the two consecutive evaluation phases of the SCRIPT training system during the iterative design process, which gave rise to recommendations that could be considered for future research and developments in the field of technology-supported arm and hand training at home after stroke.

\section{ITERATIVE DEVELOPMENT OF THE PROTOTYPE TRAINING SYSTEM}

According to the framework presented by Jansen-Kosterink et al. 2016, a staged approach for the development and evaluation of telemedicine services is recommended. ${ }^{7}$ The SCRIPT project followed this approach twice, in a slightly adapted and short form. An application such as the SCRIPT device, which covers rehabilitation technology with a telemedicine 
component, is supposed to have a direct training effect. Therefore, a logical step would be to evaluate the prototype already in an early stage in the form of a pilot longitudinal study as a means to gain insight into its potential effect. Therefore, our development and evaluation procedure followed several phases in an iterative cycle design process (Figure 8.1), but mainly focused on the first two stages of the staged approach framework for evaluation of eHealth presented by Jansen-Kosterink et al. 2016. ${ }^{7}$

As part of this approach, several usability aspects of the prototype were addressed before evaluation of the complete system in the patients' homes. A first prototype training system was developed based on knowledge in literature and stroke patients' preferences and experiences with technology. ${ }^{8}$ Multiple prototypes of the passive hand and wrist orthosis have been evaluated by patients and healthcare professionals before being ready for independent training at home. This resulted in an orthosis providing assistance in wrist and finger extension with natural range of motion of the wrist and hand, but also safe enough to use independently at home. ${ }^{9}$ Before using it as a training device at home, this orthosis was evaluated in a cross-sectional study in the lab (chapter $\mathbf{3}$ ) to evaluate its working mechanism and to identify which design aspects and usability issues needed further attention. During this cross-sectional measurement, the direct effects of the orthosis on arm and hand movements were measured. The use of the orthosis had a positive direct influence on maximum hand opening. Although predictable, confirmation of this positive outcome supports the notion that the orthosis enables patients with hand opening and closing difficulties due to stroke to facilitate their hand movements during training, and allows patients to grasp large objects. On the other hand, the use of the orthosis had a negative direct influence on the trunk displacement (increased) and elbow range of motion (decreased), probably due to the weight of the orthosis on the arm causing larger compensatory movements. The use of an additional device supporting the arm against gravity was therefore recommended when using this orthosis as a training device at home, to counteract potential compensatory trunk movements. In previous research, the use of such a device has been shown to have positive effects on reaching work area. ${ }^{10,11}$ Weight support of the hemiparetic arm compensates for abnormal muscle coactivation resulting in synergistic joint torque coupling of shoulder abduction and elbow flexion. ${ }^{10,12}$ Consequently, the use of a device supporting the arm against gravity was also implemented during the feasibility studies in chapter 4 and chapter 7 , which contributes to functional training of both proximal and distal arm and hand movements.

The feasibility study in chapter 4 taught us first of all that the training system was a feasible tool to enable self-administered practice. Usability of the system was rated sufficient at group level (mean 69\%, median 68\%), although some usability issues were identified during the first evaluation that needed further attention. 
Chapter 8

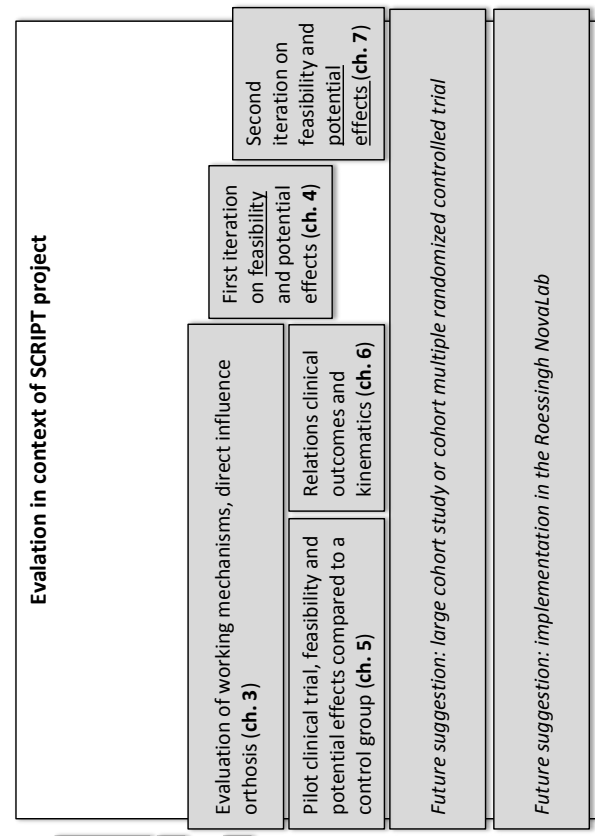

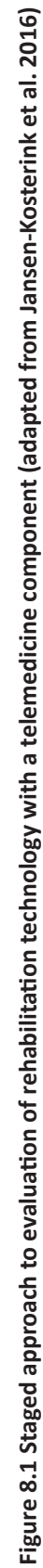

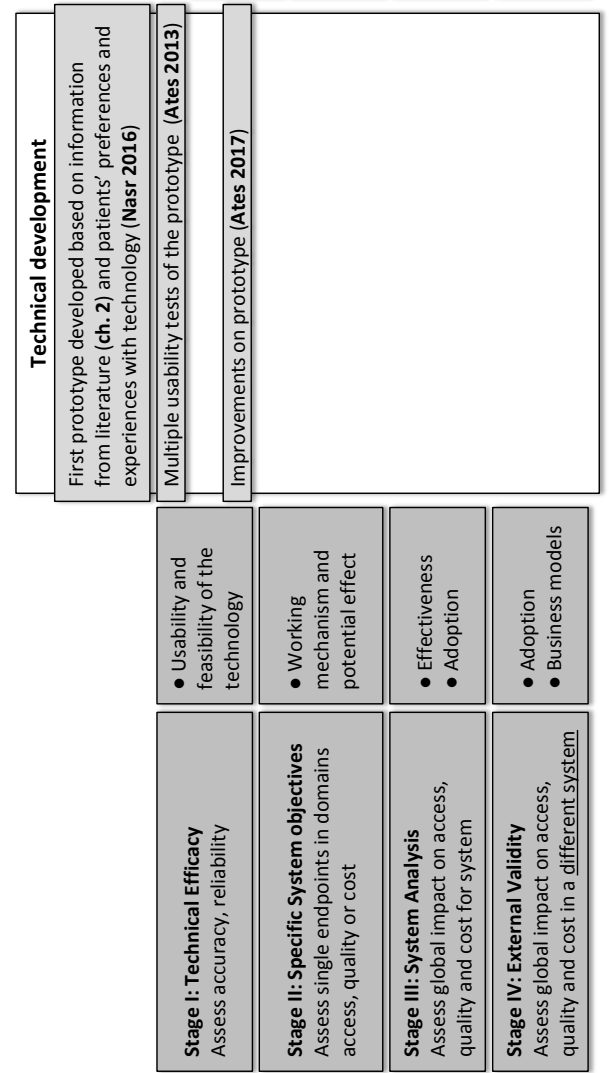


First, some patients mentioned technical problems with the system, such as an elastic cord used for supporting finger extension that occasionally broke down after frequent use, errors within the games resulting in a sudden black screen, or a sensor which broke down causing a game to become unresponsive. ${ }^{13}$ Second, some patients had problems to successfully complete a game due to problems with game control: the sensors at the leaf springs for finger position and hand plate to track arm movements turned out to be limited in accuracy, which affected interaction with the software environment. ${ }^{13}$ In addition, the elastic cords were not strong enough to provide sufficient extension torque for the more severely impaired patients. And last, since the hand orthosis was perceived as too bulky and felt heavy, this required the need of an additional arm support for compensation of the arm against gravity, which is in line with the direct influence of the orthosis as found in chapter 3 . This contributed to a large donning and doffing time. In addition to an extensive calibration procedure required before actually starting a game, this required quite some preparation steps and effort before patients could actually start a training session. These usability issues caused frustration in some of the patients, and were serious reasons for not using the system as frequently as intended. Many of these issues were taken into account during the development of the second iteration SCRIPT training device ${ }^{13}$, resulting in indeed, better usability outcomes (chapter 7 , mean $=73 \%$, median $=73 \%$ ) compared to the first iteration feasibility study (chapter 4 , mean $=69 \%$, median $=68 \%$ ), as measured with the System Usability Scale (SUS). The SUS scale determines that scores in the 70s and 80 s are promising and have potential for acceptance in the field. Interventions with usability scores below $70 \%$ should increase the accuracy and need continued improvement. ${ }^{14}$ Furthermore, results of chapter 4 and 7 revealed that patients do have the incentive to train at home, with on average 105 minutes per week (median = 100 minutes) during the first evaluation, and 104 minutes per week (median = 113 minutes) during the second iteration. Apparently, participants were able to complete self-administered practice at home independently, despite the above mentioned usability issues. In addition, motivation was positive and comparable during both evaluations, with a mean of 5.2 (median $=5.3$ ) out of seven during the first evaluation and mean of 4.7 (median $=4.4$ ) during the second evaluation, which are both above the midpoint of the Intrinsic Motivation Inventory. For future research, it is suggested to take abovementioned usability aspects into account, by providing a robust, reliable and safe training device. It is recommended to provide an easy to apply system for training, which is easy to use independently. Subsequently, such a device can be evaluated further in a large cohort study or cohort multiple randomized controlled trial as the next stage (stage 3 ) in evaluation of rehabilitation technology with a telemedicine component (Figure 8.1). 


\section{THE IMPORTANCE OF FUNCTIONAL TRAINING}

On group level, upper extremity function improved significantly after the six weeks of training. During both the first and second evaluation, scores on the Fugl-Meyer assessment increased with four points over training. No generalization is found to improvements in upper limb activity capacity during the first evaluation (chapter 4 ), whereas the second evaluation (chapter 7) did show some moderate improvements in activity capacity, specifically dexterity in this case, as represented by an average improvement of two points (median improvement = 3.1) on the Action Research Arm Test. Our results correspond with those found in other robot-aided studies in chronic stroke in a clinical setting ${ }^{5,15,16}$ (chapter 2) and with therapy programs for the upper extremity performed at home. ${ }^{17-19}$ The improvements found in our studies involved particularly proximal arm movements, especially during the first evaluation phase. Apparently, the exercises appeared not diverse or functional enough yet to reveal functional gains. Primarily during the first evaluation, the three games available for practice mostly required movements of the arm, wrist and hand in sequence. In addition, the hand gestures were rather course and did not contain specific grasps representing the handling of various objects. This may have played a role in the marginal effects on activity level, because proximal and distal arm and hand movements were not integrated optimally yet. The expectation was that when such aspects are integrated more specifically, exercises become even more functional and task-specific, which are important factors in achieving functional benefits on arm and hand function after stroke. ${ }^{6,20,21}$

These aspects have been considered in designing six additional games for the second evaluation in chapter $\mathbf{7}$. Here, more versatile grasping gestures were incorporated, and translational movements of the arm were integrated with wrist and hand movements in parallel instead of sequentially, to emphasize functional, challenging and varying movements. This resulted in improvements on both upper extremity function, activity capacity and selfperceived activity performance in daily life after six weeks of training (chapter $\mathbf{7}$ ), whereas the improvements on upper extremity function were not transferred to improvements on activity level during the first evaluation phase (chapter 4).

Chapter 6 further emphasized the importance of providing functional movements for training, in which relationships between an extensive set of objective movement execution kinematics of the upper extremity and clinical outcome measures in chronic stroke patients were measured. Elbow excursion and grip strength together explained the largest amount of variance in the Fugl-Meyer assessment and Action Research Arm Test. This is comparable to previous research in stroke patients showing relations between clinical outcome measures and kinematic outcomes involving reaching and hand function. ${ }^{22}$ This implies that addressing both reaching and hand function are indicated as valuable targets for (technology-supported) treatment applications to stimulate functional improvements 
after stroke. Therefore, for future research we suggest to provide challenging, task-specific and a high variety of exercises, since this is expected to have a positive influence on clinical outcomes, and even might work motivational as well.

\section{THE IMPORTANCE OF A HIGH TRAINING DOSE}

If patients are able to make their own decisions about their training schedule and are not dependent on therapist availability, this will remove training constraints. Therefore, it is assumed that training at home is a good solution to encourage independent training and consequently enhance a high training dose. A high training dose, so the effective training duration, has been shown to be an important factor for obtaining functional gains. ${ }^{23}$ Although the optimal training dose is not known yet, 16 hours of additional training is recommended to achieve clinically relevant functional improvements. ${ }^{24}$ This corresponds closely with our advised dose of 30 minutes per day for 6 days per week, which is 18 hours total during the six weeks. Results of chapter 4 and 7 revealed that patients do have the incentive to train at home, but with a large amount of variation in effective use of the system between individuals (range $=13-284$ minutes per week). The average use was 105 minutes (median $=100$ ) per week during the first evaluation (chapter 4 ), and 104 minutes per week (median $=113$ ) during the second iteration (chapter 7 ). When comparing these results with other studies into home-based self-administered upper extremity programs after stroke, the use of the training system equals or even exceeds the adherence in those studies. ${ }^{18,}$ 25-28 Differently than what was assumed, the training duration is not tremendously higher compared to technology-supported interventions performed in the clinic, ${ }^{4,15}$ although there is a considerable variability in how interventions are delivered with respect to intensity, frequency and duration. ${ }^{4,15}$ The training sessions performed in the clinic are often scheduled beforehand and involve direct supervision by a therapist. It is known that human support from a healthcare provider can influence the adherence to training..$^{29}$ Our studies involved limited support from a healthcare professional, with only home visits once per week, and training was mainly performed independently by the patient.

Despite that patients do have the incentive to train at home independently, individual training at home resulted in a lower adherence to training than the advised training dose. Reasons for patients not making the advised training dose could be related to their reintegration into daily life. A large part of the patients were moderately to mildly impaired, continuing their activities in daily life, although slightly limited. This means that many of the patients were reintegrated to work, or had to take care of their family. Some patients mentioned this was quite tiring and the time remaining to dedicate to the exercise training was limited. Therefore, when designing more motivational systems to enhance independent training dose, practical issues such as regular daily activities and obligations (such as work, family life, etc.), should not be ignored either. 
The importance of having a high training duration is emphasized in chapter $\mathbf{5}$, in which correlation analyses were performed for training duration and changes in clinical outcomes, next to a comparison of the SCRIPT system with a control group performing conventional exercises from an exercise book. Individual patient outcomes indicated that training duration in itself seems a major contributor to arm and hand function improvements. No differences in clinical outcomes over training were found between both groups. However, we did find a slightly higher training duration for the control group. This was surprising since motivational exercise games and feedback were expected to enhance engagement to training, resulting in a higher motivation and a higher training dose for the SCRIPT training group, ${ }^{5,30}$ since maintaining strong participant motivation can influence the adherence to training. ${ }^{29}$ However, training duration in chapter 5 was recorded in a personal diary for both groups. The participants in the experimental group knew that their training duration was also automatically stored within the system, probably resulting in lower overestimation of their training time compared to the control group. The training duration reported by the control group could not be verified objectively and is likely overestimated, since it is known that many people will overestimate self-reported physical activity. ${ }^{31}$ Other reasons for finding a lower training duration for the experimental group than expected could be related to the usability issues mentioned before, or the diversity of the training. In the study presented in chapter 5 , three games were available for the experimental group, versus maximally 34 exercises for the control group. This may have played a role in the higher training duration of the control group. A large variety of attractive functional exercises is important to prevent boredom and keep patients motivated to continue training. The weekly training duration remained quite stable during the six weeks of training for both the control group and the group using the SCRIPT training system. Also perceived motivation was equally positive for both groups. One may argue if participants were really motivated to complete a training session, or just pleasing the researchers, with a risk of participation bias. It is not known whether patients would continue training beyond the six weeks of training if they had the chance. More attention should be provided to determine effective strategies for ensuring high motivation and adherence to training, ${ }^{32}$ since a high training dose is an important factor for obtaining functional gains. ${ }^{23}$ The question is how we can achieve that.

\section{SUgGeSTIONS TO ENHANCE TRAINING ADHERENCE AND MOTIVATION}

To enhance motivation to training, it is important to offer a high variety of challenging games, with enough variation in gestures to control the games. Monotonous exercises have been shown to provide worse retention and generalizability of a skill compared with variable exercises for training. ${ }^{33}$ The SCRIPT system was extended with six additional games in chapter 7 in comparison to the first study (chapter 4). For further development of the system, the number of available games for practice could be extended even more. 
Within the SCRIPT training study, all participants exercised alone during the training sessions. This might be a risk considering motivation, since patients had no interaction with other people during training. A promising method to increase motivation is by means of interpersonal rehabilitation games, such as competition or cooperation with other people. A stroke patient playing competitive games against unimpaired friends or relatives has high potential for motor rehabilitation, since this has been shown to increase both motivation and intensity. ${ }^{34}$ In general, the involvement of caregivers or peers in exercise training at home might be an efficient method to enhance the motivation and intensity of training, although the evidence is limited yet. ${ }^{35,36}$ At least the patients who participated in the training studies appreciated the weekly home visits from the healthcare professional, indicating that interaction with other people is motivational. This might have had a positive influence on the training duration, since home visits might improve adherence to therapy. ${ }^{37}$ In future, more and more eHealth applications will be implemented in healthcare, but it is preferred to maintain some kind of personal contact with healthcare providers. ${ }^{38}$

Another factor which might result in better motivation to training is the provision of meaningful feedback. ${ }^{39}$ Providing extrinsic feedback has resulted in implicit motor learning and improving upper extremity motor recovery in stroke patients. ${ }^{40}$ Feedback can be provided either during or following the task performance, and might involve aspects such as sounds, scores for task success or feedback about completed training time. The optimal type and schedule of feedback delivery might need further research, ${ }^{40}$ and probably also depends on different personality characteristics of individual patients. For the SCRIPT training system, additional motivational strategies might be implemented to increase participants' effective training time during self-administered training at home. Future studies might consider approaches from the field of psychology to further explore this potential and to explore for what kind of patients this kind of intervention is most appropriate.

\section{TAILORED INTERVENTIONS}

Recommendations specific to dose and type of intervention, target users (regarding severity of impairment, time post injury or other factors) and expected outcomes are often not specifically described in guidelines. ${ }^{41,42}$ Very recently, research has proposed clinical tools for choosing the most appropriate intervention, taking into consideration time post stroke and stroke severity, such as the presence of hand movement and the presence of spasticity. ${ }^{2}$ ${ }^{41}$ This is a first step in choosing an appropriate intervention for a specific patient. In health research, often a 'one size fits all' approach is applied. However, what is generally considered the best care for a group a patients is not by definition the best care for an individual patient. Personalized care is preferred, to adjust the therapy program to the specific characteristics and (most likely continuously changing) needs of the patient. However, it is still challenging to tailor training strategies to the needs of the individual patient. ${ }^{2}$ 
Within the SCRIPT project we attempted to provide a more personalized training program, by providing a custom-built orthosis for training that physically interacted with the arm and hand, and providing a variety of exercise games for training. These games were developed based on patients' preferences and suggestions. ${ }^{8}$ The exercise games contained adaptive features since the type and speed of exercises were based on the patient's specific capability and training progress and could change over time by using a decision support system. ${ }^{43}$, ${ }^{44}$ This was supported and verified by weekly home visits of a healthcare professional to provide the best targeted training for the specific patient. Besides, patients were able to choose their own preferred games for practice from the selected ones, although the selection was limited to three (chapter 4 ) or nine (chapter 7 ) games in total.

However, for what kind of patients (in terms of stroke severity and time post stroke) the complete training system was most suitable, was not clear yet. Therefore, during the first evaluation phase of the SCRIPT training system at home (chapter 4), we included a wide range of patients for training. This evaluation showed us a large amount of variation in effective use of the system (range $=13-284$ minutes per week) between and within individuals, but also on usability (range $=38-100 \%$ ) and motivation (range $=3.4-6.7$ points) between individuals. Considering clinical outcomes, some participants did achieve clinically relevant gains in arm and hand function, while others did not. From this sample of 21 patients, it appears that mildly to moderately impaired patients benefitted most from the intervention, which is consistent with previous upper extremity robotic exercise training. ${ }^{45}$ In addition, severely impaired patients commented most about the difficulty of training, and also showed least improvements. This emphasizes the need for other or better-tailored therapy strategies for the more severely impaired patients.

During the second iteration feasibility study (chapter 7) we focused on including only moderately to mildly impaired patients, since they seemed the most suitable target group for the SCRIPT training system. These patients were able to complete the six weeks of training without severe difficulties, and six weeks of training resulted in additional improvements on activity level as well, compared to the first iteration (chapter 4). This suggests that the SCRIPT training system might indeed be suitable for moderately and mildly impaired stroke patients.

This patient-specific training approach actually covers a broader range of aspects, underlining the need for further examination into which factors are associated with better treatment outcomes. Within the SCRIPT study, we personalized the training to some extent by providing a custom-fit orthosis and selected games based on patient progress, but maybe we did not achieve its full potential with respect to the contents of the training and number of available games yet. The training could have been more personalized by taking into 
consideration other aspects, such as age, technical affinity, patients' preferences, coping style, or other personality characteristics, since individual stroke patients might differ in the way they think, feel and act.

In the current studies we included chronic stroke patients to diminish the influence of spontaneous recovery as much as possible, from a methodological point of view. Further, one of the main challenges of the SCRIPT training system was to explore if patients can and will use such a training system independently if they have the opportunity, even when they already finished their regular rehabilitation period, but still struggle with limitations in the performance of activities of daily living. Results of our studies show that the SCRIPT system is a feasible tool to enable self-administered practice at home in chronic stroke. In practice, such training should be provided at an earlier stage after stroke, for example in addition to inpatient rehabilitation. This will likely involve patients in the (sub)acute phase as well, where larger treatment effects would be expected considering optimal advantage of the time window for recovery. ${ }^{46,47}$

We included a wide range of patients' ages (34-80 years) into our studies, with diversity between people's technical affinity. In general, younger people have grown up with technologies such as internet and smartphones in a digital world, ${ }^{48}$ assuming higher technical affinity. Some of the older patients mentioned they were little scared to use the training system independently, were insecure if they used it correctly, and thought it was somewhat complicated donning and doffing the orthosis and starting a training session. People with less technical affinity might therefore show lower training duration than expected. Therefore, it is suggested to provide a user-friendly system and sufficient practice time for people with low technical affinity to get used to such a technology-supported system, to ensure confidence to use the system independently at home.

Some of the patients mentioned they had some concentration, motivation or planning problems due to their stroke. Their lack of discipline or perseverance could have resulted in a low training duration, since patients who are unmotivated will not exercise frequently or intensively enough. ${ }^{49,50}$ For future research, it is important to find methods to encourage patients with motivational problems to sustain training after stroke. Abovementioned examples show that many individual participant characteristics might play a role, indicating that a personalized training approach, taking into account characteristics and preferences of the individual patient, is highly recommended. These patient specific aspects could be considered for further development of such technology-supported training devices.

\section{OUTLOOK BEYOND SCRIPT AND FUTURE PERSPECTIVE}

The concept of SCRIPT, technology-supported self-administered training, could perfectly 
fit in innovative rehabilitation environments such as the Roessingh NovaLab, ${ }^{51}$ if it is usable, robust and practical in use. In such environments, newly developed devices can be rapidly brought to patients and healthcare professionals, and provide a rich environment of innovative rehabilitation possibilities with more variation and a targeted, more specific training. Such environments can serve as a starting place where people can learn to get acquainted with technology-supported systems. Perhaps this might result in becoming even more experienced in using such systems independently at home. In these environments, also other patient groups could potentially use such technology-supported devices for training. One can think of patients with multiple sclerosis, who also suffer from motor problems due to muscle weakness and coordination problems. ${ }^{52}$ These aspects can be considered for future applications of such technologies in practice.

Results of the chapters in this thesis show that technology-supported training at home have potential to comply with the increased demand of rehabilitation care. Several lessons have been learned during the development and evaluation of the SCRIPT system, which should be taken into account for future development and evaluation of such technology-supported devices.

- Technology-supported devices stressing active contribution of the patient seem most beneficial for improvements in arm function.

- The SCRIPT training system seems most suitable for moderately to mildly impaired stroke patients.

- $\quad$ The evaluation of the SCRIPT system was performed with chronic stroke patients. Technology-supported training is suggested for subacute patients as well, where even larger treatment effects would be expected.

- When developing technology-supported devices:

- Develop a robust, reliable and safe device, which patients can easily use independently. If need be, make use of a sufficient (supervised) orientation period, to let the patient get acquainted with the system for independent use.

- Take into account the weight of the device on the arm. Use an additional device supporting the arm against gravity when needed.

- Take into account specific patient characteristics for personalized training.

- It is important to keep patients motivated and challenged during training, because training duration is a major contributor to clinical changes. Examples are to provide exercises as games, offer a high variety of games, use competitive or cooperative games, and provide meaningful feedback.

- Independent training at home is feasible, but some kind of personal contact from a healthcare professional is highly appreciated and should not be replaced completely. 
- Provide challenging, functional movements as exercise training. Addressing both reaching and hand function in an integrated fashion are indicated as valuable targets for treatment applications to stimulate functional improvements after stroke.

As shown in this list, there are many possibilities to further explore and develop selfadministered technology-supported training systems for the arm and hand after stroke. However, we should not neglect personality aspects which could not be caught in technology development and depends on intrinsic patient motivation.

\section{"I believe I am actually using my hand more and better now in daily life. I noticed that my handwriting is getting better, especially the round letters. Yesterday, I even put on my shirt using both hands, which I never did after my stroke. Well, I used my right hand more than my left hand, but I still used it. But I have to say, it took me quite some time and effort to get there."}

This quote of a patient after the six weeks of training further emphasizes the importance of dedication and perseverance during training to obtain a high training duration and improve one's chance for clinical improvements. If we as researchers and developers could deliver usable technology to enhance functional training effectively in a highly motivational way, my advice for stroke patients starting a rehabilitation program would be: Roll up your sleeves, and go for it!

\section{REFERENCES}

1. Veerbeek JM, van Wegen $E$, van Peppen $R$, et al. What is the evidence for physical therapy poststroke? A systematic review and meta-analysis. PLoS One. 2014; 9: e87987.

2. Hatem SM, Saussez G, della Faille M, et al. Rehabilitation of Motor Function after Stroke: A Multiple Systematic Review Focused on Techniques to Stimulate Upper Extremity Recovery. Front Hum Neurosci. 2016; 10: 22.

3. Pollock A, Farmer SE, Brady MC, et al. Interventions for improving upper limb function after stroke. The Cochrane database of systematic reviews. 2014; 11: CD010820.

4. Mehrholz J, Pohl M, Platz T, Kugler J and Elsner B. Electromechanical and robot-assisted arm training for improving activities of daily living, arm function, and arm muscle strength after stroke. Cochrane Database Syst Rev. 2015; 11: CD006876.

5. Maciejasz P, Eschweiler J, Gerlach-Hahn K, Jansen-Troy A and Leonhardt S. A survey on robotic devices for upper limb rehabilitation. Journal of Neuroengineering and Rehabilitation. 2014; 11: 3.

6. Timmermans AA, Seelen HA, Willmann RD and Kingma H. Technology-assisted training of arm-hand skills in stroke: concepts on reacquisition of motor control and therapist guidelines for rehabilitation technology design. J Neuroeng Rehabil. 2009; 6: 1.

7. Jansen-Kosterink S, Vollenbroek-Hutten $\mathrm{M}$ and Hermens H. A Renewed Framework for the Evaluation of Telemedicine. eTELEMED 2016, The Eighth International Conference on eHealth, Telemedicine, and Social Medicine. Venice, Italy: IARIA, ThinkMind digital library, 2016, p. 57 to 62. 
8. Nasr N, Leon B, Mountain G, et al. The experience of living with stroke and using technology: opportunities to engage and co-design with end users. Disabil Rehabil Assist Technol. 2016; 11: 653-60.

9. Ates S, Lobo-Prat J, Lammertse P, van der Kooij H and Stienen AH. SCRIPT Passive Orthosis: Design and technical evaluation of the wrist and hand orthosis for rehabilitation training at home. IEEE International Conference on Rehabilitation Robotics. 2013; 2013: 1-6.

10. Ellis MD, Sukal T, DeMott T and Dewald JP. Augmenting clinical evaluation of hemiparetic arm movement with a laboratory-based quantitative measurement of kinematics as a function of limb loading. Neurorehabil Neural Repair. 2008; 22: 321-9.

11. Prange GB, Kottink Al, Buurke JH, et al. The effect of arm support combined with rehabilitation games on upper-extremity function in subacute stroke: a randomized controlled trial. Neurorehabil Neural Repair. 2015; 29: 174-82.

12. Dewald JP and Beer RF. Abnormal joint torque patterns in the paretic upper limb of subjects with hemiparesis. Muscle Nerve. 2001; 24: 273-83.

13. Ates S, Haarman CJW and Stienen AHA. SCRIPT passive orthosis: design of interactive hand and wrist exoskeleton for rehabilitation at home after stroke. Autonomous Robots. 2017; 41: 711-23.

14. Bangor A, Kortum PT and Miller JT. An Empirical Evaluation of the System Usability Scale. International Journal of Human-Computer Interaction. 2008; 24: 574-94.

15. Veerbeek JM, Langbroek-Amersfoort AC, van Wegen EE, Meskers CG and Kwakkel G. Effects of Robot-Assisted Therapy for the Upper Limb After Stroke. Neurorehabil Neural Repair. 2017; 31: 107-21.

16. Norouzi-Gheidari N, Archambault PS and Fung J. Effects of robot-assisted therapy on stroke rehabilitation in upper limbs: systematic review and meta-analysis of the literature. J Rehabil Res Dev. 2012; 49: 479-96.

17. Chen J, Nichols D, Brokaw EB and Lum PS. Home-based Therapy after Stroke Using the Hand Spring Operated Movement Enhancer (HandSOME). IEEE Trans Neural Syst Rehabil Eng. 2017.

18. Sivan M, Gallagher J, Makower S, et al. Home-based Computer Assisted Arm Rehabilitation (hCAAR) robotic device for upper limb exercise after stroke: results of a feasibility study in home setting. Journal of Neuroengineering and Rehabilitation. 2014; 11: 163.

19. Wittmann F, Held JP, Lambercy O, et al. Self-directed arm therapy at home after stroke with a sensor-based virtual reality training system. J Neuroeng Rehabil. 2016; 13: 75.

20. Wolf TJ, Chuh A, Floyd T, Mclnnis K and Williams E. Effectiveness of occupation-based interventions to improve areas of occupation and social participation after stroke: an evidence-based review. Am J Occup Ther. 2015; 69: 6901180060p1-11.

21. Kitago T and Krakauer JW. Chapter 8 - Motor learning principles for neurorehabilitation. In: Michael PB and David CG, (eds.). Handb Clin Neurol. Elsevier, 2013, p. 93-103.

22. Alt Murphy $M$, Willen $C$ and Sunnerhagen KS. Movement kinematics during a drinking task are associated with the activity capacity level after stroke. Neurorehabilitation and Neural Repair. 2012; 26: 1106-15.

23. Kwakkel G. Impact of intensity of practice after stroke: issues for consideration. Disabil Rehabil. 2006; 28: 823-30.

24. Kwakkel G. Intensity of practice after stroke: More is better. Schweizer Archiv für Neurologie und Psychiatrie. 2009; 7: 295-8.

25. Palmcrantz S, Borg J, Sommerfeld D, et al. An interactive distance solution for stroke rehabilitation in the home setting - A feasibility study. Inform Health Soc Care. 2016: 1-18.

26. Cunningham P, Turton AJ, Van Wijck F and Van Vliet P. Task-specific reach-to-grasp 
training after stroke: development and description of a home-based intervention. Clin Rehabil. 2016; 30: 731-40.

27. Donoso Brown EV, McCoy SW, Fechko AS, Price R, Gilbertson T and Moritz CT. Preliminary investigation of an electromyography-controlled video game as a home program for persons in the chronic phase of stroke recovery. Arch Phys Med Rehabil. 2014; 95: 14619.

28. Standen PJ, Threapleton K, Connell L, et al. Patients' Use of a Home-Based Virtual Reality System to Provide Rehabilitation of the Upper Limb Following Stroke. Phys Ther. 2014; 11: 11.

29. Maeder A, Poultney N, Morgan G and Lippiatt R. Patient Compliance in Home-Based Self-Care Telehealth Projects. J Telemed Telecare. 2015; 21: 439-42.

30. Putrino D. Telerehabilitation and emerging virtual reality approaches to stroke rehabilitation. Curr Opin Neurol. 2014; 27: 631-6.

31. Ronda G, Van Assema P and Brug J. Stages of change, psychological factors and awareness of physical activity levels in The Netherlands. Health Promot Int. 2001; 16: 305-14.

32. Donoso Brown EV and Fichter R. Home programs for upper extremity recovery poststroke: a survey of occupational therapy practitioners. Top Stroke Rehabil. 2017: 1-6.

33. Brewer BR, McDowell SK and Worthen-Chaudhari LC. Poststroke upper extremity rehabilitation: A review of robotic systems and clinical results. Topics in Stroke Rehabilitation. 2007; 14: 22-44.

34. Gorsic M, Cikajlo I and Novak D. Competitive and cooperative arm rehabilitation games played by a patient and unimpaired person: effects on motivation and exercise intensity. J Neuroeng Rehabil. 2017; 14: 23.

35. Vloothuis JD, Mulder M, Veerbeek JM, et al. Caregiver-mediated exercises for improving outcomes after stroke. Cochrane Database Syst Rev. 2016; 12: CD011058.

36. Wobma R, Nijland RH, Ket JC and Kwakkel G. Evidence for peer support in rehabilitation for individuals with acquired brain injury: A systematic review. J Rehabil Med. 2016; 48: 837-40.

37. Williams AB, Fennie KP, Bova CA, Burgess JD, Danvers KA and Dieckhaus KD. Home visits to improve adherence to highly active antiretroviral therapy: a randomized controlled trial. J Acquir Immune Defic Syndr. 2006; 42: 314-21.

38. Currie M, Philip LJ and Roberts A. Attitudes towards the use and acceptance of eHealth technologies: a case study of older adults living with chronic pain and implications for rural healthcare. BMC Health Serv Res. 2015; 15: 162.

39. Reinkensmeyer DJ and Boninger ML. Technologies and combination therapies for enhancing movement training for people with a disability. J Neuroeng Rehabil. 2012; 9: 17.

40. Subramanian SK, Massie CL, Malcolm MP and Levin MF. Does provision of extrinsic feedback result in improved motor learning in the upper limb poststroke? A systematic review of the evidence. Neurorehabil Neural Repair. 2010; 24: 113-24.

41. Wolf SL, Kwakkel G, Bayley M, McDonnell MN and Upper Extremity Stroke Algorithm Working G. Best practice for arm recovery post stroke: an international application. Physiotherapy. 2016; 102: 1-4.

42. Hurdowar A, Graham ID, Bayley M, Harrison M, Wood-Dauphinee S and Bhogal S. Quality of stroke rehabilitation clinical practice guidelines. J Eval Clin Pract. 2007; 13: 657-64.

43. Amirabdollahian F, Ates S, Basteris A, et al. Design, development and deployment of a hand/wrist exoskeleton for home-based rehabilitation after stroke - SCRIPT project. Robotica. 2014; 32: 1331-46. 
44. Basteris A, Nijenhuis SM, Buurke JH, Prange GB and Amirabdollahian F. Lag-lead based assessment and adaptation of exercise speed for stroke survivors. Robotics and Autonomous Systems. 2015; 73: 144-54.

45. Stein J, Krebs HI, Frontera WR, Fasoli SE, Hughes R and Hogan N. Comparison of two techniques of robot-aided upper limb exercise training after stroke. Am J Phys Med Rehabil. 2004; 83: 720-8.

46. Kwakkel G, Kollen BJ and Wagenaar RC. Long term effects of intensity of upper and lower limb training after stroke: a randomised trial. J Neurol Neurosurg Psychiatry. 2002; 72: 473-9.

47. Langhorne P, Bernhardt J and Kwakkel G. Stroke rehabilitation. Lancet. 2011; 377: 1693702.

48. Prensky M. Digital natives, digital immigrants part 1. On the horizon. 2001; 9: 1-6.

49. Maclean N, Pound P, Wolfe C and Rudd A. The concept of patient motivation: a qualitative analysis of stroke professionals' attitudes. Stroke; a journal of cerebral circulation. 2002; 33: 444-8.

50. Maclean $\mathrm{N}$ and Pound P. A critical review of the concept of patient motivation in the literature on physical rehabilitation. Soc Sci Med. 2000; 50: 495-506.

51. Krabbe D and Nijland M. Novalab: de brug tussen wetenschap en praktijk. CVA kennisnetwerk Nederland - Mee(r) doen na een CVA. Zeist, the Netherlands2015.

52. Compston A and Coles A. Multiple sclerosis. Lancet. 2008; 372: 1502-17. 
General discussion 



\title{
\&
}

\author{
Summary \\ Samenvatting \\ Dankwoord \\ About the author \\ Progress range
}




\section{SUMMARY}

Chapter 1 provided a short overview of the background of stroke and rehabilitation after stroke. Many stroke patients have an impaired arm and hand function, which limits them in the performance of activities of daily living independently. In the long run, this may result in learned non-use of the affected arm and hand in daily life. To overcome these impairments, it is important to maintain the use of this arm and hand. There are several aspects which are important to stimulate restoration of arm and hand function. Exercises should be provided in a high intensity with many repetitions, with active involvement of the patient, preferably in a motivating environment. Technologies, such as rehabilitation robotics, have the opportunity to involve many of these aspects, and they can assist the therapist in such an intensive therapy. The use of rehabilitation robotics has been shown to be effective for the hemiparetic arm. However, evidence of the transfer of robotic training effects to activities in daily life remains limited. More insight is needed into the best therapy strategy and adequate design of robotic upper extremity devices. A next step would be to provide such technologies at home, to support self-administered training, without requiring continuous therapist involvement. In the Supervised Care and Rehabilitation Involving Personal Telerobotics project (SCRIPT) we combined both the technology and telemedicine aspects. This thesis aims to evaluate the global impact (in terms of feasibility and potential clinical effects) of self-administered technology-supported, functional training of the arm and hand at home in chronic stroke patients, to enhance independent, motivational and active exercise.

We started with a literature review in chapter $\mathbf{2}$, to provide an overview of existing and most effective training modalities in robot-mediated upper extremity training after stroke. The review shows that most of the studies so far focus on training of the proximal arm in chronic stroke patients, although integration of distal with proximal arm training might be most effective to enhance functional gains. Studies often refer to active, active-assisted and passive interaction training modalities, without providing information regarding the mechanism about the physical human-robot interaction. Features stressing active contribution by the patient, such as EMG-modulated forces or a pushing force in combination with springdamper guidance, may be beneficial for improvements in arm function. Therefore, active contribution of the patient during training, involving both proximal with distal arm movements, was incorporated within the SCRIPT training study.

One of the components of the SCRIPT training system is a passive actuated dynamic wrist and hand orthosis, which physically interacted with the arm and hand. In chapter $\mathbf{3}$ we assessed the direct effects of this orthosis on hand and arm movements during the performance of a reach and grasp task in ten mildly to moderately impaired chronic stroke patients. The use of the orthosis had a positive direct influence on maximum hand opening, but negative 
direct influence on the trunk displacement and elbow range of motion, probably due to the weight of the orthosis on the arm. The use of an additional device supporting the arm against gravity was added when using such an orthosis for training purposes, to counteract potential compensatory trunk movements. The complete training system was evaluated at stroke patients' homes in chapter 4. This training system involved the passive actuated wrist and hand orthosis, the Saebo Mobile Arm Support, and a computer containing a motivational user interface within a gaming environment for training of arm, wrist and hand movements. This training system showed to be a feasible tool to enable self-administered practice: chronic stroke patients were able to use the training system independently at home. They were motivated to train, although some usability issues were identified that needed further attention. Upper extremity function and quality of life improved after six weeks of training, although this was not transferred to improvements on activity level.

The SCRIPT training system was compared to a control group performing conventional exercises from an exercise book, in chapter $\mathbf{5}$. The control group reported a higher training duration, but perceived motivation was equal for both groups. The effects of technologysupported arm and hand training seemed comparable to conventional arm and hand exercises at home, since no differences in clinical outcomes over training between groups were found. A positive correlation with training duration and training-induced changes in clinical outcome measures was found, indicating that training duration in itself seems a major contributor to arm and hand function improvements. It is assumed that self-administered training at home is a good solution to provoke a high training dose, because this will remove training constraints since patients are not dependent of therapist availability.

We aimed to gain more insight into underlying mechanisms of recovery and best treatment approach by investigating relationships in chapter 6 . We investigated relationships between an extensive set of objective movement execution kinematics of the upper extremity and grip strength with clinical outcome measures in chronic stroke patients. Moderate to strong relationships for most movement execution parameters with post-stroke arm function (FuglMeyer assessment) and activities (Action Research Arm Test) were found, with strongest contributions for grip strength and elbow excursion. Following these relations, addressing both reaching and hand function are indicated as valuable targets for (technologysupported) treatment applications to stimulate functional improvements after stroke. This was targeted within chapter 7 , in which the usability issues found during the first iteration of the system were advanced in the second iteration training system. This second iteration involved an updated wrist and hand supporting orthosis with improved user interface and larger variety of more functional games incorporating also versatile grasping exercises. This system was evaluated on feasibility in a new group of chronic stroke patients. Also here, the training system was motivational and usable. Moreover, training for six weeks resulted in 
improvements on both arm and hand function and dexterity.

Based on the findings of the previous chapters, chapter 8 discussed main findings and recommendations for future research. Results of the chapters in this thesis show that self-administered technology-supported arm and hand training at home have potential to comply with the increased demand of rehabilitation care. To further enhance this potential of technology-supported devices in future, it is important to develop a robust, reliable and safe device, which patients can easily use independently. Besides, patients should be kept motivated and challenged to training, because training duration is a major contributor to clinical changes. And finally, the contents of the training should address both reaching and hand function, since they are indicated as valuable targets for treatment applications to stimulate functional improvements after stroke. 


\section{SAMENVATTING}

In hoofdstuk 1 wordt een overzicht gegeven van de achtergrond van een cerebrovasculair accident (CVA), ook wel beroerte, en de revalidatie na een beroerte. Na een beroerte hebben veel patiënten een enkelzijdige verlamming van de arm en hand. Dit kan problemen opleveren in het zelfstandig uitvoeren van activiteiten in het dagelijks leven. Op de lange termijn kan dit leiden tot het zogenaamde 'learned non-use', dat wil zeggen dat de patiënt als het ware 'leert' de aangedane arm en hand niet te betrekken in het uitvoeren van functionele activiteiten. Om deze beperkingen te verminderen is het belangrijk om de aangedane arm en hand te blijven gebruiken. Er zijn een aantal aspecten binnen therapeutische interventies die het mogelijk maken om herstel van arm- en handfunctie na een beroerte te stimuleren. Oefeningen zullen moeten worden uitgevoerd in een hoge trainingsintensiteit, met veel herhalingen, met actieve initiatie en uitvoering van bewegingen door de patiënt zelf, bij voorkeur in een motiverende trainingsomgeving. Met behulp van technologische innovaties, zoals revalidatierobots, kunnen veel van deze aspecten worden toegepast om intensieve revalidatie van de arm en hand mogelijk te maken. Daarnaast kunnen revalidatierobots therapeuten ondersteunen bij een dergelijke intensieve therapie. Het gebruik van robotica in de revalidatie is veelbelovend voor het verbeteren van de armfunctie na een beroerte. Er is echter nog beperkt bewijs dat het gebruik van revalidatierobots resulteert in verbeteringen op activiteitenniveau en voor daadwerkelijk beter gebruik van de arm en hand in het dagelijks leven. Meer inzicht is nodig in de meest effectieve trainingstoepassingen en een adequaat ontwerp van revalidatietechnologie voor de arm en hand. Een volgende stap is om een dergelijke technologie in de thuissituatie aan te bieden waarbij patiënten zelfstandig kunnen oefenen, zonder directe aanwezigheid van een therapeut. Binnen het Europese Supervised Care \& Rehabilitation Involving Personal Telerobotics (SCRIPT) project hebben we technologie met zorg op afstand kunnen combineren. Het doel van het onderzoek beschreven in dit proefschrift is een beter inzicht te verkrijgen (in termen van haalbaarheid en potentiele klinische effecten) van zelfstandig uitgevoerde functionele training van de arm en hand in de thuisomgeving dat wordt ondersteund door technologie, in de chronische fase na een beroerte.

Als eerste is in hoofdstuk 2 een literatuuronderzoek (review) verricht om een overzicht te verkrijgen van de bestaande en meest effectieve trainingsmodaliteiten binnen robot ondersteunende training van de arm en hand na een beroerte. Uit de review blijkt dat de meeste onderzoeken tot dusver gericht waren op het trainen van de proximale arm in de chronische fase na een beroerte, hoewel de combinatie van zowel proximale met distale arm en hand training het meest effectief lijkt voor verbeteringen op armfunctie en activiteitenniveau. Studies beschrijven vaak actieve, actief-ondersteunend, en passieve modus als trainingsmodaliteiten, maar geven geen nadere informatie betreffende welke mechanismen met betrekking tot mens-robot interactie een rol spelen. Mechanismen die 
een actieve bijdrage van de patiënt benadrukken in de training, zoals elektromyografisch gemoduleerde krachten, of duwkracht in combinatie met veerdempergeleiding, leken het meest voordelig in het verbeteren van armfunctie. Daarom werd de actieve bijdrage van de patiënt tijdens training, en het trainen van zowel de proximale als distale arm en hand meegenomen in het ontwikkelen van de SCRIPT trainingsstudie.

Een van de onderdelen van het SCRIPT trainingssysteem is een passief-geactueerde dynamische pols en hand orthese, welke gedragen werd om de onderarm en hand. In hoofdstuk 3 hebben we het directe effect van deze orthese op hand en arm bewegingen onderzocht tijdens het uitvoeren van een reik en grijptaak in tien licht tot matig aangedane chronische CVA patiënten. Het gebruik van de orthese had een positieve directe invloed op maximale hand opening, maar negatieve directe invloed op romp verplaatsing en de bewegingsuitslag van de elleboog. Dit kwam mogelijk door het gewicht van de orthese aan de arm. Het gebruik van een aanvullend apparaat dat de arm ondersteunt tegen de zwaartekracht werd toegevoegd voor trainingsdoeleinden, om potentiele compensatoire bewegingen van de romp tegen te gaan. Het complete SCRIPT trainingssysteem is daarna geëvalueerd bij CVA patiënten thuis (hoofdstuk 4). Dit trainingssysteem bestaat uit de passief-geactueerde pols en hand orthese, de Saebo mobiele arm ondersteuner, een computer met motiverende gebruiksomgeving inclusief spellen om arm, pols, en hand bewegingen te oefenen. Dit trainingssysteem bleek een haalbaar middel om zelfstandig te oefenen: chronische CVA patiënten waren in staat het trainingssysteem zelfstandig thuis te gebruiken. Ze waren gemotiveerd om te trainen, hoewel er nog wel enige verbeterpunten in de bruikbaarheid van het systeem naar voren kwamen. Armfunctie en kwaliteit van leven verbeterden na zes weken training, maar er waren geen verbeteringen te zien op activiteitenniveau.

In hoofdstuk $\mathbf{5}$ is het gebruik van het SCRIPT trainingssysteem bij patiënten na een beroerte vergeleken met een controlegroep waarbij patiënten hebben getraind met conventionele oefeningen uit een oefengids. De controlegroep rapporteerde een hogere trainingsduur, maar de waargenomen motivatie was gelijk in beide groepen. Het effect van arm- en handtraining thuis dat wordt ondersteund door technologie bleek vergelijkbaar met conventionele arm en hand oefeningen, aangezien er geen verschillen in klinische uitkomstmaten tussen beide groepen zijn gevonden. Er werd een positieve correlatie van trainingsduur met traininggerelateerde veranderingen in de klinische uitkomstmaten gevonden. Op dit moment lijkt trainingsduur op zich een belangrijke factor te zijn voor klinische vooruitgang in arm- en handfunctie. Er wordt verondersteld dat zelfstandige training thuis een geschikte oplossing is om een hoge trainingsduur te behalen, omdat patiënten niet afhankelijk zijn van de beschikbaarheid van een therapeut. 
Het doel van hoofdstuk 6 was om meer inzicht te verkrijgen in onderliggende mechanismen van herstel en beste trainingsaanpak. We onderzochten relaties tussen een uitgebreide set van objectieve kinematische bewegingsparameters van de bovenste extremiteit en knijpkracht met klinische uitkomstmaten, bij chronische CVA patiënten. Er werden matige tot sterke relaties voor de meeste bewegingsparameters met arm functie (Fugl-Meyer assessment) en activiteitenniveau (Action Research Arm Test) gevonden, waarbij knijpkracht en de bewegingsuitslag van de elleboog de grootste bijdragen leverden. Het toepassen van zowel reikbewegingen als hand functie binnen een (technologie ondersteunende) trainingssessie wordt daarom aanbevolen om functioneel herstel na een beroerte te bevorderen. Dit was een van de doelstellingen om mee te nemen in hoofdstuk 7, waarin ook andere verbeterpunten in de bruikbaarheid van het eerste trainingssysteem werden doorgevoerd in een geavanceerdere tweede versie van het trainingssysteem. Deze versie bestond uit een verbeterde pols en hand orthese met bijgewerkte gebruikersomgeving en groter aanbod aan functionele spellen, waaronder ook veelzijdige grijpoefeningen. Dit vernieuwde systeem werd opnieuw geëvalueerd op haalbaarheid in een nieuwe groep patiënten in de chronische fase na een beroerte. Wederom werd de training als motiverend en bruikbaar beoordeeld. Daarnaast werden na zes weken training zowel verbeteringen op armfunctie als handvaardigheid gevonden.

Gebaseerd op de bevindingen in voorgaande hoofdstukken, werden in hoofdstuk 8 de belangrijkste bevindingen en aanbevelingen voor toekomstig onderzoek besproken. De resultaten uit de hoofdstukken in dit proefschrift laten zien dat het zelfstandig uitvoeren van functionele training van de arm en hand thuis dat wordt ondersteund door technologie potentie heeft om te voldoen aan de groeiende vraag naar revalidatiezorg. Om een dergelijk trainingsapparaat en training nog verder te verbeteren in de toekomst, is het belangrijk om robuuste, betrouwbare en veilige technologieën te blijven ontwikkelen, die patiënten eenvoudig zelfstandig kunnen gebruiken. Daarnaast is het belangrijk dat patiënten gemotiveerd en uitgedaagd blijven om te trainen, aangezien trainingsduur een belangrijke factor lijkt te zijn voor klinische vooruitgang in arm- en handfunctie. Tot slot, de inhoud van de training moet zich richten op zowel het oefenen van reikbewegingen als hand functie, omdat optimale integratie hiervan in de training functioneel herstel na een beroerte lijkt te bevorderen. 


\section{DANKWOORD}

Hier is het dan, mijn proefschrift. Het is eindelijk af! Het kostte wat tijd en moeite om dit naast een nieuwe baan te voltooien, maar ik ben blij dat ik het af heb kunnen ronden. Dit was niet gelukt zonder de hulp en steun van vele mensen. Ik wil iedereen hierbij dan ook heel erg bedanken.

Allereerst mijn (co)-promotoren. Ik ben erg dankbaar dat ik deel mocht uitmaken van het internationale SCRIPT project. Hierdoor heb ik mezelf erg goed kunnen ontwikkelen, en mijn Engels eens goed bij kunnen spijkeren. Ook heb ik geleerd wat het is om samen te werken met een diversiteit aan mensen met een verschillende achtergrond, en de technische ontwikkeling van een trainingssysteem; wat niet altijd zonder slag of stoot gaat. Ik ben dankbaar dat ik deze mogelijkheid heb gekregen, met als resultaat het proefschrift dat nu voor me ligt.

Hans, ik heb veel van je geleerd. Je kritische blik op het SCRIPT systeem heeft me goed aan het denken gezet en mij ook kritisch leren kijken. Hoewel het me soms wat tijd heeft gekost om je commentaar op artikelen te ontcijferen, was dit erg nuttige feedback en heb ik nu een mooi aantal publicaties liggen. Een van de hoogtepunten (zowel letterlijk als figuurlijk) was misschien wel het tripje naar Singapore, waar we onder het genot van een lekkere biercocktail met een mooi uitzicht over de baai nog eens konden napraten over onze ervaringen van de stad en het congres.

Jaap, bedankt voor je begeleiding en wetenschappelijke input. Ik heb het erg gewaardeerd dat je altijd betrokken bent, zowel binnen als buiten het werk om. De werkoverleggen heb ik als heel prettig ervaren, waarin je een verhelderende blik gaf en waarin de kern van het verhaal mooi samengevat werd in een paar zinnen, daar waar ik dan al een tijdje mee aan het stoeien was. Je hebt me laten zien hoe ik me de afgelopen jaren heb ontwikkeld. Heel erg bedankt voor al je steun, en wat leuk dat ik je op de valreep ook promotor mag noemen!

Gerdienke, heel erg bedankt voor al je hulp en de dagelijkse begeleiding. Ik heb erg veel gehad aan je kritische blik op mijn artikelen, iets wat ik misschien zelf wel een beetje van je overgenomen heb. Het was prettig dat ik altijd bij je binnen kon lopen voor tips, een korte vraag of gewoon om frustraties te uitten. Er ging soms heel wat overlegtijd op aan gesprekken over zaken buiten het werk, maar daar heb ik erg van genoten. Ook hebben we heel wat tripjes gemaakt naar Europese steden, voor congresbezoek of SCRIPT meetings. Leuk om rondom de werkzaamheden samen de stad te verkennen!

I would like to thank the members of my graduation committee for the willingness to read and evaluate my thesis and be part of my PhD defence. 
SCRIPT project members from the University of Hertfordshire, Roessingh Research and Development, University of Twente, R.U.Robots Limited, University of Sheffield, MOOG BV, San Raffaele S.p.A and User Interface Design $\mathrm{GmbH}$ : Thank you very much for the nice cooperation during the SCRIPT project, which resulted in this thesis. I really enjoyed the SCRIPT meetings which gave me the opportunity to travel a lot around Europe. A special thanks to the UH-team for their heartwarming hospitality at Hatfield and tour through London during the Short Term Scientific Mission. I felt extremely welcome!

Ik heb een bijdrage mogen leveren aan verschillende projecten, waarbij ik samengewerkt heb met vele onderzoekers, revalidatieartsen en therapeuten. Allemaal bedankt voor jullie inzet en hulp, o.a. bij de inclusie van proefpersonen. Ook een grote dank gaat uit naar deze proefpersonen, zonder jullie had ik de metingen niet kunnen uitvoeren en de artikelen niet kunnen schrijven. Bedankt voor jullie inzet, jullie hebben heel wat inspanning vertoont tijdens de trainingen en geduld moeten hebben tijdens de metingen.

Ook dank aan mijn paranimfen, Florentine en Anne. Leuk dat jullie tijdens de verdediging aan mijn zijde willen staan! Anne, ik vond het fijn om met je te sparren over ons onderzoek, en gezellig bij te kletsen over zaken naast het werk. Ook al zie ik je niet meer zo vaak, het blijft leuk om tijdens mijn meetings bij RRD met je bij te kletsen! Leendert, bedankt voor je technische ondersteuning en hulp met mijn Matlab script. Erg fijn dat je me hierbij hebt willen helpen! Stephanie, leuk dat ik al die jaren een kamer met je heb mogen delen. Inger en Gerda, bedankt voor jullie hulp als er weer eens een afspraak moest worden gepland of iets geregeld moest worden.

En natuurlijk ook alle andere oud-collega's van RRD: Ik heb erg genoten van de gezelligheid en spontaniteit, waardoor ik het dan ook jammer vond om weg te gaan. Ik denk met veel plezier terug aan de (cluster)uitjes, de Laatste-Donderdag-van-de-Maand-Borrels, squashtoernooien, lunchwandelingen en praatjes in de koffiehoek. Bedankt allemaal dat jullie mijn verblijf zo prettig hebben gemaakt. Ook wil ik graag de studenten bedanken die ik de jaren bij RRD heb mogen begeleiden en daardoor hebben mogen bijdragen aan mijn promotieonderzoek.

Ook aan mijn oud-collega-onderzoekers van het Radboudumc en leden van het Room with a View team. Fijn dat jullie me ook in Nijmegen thuis hebben laten voelen. Ook al deed ik geen promotie-onderzoek bij jullie, het is fijn om bij mensen te zitten die in dezelfde situatie zitten en ervaringen met elkaar te delen. Jullie gezelligheid tijdens de lunch, het taart eten, de afdelingsuitjes, de gesprekken in de kantoortuin en de vele borrels (ja die ga ik inderdaad missen...), maakten de reis naar Nijmegen meer dan waard! 
Huidige collega's van het SKB, fijn dat jullie mij ook hier al snel thuis laten voelen. Ik waardeer het dat ik de kans krijg om mijn proefschrift af te ronden en weer te kunnen starten met nieuwe uitdagingen!

Familie en vrienden, bedankt voor jullie belangstelling en steun, en natuurlijk de gezellige afleiding op zijn tijd. Door op de juiste momenten tijd vrij te maken voor jullie ging het afronden van het proefschrift er misschien niet sneller om, maar dat maakte het wel een stuk aangenamer!

En natuurlijk Wouter. Het afronden van dit proefschrift heeft de nodige stress gegeven. Het is dan ook erg prettig om na een dag werken thuis te komen en zo aan tafel te kunnen schuiven, en mijn ervaringen van de dag met je te delen. Je bent altijd een grote steun voor mij en ik geniet van de leuke dingen die we samen doen. Fijn dat je in mijn leven bent! 


\section{ABOUt THE AUTHOR}

Sharon Nijenhuis was born in Lichtenvoorde, the Netherlands, on October 25th 1986. In 2005 she received her high school diploma from the R.K.S.G. Marianum in Groenlo, and moved to Nijmegen to start a study in Biomedical Sciences at the Radboud University Nijmegen. After receiving her Bachelors degree, she focused on the specialization Human Movement Sciences and followed an internship at Roessingh Research and Development (RRD) in Enschede. After her graduation in 2011, she continued to work for RRD as a PhD student (2011-2016) at the European Supervised Care \& Rehabiliation Involving Personal Telerobotics (SCRIPT) project. The results of this research are presented in this thesis.

After her work at RRD, Sharon was employed as a research coordinator (2016-2017) at the innovation project 'Room with a view' at the Radboudumc, department of surgery, Nijmegen. This project focused on the development of a personalized patient environment using smart and simple technologies, contributing to recovery and optimal wellbeing, with a focus on minimal stress, optimal pain reduction, good sleep and improved mobility.

Currently, Sharon is employed as a biomedical technologist at Streekziekenhuis Koningin Beatrix in Winterswijk. There she has an important role in innovation, quality assurance and risk management of medical devices. She contributes to optimal and safe deployment of medical technology within the hospital with focus areas: sterile instrumentation, endoscopy, implants and medical disposables.

\section{Journal publications}

- Nijenhuis SM, Prange-Lasonder GB, Stienen AH, Rietman JS and Buurke JH. Effects of training with a passive hand orthosis and games at home in chronic stroke: a pilot randomised controlled trial. Clinical Rehabilitation. 2017; 31: 207-16.

- Nijenhuis SM, Prange GB, Stienen A, Rietman JS and Buurke J. Effect van arm- en handtraining thuis met behulp van technologie in de chronische fase na een beroerte. Nederlands tijdschrift voor revalidatiegeneeskunde (NTR). 2016; 38: 195 - 200.

- Nijenhuis SM, Prange GB, Amirabdollahian F, et al. Feasibility study into self-administered training at home using an arm and hand device with motivational gaming environment in chronic stroke. Journal of Neuroengineering and Rehabilitation. 2015; 12: 89.

- Nasr N, Leon B, Mountain G, Nijenhuis SM, Prange G, Sale P and Amirabdollahian F. The experience of living with stroke and using technology: opportunities to engage and codesign with end users. Disabil Rehabil Assist Technol. 2016; 11: 653-60.

- Basteris A, Nijenhuis SM, Stienen AH, Buurke JH, Prange GB and Amirabdollahian F. Training modalities in robot-mediated upper limb rehabilitation in stroke: a framework for classification based on a systematic review. Journal of Neuroengineering and Rehabilitation. 2014; 11: 111.

- Basteris A, Nijenhuis SM, Buurke JH, Prange GB and Amirabdollahian F. Lag-lead based assessment and adaptation of exercise speed for stroke survivors. Robotics and Autonomous Systems. 2015; 73: 144-54. 
- Amirabdollahian F, Ates S, Basteris A, Cesario A, Buurke J, Hermens H, Hofs D, Johansson E, Mountain G, Nasr N, Nijenhuis S, Prange G, Rahman N, Sale P, Schätzlein F, van Schooten B and Stienen A. Design, development and deployment of a hand/wrist exoskeleton for home-based rehabilitation after stroke - SCRIPT project. Robotica. 2014; 32: 1331-46.

- Leon B, Basteris A, Infarinato F, Sale P, Nijenhuis S, Prange G and Amirabdollahian F. Grasps recognition and evaluation of stroke patients for supporting rehabilitation therapy. Biomed Res Int. 2014; 2014: 318016.

\section{Submitted for publication}

- $\quad$ Nijenhuis SM, Prange-Lasonder GB, Fleuren JFM, Wagenaar J, Buurke JH, Rietman JS. Strong relations of elbow excursion and grip strength with post stroke arm function and activities: should we aim for this in technology-supported training? Submitted to: Journal of Rehabilitation and Assistive Technologies Engineering

\section{Book chapters}

- Ortiz-Catalan M, Nijenhuis S, Ambrosch K, Bovend'Eerdt T, Koenig S and Lange B. Virtual Reality. In: Pons JL and Torricelli D, (eds.). Emerging Therapies in Neurorehabilitation. Springer Berlin Heidelberg, 2014, p. 249-65.

\section{Conference contributions}

- Nijenhuis SM. Room with a view. Verpleegkundig symposium, lustrum congres heelkunde 60 jaar verpleegkundige zorg in het Radboudumc: 'verleden-heden-toekomst'. 12 October 2016, Nijmegen, the Netherlands.

- Nijenhuis SM. Upper extremity training at home after stroke. Symposium 25 years Roessingh Research and Development: Taking care of the Future 'Enhanced Technology for Rehabilitation'. 22 September 2016, Hengelo, the Netherlands.

- Nijenhuis SM, Prange-Lasonder GB, Amirabdollahian F, Infarinato F, Buurke JH and Rietman JS. Feasibility of a Second Iteration Wrist and Hand Supported Training System for Self-administered Training at Home in Chronic Stroke. The Eighth International Conference on eHealth, Telemedicine, and Social Medicine (eTELEMED). 24-28 April 2016, Venice, Italy. In: IARIA, ThinkMind digital library, p. 51-56. ISBN: 978-1-61208470-1

- Nijenhuis SM, Prange GB, Sale P, Nasr N, Buurke JH and Rietman JS. Home-based technology-supported training compared to conventional arm and hand exercises after stroke. Dutch Congress of Rehabilitation Medicine (DCRM). 5-7 November 2015, Rotterdam, the Netherlands. (Winner best abstract presentation). In: Abstracts of meeting DCRM 5-7 November 2015. Clinical Rehabilitation. 2016; 30: 719-25.

- Nijenhuis SM, Prange GB, Rietman JS, Buurke JH. Feasibility of home-based technologysupported arm and hand training after stroke. Jubileumcongres Nederlandse Vereniging voor Fysiotherapie in de Geriatrie (NVFG) 'Technologie: Nieuw voor Oud'. 25 September 2015, Utrecht, the Netherlands.

- Nijenhuis SM. Technology-supported versus usual arm and hand training at home in chronic stroke. VvBN PhD-day 2015 'Enabling technology for human functioning'. 24 September 2015, Enschede, the Netherlands.

- Nijenhuis SM, Prange GB, Stienen AHA, Buurke JH and Rietman JS. Direct effect of a dynamic wrist and hand orthosis on reach and grasp kinematics in chronic stroke. IEEE International Conference on Rehabilitation Robotics (ICORR). 11-14 August 2015, Singapore. In: IEEE Xplore Digital Library, p. 404-9. DOI:10.1109/ICORR.2015.7281233. 
- $\quad$ Radder B, Kottink AIR, van der Vaart N, Oosting D, Buurke JH, Nijenhuis SM, Prange GB and Rietman JS. User-centred Input for a Wearable Soft-Robotic Glove Supporting Hand Function in Daily Life. IEEE International Conference on Rehabilitation Robotics (ICORR). 11-14 August 2015, Singapore. In: IEEE Xplore Digital Library, p. 502-7. DOI:10.1109/ ICORR.2015.7281249

- Prange GB, Smulders LC, van Wijngaarden J, Lijbers GJ, Nijenhuis SM, Veltink PH, Buurke JH and Stienen AHA. User Requirements for Assistance of the Supporting Hand in Bimanual Daily Activities via a Robotic Glove for Severely Affected Stroke Patients. IEEE International Conference on Rehabilitation Robotics (ICORR). 11-14 August 2015, Singapore. In: IEEE Xplore Digital Library, p. 357-61. DOI:10.1109/ICORR.2015.7281225

- Nijenhuis SM, Prange GB, Wagenaar J, Buurke JH, Rietman JS. Feasibility of technology supported arm and hand training compared with usual arm and hand training at home in chronic stroke. International Congress on NeuroRehabilitation and Neural Repair. 2122 May 2015, Maastricht, the Netherlands.

- Nijenhuis SM, Prange GB, Sale P, Nasr N, Buurke JH, Rietman JS. Evaluation of a homebased technology-supported arm/hand training system after stroke. Dutch Congress of Rehabilitation Medicine (DCRM). 6-7 November 2014, Rotterdam, the Netherlands. In: Abstracts of the Dutch Congress of Rehabilitation Medicine (DCRM) meeting. Clinical Rehabilitation. 2015; 29: 306-11.

- Prange GB, Nijenhuis SM, Sale P, Cesario A, Nasr N, Mountain G, Stienen, AH, Amirabdollahian $\mathrm{F}$, Buurke $\mathrm{JH}$. Adherence and arm function improvements with homebased distal arm training using robotics and gaming after stroke. Design of Medical Devices Conference (DMD). 22-24 October 2014, Delft, the Netherlands.

- Prange GB, Nijenhuis SM, Sale P, Cesario A, Nasr N, Mountain G, Amirabdollahian F, Buurke JH. Preliminary findings of feasibility and compliance of technology-supported distal arm training at home after stroke. International Conference on NeuroRehabilitation (ICNR). 24-26 June 2014, Aalborg, Denmark. In: Jensen W, Andersen OK and Akay M, (eds.). Replace, Repair, Restore, Relieve - Bridging Clinical and Engineering Solutions in Neurorehabilitation. Springer International Publishing, 2014, p. 665-73. DOI:10.1007/978-3-319-08072-7_94

- Prange GB, Nijenhuis SM, Kersten SM, Stienen AHA and Rietman JS. Exploring Relations between Functional Task Kinematics and Clinical Assessment of Arm Function and Dexterity Post-stroke. International Conference on NeuroRehabilitation (ICNR). 24-26 June 2014, Aalborg, Denmark. In: Jensen W, Andersen OK and Akay M, (eds.). Replace, Repair, Restore, Relieve - Bridging Clinical and Engineering Solutions in Neurorehabilitation. Springer International Publishing, 2014, p. 675-84. DOI:10.1007/978-3-319-08072-7_95

- $\quad$ Ates S, Leon B, Basteris A, Nijenhuis S, Nasr N, Sale P, Cesario A, Amirabdollahian F and Stienen AHA. Technical evaluation of and clinical experiences with the SCRIPT passive wrist and hand orthosis. 7th International Conference on Human System Interactions (HSI). 7th ed, 16-18 June 2014, Lisbon, Portugal. In: IEEE Xplore Digital Library, p. 18893. DOI:10.1109/hsi.2014.6860472

- Nijenhuis SM, Prange GB, Sale P, Cesario A, Amirabdollahian F, Rietman JS, Buurke $\mathrm{JH}$. Feasibility of a robotic arm/hand training system at home after stroke. 8th World Congress for NeuroRehabilitation (WCNR); 8-12 April 2014, Istanbul, Turkey.

- Nijenhuis SM, Prange GB, Schäfer J, Rietman JS, Buurke JH. Feasibility of a personalized arm/hand training system for use at home after stroke: results so far. International NeuroRehabilitation Symposium (INRS). 11-13 September 2013, Zürich, Switzerland.

- Nijenhuis SM, Prange GB, Rietman JS, Buurke JH. Design of the SCRIPT robotic device for arm and hand training to improve activities during home-based robotic training in chronic stroke patient. COST symposium Rehabilitation Robotics. 8-9 April 2013, University of Twente, Enschede, the Netherlands. 
- Nijenhuis SM, Prange GB, Rietman JS, Buurke JH. Design of the SCRIPT robotic device for arm and hand training to improve activities during home-based robotic training in chronic stroke patient. Summer school on Neurorehabilitation "Emerging therapies". 16-21 September 2012, Monasterio de Piedra, Zaragoza, Spain. 


\section{Progress RANGe}

The following publications have been published in the 'Progress in rehabilitation science' range by Roessingh Research and Development, Enschede, the Netherlands. Copies can be ordered, when available, via info@rrd.nl.

Progress 1: Pot JWGA, Boer H, van Harten WH, Hermens HJ, Seydel ER. Comprehensive Need-Assessment. Ontwikkeling van een meetinstrument voor zorgbehoeften en kwaliteitsbeoordeling door patiënten. Roessingh Research and Development, The Netherlands, September 1994, ISBN 90-25452-01-2

Progress 2: van Leerdam NGA, Hermens HJ. Revalidatietechnologie in Euregio. Roessingh Research and Development, The Netherlands, July 1995, ISBN 90-75452-02-0

Progress 3: Duda L, van Noort LO, RöselerS, Greitemann BOL, van Harten WH, Klazinga NS. Rehabilitation in Germany and The Netherlands, A comparison of two rehabilitation systems. Roessingh Research and Development, The Netherlands, August 1995, ISBN 90-75452-03-9

Progress 4: Hermens HJ, Nene AV, Zilvold G. Electrophysiological Kinesiology, Proceedings of the 11 th congress of the International Society of Electrophysiology and Kinesiology in Enschede, The Netherlands 1996. Roessingh Research and Development, The Netherlands, October 1996, ISBN 90-75452-04-7

Progress 5: van Harten WH. Bouwen aan een kwaliteitssysteem in de revalidatiezorg. Een poging tot constructieve technology assessment van een kwaliteitssysteem in een gezondheids-zorginstelling. Roessingh Research and Development, The Netherlands, December 1997, ISBN 90-75452-07-1

Progress 6: Baardman G, IJzerman MJ. Design and evaluation of a hybrid orthosis for people with paraplegia. Roessingh Research and Development, The Netherlands, November 1997, ISBN 90-75452-08-X

Progress 7: Hutten MMR. Lumbar Dynamometry: A useful method for assessment of patients with chronic low back pain? Roessingh Research and Development, The Netherlands, November 1999, ISBN 90-75452-13-6

Progress 8: van der Salm A, van Harten WH, Maathuis CGB. Ketenkwaliteit Cerebrale Parese Zorg. Een beschrijving van de cerebrale parese zorg en mogelijke verbeteringen hierin. Roessingh Research and Development, The Netherlands, April 2001, ISBN 90-75452-19-5

Progress 9: Nederhand MJ. Muscle activation patterns in post traumatic neck pain. Roessingh Research and Development, The Netherlands, March 2003, ISBN 90-75452-27-6

Progress 10: Jannink MJA. Usability of custom-made orthopaedic shoes in patients with degenerative disorders of the foot. Roessingh Research and Development, The Netherlands, September 2004, ISBN 90-75452-28-4

Progress 11: Blokhorst MGBG. State-dependent factors and attention in whiplash associated disorder. Roessingh Research and Development, The Netherlands, January 2005, ISBN 90 365-2111-4

Progress 12: Buurke JH. Walking after stroke co-ordination patterns \& functional recovery. Roessingh Research and Development, The Netherlands, February 2005, ISBN 90-365-21408

Progress 13: van der Salm A. Spasticity reduction using electrical stimulation in the lower limb of spinal cord injury patients. Roessingh Research and Development, The Netherlands, October 2005, ISBN 90-365-2253-6

Progress 14: Snoek GJ. Patient preferences for reconstructive interventions of the upper limb in tetraplegia. Roessingh Research and Development, The Netherlands, December 2005, ISBN 90-365-2255-2 
Progress 15: de Kroon JR. Therapeutic electrical stimulation of the upper extremity in stroke. Roessingh Research and Development, The Netherlands, December 2005, ISBN 90-3652269-2

Progress 16: van Dijk H. Motor skill learning, age and augmented feedback. Roessingh Research and Development, The Netherlands, March 2006, ISBN 90-365-2302-9

Progress 17: Mes CAJ. Improving non-optimal results in chronic pain treatment. Roessingh Research and Development, The Netherlands, January 2007, ISBN 90-365-2435-0

Progress 18: Voerman GE. Musculoskeletal neck-shoulder pain: a new ambulant myofeedback intervention approach. Roessingh Research and Development, The Netherlands, March 2007, ISBN 90-365-2460-1

Progress 19: Kallenberg LAC. Multi-channel array EMG in chronic neck-shoulder pain. Roessingh Research and Development, The Netherlands, March 2007, ISBN 90-365-2459-8

Progress 20: Huis in 't Veld MHA. Work-related neck-shoulder pain: The role of cognitivebehavioural factors and remotely supervised treatment. Roessingh Research and Development, The Netherlands, December 2007, ISBN 978-90-365-2584-8

Progress 21: Fleuren JFM. Assessment of Spasticity: From EMG to patients' perception. Roessingh Research and Development, The Netherlands, October 2009, ISBN 978-90-3652869-6

Progress 22: Reenalda J. Dynamic sitting to prevent pressure ulcers in spinal cord injured. Roessingh Research and Development, The Netherlands, October 2009, ISBN 978-90-3652884-9

Progress 23: Prange GB. Rehabilitation Robotics: Stimulating restoration of arm function after stroke. Roessingh Research and Development, The Netherlands, October 2009, ISBN 978-90-365-2901-3

Progress 24: Vos-van der Hulst M. Prognostic factors and underlying mechanisms in chronic low back pain. Roessingh Research and Development, The Netherlands, November 2009, ISBN 978-90-365-2881-8

Progress 25: Kottink-Hutten AIR. Assessment of a two-channel implantable peroneal nerve stimulator post-stroke. Roessingh Research and Development, The Netherlands, February 2010, ISBN: 978-90-365-2959-4

Progress 26: van Weering MGH. Towards a new treatment for chronic low back pain patients. Roessingh Research and Development, The Netherlands, May 2011, ISBN: 978-90365-3180-1

Progress 27: Gulmans J. Crossing Boundaries: Improving Communication in cerebral palsy care. Roessingh Research and Development, The Netherlands, February 2012, ISBN: 978 90-365-3305-8

Progress 28: Molier BI. Influence of augmented feedback on learning upper extremity tasks after stroke. Roessingh Research and Development, The Netherlands, March 2012, ISBN: 978-90-365-3296-9

Progress 29: Dubbeldam R. Towards a better understanding of foot and ankle kinematics in rheumatoid arthritis. Roessingh Research and Development, The Netherlands, October 2012, ISBN: 978-90-365-3407-9

Progress 30: Evering RMH. Ambulatory feedback at daily physical activity patterns. Roessingh Research and Development, The Netherlands, April 2013, ISBN: 978-90-365-3512-0

Progress 31: Malhotra S. Does spasticity interfere with functional recovery after stroke? Roessingh Research and Development, The Netherlands, November 2013, ISBN: 978-90365-3567-0

Progress 32: Tabak M. Telemedicine for patients with COPD. New treatment approaches to improve daily activity behaviour. Roessingh Research and Development, The Netherlands, February 2014, ISBN: 978-94-6108-590-0 
Progress 33: Trompetter HR. ACT with pain. Measurement, efficacy and mechanisms of Acceptance and Commitment Therapy. Roessingh Research and Development, The Netherlands, September 2014, ISBN: 978-90-365-3708-7

Progress 34: op den Akker H. Smart Tailoring of Real-Time Physical Activity Coaching Systems. Roessingh Research and Development, The Netherlands, October 2014, ISBN: 97890-365-3762-9

Progress 35: Jansen-Kosterink SM. The added value of telemedicine for physical rehabilitation. Roessingh Research and Development, The Netherlands, December 2014, ISBN: 978-90-823196-0-6

Progress 36: Velstra IM. Advanced insights in upper limb function of individuals with cervical spinal cord injury. Roessingh Research and Development, The Netherlands, December 2015, ISBN: 978-90-365-3929-6

Progress 37: Kloosterman MGM. Keep on Rolling. Functional evaluation of power-assisted wheelchair use. Roessingh Research and Development, The Netherlands, June 2016, ISBN: 978-90-365-4120-6

Progress 38: Prinsen EC. Adapting to Change. Influence of a microprocessor-controlled prosthetic knee on gait adaptations. Roessingh Research and Development, The Netherlands, December 2016, ISBN: 978-90-365-4206-7

Progress 39: Wolvers MDJ. A coach in your pocket. On chronic cancer-related fatigue and physical behavior. Roessingh Research and Development, The Netherlands, March 2017, ISBN: 978-90-365-4299-9

Progress 40: Cabrita M. Active and pleasant ageing supported by technology. Roessingh Research and Development, The Netherlands, November 2017, ISBN: 978-90-365-4390-3

Progress 41: Haarman JAM, TIBAR - Therapist Inspired Balance Assisting Robot. Roessingh Research and Development, The Netherlands, November 2017, ISBN: 978-90-365-4407-8 

